\title{
Structural Survey on Cohesin and on Viomycin Inhibited 70S Ribosome by Single Particle Electron Microscopy
}

\author{
Dissertation \\ for the award of the degree \\ 'Doctor rerum naturalium' (Dr. rer. nat.) \\ of the Georg-August-Universität Göttingen
}

within the doctoral program 'Biomolecules: Structure - Function - Dynamics' of the Georg-August University School of Science (GAUSS)

submitted by

Michael Hons

from Aachen, Germany

Göttingen, 2015 
Thesis Committee

Prof. Dr. Holger Stark

3D Electron Cryo-Microscopy, Max Planck Institute for Biophysical Chemistry

Prof. Dr. Kai Tittmann

Molecular Enzymology, Albrecht-Haller-Institute

Prof. Dr. Ralf Ficner

Structural Biology, Institute for Microbiology and Genetics

Members of the Examination Board

Reviewer: Prof. Dr. Holger Stark

3D Electron Cryo-Microscopy, Max Planck Institute for Biophysical Chemistry

Second Reviewer: Prof. Dr. Kai Tittmann

Molecular Enzymology, Albrecht-Haller-Institute

Further members of the Examination Board

Prof. Dr. Ralf Ficner (3th member of Thesis Committee)

Structural Biology, Institute for Microbiology and Genetics

Prof. Dr. Detlef Doenecke

Department of Molecular Biology, University Medical Center

Prof. Dr. Marina Rodnina

Physical Biochemistry, Max Planck Institute for Biophysical Chemistry

Prof. Dr. Henning Urlaub

Bioanalytical Massspectrometry, Max Planck Institute for Biophysical Chemistry

Date of the oral examination: 12.05 .2015 


\section{Affidavit}

I hereby declare that this PhD thesis 'Structural Survey on Cohesin and on Viomycin Inhibited 70S Ribosome by Single Particle Electron Microscopy' has been written independently with no other aids or sources than quoted. This thesis (wholly or in part) has not been submitted elsewhere for any academic award or qualification.

Michael Hons 



\section{Contents}

Contents $\quad$ i

List of Figures . . . . . . . . . . . . . . . . . . $\mathrm{v}$

List of Tables ................................. vii

$\begin{array}{ll}\text { Acknowledgements } & \text { ix }\end{array}$

Abstract $\quad$ xi

1 Introduction $\quad 1$

1.1 Molecular Machinery of the Cell . . . . . . . . . . . . . . 1

1.1.1 Life at Cellular Level . . . . . . . . . . . . . . . . . . 1

1.1.2 Physical Laws on Molecular Dimensions . . . . . . . . . . . . . 3

1.1.3 Time and Size Scales . . . . . . . . . . . . . . . . . 3

1.1.4 Principles of Biological Macromolecules . . . . . . . . . . 5

1.1.5 Some Statistics on Macromolecular Complexes . . . . . . . . . . . 6

1.1.6 Impact of Structural Biology . . . . . . . . . . . . . . . 6

1.2 Methods in Structural Biology . . . . . . . . . . . . . . 7

1.2 .1 Overview .......................... 7

1.2.2 Single Particle Electron Cryo-Microscopy . . . . . . . . . . . . 8

1.3 Introduction to Single Particle Electron Cryo-Microscopy . . . . . . . . . 9

1.3.1 Methodical Overview . . . . . . . . . . . . . . 9

1.3.2 Structural Validation . . . . . . . . . . . . . . . . . 12

1.4 Measuring Macromolecular Dynamics . . . . . . . . . . . . . . . 12

1.4.1 Compositional and Conformational Heterogeneity . . . . . . . . . 13

1.4.2 Methods to Investigate Dynamics of Biomolecules . . . . . . . . . 14

1.4.3 Measuring Heterogeneity Using Single Particle EM . . . . . . . . 15

1.5 Cohesin ........................... 15

1.5.1 Replication of Life . . . . . . . . . . . . . . . 15

1.5.2 Identification of Cohesin and Condensin . . . . . . . . . . . 17

1.5.3 Biochemistry of the Cohesin Tetramer . . . . . . . . . . . . 19

1.5.4 Interaction Partners and Regulation of Cohesin . . . . . . . . . . 21

1.5.5 Cohesin Unloading from DNA . . . . . . . . . . . . . 23

1.5.6 Structural Insights into Cohesin . . . . . . . . . . . . . . . . 24

1.6 Prokaryotic Ribosome $(70 \mathrm{~S}) \ldots \ldots \ldots$. . . . . . . . . . . . 28 
1.6.1 Research History of Prokaryotic Ribosome (70S) . . . . . . . . . 28

1.6.2 Structure and Function of the 70 S Ribosome . . . . . . . . . . . . 29

1.6.3 Dynamics of Translocation . . . . . . . . . . . . . . 33

1.6.4 Ribosome-targeting Antibiotics . . . . . . . . . . . 36

1.7 Aim of This Work . . . . . . . . . . . . . . . . 38

2 Materials and Methods $\quad 39$

2.1 Biochemical Approach . . . . . . . . . . . . . . . . 39

2.1.1 Purification of Bonsai Cohesin . . . . . . . . . . . . . . 39

2.1.2 Purification of 70S Ribosome . . . . . . . . . . . . . . 41

2.1.3 Biochemistry and Biophysical Strategies . . . . . . . . . . . 43

2.1.4 Sample Preparation for Electron Microscopy (GraFix) . . . . . . . 44

2.2 Principles of Single Particle Electron Microscopy . . . . . . . . . . . . . 45

2.2.1 Microscope ... . . . . . . . . . . . . . 45

2.2 .2 Electron Source . . . . . . . . . . . . . . . . . . 49

2.2 .3 Electromagnetic Lenses . . . . . . . . . . . . . . . 50

2.2.4 Electron Detection . . . . . . . . . . . . . . . . 50

2.2.5 Microscopic Aberrations . . . . . . . . . . . . . . 51

2.2.6 Real Space and Fourier Space . . . . . . . . . . . . . . 53

2.2.7 Image Formation and Contrast Transfer Function (CTF) . . . . . 54

2.3 Sample Preparation for Single Particle EM . . . . . . . . . . . . . 58

2.3.1 Negative Stain Sample Preparation . . . . . . . . . . . . . . 58

2.3.2 Cryo Sample Preparation . . . . . . . . . . . . . . . . 59

2.4 Image Acquisition . . . . . . . . . . . . . . . . . . . 60

2.5 Image Processing . . . . . . . . . . . . . . . . . . . . 61

2.5.1 2D Level . . . . . . . . . . . . . . . . . . 61

2.5.1.1 Particle Selection . . . . . . . . . . . . . . 62

2.5.1.2 Image Restoration . . . . . . . . . . . . . . . . . . . . . . . . . . . . . . 62

2.5.1.3 Image Averaging . . . . . . . . . . . . . . . . 64

2.5.1.4 Alignment of Images . . . . . . . . . . . . . . . . 65

2.5.1.5 References in Image Alignment . . . . . . . . . . 66

2.5.1.6 Classification of Images . . . . . . . . . . . . . . . . . . . . . . . . . . . . . 67

2.5.1.7 Iterative Process . . . . . . . . . . . . . . 71

2.5.1.8 Projection Matching and Iterative Refinement . . . . . . 72

2.5.1.9 Statistics in Image Processing . . . . . . . . . . . . 74

2.5.2 From 2D to 3D Level . . . . . . . . . . . . . . . 75

2.5.2.1 Angular Reconstitution and 3D Reconstruction . . . . . 75

2.5.2.2 Random Conical Tilt Reconstruction . . . . . . . . . . 77

2.5.2.3 Initial Model Building . . . . . . . . . . . . . . 78 
2.5.2.4 Angular Distribution . . . . . . . . . . . . . 79

2.5.3 Computational Strategies for Sample Heterogeneity . . . . . . . 80

2.5.4 Resolution Assessment . . . . . . . . . . . . . . . . . . . 82

2.5.5 Structural Validation . . . . . . . . . . . . . . . 84

2.6 Summary of Image Processing Procedure in this Work . . . . . . . . . 86

2.7 Equipment and Chemicals . . . . . . . . . . . . . . 88

2.7.1 Special Equipment . . . . . . . . . . . . . . 88

2.7.2 Chemicals . . . . . . . . . . . . . . . . . . . . . 89

2.7 .3 Software . . . . . . . . . . . . . . . . . . . 91

2.7.4 Hardware .......................... 91

3 Results $\quad 93$

3.1 Cohesin . . . . . . . . . . . . . . . . . . . . . 93

3.1.1 Visualisation of Full-length Cohesin . . . . . . . . . . . . . . . 93

3.1 .2 Bonsai Cohesin Tetramer . . . . . . . . . . . . . . . 96

3.1.2.1 Engineering of Bonsai Cohesin Tetramer . . . . . . . . 96

3.1.2.2 Optimised Sample Preparation _. . . . . . . . . . . 97

3.1.2.3 Initial Structural Insights _ . . . . . . . . . . . . . . 97

3.1.3 Pds5 · Bonsai Cohesin Tetramer . . . . . . . . . . . . . . 99

3.1.3.1 Biochemical Integrity of Pds5 Binding to Cohesin . . . . 99

3.1.3.2 Structural Insights into Pds5 Binding . . . . . . . . . . . . 101

3.1.3.3 Compositional and Conformational Heterogeneity . . . . 103

3.1.4 Pds5 · Bonsai Cohesin Trimer . . . . . . . . . . . . . . . . . . . . 108

3.1.4.1 Biochemical Integrity . . . . . . . . . . . . . 108

3.1.4.2 Structural Insights into SA1 Binding . . . . . . . . . . 108

3.1.5 Assignment of Smc1 bonsai Location . . . . . . . . . . . . . . . . 111

3.1.6 Wapl · Pds5 · Bonsai Cohesin Tetramer . . . . . . . . . . . . . . 112

3.1.7 Validation of Cohesin Structures . . . . . . . . . . . . . . . . 113

3.1.7.1 Internal Validation . . . . . . . . . . . . . . . . . . 113

3.1.7.2 "Gold standard" Validation . . . . . . . . . . . . . . . . 114

3.1.7.3 Tilt Validation . . . . . . . . . . . . . . . . . 116

3.2 Influence of Viomycin on 70S Ribosome Dynamics . . . . . . . . . . . . 120

3.2.1 Effects of Viomycin on Ribosomal 30S Body Rotation . . . . . . . 120

3.2.2 Effects of Polyamines on Ribosomal 30S Body Rotation . . . . . . 123

3.2.3 Influence of Viomycin on tRNA Dynamics . . . . . . . . . . . . . 124

4 Discussion $\quad 129$

4.1 Cohesin Model Building and Interpretation . . . . . . . . . . . . . . . . . 129

4.1.1 Structural Conclusions on Bonsai Cohesin . . . . . . . . . . . . . 129 
4.1.2 Validation and Heterogeneity . . . . . . . . . . . . . . . . . . 132

4.1.3 Integration of Available High-Resolution Models . . . . . . . . . . 135

4.1.4 Intermolecular and Intramolecular Crosslinking on Bosai Cohesin 136

4.1.5 Model Building of Pds5 · Full-Length Cohesin . . . . . . . . . . . 139

4.2 Influence of Viomycin and Polyamines on 70S Ribosome Dynamics . . . . 140

5 Summary and Outlook 143

5.1 Topology of an engineered human cohesin complex . . . . . . . . . . 143

5.2 Viomycin and Polyamine Effects on 70S Ribosome Dynamics . . . . . . 145

\section{Appendices}

A Appendix Figures I

B Appendix Text IX

B.1 Crystallography and X-Ray Diffraction . . . . . . . . . . . IX

B.2 Nuclear Magnetic Resonance Spectroscopy . . . . . . . . . . . . . . . X

B.3 Mass Spectrometry . . . . . . . . . . . . . . . . . . . . X XI

B.4 Methods to Adress Biomolecular Dynamics . . . . . . . . . . . . . . . XIII

$\begin{array}{ll}\text { C Abbreviations } & \text { XV }\end{array}$

$\begin{array}{ll}\text { Bibliography } & \text { XXI }\end{array}$

$\begin{array}{ll}\text { Publications } & \text { XLV }\end{array}$

$\begin{array}{ll}\text { Curriculum Vitae } & \text { XLVII }\end{array}$ 


\section{List of Figures}

1.1 Molecular Machinery of the Cell . . . . . . . . . . . . . . . . . 2

1.2 Time and Size Scale of the Molecular Machinery. . . . . . . . . . . . 4

1.3 Overview of the Single Particle EM Workflow. . . . . . . . . . . . . . . 9

1.4 Compositional and Conformational Heterogeneity . . . . . . . . . . . . 14

1.5 Biochemistry of Cohesin . . . . . . . . . . . . . . . . . 21

1.6 Relevant Structural Models of Cohesin Subunits . . . . . . . . . . . . 25

1.7 Overview of Prokaryotic Translation . . . . . . . . . . . . . . . 31

1.8 Viomycin Effect on Translocation . . . . . . . . . . . . . . . . 34

2.170 S Ribosome Dissociation Monitored by Light Scattering . . . . . . . . 43

2.2 Sample Stabilisation with GraFix . . . . . . . . . . . . . . 45

2.3 Composition of a TEM. . . . . . . . . . . . . . . . . . 48

2.4 Concepts of Image Formation . . . . . . . . . . . . . . . 54

2.5 Effect of Defocusing on the Phase Contrast Transfer Function (PCTF). . 58

2.6 Application of Fourier Filters . . . . . . . . . . . . . 64

2.7 Alignment and Classification . . . . . . . . . . . . . . 68

2.8 Iterative Moduls during Image Processing. . . . . . . . . . . . . . . 73

2.9 Model Bias can be induced by a Wrong Reference Models. . . . . . . . . 80

3.1 2D Representation of Full-lengh Cohesin . . . . . . . . . . . . . . 95

3.2 Engineering of Bonsai Cohesin Tetramer . . . . . . . . . . . . . . 96

3.3 Optimised Sample Preparation . . . . . . . . . . . . . . . . . . 98

3.4 Initial Structural Insights into Bonsai Cohesin Tetramer . . . . . . . . . 100

3.5 Biochemical Integrity of Pds5 Binding to Cohesin . . . . . . . . . . . . . 102

3.6 First Structural Insights into Pds5 • Bonsai Cohesin Tetramer . . . . . . 104

3.7 Improved 3D Model of Bonsai Cohesin Tetramer . . . . . . . . . . . . . . 105

3.8 Heterogeneity within Pds5 · Bonsai Cohesin Tetramer . . . . . . . . . . 107

3.9 Biochemical Integrity of Pds5 • Bonsai Cohesin Trimer . . . . . . . . . . 109

3.10 Structural Insights into Pds5 · Bonsai Cohesin Trimer . . . . . . . . . 110

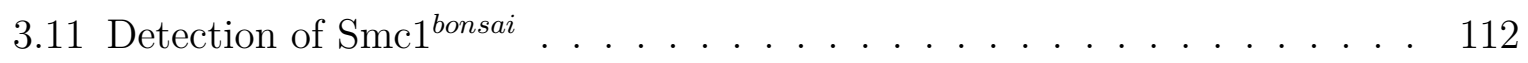

3.12 Density Recovery from $\Delta$ Reference Models for Density Validation . . . . 115

3.13 "Gold standard" Validation . . . . . . . . . . . . . . . . . . 117

3.14 Tilt Validation ． . . . . . . . . . . . . . . . . . . . . . . . . . . . . . . . 119

3.15 Viomycin affects the 30S Body Rotation . . . . . . . . . . . . . . 122 
3.16 High Polyamine Concentration influence the 30S Body Rotation . . . . . 124

3.17 Influence of Viomycin on tRNA Dynamics . . . . . . . . . . . . . 126

4.1 Side by Side Comparison of distinct Bonsai Cohesin Structures . . . . . . 133

4.2 Integration of Structural Data . . . . . . . . . . . . . . . 137

4.3 Cross-links within Full-Length Cohesin and Bonsai Cohesin . . . . . . . . 138

A.1 Number of Released Structures by Different Structural Methods . . . . . I I

A.2 ATPase Activity of Bonsai Smc3 . . . . . . . . . . . . . . . II

A.3 Substoichiometric Wapl Contamination . . . . . . . . . . . . . . III

A.4 Comparison of the Extracted Pds5 Density and a Homology Model . . . IV

A.5 Sample Quality of Wapl · Pds5 · Bonsai Cohesin Tetramer . . . . . . . . V V

A.6 Initial 3D models of Wapl · Pds5 · Bonsai Cohesin Tetramer . . . . . . . VI

A.7 Viomycin Effect on 30S Body Rotation . . . . . . . . . . . . . VII 


\section{List of Tables}

1.1 Comparison between Different Structure Determining Methods . . . . . . 8

1.2 Composition and Interaction Partners of Human Cohesin Complex . . . 19

1.3 Properties of the Prokaryotic 70S Ribosome . . . . . . . . . . . . . 30

2.1 Preparation Condition of Ternary Complex (TC) . . . . . . . . . . . . 42

2.2 Preparation of Initiation Complex (IC) . . . . . . . . . . . . . . 42

2.3 Final Mixture of Initiation Complex (IC) and Ternary Complex (TC) . . 42

2.4 Image acquisition at Philips CM200 . . . . . . . . . . . . . . 61

2.5 Image acquisition at FEI Titan Krios . . . . . . . . . . . . . . . . 61

2.6 Special Equipment . . . . . . . . . . . . . . . . . . 88

2.7 Used Chemicals . . . . . . . . . . . . . . . . . . . . . . . . . . . . 89

2.8 Used Laboratory Materials . . . . . . . . . . . . . . . . . . . . . . 90

2.9 Used Software . . . . . . . . . . . . . . . . . . . . . . . . . . 91

2.10 Used Hardware (local device) . . . . . . . . . . . . . . . . . . . . 91

3.1 Cohesin nomenclature . . . . . . . . . . . . . . . . . . . . 94 


\section{Acknowledgements}

Four years ago, I joined the scientific field of single particle electron cryo-microscopy. First, I would like to thank Prof. Dr. Holger Stark for giving me the opportunity to contribute to this fascinating world of structural biology. This work would not have been possible without the outstanding technical equipment and the great knowledge within the lab of Prof. Dr. Holger Stark. In his lab, I was able to greatly increase my scientific knowledge in practice and theory and worked on several different and exciting scientific questions. Moreover, I am thankful for the opportunity to join many international scientific meetings and conferences.

This work would not have been possible without the great collaboration with the mitosis and chromosome biology lab of Prof. Dr. Jan-Michael Peters at IMP Vienna. I had the opportunity to take part in very fruitful meetings at IMP Vienna. My special thanks I address to Dr. Pim Huis in 't Veld. He enabled the collaboration on cohesin, and his prior biochemical work on the reconstitution of human cohesin complex is the basis for a great part of the present work. Pim introduced me to the thrilling cohesin world and enabled me to benefit from his expertise on cohesin. Our collaboration developed into a scientific friendship and I enjoyed the enthusiasm for the project and the spare time activities, like the dragon boat race on the Danube.

The ribosome-related part of this work benefited greatly from the huge expertise of Dr. Niels Fischer. I am very grateful that Dr. Niels Fischer shared his practical knowledge of electron microscope usage, image processing and ribosomes. For this reason, the ribosomal project would not have been possible without his commitment. In this context, I like to thank the lab of Prof. Dr. Marina Rodnina and especially Dr. Frank Peske for providing $70 \mathrm{~S}$ ribosome samples, whenever I needed fresh samples and for being so helpful whenever I had questions.

I like to thank Dr. Wen-Ti Liu for her friendship and support when I had questions related to microscopy, image processing and data illustration. Her patience and her willingness to help others are outstanding. Her friendship supported me throughout this thesis.

During this work, I had the chance to share my scientific knowledge with Jan-Michael Kaesler. He joined the cohesin project as Master student and training him was a great pleasure. By training someone else I deepened my knowledge and could scrutinise my own thinking. I wish him good luck for his private and scientific future. 
Many more colleagues helped this work to be successful. I am thankful to the IT specialists in our lab. In this context I want to thank equally Georg Bunzel, Dr. Boris Busche, Dr. Jan Kirves and Dr. Mario Lüttich. I am thankful to Dr. Stephanie Schell and enjoyed the pleasant atmosphere with her. I am personally grateful to Dr. Andrius Krasauskas and Frank Würriehausen. Moreover, I am thankful to Dr. Ashwin Chari, Dr. David Haselbach, Dr. Dietmar Riedel, Jan Erik Schliep and Jil Schrader. Thanks to all other people in our growing lab, especially the new members, for the on-going positive lab atmosphere. For her advice and patience related to bureaucratic work, I like to thank our secretary Juliane Moses.

Not least, I would like to thank my thesis committee members Prof. Dr. Kai Tittmann and Prof. Dr. Ralf Ficner for their advices and help and the friendly atmosphere during the thesis committee meetings. The concept of thesis committees helped to guideline through many years of hard work and created intermediate goals to share and improve the progress of this work. Equally, I am grateful to my extended thesis committee members: Prof. Dr. Detlef Doenecke, Prof. Dr. Marina Rodnina and Prof. Dr. Henning Urlaub.

Finally, I want to thank my friends inside and outside of Göttingen. Their friendship encouraged me significantly since I came to Göttingen in 2011. I thank my family for accompanying me throughout my life. I cannot thank enough Tristan for his warmth and company. 


\section{Abstract}

Life can be understood as the systematic collaborative action of several entities on molecular level reducing locally the entropy at the cost of the exterior environment. These entities are in great part macromolecular complexes composed of different proteins and ribonucleic acids. In a bottom-up approach, structural studies on macromolecular complexes can help to understand the principles of life.

In this work, the structures of two very different macromolecular complexes, the human cohesin and the E. coli 70S ribosome, are studied by single particle electron microscopy. Structural studies on the $70 \mathrm{~S}$ ribosome are well established, and very sophisticated questions regarding small binding partners or specific dynamics can be addressed today. In comparison, structural studies on cohesin are rather young and started less than 20 years ago. Only the structural definition of individual domains or subunits was possible so far. A complete model of cohesin has not been defined prior to the present work.

Single particle electron microscopy has proved to be superior to other structure defining methods in terms of studying large macromolecular assemblies (>200 kDa). Moreover, it is competent in resolving dynamics of these entities to a higher extent than any other method. In this work, it was aimed to study the influence of viomycin on the dynamics of the $70 \mathrm{~S}$ ribosome. Moreover, it was aimed to generate the first structural model of cohesin. Single particle electron microscopy was the method of choice to study both, the $70 \mathrm{~S}$ ribosome and cohesin.

Cohesin, a key regulator of sister chromatid cohesion, is mandatory for maintaining the topological arrangement of chromatin. Especially mitosis and meiosis require the correct and equal allocation of sister chromatids into daughter cells. An engineered cohesin version that largely lacks the long and flexible coiled-coil domains of Smc1 and Smc3 was shown to be an active ATPase and to interact with regulatory cohesin subunits (Pds5, Wapl). Due to the lower flexibility, this "bonsai cohesin" is more suitable to be studied by electron microscopy than its full-length counterpart.

In this study, the low-resolution structure of a bonsai cohesin tetramer (bonsai Smc1/3, Scc1, SA1), Pds5 - bonsai cohesin tetramer (bonsai Smc1/3, Scc1, SA1, Pds5) and Pds5 - bonsai cohesin trimer (bonsai Smc1/3, Scc1, Pds5) have been solved ab initio. By comparison of these three independently obtained EM models, specific densities, which were not present in all three models, have been attributed to Pds5 and SA1. Pds5 was shown to be located at the bottom of the Smc1/3 nucleotide binding domains (NBDs), 
and it seems to bend around both Smc NBDs. The size of the obtained Pds5 density corresponds to the expected size of the protein and shows similarity to a generated homology model. For Pds5, no crystal structure is available, and the binding site of Pds5 had not been known prior to this work. Computational sorting allowed to resolve a compositional heterogeneity of Pds5 occupancy within the sample of Pds5 - bonsai cohesin tetramer. SA1 appears to be located at the back of the junction of the Smc1/3 NBDs and the Smc1/3 hinge domain. A 2xRFP tag was introduced to distinguish the Smc1 NBD from Smc3 NBD. An additional density is proposed to correspond to this tag. Accordingly, the correct attribution of Smc1 NBD and Smc3 NBD seems to be likely. The fitting of the available crystal structures is in accordance to the topology, defined solely by single particle electron microscopy without any remaining undefined density segments. Accordingly, a complete topology map of the crystal structures (Smc1/3 hinge domain, Scc1(C)-Smc1 NBD, Scc1(N)-Smc3 NBD and SA1-Scc1(middle) within the EM model of bonsai cohesin was possible. The most detailed model (bonsai cohesin tetramer) reaches a resolution of roughly $27 \AA$. It appears that the low resolution is related to cohesin-specific heterogeneity due to the flexibility of the shortened coiled-coils. Based on this work, future structural studies may be enabled to obtain higher resolved 3D models of cohesin.

In the second part of this study, the influence of viomycin on the $70 \mathrm{~S}$ ribosome dynamics was studied. Viomycin is a translocation-inhibiting antibiotic that was shown to stabilise A-site tRNA and to be located between h44 (30S subunit) and H69 (50S subunit). The focus of this work is to understand the inhibitory mechanism with respect to a change in the dynamical landscape. Prior to this work, the effect of viomycin on these dynamics had been discussed controversially. Specifically, the effect on the $30 \mathrm{~S}$ body rotation and the tRNA dynamics had been unclear.

In the present work, it has been shown that viomycin induces higher $30 \mathrm{~S}$ body rotation states. High concentrations of polyamines (spermidine and putrescine) can promote a similar but smaller effect on 30S body rotation. Regarding the 30S body rotation, the effect of one viomycin molecule can be mimicked by the collaborative action of several polyamine molecules. As high degrees of 30S body rotation are loosely coupled to more frequent tRNA hybrid states, it was examined if hybrid states are possibly induced by viomycin. Preliminary results suggest favoured hybrid states when viomycin is present. Further results are awaited to draw distinct conclusions. The effect of viomycin on $70 \mathrm{~S}$ ribosome dynamics can be understood as a proof of principle that single particle electron cryo-microscopy is an appropriate way to address the inhibitory effect of a small molecule with respect to changing macromolecular complex dynamics.

Keywords: single particle electron microscopy, image processing, cohesin, Pds5, 70S ribosome, viomycin, structural dynamics of protein complexes 


\section{Chapter 1}

\section{Introduction}

Life seems to be orderly and lawful behaviour of matter, not based exclusively on its tendency to go over from order to disorder, but based partly on existing order that is kept up.

- Erwin Schrödinger

\subsection{Molecular Machinery of the Cell}

\subsubsection{Life at Cellular Level}

Although in some aspects we are able to obtain an atomistic and picosecond understanding of the molecular processes in cells, for many questions this information and the overall picture of the vast amount of parallel processes related to life stays out of reach. Life must be understood as a sophisticatedly organised, compartmentalised and energy-driven system, where the dynamic interplay of the cell's interior replicates itself in a programmedlike fashion (Daniel E. Koshland, 2008). The ingredients of a cell are mainly proteins, RNA, polysaccharides, lipids, DNA and various small molecules in water. Especially proteins and RNA are the modular building blocks for higher order structures, so-called macromolecular complexes, which are the principal performers in the cellular systems. These macromolecular complexes interact in a highly cooperative and regulated way, meaning that physical interactions between those complexes are changed all the time, complexes are rapidly assembled and disassembled according to their underlying task. These interactions and regulations cannot be described in the same way as processes of macroscopic engines. Still, the term "molecular machine" is appropriate, as it helps to understand the energy-driven processes within the cell in a directional, causal and deterministic way (Goodsell, 2009, Chapter 2) (Figure 1.1). These molecular machines run all the processes which are essential for the cell's viability. In the following section, the physical laws which are relevant in molecular dimension will be described. 
a

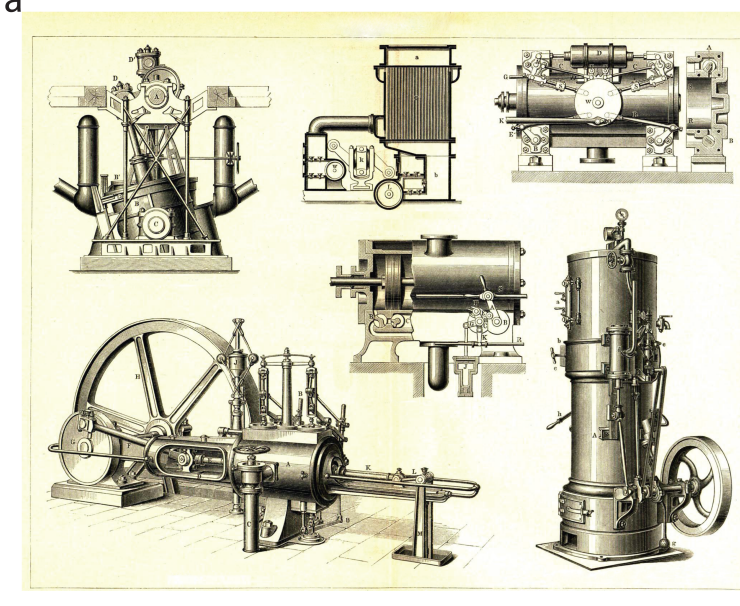

C

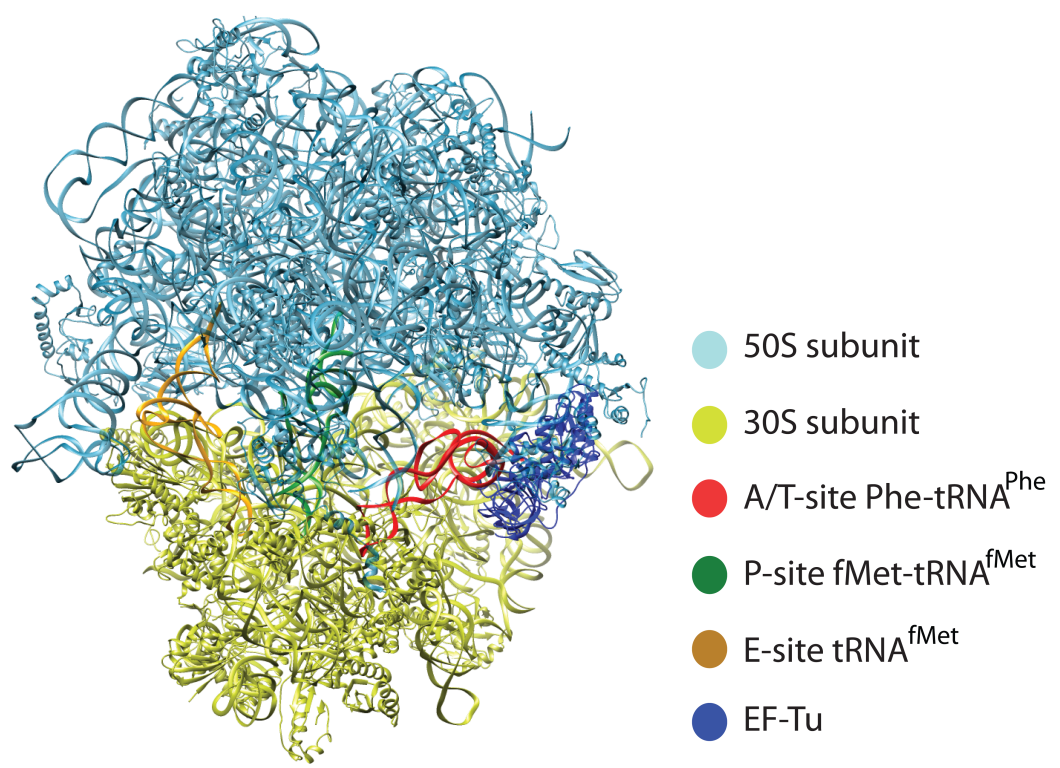

b

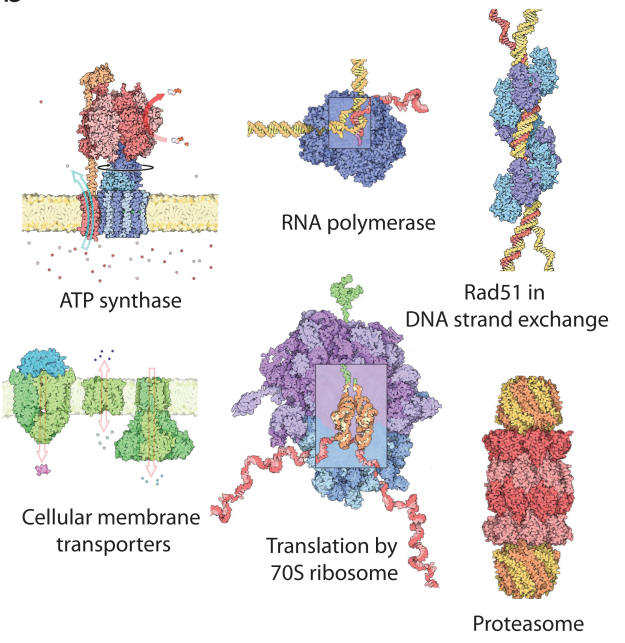

Figure 1.1: Molecular Machinery of the Cell. a: Illustrated are different kind of steam-engines as examples of different man-made machines. Obvious is the structure-function relationship in those machines. b: A similar structure-function relationship is apparent in some exemplary illustrated molecular machines. Although on nanoscale the contributions of different physical laws on the functionality of those machines is different, the analogy to man-made machines is useful to understand the principles on molecular scale. The relative scale of the depicted molecular machine is realistic. Schemes in a and b are modified and illustrated with permission from Meyer (1885) and Goodsell (2009). c: The $<3 \AA$ resolution structure of $E$. coli ribosome-EF-Tu complex solved by $\mathrm{C}_{S}$-corrected cryo EM is an example of a near-atomic resolution structure of a molecular machine (Fischer et al., 2015). The combined action of 50S subunit, 30S subunit, mRNA, tRNAs and additional factors allows the ribosome to translate the genetic information stored in mRNA into functional proteins. 


\subsubsection{Physical Laws on Molecular Dimensions}

Although the physical laws in macroscopic and nanoscopic worlds are principally the same, meaning both can be approximately described by classical physics, the contribution of different forces changes remarkably on the nanoscale. In the description of diffusion, gravity and inertia can be neglected, whereas the viscosity of a solution becomes a dominating force $^{1}$. Compared to macroscopic machines, which are mainly influenced by gravity, inertia and friction, molecular machines are limited by the viscosity of the cellular solution, while the former play a minor role (Goodsell, 2009, Fig. 1.4) (Serdyuk et al., 2007, D1,D2,D3). Furthermore, Brownian motion plays an important role for molecular machines, explaining their internal flexibility and trembling movement within solution ${ }^{2}$.

In addition, molecules are densely packed within the cell, a condition named "molecular crowding", meaning that typically $25-35 \%$ of space is filled, reducing the mean free path for Brownian motion and diffusion dramatically (Ellis, 2001; Zimmerman and Minton, 1993). Although the average and undirected velocity of a small molecule is around 5 $\mathrm{m} / \mathrm{s}$ (Goodsell, 2009, The Molecular World), molecular crowding will reduce the effective velocity of a molecule to cover a certain distance 100-fold (Ellis, 2001; Chebotareva et al., 2004). Anyhow, this is fast enough that almost every molecule in the cytoplasm of a bacterial cell will meet each other molecule within seconds. This is the prerequisite that cell signalling and all molecular processes are functional. On the other hand, macromolecular complex formation may increase by two to three orders of magnitude due to the gain of effective concentration and chemical activity ${ }^{3}$. This means that once two molecules bind to each other in a molecular crowded environment, they are less likely removed from each other than in free solution. Obviously, these considerations are important for the rate and equilibrium of macromolecular complex formation (Philipse, 2011). Of further interest are the corresponding time and length scales, which will be appraised in the following section.

\subsubsection{Time and Size Scales}

Molecular processes are happening at very fast time scales as shown for some examples (Figure 1.2c). Ultra-fast reactions like the electronic reorganisation of retinal upon electro-

\footnotetext{
${ }^{1}$ A strong influence of viscosity is defined by a low Reynolds number. The force can be described by the Navier-Stokes equation.

${ }^{2}$ To describe Brownian motion, the Wiener process is used. The positive average distance that a particle covers in time is proportional to the temperature and inversely proportional to the particle's radius (Philipse, 2011).

${ }^{3}$ Actually, it has to be distinguished between diffusion-limited or transition-state-limited processes, meaning that the overall reaction is transition-state-limited at low concentration and diffusion-limited at high concentration.
} 
magnetic activation happen in the femtosecond (fs) range (Serdyuk et al., 2007, Fig. 3, Fig. 4)(Alberts et al., 2008). In comparison, thermal fluctuations are in the picosecond (ps) range. DNA unfolding happens in nanoseconds (ns), and finally, enzymes catalyse reactions within microseconds (ms). For example, prokaryotic RNA polymerase II transcribes mRNA with a speed of 50 bases per second and a prokaryotic ribosome has an average speed of nearly 60 bases per second (s), generating a chain of 20 amino acids (aa) per second. These examples are summarised according to Alberts et al. (2008), Serdyuk et al. (2007) and Goodsell (2009).

a

b

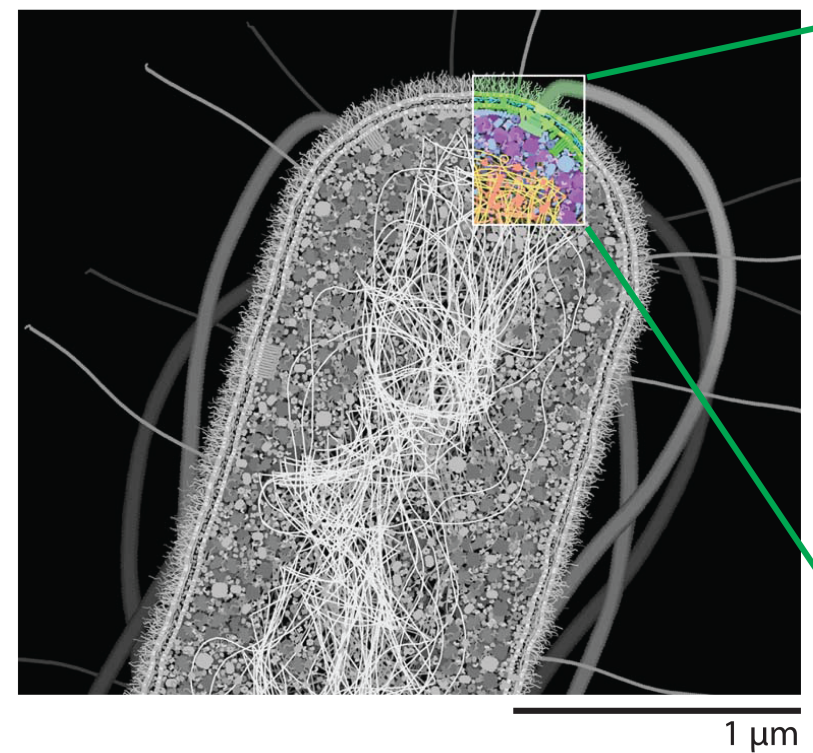

C
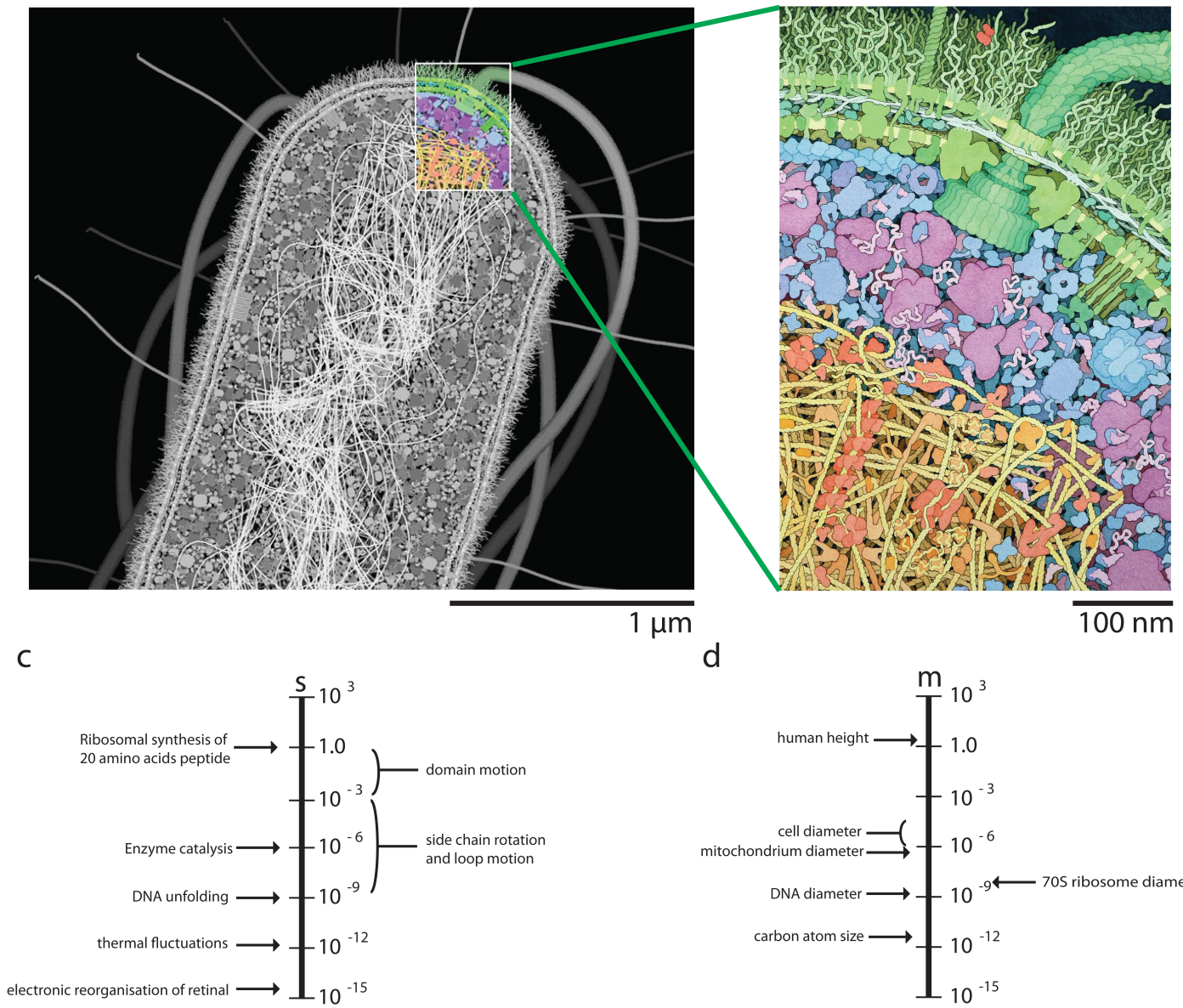

d

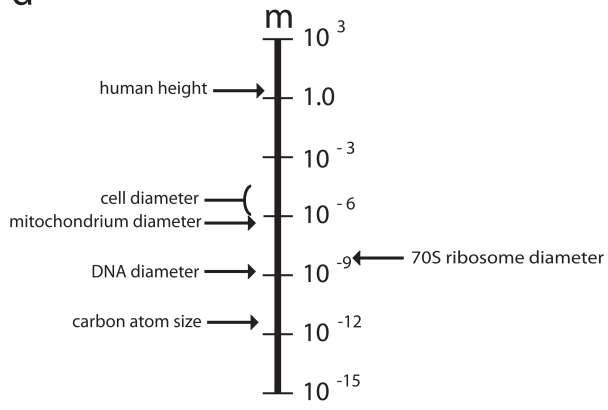

Figure 1.2: Time and Size Scale of the Molecular Machinery. a: The overall architecture of a living cell in case of $E$. coli shows the dense packing of functional components within the cell surrounded by a double-layered cell wall. The dense packing is referred to as molecular crowding. b: In a close-up, the higher order organisation becomes obvious. In yellow and red, nucleoid components are shown. The transcription-, translation- and protein-folding-related machinery is depicted in the intermediate layer (red, purple and blue), and in green, cell wall components are depicted. Schemes in $\mathbf{a}$ and $\mathbf{b}$ are modified and illustrated with permission from Goodsell (2009). c: The approximate time scale of exemplary cellular processes is shown in seconds. The illustration is based on Serdyuk et al. (2007, Fig. 4) and Alberts et al. (2008) d: The cellular organisation is illustrated from atomic up to macroscopic scale. The $70 \mathrm{~S}$ ribosome defines roughly the upper size limit of molecular machines (Alberts et al., 2008; Goodsell, 2009). The illustration is based on Serdyuk et al. (2007, Fig. 3).

Similarly, it is reasonable to evaluate the size scale of the molecular machinery exemplary for some biological objects (Figure 1.2d). The size of a cell is in the order of 1-100 $\mu \mathrm{m}$, 
the mitochondrion has a diameter of 0.5 to $1 \mu \mathrm{m}$, the T4 bacteriophage has a size of approximately $200 \mathrm{~nm}$, whereas one of the smallest viruses, Porcine circovirus, has an icosahedral capsid of $17 \mathrm{~nm}$ in diameter. This virus is already in the range of huge macromolecular machines, for instance the ribosome with a diameter of $25 \mathrm{~nm}$. DNA has a diameter of $2 \mathrm{~nm}$, and the size of a single carbon atom is $67 \mathrm{pm}$. These examples are summarised according to Serdyuk et al. (2007), Alberts et al. (2008) and Goodsell (2009).

\subsubsection{Principles of Biological Macromolecules}

In contrast to their macroscopic counterpart, molecular machines are highly flexible units. At room temperature, the available thermal energy for Brownian motion exceeds the available chemical energy by several orders of magnitude. The question is how directional movement can be obtained. Any engine, driven by constant temperature, would be a perpetual motion machine of the second kind. However, this is not possible according to the second law of thermodynamics, as the entropy of isolated systems can never be decreased. In this context, it was shown that the idea of a molecular ratch using Brownian motion is conceptionally impossible (Feynman, 1964). However, one can derive a directional motion from Brownian motion using an energy field on a molecular ratch while the energy field is alternately turned on and off, allowing on average particles to move slowly, compared to the undirected movement, in one direction. This is the principle used by biological machines. One example is the ribosome, where EF-G drives tRNA translocation directionality by GTP cleavage (Cunha et al., 2013). Another example is the coupling of a proton gradient to the ATP concentration by ATP synthase (Boyer, 1997). In a bidirectional process, the machine works either as ATP-consuming proton pump or as a proton-pump-driven ATP synthase. The energy field is generated by ATP or proton concentration gradients.

In many textbooks, the wrong impression is given that enzymes act as single units and are regulated by short-time ligand binding events. For several aspects of life, it seems very advantageous to build up protein complexes instead of functioning on the basis of single proteins, some of them being named in the following passage (Alberts et al., 2008):

1. Increase of network complexity:

Each binding event can be understood as a switch, changing the state of the complex in an on/off fashion independently for each possible interaction partner. Thereby, complex and highly regulated networks are achieved.

2. Recycling:

Complexes can be disassembled and newly assembled if only one subunit is inop- 
erative. Recycling of complexes is more efficient than the use of equivalent single proteins, which need to be newly synthesised in case of malfunction.

Protein interaction is based on complementarity of the surface of interacting proteins, by "lock-and-key", "induced-fit" and "conformational selection" (Boehr et al., 2009; Csermely et al., 2010). The complementarity is defined by topological fitting of the involved atoms, electrostatic interactions (ionic or van der Waals' interactions) and hydrogen bonds. The affinities between subunits are defined by dissociation constants $\left(\mathrm{K}_{\mathrm{d}}\right)$. A linear correlation between $\log \left(\mathrm{K}_{\mathrm{d}}\right)$ and the buried surface area (BSA) of two interacting proteins can be defined. However, the affinities are relatively low compared to small molecule interactions. A typical BSA of $2000 \AA^{2}$ between two proteins relates to a $K_{d}$ of $100 \mathrm{nM}$, which is relatively low compared to the $K_{d}$ values of small molecules, which are in the low $\mathrm{pM}$ range (Chen et al., 2013). In general, calculated $K_{d}$ values need to be corrected for the effect of molecular crowding (Philipse, 2011; Zimmerman and Minton, 1993).

\subsubsection{Some Statistics on Macromolecular Complexes}

Recently, systems biology and proteomics helped to better understand the proteomic network, resulting in improved predictions of potential protein complexes. The human genome project has revealed that the complexity compared to yeast is not reflected solely by the number of genes but by the number of interactions, defined by the so called "interactome" ${ }^{4}$ (Sharon, 2010). A large-scale analysis of the yeast proteome identified 232 protein complexes from 1739 processed genes and resulted in an average complex size of $\sim 3$ protein subunits, while $51 \%$ of the identified protein complexes had 1-5 subunits (Gavin et al., 2002). According to a median protein size of 280 aa, this results in an median complex size of up to $\sim 154 \mathrm{kDa}$. In the human proteome, 5584 distinct human proteins were identified, predicting 622 discrete putative complexes (with 2634 distinct proteins) (Havugimana et al., 2012). Here, a median complex size of 4 subunits per complex can be defined. According to the median length of human proteins, this relates to an average protein complex size of $\sim 165 \mathrm{kDa}$. Solving the structural arrangement of these macromolecular complexes allows to deduce more about their biochemical functionality.

\subsubsection{Impact of Structural Biology}

Up to now, there are more than 100,000 macromolecular structures of proteins, RNAs and complexes of either protein, RNA, or both released in the corresponding databases (PDB:01.2015). R. Feynman claimed that it is "very easy to answer many [...] fundamental

\footnotetext{
${ }^{4}$ The quotient of genome size is $(\Sigma$ yeast genome $) /(\Sigma$ human genome $)=0.16$, whereas the quotient of the interactome size is $(\Sigma$ yeast interactome $) /(\Sigma$ human interactome $)=0.05$.
} 
biological questions, by just looking at the thing" (Quirk, 2006). However, visual observations are technically challenging due to very small size scales, relatively fast time scales and the imperfections of the optical observation system. Correspondingly, structural biology is a dramatically growing scientific field using several techniques to solve the structures of a biological entity. These techniques are continuously being improved and new techniques are developed. This is particularly true for single particle electron cryo-microscopy (cryo EM or single particle $\mathrm{EM}^{5}$ ), which is, compared to X-ray diffraction and Nuclear Magnetic Resonance (NMR), a relatively young method. The following statistics will point out the growing role of cryo EM. $79 \%$ of all structures solved are below $100 \mathrm{kDa}$ in size and $96 \%$ are below $300 \mathrm{kDa}$ (PDB Statistics, 2015). According to Section 1.1.5, the average size of a molecular complex is $\sim 150 \mathrm{kDa}$. Thus, there is a strong demand for techniques which are capable to analyse those objects. NMR is not capable to solve structures above $\sim 100 \mathrm{kDa}$, and for X-ray diffraction it is still very hard, as it is difficult to grow crystals of bigger and potentially more dynamic structures. There are only few exceptions of very stable big molecular machines, which allow crystal growth (Mueller et al., 2007; Ban et al., 2000; Schluenzen et al., 2000). In contrary, there is no upper but a lower size limit for cryo EM $(>\sim 200 \mathrm{kDa})$. Thus, there is a great potential to determine the many structures by cryo EM which cannot be analysed by other methods. To emphasise this even more, currently, PDB statistics show an roughly exponential growth of structures being determined by cryo EM, whereas only a linear growth of structures solved by NMR and X-ray diffraction can be determined (Figure:A.1).

\subsection{Methods in Structural Biology}

\subsubsection{Overview}

In the following section, different structural methods will be highlighted and the specific advantages and limitations of each technique will be summarised. Depending on the specific structural question varying structural methods or combinations of those may be necessary. While NMR is the method of choice for peptides and small proteins and can give information about dynamics of a sample in solution, X-ray diffraction is used for bigger entities (mainly $<\sim 200 \mathrm{kDa}$ ). The corresponding proteins need to be crystallised, which can be a challenging obstacle. Mass spectrometry (MS) has become increasingly important in the last two decades, especially since the development of electron spray ionisation (ESI), allowing proteins to stay intact while being ionised. As it provides stoichiometric and topological information on macromolecular complexes with sizes ranging up to approximately 2 MDa,

\footnotetext{
${ }^{5}$ The term "cryo EM" is used only when single particle EM of vitrified sample is meant. When referring to single particle EM (including negative stain data) the term "single particle EM" is used.
} 
Table 1.1: Comparison between Different Structure Determining Methods

\begin{tabular}{l|l|l|l|l} 
parameter & NMR & X-ray crystallography & mass spectrometry & cryo EM \\
\hline sample amount & $>5 \mathrm{mg}$ & $20-100 \mathrm{mg}$ & $1 \mathrm{ng}-1 \mu \mathrm{g}$ & $\mathrm{a}$ few $\mu \mathrm{g}$ \\
accessible mol. weight & $\begin{array}{l}\text { mainly }<\sim 100 \\
\mathrm{kDa}\end{array}$ & mainly $<\sim 200 \mathrm{kDa}$ & $\mathrm{mainly}>\sim 2 \mathrm{MDa}$ & $\mathrm{mainly}>200 \mathrm{kDa}$ \\
achievable resolution & not applicable & $1-5 \AA$ & only topology \\
accessible dynamics & $\begin{array}{l}\text { side chain rotation } \\
\text { global folding }\end{array}$ & rarely possible & subunit rearrangment & $\begin{array}{l}\text { small and big changes } \\
\text { in subunit tumbling and } \\
\text { movement }\end{array}$ \\
\hline
\end{tabular}

it has become extremely helpful in structural biology (Sharon, 2010; Walzthoeni et al., 2013; Herzog et al., 2012). Finally, single particle EM, the only structural single particle method, is developing very fast, reaching, for certain macromolecular complexes, resolution levels below $\sim 3 \AA$ (Fischer et al., 2015) and decreasing the lower size limit to $\sim 200 \mathrm{kDa}$ (Monecke et al., 2012). Additionally, the method is capable to study second to minute dynamics. Table 1.1 gives an overview about the capabilities of the mentioned techniques. In the appendix of the present work, more information related to crystallography and X-ray diffraction (Appendix section B.1), NMR (Appendix section B.2) and Mass spectrometry (Appendix section B.3) is summarised.

\subsubsection{Single Particle Electron Cryo-Microscopy}

Cryo EM dates back to 1984, when the successful vitrification of an adenovirus suspension was first shown (Adrian et al., 1984). This breakthrough opened the door for high resolution cryo $\mathrm{EM}^{6}$. Since then, cryo EM has made great improvements in sample preparation, microscopy and data processing, generating structures at near-atomic resolution $(<3.0$ A) (Fischer et al., 2015). Not only the maximal attainable resolution improved, also the minimal size of examinable structures has shifted to a lower size (Monecke et al., 2012). Especially the use of new direct electron detector devices with counting mode based on CMOS technology improved imaging quality drastically (Faruqi and Henderson, 2007). The increasing computational power, together with more sophisticated image processing algorithms, allows sorting of data for different conformational states, generating high resolution data on the conformational landscape of some molecular machines. In summary, the gap in resolution and size between different structural methods is closing, with an outstanding importance of cryo EM for structures which were not addressable before by structural means (Kühlbrandt, 2014). Details about sample preparation, image acquisition and data processing can be found in Chapter 2. A methodological overview is given in Figure 1.3.

\footnotetext{
${ }^{6}$ Already in 1981, J. Dubochet validated that vitrification of pure water is possible (Dubochet and McDowall, 1981).
} 


\subsection{Introduction to Single Particle Electron Cryo- Microscopy}

At first, a methodological overview of single particle electron cryo-microscopy will be given. Especially, the general work-flow of sample preparation and image processing will be outlined (Figure 1.3). A project starts with a molecular machine of interest, concerning a certain biological question. Successful purification of native complexes or purification from a host organism followed by in vitro reconstitution can be tedious and long-lasting, especially for structural biology purpose. This is due to the requirement of high sample quality and usually, a sample must be optimised before it can be used for any structural method. For EM, a pure and stable sample is needed. In the following section, sample preparation, microscopy and image processing are briefly outlined (Orlova and Saibil, 2011; Elmlund and Elmlund, 2015). More details are given in Section 2.2.

\subsubsection{Methodical Overview}

In Figure 1.3a, the overall work-flow of single particle EM is shown. A purified macromolecular complex may still be optimised for single particle EM. The homogeneity and stability of a macromolecular complex can be optimised by gradient fixation (GraFix) (Kastner et al., 2008). In a gradient solution (e.g. sucrose), complexes sediment according to their mass. An additional gradient of a cross-linker (e.g glutaraldehyde (GA)) can stabilise the

Figure 1.3 (following page): Overview of the Single Particle EM Workflow. a: A pure and homogeneous protein complex needs to be purified and prepared for EM, either by negative stain preparation or vitrification. After satisfying conditions are defined (sample and preparation quality), images are recorded. The obtained micrographs contain several noisy particle images. Particle images are extracted and processed on 2D level by summing similar particle images to increase the contrast. The obtained images can be used to generate an initial 3D model. Several iterations of 2D and 3D processing yield a final 3D model, which needs to be validated and interpreted. $\mathbf{b}$ : The details of 2D and 3D image processing are depicted from (1) to (5). An area of a micrograph is shown (1), with particles to be selected. Manual, semi-manual and automated particle selection tools can be applied. The selected particle images are stored in an image stack. Preprocessing (2) includes CTF correction, where negative spatial frequencies are flipped, and downsampling to reduce the data size and to increase the contrast. Subsequent filtering allows to reduce unwanted gradients (low spatial frequency domain) and random noise (high spatial frequency domain). In 2D processing (3), images are aligned to bring them into similar orientation and grouped to sum up equivalent and aligned projection (the so-called classification). In the summed images (so-called class averages) the noise is reduced and the particle information is increased. Many iterations of alignment and classification enable to generate class averages of several different views. These class averages are used for angular determination (4). When the angular orientation of the class averages is calculated, an initial 3D model is obtained by filtered back-projection. This model can be further improved in iterative refinement (5). Refinement includes 2D and 3D processing. The 3D model is projected and the projection images are used as reference to increase the alignment accuracy. Higher alignment accuracy increased the classification quality, and thus an improved 3D model can be obtained. Moreover, the calculation of the angular orientation is increased. Several iterations of refinement will stabilise a model of a maximum level of detail. 
a

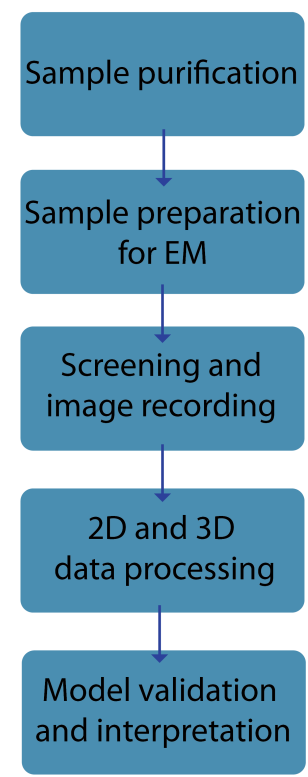

b
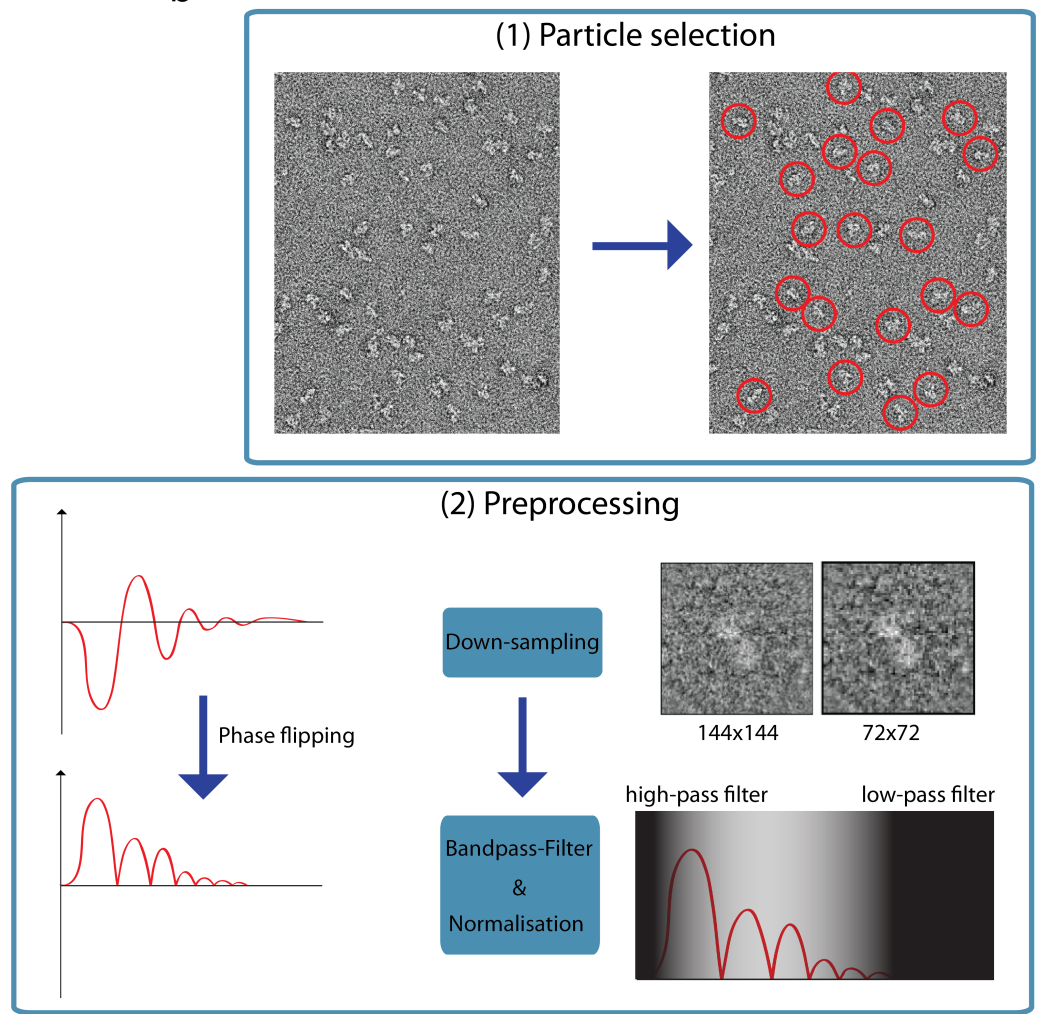

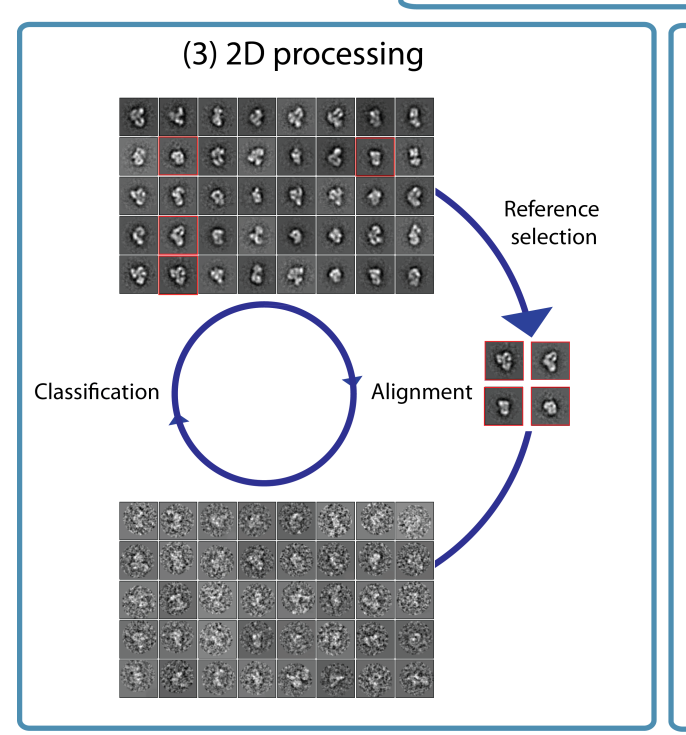

(4) Angular determination and 3D reconstruction
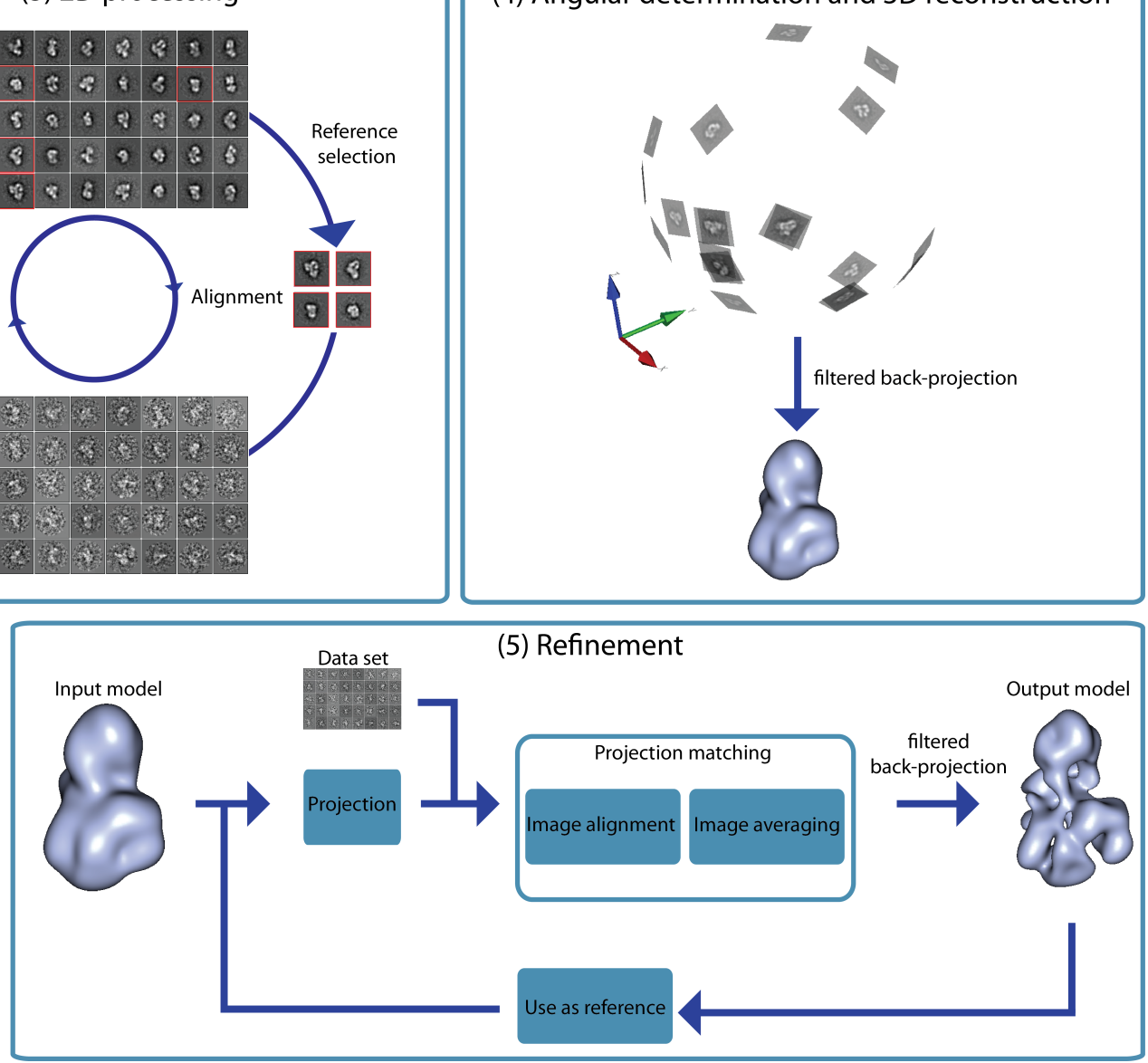
macromolecular complex due to inter- and intramolecular cross-links within the complex (further details in Section 2.1.4). To conduct microscopy, carbon-film-covered grids, small metal supporters used in EM, are prepared. Two different sample preparation techniques are possible: negative stain sample preparation (further details in Section 2.3.1) and cryo sample preparation (further details in Section 2.3.2). Negative stain sample preparation is reasonable for a first model of a complex of unknown structure. This is helpful for small objects $(<600 \mathrm{kDa})$, as negative stain provides a high contrast. Still, the achievable resolution is limited in negative stain. In most cases, higher resolution is aimed and can be achieved by cryo sample preparation. There, the sample is embedded in a thin layer of vitrified ice. The contrast, which is weaker than in negative stain, is generated from the sample itself, providing, in principle, atomic information. However, the low signal-to-noise ratio (SNR) and the convolution of the information by a contrast transfer function (CTF) complicate the gain of high-resolution information. Optimal conditions are defined by satisfying image contrast and thin and homogeneous embedding of the macromolecule in negative stain or vitrified ice. Depending on the sample preparation method and heterogeneity, datasets may vary between 20,000 and 2,000,0000 particle images. For negatively stained samples, relatively small datasets are sufficient. After electron micrographs are recorded, 2D and 3D image processing is performed. In Figure 1.3b the individual steps of image processing are summarised. Data processing can be divided into particle selection (1), preprocessing (2), 2D processing (3), angular determination, 3D processing (4) and refinement (5). A brief explanation of each step is given in the following section.

Individual macromolecular complexes are selected from the electron micrograph and stored as image stack. This can be performed manually, but improved semi-automated and automated selection tools are available (further details in 2.5.1.1). A preprocessing step is necessary to access all information stored within the images: Images are recorded at low defocus, the so-called Scherzer focus ${ }^{7}$, to increase the phase contrast, resulting in a image delocalisation and modulation with a contrast transfer function (CTF) (Lentzen, 2008). This can be partly corrected by CTF correction, where the phase flipping is reversed and the damping of high spatial frequencies (B factor) is reduced. Furthermore, the images are downsampled to decrease the data load and to increase the image contrast. Subsequently, the images are filtered and normalised. The image-filtering is necessary to remove unwanted information like image gradients or noise (further details about preprocessing in Section 2.5.1.2). The projection images are noisy and randomly oriented. By averaging similar views, one can increase the information content per image considerably, whereas the noise is reduced. To combine similar images, they need to be aligned. The combination of image

\footnotetext{
${ }^{7}$ Using a small defocus (Scherzer focus: $-1.2\left(C_{S} \lambda\right)^{1 / 2}$ ) it is possible to partly counteract the effects of the spherical aberration to enable an improved phase contrast. The fist zero crossing of the CTF is thereby shifted to higher spatial frequencies. In contrast the so-called Lichte focus is the point of minimised image delocalisation.
} 
alignment (shifting and rotating) and classification (generation of so-called class averages) creates different superimposed projection images of decent quality (further details about alignment in Section 2.5.1.4 and about classification in Section 2.5.1.6). Usually, this is done in an iterative 2D image processing loop by alternating alignment and classification. There, the output class averages are used as a reference to improve the alignment of the subsequent iteration (further details in Section 2.5.1.7). Once satisfying class averages are generated, they are used for angular determination and 3D reconstruction. In principle, the angular information is stored in each projection image and can be restored (see Fourier slice theorem in Section 2.5.2.1). By filtered back-projection, an initial 3D model can be obtained. This initial model needs to be refined to generate an improved 3D model. During refinement, the alignment accuracy can be increased iteratively. This higher accuracy effects all subsequent steps, so that the error in classification and angular determination is reduced and a more detailed 3D model will be obtained (further details about alignment in Section 2.5.1.8).

\subsubsection{Structural Validation}

Before a 3D model can be interpreted to answer a biological question, a validation is necessary. As wrong models can be derived easily, this is of special importance for de novo reconstructions. However, cryo EM is a young technique and so far no similar standards as

in X-ray diffraction are established. In 2012, a Validation task force (Henderson et al., 2012) was initiated to define common validation standards. One strategy is the so-called tilt validation, where the known angular relation between tilted and non-tilted images are used to validate a model (Henderson et al., 2011a). Nevertheless, tilt validation is difficult for small particles and can be of limited use for negatively stained particles. Another approach, the so-called "gold standard" demands that a dataset is split and that two independently refined 3D models of the same object are compared with each other (Scheres and Chen, 2012). High resolution structures can be validated by internal features, while the possibility of overfitting needs to be regarded. Additionally, a model can be validated by successful and precise fitting of available partial structural models into a high-resolution 3D EM map. Details of model validation and possible obstacles will be explained in the Chapter 2.

\subsection{Measuring Macromolecular Dynamics}

As outlined earlier (Section:1.1.4), molecular complexes are formed by the assembly of the corresponding subunits in a highly regulated manner. Accordingly, different subcomplexes 
with missing or additional bound factors can function as molecular switches or can even have alternating functions. That is how the cell organises network complexity with relatively few factors (Section: 1.1.5). Additionally, they are very dynamic in terms of flexibility and motion. Still, not every motional degree of freedom is functionally relevant. Consequently, it is important to carefully analyse dynamics in macromolecules. In the following chapter, the concepts of those dynamics, namely compositional and conformational heterogeneity, will be presented.

\subsubsection{Compositional and Conformational Heterogeneity}

Heterogeneity may represent interesting biological states of a complex but may also relate to sample degradation. Sample degradation is often the result of inadequate purification strategies and may be very limiting for high-resolution structure determination. However, different biological states may be of special interest to understand distinct functional states as shown recently in the case of CRM1 (Monecke et al., 2012). There are two types of heterogeneity, compositional and conformational heterogeneity. Compositional heterogeneity defines a state of a purified macromolecular complex with varying protein composition, while conformational heterogeneity specifies a complex which adopts different conformational states in solution (Figure 1.4). Both types of heterogeneity can be of functional interest or the result of inadequate sample treatment. Moreover, both types limit the attainable isotropic resolution of a complex and make the structural definition of a complex much more delicate, especially as long as no a priori knowledge of these complexes and their binding sites is given. Thus, very stable complexes with defined and stably bound subunits and a reduced dynamical landscape are preferred, as their datasets easier reach very high resolution. But there are many complexes of low stability and homogeneity. Many of those very transient, very flexible macromolecular complexes with several dynamically interacting subunits are of very high biological relevance, while only few studies are available. Analysing the Electron Microscopy Data Bank (EMDB), it can be shown that $86 \%$ of released high resolution maps show viruses, ribosomes or filaments. The 20S proteasome is very stable and inhomogeneous, while the 19S lid is more dynamic and less homogeneous. For instance, the 20S subunit of the proteasome had been solved long ago by X-ray crystallography, whereas the complete $26 \mathrm{~S}$ proteasome (with the 19S lid) remained unsolved until first cryo EM maps were obtained (Lasker et al., 2012a; Lander et al., 2012). Another example for obstacles related to heterogeneity can be found in case of the eukaryotic ribosomal expansion segments. Accordingly, the first high resolution structure of the eukaryotic ribosome (80S) (Ben-Shem et al., 2011; Jenner et al., 2012) was obtained later than that of the prokaryotic ribosome (70S) (Schuwirth 
et al., 2005). In summary, regarding the quantity of heterogeneous complexes, there is an increasing importance of methods dealing with these complexes.
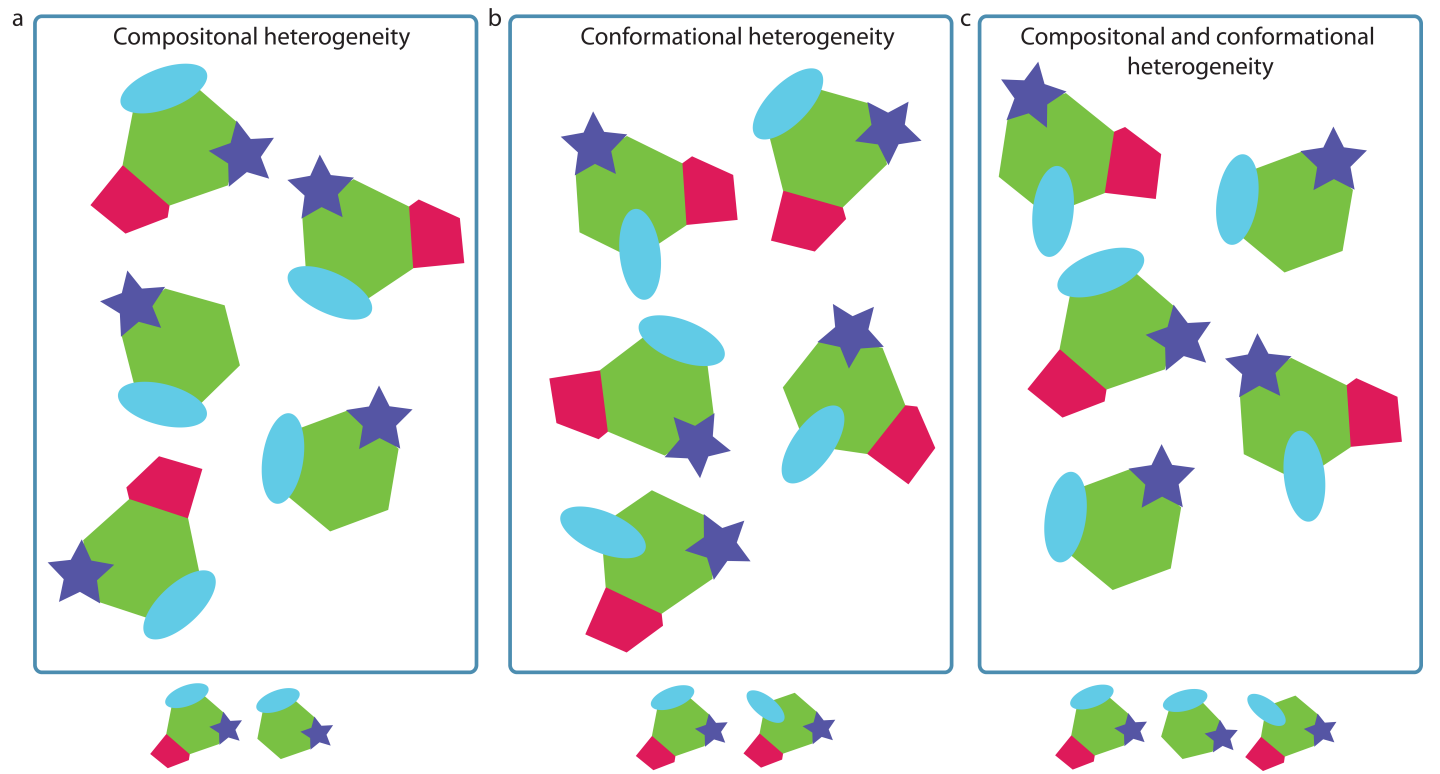

Figure 1.4: Compositional and Conformational Heterogeneity. Principally two forms of sample heterogeneities are distinguishable. a: Compositional heterogeneous macromolecular complexes represent a mixture of particles with and without at least one subunit (illustrated in red), while everything else is unaltered. b: Conformational heterogeneous macromolecular complexes can be divided into at least two different conformations. These conformational differences may be local (shown for the light blue subunit) or global. In the latter case, the overall appearance may change. c: Often, a mixture of compositional and conformational heterogeneity can be identified within a sample. In general, heterogeneity can be a consequence of sample degradation or may have a functional implication.

\subsubsection{Methods to Investigate Dynamics of Biomolecules}

As already mentioned before, structural methods like NMR, cryo EM and, to a certain extent, MS are able to analyse molecular dynamics:

1. NMR can define the conformational dynamics of peptides and proteins on a broad time-scale, starting from picoseconds, without a categorical upper time limit.

2. MS can answer questions regarding topology, composition and conformational dynamics in the millisecond time-scale.

3. Cryo EM can determine compositional and conformational dynamics of protein complexes $(>\sim 300 \mathrm{kDa})$ on a time scale of seconds to minutes.

In the following section, it will be focused on the measurement of dynamics by Single Particle EM. More details related to other methods can be found in Appendix section B.4. 


\subsubsection{Measuring Heterogeneity Using Single Particle EM}

As already stated before, single particle EM is suitable to resolve molecular dynamics. In transmission electron microscopy (TEM), each single particle image contains threedimensional information which is projected onto a two-dimensional plane. As the particles are bound in varying angular orientation, many different projections are obtained. Additionally, heterogeneous samples even vary within the same angular orientation. Thus, it is very tricky to disentangle heterogeneity from angular orientation without a reference 3D model. However, to some extent, the heterogeneity can already be examined in the raw image, the so-called micrograph, despite the low signal-to-noise ratio (SNR). In particular, this is helpful to define the quality of the sample by the amount of damaged particles and smaller fragments. This is already possible by observing the rough overall shape. Exemplary, disassembled $30 \mathrm{~S}$ and $50 \mathrm{~S}$ ribosomal subunits can be easily recognised in the micrograph. By visual inspection of raw images, only very obvious heterogeneity is noticeable, subtle heterogeneity can not be defined. In principle, in every processing step, meaning on micrograph level, on 2D class-sum level, on initial 3D level and on refined 3D level, a careful observation of possible heterogeneity is necessary. A reliable sign for local heterogeneity can be a local anisotropy with reduced resolution or lower local occupancy compared to the overall reached resolution. Image sorting is the main image processing tool to address heterogeneity within the dataset. Supervised and non-supervised sorting strategies allow to resolve compositional and conformational heterogeneity within a macromolecular complex (Section 2.5.3). In case a reference model is given, the sorting is simple. Reference models, either from earlier processing steps or by manual modifications based on prior knowledge, can be used to sort the dataset and generate an improved output model. Moreover, biochemical strategies are appropriate to reduce heterogeneity. Details related to the computational heterogeneity analysis in EM can be found in the Sections 2.5.3.

\subsection{Cohesin}

\subsubsection{Replication of Life}

Each cell is able to reproduce itself in a process called cell division, which is the basis for the amplification of life. An identical copy of the dividing cell is generated. The cell cycle is regulated by different types of cyclins, having periodic expression patterns (Malumbres and Barbacid, 2009). By activation of cyclin-dependent kinases (CDKs), cyclins control the onset of the corresponding next step in the cell cycle. CDKs, in turn, activate downstream 
effectors of the cell cycle, and define the framework for the functional cell division. The cell cycle can be separated into five different states according to Alberts et al. (2008):

1. $\mathrm{G}_{1}$ phase (Gap 1):

After a successful division, the cell enters $G_{1}$ phase. The cell increases in size and the cell metabolism is upregulated.

2. S-phase (Synthesis):

In S-phase, DNA replication is accomplished, starting at several loci in parallel, and the translated histones are repositioned into the nucleus.

3. $\mathrm{G}_{2}$ phase (Gap 2):

In $\mathrm{G}_{2}$ phase, the cell is prepared for mitosis. Division-specific proteins and RNAs are synthesised while the "endoplasmic reticulum" (ER) is melted down and cellular contacts are loosened.

4. M phase (Mitosis):

In M phase, the cell is ready for division. Almost all cellular processes that are not related to cellular division are paused.

5. $\mathrm{G}_{0}$ phase $(\operatorname{Gap} 0)$ :

In $G_{0}$ phase, the cell is in senescence, a quiescent state, where metabolism is reduced and cell cycle is paused. This is of special relevance for slowly and none dividing cells like nerve cells and heart muscle cells. Another example are stem cells, which can become inactive for decades.

$\mathrm{G}_{1}$ phase, $\mathrm{S}$ phase and $\mathrm{G}_{2}$ phase are combined in the so-called interphase, characterised by an active metabolism. As opposed to this cell, division is characterised by mitosis (karyokinesis) and cytokinesis, whereas mitosis can further be separated into five steps (Alberts et al., 2008):

1. Prophase:

In prophase, the chromosome is condensed and the mitotic spindle formation is initiated.

2. Promethaphase:

In promethaphase, phosphorylation of nuclear lamins results in the disintegration of the nucleus. Microtubules bound to the mitotic spindle enter the former nucleus and attach to chromosomal kinetochores in a bipolar fashion.

3. Metaphase:

Chromosomes are pulled towards opposite cellular ends, resulting in the alignment of the chromosomes on the equatorial plate. The "metaphase-to-anaphase checkpoint" ensures equal distribution of sister chromatids towards opposite ends. 
4. Anaphase:

In anaphase, sister chromatids are released from each other and are pulled towards opposite poles. The cell is elongated by microtubuli, so that the poles diverge from each other.

5. Telophase:

Finally, cytokinesis, the separation of the cell, is initiated while the nuclear membrane forms around the separated daughter chromosomes, constructing two new nuclei. Vesicles are redistributed between the forming cells, the ER and the Gogli apparatus are shaped and the cytokinesis is finished.

Within each nucleus of eukaryotic cells, the chromatin is condensed in a way that the DNA, corresponding to a total length of $1.8 \mathrm{~m}^{8}$, is packed onto histones to form a nucleosome, a structure of $11 \mathrm{~nm}$ in diameter. The nucleosome, an octameric protein complex, is the basic repetitive packaging unit for chromatin in the cell. These nucleosomes are compacted, to form a $30 \mathrm{~nm}$ thick interphase chromatin fiber. However, in metaphase, these fibers fold into a hierarchy of multiple loops and coils, a so-called supercoil. Early on, it became obvious that histones alone are not sufficient to establish the condensed structure and organisation of the chromosomes, especially in metaphase.

\subsubsection{Identification of Cohesin and Condensin}

In 1977, it was shown that, after the removal of histone proteins, metaphase chromosomes still contain 30 different proteins, holding the chromosome together in a loop-like shape. These proteins were grouped into Sc1 and Sc2 (Scaffold), with $170 \mathrm{kDa}$ and $135 \mathrm{kDa}$, respectively (Adolph et al., 1977; Paulson and Laemmli, 1977). Sc1 was later identified as Topoisomerase II (Gasser et al., 1986), whereas the nature of Sc2 proteins stayed mysterious until the 1990s (Saitoh, 1994). In 1985, the name $\mathrm{SMC}^{9}$ was introduced for a gene required for the stability of minichromosomes in S. cerevisiae (Larionov et al., 1985). Later, it was related to MukB from E. coli, harbouring an ABC-like ATPase domain and coiled-coil domains (Niki et al., 1991, 1992). As bacteria, lacking nucleosomal organisation, still have their chromosomal structure very regularly arranged, soon those proteins were linked to the previously identified Sc2 proteins. Accordingly, Xenopus extracts, harbouring orthologs of these Smc proteins (XCAP-C/E), were shown to be able to condense chromatin in vitro (Hirano and Mitchison, 1994). Moreover, all these proteins (Smc2, cut3, Sc2, XCAP-C/E) were linked within the SMC family. Smc1, Smc3, Scc1 and Scc2 ${ }^{10}$ were identified to be responsible for chromatid cohesion before separation in anaphase and, therefore, named

\footnotetext{
${ }^{8}$ Actually, this is only true if all individual chromosomes of a diploid cell are linked together.

${ }^{9}$ Structural maintenance of chromosomes

${ }^{10}$ Sister chromatid cohesion, alternatively Rad21 for Scc1
} 
cohesins (Michaelis et al., 1997). Still, the functional organisation of those proteins was unclear. Later, Smc1, Smc3, Scc1 and SA11 ${ }^{11}$ were found in animal cells to form a $14 \mathrm{~S}$ complex (Losada et al., 1998; Toth et al., 1999). Henceforth, this cohesin complex will be referred to as the cohesin tetramer.

Today, Smc proteins are known to be the key players in chromosomal condensation and segregation. Smc proteins contribute to two different but closely related families of protein complexes, cohesin and condensin complexes, though the first can only be found in eukaryotes (Peters et al., 2008; Hagstrom and Meyer, 2003). Condensins and condensin-like complexes are found in all three domains of life and are suggested to be more ancient than cohesins (Cobbe and Heck, 2004). Condensins are essential for the substantial reorganisation and compaction of chromosomes during mitosis (Hirano et al., 1997). Condensin II condenses chromatin during interphase, as it is located within the nucleus. Condensin I, located in the cytosol, can only access chromatin when the nuclear envelope breaks down, condensing together with condensin II chromatin, to form the particular shape of mitotic chromosomes (Hirota et al., 2004). Cohesin was identified to hold sister chromatids together, until the onset of anaphase, ensuring the equal distribution of sister chromatids to the opposing cell poles (Michaelis et al., 1997). Mutations of cohesin were related to precocious sister chromatid segregation and aneuploidy (Michaelis et al., 1997). Furthermore, cohesin plays an important role in meiosis. It ensures separation of homologue chromosomes in meiosis I by releasing cohesin from the chromosome arms (Buonomo et al., 2000). Even more, it guarantees separation of sister chromatids in meiosis II by releasing the remaining cohesin at the centromeres. Substitution of Scc1 by its paralog Rec8 is essential for this role in meiosis (Klein et al., 1999; Watanabe and Nurse, 1999). Moreover, cohesin was shown to play an important role in DNA damage control and in transcriptional control. In yeast, cohesin accumulates on areas containing double strand breaks (DSB) (Unal et al., 2007; Strom et al., 2007), and in human cells, the depletion of sororin, a direct regulator of cohesin, caused severe defects in the repair of DSBs (Schmitz et al., 2007).

Cohesin defects are related to a few diseases like Cornelia de Lange syndrome and Trisomy 21 (Liu and Krantz, 2008; Deardorff et al., 2012). The age-related missegregation can be induced by defects in sister chromatid cohesin. However, other chromosomal missegregations are believed to occur at the same frequency but lack a phenotype as most of them are lethal.

Generally, in eukaryotes, heterodimers of Smc are formed, allowing the specification of each Smc, while in prokaryotes only Smc homodimer are formed (Cobbe and Heck, 2004). Additionally, both condensin and cohesin contain kleisin subunits of different type

\footnotetext{
${ }^{11}$ In vertebrates, there are three homologues, SA1, SA2 and SA3, the latter being specific to meiosis.
} 
(Nasmyth and Haering, 2005). In the present work onlx the mitotic human Scc1 is of interest. In yeast, Scc3 was shown to be kleisin-associated and to be essential for sister chromatid cohesin (Haering et al., 2002; Kulemzina et al., 2012). There are several different orthologs of kleisin-associated proteins in vertebrates like SA1 and SA2 (Losada et al., 2000; Sumara et al., 2000), both relevant to mitotic cells and STAG3, which is specific to meiosis and interacts with the also meiosis-specific kleisin Rec8 (Klein et al., 1999; Watanabe and Nurse, 1999). Recently, it was shown that cohesin-SA2 is the main complex co-recruited to DNA damage sites, but not cohesin-SA1 (Kong et al., 2014). Furthermore, there are several interaction partners of the cohesin tetramer, like Pds5, Wapl ${ }^{12}$ and sororin. In Table 1.2, relevant components and interaction partners of human cohesin are listed:

Table 1.2: Composition and Interaction Partners of Human Cohesin Complex Isoforms that are expressed specifically in meiosis are highlighted $\left(^{*}\right)$.

\begin{tabular}{|c|c|c|c|}
\hline protein family and description & generic name & molecular weight (kDA) & gene name \\
\hline \multirow[t]{3}{*}{ SMC } & Smc1 & 143 & SMC1A \\
\hline & $\mathrm{Smc} 1 \beta^{*}$ & 144 & SMC1B \\
\hline & Smc3 & 140 & SMC3 \\
\hline \multirow[t]{3}{*}{ Kleisin } & Scc1 & 72 & $\operatorname{Rad} 21$ \\
\hline & Rec $8^{*}$ & 63 & Rec8 \\
\hline & Rad21L* & 63 & $\operatorname{Rad} 21 L$ \\
\hline \multirow[t]{3}{*}{ Kleisin associated } & SA1 & 145 & STAG1 \\
\hline & SA2 & 138 & STAG2 \\
\hline & $\mathrm{SA}^{*}$ & 139 & STAG2 \\
\hline \multirow[t]{2}{*}{ Regulatory factor } & Pds5A & 151 & PDS5A \\
\hline & Pds5B & 165 & PDS5B \\
\hline Activator of DNA dissociation & Wapl & 140 & WAPAL \\
\hline Wapl inhibition & Sororin & 28 & CDCA5 \\
\hline \multirow[t]{2}{*}{ Cohesin loading } & Scc2 & 316 & NIPBL \\
\hline & Scc4 & 70 & MAU-2 \\
\hline Kleisin cleavage & Separase & 233 & ESPL1 \\
\hline Separase inhibition & Securin & 22 & PTTG1 \\
\hline \multirow[t]{2}{*}{ Smc3 acetyltransferase } & Esco1 & 95 & ESCO1 \\
\hline & Esco2 & 68 & ESCO2 \\
\hline Smc3 deacetylase & Hdac8 & 42 & HDAC8 \\
\hline
\end{tabular}

\subsubsection{Biochemistry of the Cohesin Tetramer}

Today, it is known that euploid chromosome segregation depends on sister chromatid cohesion, which is mediated by cohesin tetramer (Peters et al., 2008). Smc forms a globular and a hinge domain, which are interrupted by a coiled-coil segment. The Smc hinge strongly attaches to an adjacent Smc hinge, allowing the formation of a dimer (Huis in 't Veld, 2013). The globular domain is shaped by the back-folded $\mathrm{N}$ - and C-terminal domain and arranges into a ABC-ATPase with a relatively low ATPase activity (Lowe et al., 2001). The ABC-ATPase-specific Walker A (P-loop) and Walker B motifs are separated by 900

\footnotetext{
${ }^{12}$ alternatively, Rad61 or Wpl1 in different saccharomyces species
} 
aa due to two interjacent coiled-coil motifs and the hinge-related sequence (Lowe et al., 2001). The ABC-ATPases bind ATP, along with a stabilised $\mathrm{Mg}^{2+}$ ion and two water molecules. This is facilitated by the Walker A motif, the Walker B motif, the signature motif and the Q-loop (Hopfner et al., 2000; Lowe et al., 2001; Haering et al., 2004). In the case of cohesin, the interaction of two nucleotide binding domains (NBDs) forms a composite nucleotide binding pocket, where two nucleotides can be sandwiched, each by the signature motif of the one Smc and the Walker A and B domains of the other Smc. The binding of ATP was shown to induce this sandwich formation, and to bring the Smc globular domains together, while hydrolysis is believed to reverse this dimerisation (Haering et al., 2004). It is yet vague whether there is a cooperative effect of the two nucleotides. Obviously, by corporate action of a hinge domain and a globular domain, two Smc proteins are able to establish a ring-like dimeric structure, which was validated by electron microscopy (Melby et al., 1998; Anderson et al., 2002; Haering et al., 2002). Even more, the Smc1 and the Smc3, together with Scc1, form a tripartite ring which spans $45 \mathrm{~nm}$ and is independent from the ATP binding state (Weitzer et al., 2003). It was recently shown that Scc1 bridges the two Smc proteins by binding asymmetrically with the N-terminus to the coiled-coil region of Smc313 (Gligoris et al., 2014; Huis in 't Veld et al., 2014), whereas the C-terminus interacts with the globular domain of Smc ${ }^{14}$ (Haering et al., 2004). Interestingly, the NHD is composed of the N-terminal 102 aa, whereas the winged-helix domain (WHD) is shaped by the C-terminal 80 aa, while 449 aa seem to remain largely unstructured and bridge a huge distance of up to $30 \mathrm{~nm}$. This huge ring-like structure early on led to the hypothesis that cohesin may topologically entrap either one or two DNA strands to fulfil its biochemical role, the so-called ring model (Weitzer et al., 2003). Non-entrapment-depending attachment seems unlikely, although it was debated (Huang et al., 2005; Nasmyth, 2011). Depending on the model, either one (Weitzer et al., 2003; Ivanov and Nasmyth, 2005, 2007; Haering et al., 2008) or two (Huang et al., 2005; Nasmyth, 2011) cohesin complexes are needed to attach sister chromatids. The latter model depends on the interaction of two cohesin rings (Nasmyth and Haering, 2005).

There are several reasons to favour the cohesin ring model. In S. cerevisiae, it was shown that cohesion depends on the state of cleavable minichromosomes or cleavable cohesin tetramer (Ivanov and Nasmyth, 2005, 2007). In the cleaved state of either minichromosomes or cohesin, cohesion is prevented, arguing that only DNA binding cannot be the principle of cohesion. Additionally, covalently linked interfaces of cohesin can rescue cohesion loss in denaturing condition (Haering et al., 2008). Recently, it was shown that a Scc1 exit gate mutant, unable to close the $\mathrm{NHD}^{15}$-Smc3 interface, failed to stabilise chromatin interaction and to support sister chromatid cohesion (Huis in 't Veld et al., 2014). Still, there are

\footnotetext{
${ }^{13}$ This N-terminal domain is called the N-terminal helical domain (NHD).

${ }^{14}$ This C-terminal domain is the so-called winged-helix domain (WHD).

${ }^{15} \mathrm{~N}$-terminal helical domain
} 
different models proposed, like the snapping by cohesin dimers or the filament-like bracelet formation (Nasmyth, 2005; Huang et al., 2005; Nasmyth, 2011).

a

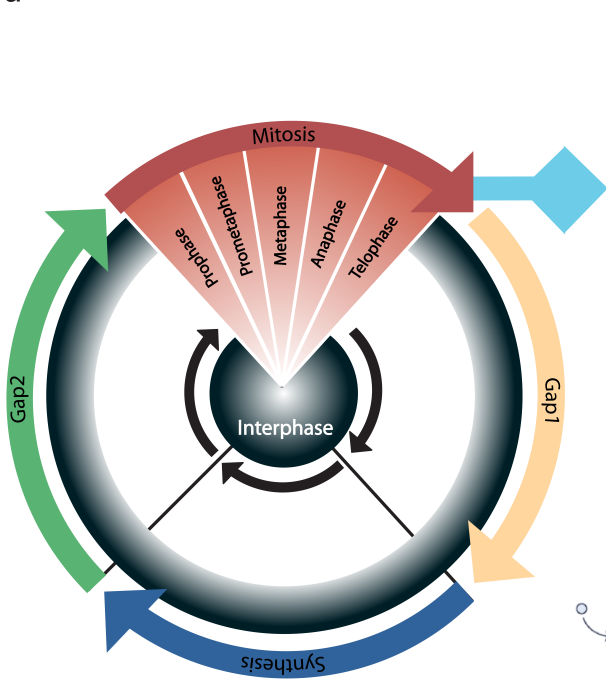

b

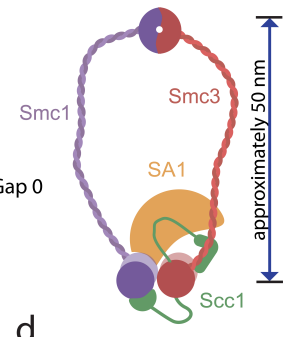
d
C

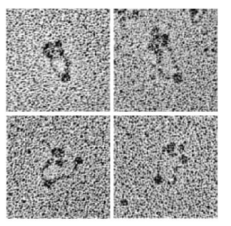

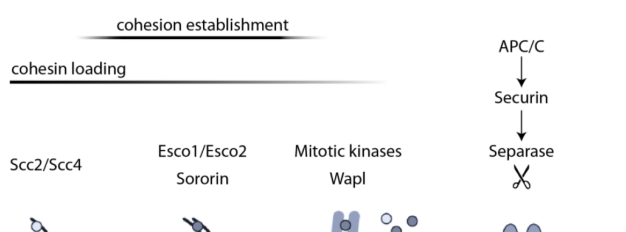

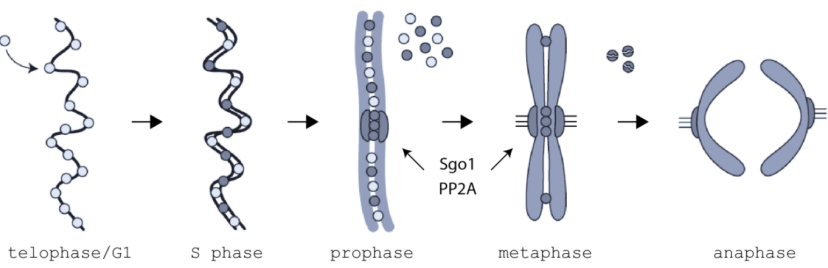

Figure 1.5: Biochemistry of Cohesin. a: Scheme of the cell cycle according to the summary in Section 1.5.1. Gap1 in yellow; Synthesis in blue; Gap2 in green; Mitosis in red and Gap0, the senescent phase, in light blue. Gap1, synthesis and gap2 are summed up as interphase. Mitosis is subdivided into prophase, prometaphase, metaphase, anaphase and telophase. b: The schematic representation of cohesin shows the long coiled-coil arms of Smc1 and Smc3. The hinge domain at the top is approximately $50 \mathrm{~nm}$ apart from the globular head domains which are bound to SA1 and Scc1. The scheme was generated by Pim Huis in't Veld and was modified with permission c: An exemplary representation of rotary shadowed endogenous human cohesin shows the typical ring shape appearance (Anderson et al., 2002). d: Cohesin loading, cohesion establishment and cohesin removal during cell-cycle. After replication, cohesin becomes stably bound. Light grey circles represent the pool of dynamically bound cohesin, while dark grey circles represent the stably bound cohesin, which is mainly located at the centromer. Dynamically bound cohesin is removed during prophase pathway, while stably bound cohesin can only be removed upon APC/C-dependant cohesin cleavage by separase. The figure was adapted with permission by Pim Huis in't Veld (Peters et al., 2008).

\subsubsection{Interaction Partners and Regulation of Cohesin}

Functional cohesion requires a regulated loading and unloading of cohesin onto chromatin. Several regulative binding partners of cohesin, like Pds5, Wapl and sororin orchestrate this process (reviewed in Peters et al., 2008; Peters and Nishiyama, 2012). Loading of cohesin onto DNA in vertebrates happens in telophase, before DNA replication. The loading is facilitated by a separate loading complex consisting of Scc2 and Scc4. In Xenopus egg extracts, the recruitment of Scc2/Scc4 to a pre-replicative complex (pre-RC) is elementary for loading of cohesin onto chromatin (Gillespie and Hirano, 2004; Takahashi et al., 2004). This is initiated via the protein kinase Cdc7 and one of its activating subunits Dbf4 or 
Drf1 (Takahashi et al., 2008). Whether this pre-RC is essential for cohesion in mammalian cells is not known, but the complex assembling time is consistent with the time span of cohesin loading (Méndez and Stillman, 2000). While the N-terminal half of Scc2 and full length Scc4 are sufficient to bind to pre-RCs, the C-terminal HEAT repeat of Scc2 seems to interact with cohesin (Takahashi et al., 2008). Significantly, there are several indications suggesting a role of the hinge domain in cohesin loading, including a transient opening of the hinge. Loading of cohesin onto DNA is prevented by permanently linking the hinge-domain of Smc1/3 together, whereas a fusion of Smc1-Scc1-Smc3 has no effect on loading (Gruber et al., 2015). In this context it is interesting that the ATPase function plays a role in cohesin loading, as DNA association depends on ATPase activity (Hu et al., 2011). Cohesin, which is incapable in ATPase hydrolysis, can still be co-localised with Scc2/4 at the centromeres, maybe resembling a transition state of cohesin loading before stable entrapment is established. Supposing that Scc2/4 interacts with the cohesin hinge domain, it might be that ATP hydrolysis modulates affinity between Smc1 and Smc3 hinge domain.

Topological entrapment of DNA argues for a relatively long residence time of cohesin on chromatin, compared to other DNA-interacting proteins. Indeed, the average residence time of 10-20 minutes in G1 phase, as identified by FRAP, is high compared to the interaction time of transcription factors in the range of seconds (Gerlich et al., 2006). Considering the decade-long stabilisation of chromatid in stem cells and prophase-I-arrested oocytes, this time frame is still low and would not explain the strong stabilisation in the two mentioned cell types. In G2 phase, one third of cohesin was found to be stabilised, with very long average residence times of at least several hours. This stabilisation happens during S phase and can explain the extreme enduring stabilisation of sister chromatid in specific cells like oocytes (Tachibana-Konwalski et al., 2010). This stabilisation depends on previously identified N-terminal Smc3 acetylation (Nishiyama et al., 2010). The acetyltransferase Eco1 in yeast ${ }^{16}$ was shown to acetylate K112 and K113 of Smc3 at the top of the ATPase head, referred to as KKD strand (Rowland et al., 2009). This KKD strand seems to have a role in cohesin release from DNA, depending on Wapl, Pds5 and Scc3 regulatory subunits. Acetylation of K112 and K113 by Eco1 is able to inhibit this activity and stabilise cohesin associated with chromatin. Even more, Smc3 R61 links the KKD strand to the Smc3's ATP binding pocket, raising the question if the acetylation state may regulate ATPase activity and vice versa (Gligoris et al., 2014). Indeed, a link was shown between acetylation and ATPase activity by a rescue mutant (acetylatable lysines) of an otherwise lethal effect of ATP-hydrolysis mutant (Heidinger-Pauli et al., 2010). Also, acetylation is not possible for mutants that cannot hydrolyse ATP or have an altered Smc1-Smc3 hinge interface (Kurze et al., 2011; Hu et al., 2011). This again raises the question of how DNA loading,

\footnotetext{
${ }^{16}$ Eco1 is equivalent to vertebrate Esco1 and Esco2.
} 
ATPase activity and acetylation are linked. Recently, cohesin's ATPase activity was shown to couple cohesin loading onto DNA with Smc3 acetylation (Ladurner et al., 2014). In human cells, the binding of sororin may stabilise cohesion on centromeres after Smc3 acetylation occurs.

\subsubsection{Cohesin Unloading from DNA}

Just as cohesin loading must be regulated, there needs to be a mechanism to control cohesin unloading. Originally only one mechanism of cohesin removal was identified. This is based on cohesin cleavage by separase (Uhlmann et al., 1999, 2000). In contrast, in metazoans, a vast amount of cohesin is spared from cleavage, as it is removed by a non-degradative process, the prophase pathway (Waizenegger et al., 2000). Only a small fraction of cohesin bound to the centromeres ${ }^{17}$ is shielded from prophase pathway and cleaved by separase at metaphase-to-anaphase transition.

The prophase pathway is associated with the release activity regulated by Pds5 and Wapl, while the latter can be understood as an antagonist of sororin. Recently, it was shown that the interface between Smc3 and Scc1 is the exit gate to release chromatin in prophase pathway and that the release is controlled by Wapl (Huis in 't Veld et al., 2014). For a long time it was debated what kind of opening mechanism terminates the topological entrapment of sister chromatids. The process is initiated by the phosphorylation of SA2 and Scc1 by Plk1 (Sumara et al., 2002; Hauf et al., 2005). Also, sororin can be phosphorylated by Cdk1 and Plk1. It is speculated that, by phosphorylation of sororin, the protective effect, ensuring long-term cohesion of acetylated cohesin, is inactivated and Wapl can promote dissociation (Dreier et al., 2011; Zhang et al., 2011). Accordingly, a phosphatase (PP2A) is recruited to the centromeres to dephosphorylate cohesin locally and prevent Wapl-induced cohesin opening. PP2A itself is recruited to Shugosin (Sgo1), which associates with centromeres in an Aurora B kinase-dependent manner (Riedel et al., 2006) (reviewed in Peters et al., 2008). Thus, the Sgo1-PP2A complex can protect centromeric cohesin until separase is activated at the onset of anaphase. Lately, it was proposed that the combined action of sororin and Sgo-PP2A prevents Wapl binding at centromeres (Liu et al., 2013). The N-terminal part of Wapl competes on one site of Pds5 with sororin by FGF stretches. Sgo1-PP2A competes with Wapl for the same binding site on SA2 (Shintomi and Hirano, 2009; Nishiyama et al., 2010). Still, the detailed sequence of prophase pathway and centromeric protection from prophase pathway is not fully understood. It is open how acetylation and phosphorylation, as well as sororin and Wapl action, intercommunicate. Interestingly, a knock-out of Eco1 is only viable if additionally

\footnotetext{
${ }^{17}$ This is actually the reason for the mitotic chromosomal X-shape.
} 
Wapl is depleted (Chan et al., 2012). Thus, the weakening of Smc3-Scc1 interaction by Wapl is inhibited by Eco1-dependent acetylation of Smc3.

The separase-dependent cohesin opening mechanism is better understood. It is known that there are two separase cleavage sites $\left(\operatorname{Arg}^{172} ; \mathrm{Arg}^{450}\right)$ in human Scc1, opening cohesin upon cleavage (Hauf et al., 2001). This process is regulated by the action of the anaphase promoting complex (APC/C), an E3 ubiquitin ligase, which labels cyclin B and securin for degradation by the proteasome (reviewed in Peters, 2002). Thus, separase, otherwise bound to securin and inactivated by phosphorylation, is activated and can remove chromatinbound cohesin through proteolysis of Scc1. The activation of APC/C is tightly controlled in mitosis and is only allowed upon bi-orientation of chromosomes on the mitotic spindle in the metaphase-to-anaphase transition. While in budding yeast, most cohesin is removed due to separase, in mammalian cells, this affects only the centormeric cohesin. Most of the cohesin was removed earlier due to the prophase pathay. (reviewed in Peters et al., 2008; Peters and Nishiyama, 2012). Accordingly, loading of cohesin happens earlier (telophase) compared to the equivalent process in budding yeast (G1 phase), where the free Scc1 pool needs to be refreshed.

\subsubsection{Structural Insights into Cohesin}

The cohesin tetramer (0.5 MDa) consists of four subunits, namely Smc1, Smc3, Scc1 and SA1. Smc1 and Smc3 are connected at one end by a hinge domain and are bridged by Scc1, a kleisin family member, at the globular end, the Smc1/Smc3 nucleotide binding domain (NBD). The hinge and NBD are connected via coiled-coils. In Smc, the NBD is shaped by the N- and C-terminal ends, whereas the coiled-coil has a back-folded and thus antiparallel nature (Haering et al., 2002). The hinge domain possesses a strong affinity $\left(\mathrm{K}_{\mathrm{d}}=20 \mathrm{nM}\right)$ (Kurze et al., 2011), whereas the interaction of the NBD of Smc1/3 is relatively weak and depends on the ATP/ADP binding status (Hu et al., 2011). The coiled-coils contribute approximately $50 \%$ of the total mass of one Smc, reflecting a length of $50 \mathrm{~nm}$. Scc1, a $72 \mathrm{kDa}$ protein, which is largely unstructured, asymmetrically binds to Smc1 and Smc3. The N-terminal helical domain (NHD) interacts with the coiled-coil of Smc3, close to the onset of the Smc3 NBD (Gligoris et al., 2014), while the C-terminal winged-helix domain (WHD) directly interacts with the globular head of Smc1 (Haering et al., 2004). The middle part of Scc1 has been shown to interact with SA2 (an isoform of SA1) and needs to bridge a long distance to form the tripartide ring structure together with the C-terminal and N-terminal Smc-bound part of Scc1 (Scc1 C-terminus: Smc1; Scc1 N-terminus: Smc3) (Hara et al., 2014). SA1 consists mainly of HEAT repeats and predicted unstructured regions of 100 aa $\mathrm{N}$ - and 200 aa C-terminal (Losada et al., 2000; Sumara et al., 2000). It has been shown to associate with Scc1. HEAT repeats, named after 


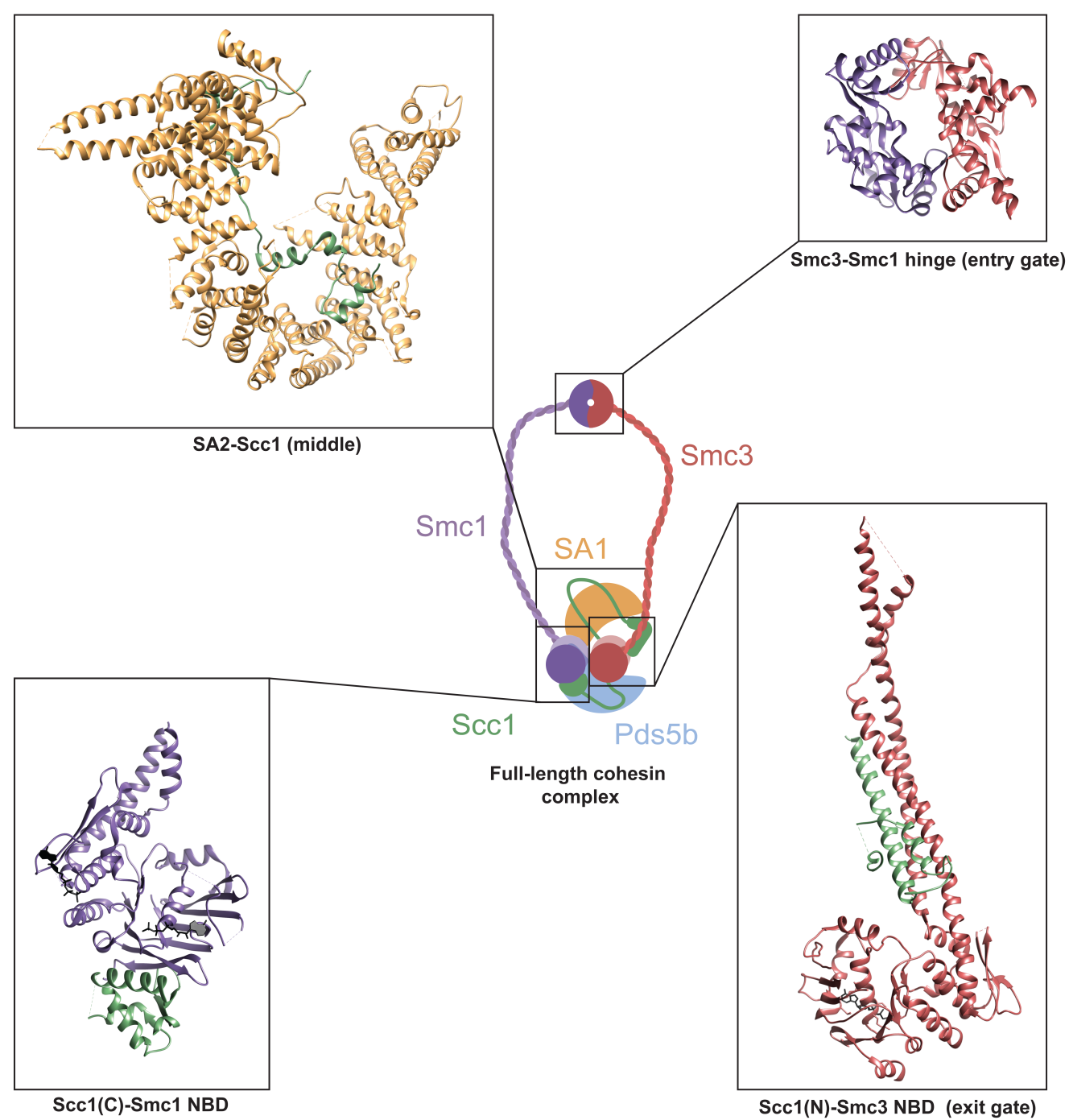

Figure 1.6: Relevant Structural Models of Cohesin Subunits. In the middle, the scheme of cohesin is illustrated. Areas where structural data is available are boxed and the respective structural models are highlighted. The subunits are depicted in different colours : Smc1 in purple; Smc3 in red; SA1 in yellow; Scc1 in green and Pds5 in light blue. At the top left, SA2 bound to a fragment of Scc1 (PDB entry: 4PJU [human]) is shown, and at the top right, the structure of the hinge domain (PDB entry: 2WD5 [mouse]) is represented. SA2 possesses a very high sequence identity to SA1. The $\alpha$-helical HEAT repeat motive is visible. At the bottom, the two NBD (Smc1/3) models (PDB entry: Smc1: 1W1W [yeast]; Smc3: 4UX3 [yeast]) are depicted with the asymmetric Scc1 binding sites. At the N-terminus (N-terminal helical domain (NHD)), Scc1 interacts with the coiled-coil of Smc3 in proximity to the NBD, and at the C-terminus, Scc1 (winged-helix domain (WHD)) interacts with the Smc1 NBD (Haering et al., 2004; Kurze et al., 2011; Hara et al., 2014; Gligoris et al., 2014, 1W1W, 2WD5, 4PJU, 4UX3, respectively). 
the initial letter of the proteins were they where identified ${ }^{18}$, form a big family of protein motifs important for hydrophobic interactions. They are 50 aa long and are formed by two antiparallel $\alpha$-helices (A,B) per repeat. The A helix often contains a strong curvature. Through several repeats, a hydrophobic core is formed, a so-called solenoid $\alpha$ - $\alpha$ superhelix. This superhelix is extreme flexible, allowing the adaptation for many different binding partners. This explains the evolutionary success of this motif and why it is found in importins $\alpha$ and $\beta$, where this flexibility is mandatory (Andrade et al., 2001a,b). Recently, SA2 was shown to contain 17 HEAT repeats, forming a curved dragon-shaped structure (Hara et al., 2014). Interestingly, not only SA1/2 but also further cohesin interacting proteins contain HEAT repeats, namely Pds5 and Wapl (Panizza et al., 2000; Ouyang et al., 2013). Pds5, a regulatory subunit of the cohesin tetramer, has been shown to bind to Scc1. Several interactions between Wapl N-terminus and Pds5 are identified (see Huis in 't Veld, 2013, 5.4.4). Even more, sororin is believed to interact with Pds5 by its FGF stretches (Nishiyama et al., 2010). Two homologues of Pds5, namely Pds5A and Pds5B, are identified in vertebrates and are suggested to be functionally redundant. $72 \%$ of the Pds5B sequence is identical, especially the N-terminal 1140 aa, whereas the C-terminus is very divergent. In the N-terminal part, Pds5 contains two HEAT repeats clusters (Losada et al., 2005; Panizza et al., 2000). Wapl interacts strongly with Pds5 and competes with Sgo1-PP2A for a SA2 binding site (Shintomi and Hirano, 2009; Rowland et al., 2009). It contains repetitive motifs like HEAT and ARM motifs, while the latter consists of three $\alpha$-helices (H1, H2 and H3). The first two $\alpha$ helices (H1, H2) correspond to the HEAT A-helix. ARM repeats stack into a solenoid through hydrophobic interactions, comparable to those in HEAT motifs. Compared to Pds5, Wapl is only poorly conserved (Ouyang et al., 2013).

The first published structure related to cohesin in yeast was received in 2004, containing the C-terminal WHD of Scc1 bound to a pair of $\beta$-sheets in Smc1's ATPase head (53; PDB:1W1W) (Haering et al., 2004). Still, as the sequence of Scc is not symmetric, at that time it remained unclear if the interaction of N-terminal Scc1 with Smc3 is similar. In 2011, the structure of the Mus musculus hinge, the proposed DNA entry gate, was solved, illustrating a pseudo-symmetric heterodimeric torus containing a small, positively charged channel (54; PDB: 2WD5) (Kurze et al., 2011). In 2014, two important parts of cohesin were solved. First of all, the structure of human SA2 bound to a fragment of Scc1 was solved, illustrating the overall topology of SA2 and the mechanism by which Wapl and Sgo1-PP2A antagonise each other (55; PDB: 4PJU) (Hara et al., 2014). Very recently, the structure of Smc3 with a N-terminal fragment of Scc1 was solved, explaining the mechanism of the DNA exit gate during prophase pathway (56; PDB: 4UX3) (Gligoris et al., 2014; Huis in 't Veld et al., 2014).

\footnotetext{
${ }^{18} \mathbf{H u n t i n g t i n}$, Elongation factor 3, PR65/A, and TOR
} 
So far, no overall structure, neither of the cohesin tetramer alone nor of the cohesin tetramer with regulative interaction partners, has been solved. Still, some crystal structures of certain areas of the complex are available, which contributed to the structural understanding of cohesin so far. Based on these structures, functional models have been proposed, which await to be validated by further structural data. Bringing those so far uncoupled available data together in an overall map would increase the knowledge and understanding of cohesin function immensely. For example, it is not yet possible to localise where Pds5 and Wapl bind to cohesin. 


\subsection{Prokaryotic Ribosome (70S)}

\subsubsection{Research History of Prokaryotic Ribosome (70S)}

The history of ribosome research is extensive and complex, so that only a brief summary of relevant steps important for structural and dynamical understanding can be given. Ribosome research started with the identification of RNA-rich particles, so-called "Palade particles" or "microsomes", in proximity to the endoplasmic reticulum (Palade, 1955). Later, these "Palade particles" have been related to protein synthesis (Crick et al., 1961), and were renamed "ribosomes"19. Structural investigation of the ribosome remained restricted to only a few techniques, whereas electron microscopy played a main role. Still, electron microscopy enabled only low resolution structures. In 1976, a first structure was proposed, derived from the analysis of negative stained 2D projection images (Lake, 1976). In 1995, improvements in EM, particularly in cryo sample preparation and image processing, resulted in an EM map of $23 \AA$. 2D electron crystallography was used to define the protein exit tunnel at low resolution (Yonath et al., 1987). However, it remained impossible to generate crystals of the ribosome for many decades. Crystallography had been successful only for single protein subunits of the ribosome, like L6 (Golden et al., 1994). Chemical probing was used to elucidate secondary structure motifs of RNAs like in the case of ribosomal 23S RNA (Leffers et al., 1988) or the tRNA interactions with 23S RNA (Moazed and Noller, 1989a).

Finally, in 2000, the first high-resolution crystal structure of the 50S subunit was published (Ban et al., 2000). This breakthrough was only possible by the development of uncommon crystallisation concepts of highly active ribosome, which were purified from extremophile organisms. The first crystals of the 50S subunit were derived in the early 1980s, and it took many years to tackle the many obstacles (see Rodnina, Marina V., Wintermeyer, Wolfgang, Green, 2011, Chapter 1.1). Especially, trapping the ribosome in specific conformations by binding factors, substrates or inhibitors yielded fruitful results (Yusupov et al., 2001; Korostelev et al., 2006). As radiation damage easily caused crystal decay, the imaging at cryogenic temperature was developed (Hope et al., 1989). An immanent problem arose from the loss of phase information during spot recording. Two alternative strategies helped to solve this problem. By combining EM maps ${ }^{20}$ for molecular replacement (Ban et al., 1998) with heavy atom clusters for multiple isomorphous replacement and anomalous scattering approaches, the first reliable high resolution map of the 50S subunit was determined (Ban et al., 2000). Shortly after, high resolution maps of the 30S subunit (Schluenzen et al., 2000; Wimberly et al., 2000) and the complete 70S ribosome were defined (Schuwirth et al.,

\footnotetext{
${ }^{19}$ In 1974, the Medicine Nobel Prize was awarded for the discovery of ribosomes.

${ }^{20}$ For EM, the phase problem, which is limiting in X-ray diffraction, does not exist.
} 
2005). Still, many processes regarding the dynamics of the ribosome, are analysed by a combination of high-resolution cryo EM, X-ray diffraction and biochemical approaches, like smFRET and pre-steady-state kinetics.

For four decades, it was assumed that decoding and peptide bond formation were performed by ribosomal proteins. Already in the late 1990s, it was shown that peptide bond formation and conjugation of amino acids can be accomplished in vitro by selected oligonucleotides (Zhang and Cech, 1997; Illangasekare et al., 1995). But it was only after the ribosomal structure was solved that the ribosome was understood as such a ribozyme. Only the N-terminal tail of L27 is in proximity to the Peptidyl transferase center (PTC) and may stabilise the P-site tRNA (Steitz, 2008; Voorhees et al., 2009). Today, the ribosomal core is understood to be related to a very ancient system, namely the so-called proto-ribosome, connecting the early RNA world to the protein world and forming the basis for the conservation of the ribosome in all three kingdoms of life (Davidovich et al., 2010).

\subsubsection{Structure and Function of the 70S Ribosome}

The translation of genetic information is responsible for $60 \%$ of the energy consumption and almost half the dry weight of a dividing cell (20,000 copies per cell) (Alberts et al., 2008). The prokaryotic ribosome forms a molecular machine of $2.5 \mathrm{MDa}$, composed of a large (50S) and a small (30S) subunit. The 30S subunit can be understood as the decoding center, meaning it is responsible for the fidelity of mRNA reading, whereas the $50 \mathrm{~S}$ subunit forms the peptidyl transferase center (PTC), where the peptide bond formation takes place. The 50S subunit contains a catalytic unit, which is composed of the P-region, the A-region and the A-loop of the 23S RNA (reviewed in Steitz, 2008; Schmeing and Ramakrishnan, 2009). The P-region and the A-region shape a symmetric entity, which is suggested to be the equivalent of the ancient proto-ribosome (Davidovich et al., 2010). Both, the 30S and the 50S subunits, contain three binding sites for tRNA: the A-site (aminoacyl), the P-site (peptidyl) and the E-site (exit). Compared to protein enzymes, which accelerate reactions up to $10^{23}$-fold, the ribosome, as all ribozymes, is relatively slow (acceleration rate of $10^{7}-10^{8}$ ). Moreover, different from enzymes, catalysis is based on entropic effects (Beringer et al., 2004). However, as tRNA accomodation, relevant for the fidelity of catalysis, is even slower, the ribozyme mechanism is evolutionary conserved. In Table 1.3, some properties of the $70 \mathrm{~S}$ ribosome are summarised (Rodnina, Marina V., Wintermeyer, Wolfgang, Green, 2011, Chapter 8, Table 1).

To understand the structural rearrangements of the active ribosome, the translation cycle will be briefly introduced according to Steitz (2008) and Schmeing and Ramakrishnan (2009). The translation can be divided into the following steps as illustrated in Figure (1.7): 
Table 1.3: Properties of the Prokaryotic 70S Ribosome

\begin{tabular}{|c|c|}
\hline Description & Properties \\
\hline Molecular mass & $2.5 \mathrm{MDa}$ \\
\hline Diameter & $\sim 275 \AA$ \\
\hline Sedimentation coefficent & $70 \mathrm{~S}$ \\
\hline RNA:protein ratio & $2: 1$ \\
\hline Relative density & $\sim 1.71 \mathrm{~g} / \mathrm{cm}^{3}$ \\
\hline Subunits & $30 \mathrm{~S}+50 \mathrm{~S}$ \\
\hline Small subunit composition & $16 \mathrm{~S} \mathrm{rRNA}(1542 \mathrm{nt})$ \\
\cline { 2 - 2 } & 21 proteins \\
\hline Large subunit composition & $23 \mathrm{~S} \mathrm{rRNA}(2904 \mathrm{nt})$ \\
\cline { 2 - 2 } & $5 \mathrm{~S} \mathrm{rRNA}(120 \mathrm{nt})$ \\
\cline { 2 - 2 } & 34 proteins \\
\hline Number of inter-subunit bridges & 13 \\
\hline Protein elongation rate & $4-22$ codons $s^{-1}$ at $37^{\circ} \mathrm{C}$ \\
\hline Error frequency & close to $10^{-3}$ \\
\hline Acceleration of peptide bond formation & $10^{7}-10^{8}$ \\
\hline
\end{tabular}

1. Initiation (reviewed in Sykes and Williamson, 2009):

The assembly of the 30S pre-initiation complex (30S PIC) is the starting point of the initiation. The IF3-bound 30S subunit recruits the mRNA, as well as IF1, IF2 GTP and fMet-tRNA ${ }^{\text {fmet }}$, the latter being the initiator tRNA. Interestingly, the mRNA is targeted first to the $30 \mathrm{~S}$ platform whereas the Shine-Dalgarno sequence binds the 3 ' end of $16 \mathrm{~S}$ rRNA. Upon reorganisation of the 30S PIC, the 30S initiation complex (30S IC) is assembled, where the mRNA is fully adapted into the mRNA channel and where A-site and P-site codons are ready for decoding. The fMet-tRNA ${ }^{\text {fmet }}$ has a preferred affinity to the P-site start codon due to the stabilisation by $16 \mathrm{~S}$ rRNA interactions. However, the detailed mechanism is not understood. The 70S initiation complex (70S IC) is formed by addition of the large ribosomal subunit (50S), resulting in GTP hydrolysation by IF2 and IF1 release. Once IF2 - GDP is released, the 70S IC can enter the elongation cycle.

2. Elongation (reviewed in Voorhees and Ramakrishnan (2013) and Rodnina, Marina V., Wintermeyer, Wolfgang, Green (2011, Section IV)):

The elongation cycle starts with the binding of the ternary complex composed by EF-Tu, GTP and aminoacyl-tRNA (aa-tRNA), to the 50S L7/L12 proteins. The cognate tRNA is recognised in the A-site by codon recognition, GTP hydrolysis and accommodation while EF-Tu is released. Cognate aa-tRNA interaction results in an optimal alignment after accommodation and peptidyl transfer from the P-site tRNA to the A-site tRNA in the peptidyl transferase center (PTC). The binding of EF-G is responsible for translocation by GTP hydrolysis. Translocation, the movement of both tRNA and mRNA relative to the ribosome from A-site to P-site (peptidyl-tRNA) and P-site to E-site (deacetylated tRNA), is the mandatory step 


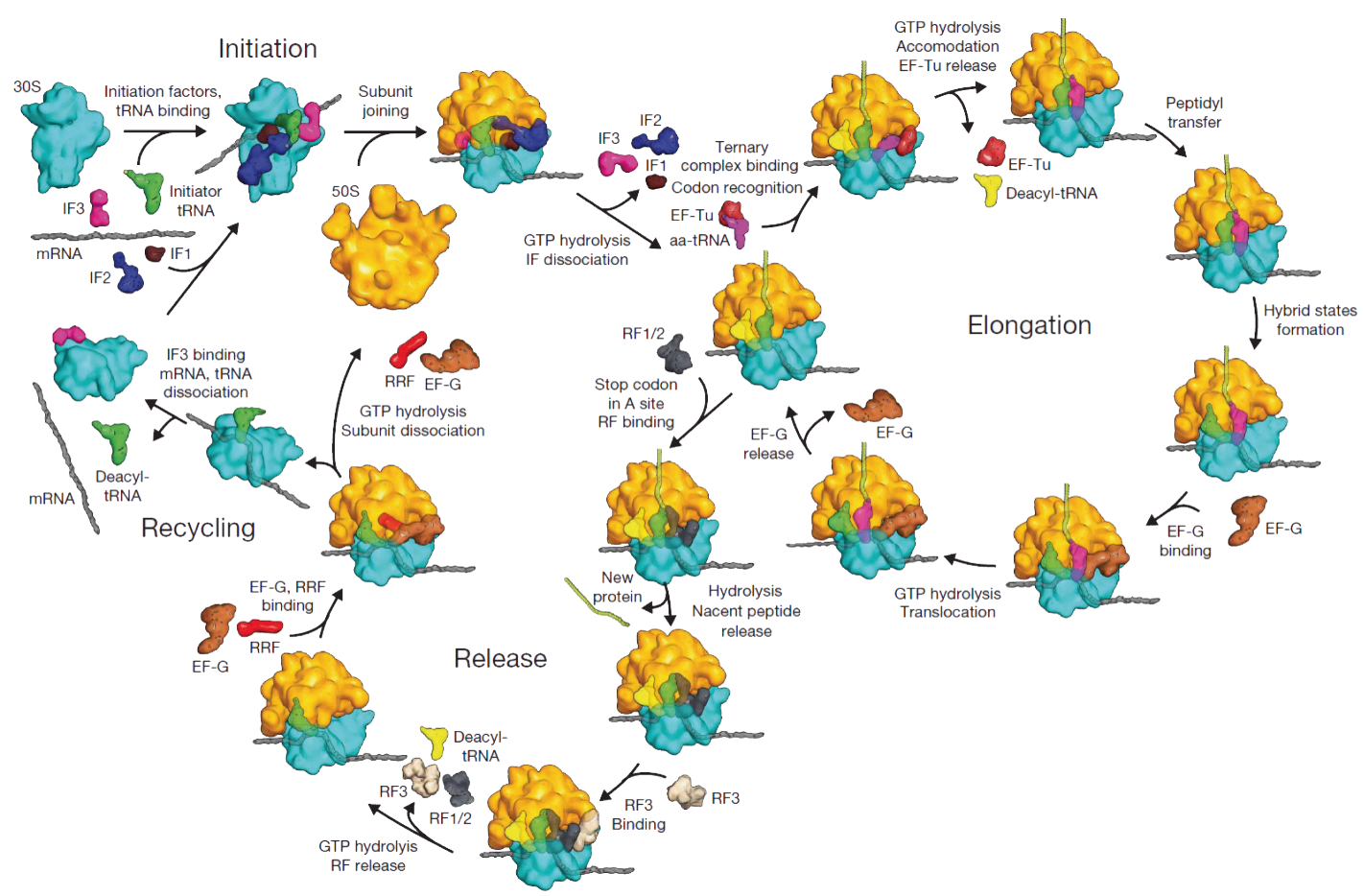

Figure 1.7: Overview of Prokaryotic Translation. The ribosomal translation cycle can be separated into initiation, elongation, release and recycling. Four GTPases are involved in the process: IF2 in initiation, EF-Tu in tRNA loading, EF-G in translocation and recycling and RF3 in the release of deacetylated tRNA. Further details can be found in the text (1.6.2). For simplicity, not all steps are shown here. This figure is reprinted with permission (Schmeing and Ramakrishnan, 2009).

to allow loading of another tRNA. Finally, EF-G is released and the ribosome can enter another elongation cycle until reaching a stop codon on the A-site.

3. Release (reviewed in Steitz, 2008):

When the stop codon (UUA or UAG) reaches the A-site of the ribosome, the elongation cycle is terminated. The binding of RF1/2 to the A-site triggers the release of the synthesised peptide chain from P-site tRNA. RF3, a GTPase, binds to $\mathrm{RF} 1 / 2$ and is relevant for the $\mathrm{RF} 1 / 2$ release. However, RF3 is not essential in bacteria and does not bind free $\mathrm{RF} 1 / 2$.

4. Recycling (reviewed in Pai et al. (2008) and Rodnina, Marina V., Wintermeyer, Wolfgang, Green (2011, Chapter 14)):

EF-G and the ribosome release factor (RRF) can bind to the post-termination ribosome. Interestingly, EF-G is shown to play a double role, relevant for translocation and for recycling. The post-termination ribosome consists only of the small subunit, the big subunit and a deacetylated tRNA in the P-site. Rapid GTP hydrolysis results in conformational changes in the $30 \mathrm{~S}$ subunit. After Pi is released, EF-G undergoes a conformational change and the 50S subunit disassembles from the $30 \mathrm{~S}$ subunit. IF3 promotes the dissociation of tRNA and mRNA from 30S subunit in order to be ready for another round of initiation. 
This work is mainly related to elongation, specifically to the translocation step of elongation. Therefore, initiation release and recycling will not be explained in greater detail in this work.

In the following section, the elongation cycle will be illustrated in greater detail:

Two GTPases are involved in the elongation cycle. EF-Tu is relevant for the delivery of cognate aa-tRNA into the ribosomal A-site, whereas, after peptidyl transfer, EF-G facilitates translocation. The ability of the ribosome to distinguish between cognate, nearcognate and non-cognate tRNA is the prerequisite for a functional protein polymerase, with an overall error frequency ${ }^{21}$ of $10^{-3}$ to $10^{-5}$ (Wohlgemuth et al. (2010) and reviewed in Allan Drummond and Wilke (2009)). L7/12 are required for the binding of translation factors. Multiple copies of L7/12 in E. coli bind EF-Tu - GTP, and codon reading is facilitated in the $30 \mathrm{~S}$ subunit between the tRNA anticodon stemloop and the mRNA codon. In case of cognate pairing of the anticodon-codon complex, A-1492, A1493 and G-530 of the 16S rRNA are repositioned (reviewed in Ogle and Ramakrishnan, 2005; Steitz, 2008; Schmeing and Ramakrishnan, 2009). These changes are accompanied by a repositioning of the $30 \mathrm{~S}$ head domain towards the $50 \mathrm{~S}$ subunit, resulting in the stimulation of EF-Tu GTP hydrolysis. The correct coding, however, needs to be signalled over $80 \AA$, which entails tRNA deformation (Stark et al., 1997, 2002; Fischer et al., 2015) and EF$\mathrm{Tu} \cdot$ sarcin-ricin loop (SRL) interaction. Interestingly, paromomycin binding triggers the same effect in combination with near-cognate tRNAs and thus induces misincorporation errors (Carter et al., 2000). After GTP hydrolysis and Pi dissociation, the cognate aa-tRNA is accommodated, meaning that the CCA tail is repositioned into the PTC on the 50S subunit. The fidelity of decoding is controlled in a two-step process, the initial selection and thereafter the proof reading of the delivered aa-tRNA. The peptide bond formation is accomplished by a proton shuttle mechanism after nucleophilic attack of $\alpha-\mathrm{NH}_{2}$, where the 2'-OH group of the A-site A76 ribose plays a main role (Rodnina et al., 2007). In summary, the mechanism of the acceleration of this reaction is based on the decrease of activation entropy by positioning the two substrates, ordering water molecules within the active site and providing an electrostatic network for an intermediate state formation (Beringer and Rodnina, 2007). It was shown that, spontaneously, hybrid states can form, where the CCA ends are positioned into the P- and the E-site on the 30S subunit (Moazed and Noller, 1989b) ((P/E) Joseph and Noller, 1998; McGarry et al., 2005; Walker et al., 2008) ((A/P) Semenkov et al., 2000; McGarry et al., 2005; Dorner et al., 2006).

The next step of elongation is the EF-G-triggered translocation. EF-G, a member of the GTPase superfamily, is composed of five domains (Czworkowski et al., 1994). The

\footnotetext{
${ }^{21}$ The process of cognate tRNA recognition is denoted "decoding". Premature chain termination accounts for a ten-fold increase of the value given in Table 1.3, which describes only initial selection and proof-reading.
} 
N-terminal domain 1, which is the GDP/GTP binding G domain, and domain 2 have similarities to the corresponding domains in EF-Tu, while there is no counterpart for the three remaining domains. The domain 4 of ribosome-bound EFG reaches into the decoding center (Stark et al., 2000; Gao et al., 2009). Translocation is the coupled movement of the ASL of two adjacent tRNAs and the mRNA on the 30S subunit from A and $\mathrm{P}$ to $\mathrm{P}$ and $\mathrm{E}$, respectively. This translocation is coupled with the reversal of the socalled "ratcheting", the rotation of the 30S body relative to the 50 S subunit (Frank and Agrawal, 2000). Accordingly, the dynamics can be understood as a tRNA movement relatively to the $30 \mathrm{~S}$ subunit, coupled with a discrete and reversing $30 \mathrm{~S}$ subunit movement relative to the 50S subunit. Although in vivo this process is assisted by the GTPase EF-G, in vitro, translocation can happen spontaneously but very slowly (0.0002-0.02 $s^{-1}$ ) without the addition of EF-G, and it is driven by the tRNAs' affinities (Shoji et al., 2006; Konevega et al., 2007). Interesting in this context is that, in the absence of EF-G, the ribosome undergoes slow and spontaneous movements in both direction, so-called forward-translocation and retro-translocation. The directionality depends on the specific tRNA affinities. For some tRNA combinations, spontaneous backward-translocation is preferred (Konevega et al., 2007). Most likely, EF-G accelerates pre5 to post1 transition, which was shown to be rate limiting in retro-translocation (Fischer et al., 2010). EF-G may stabilise pre-existing ribosome conformations and induce novel transient states that allow the detachment of the tRNA-mRNA complex from the 30S subunit and prevent backward movement (Fischer et al., 2010). The main acceleration depends on GTP hydrolysis, as shown with non-hydrolysable analogues of GTP and a GTP-hydrolysis-insufficient mutation (H91A) (Rodnina et al., 1997; Holtkamp et al., 2014). Domain 4 of EF-G was shown to be important for directing the forward movement of peptidyl-tRNA by occupying the A-site. $\mathrm{P}_{i}$ release seems to be relevant only for the obviation of premature EF-G release. After $\mathrm{P}_{i}$ release, $\mathrm{EF}-\mathrm{G}$ can dissociate.

\subsubsection{Dynamics of Translocation}

In the so-called classical state, the anti-codon-stem loop (ASL) remains in the same position as the acceptor ends, expressed with the term $\mathrm{A} / \mathrm{A}, \mathrm{P} / \mathrm{P}$ or $\mathrm{E} / \mathrm{E}$, respectively. While the tRNA performs large distance movements, several intermediate states exist. In one of these states, the so-called tRNA hybrid state, the ASL remains bound to the Aand P-site of the 30S subunit, whereas the acceptor ends of both tRNAs are positioned in the adjacent $\mathrm{P}$ - and $\mathrm{E}$-site of the $50 \mathrm{~S}$ subunit (A/P and $\mathrm{P} / \mathrm{E}$ ) (Moazed and Noller, 1989b). The role of these states was greatly studied in several publications related to P/E state (Joseph and Noller, 1998; McGarry et al., 2005; Walker et al., 2008) and A/P state (Semenkov et al., 2000; McGarry et al., 2005; Dorner et al., 2006). These 
a b

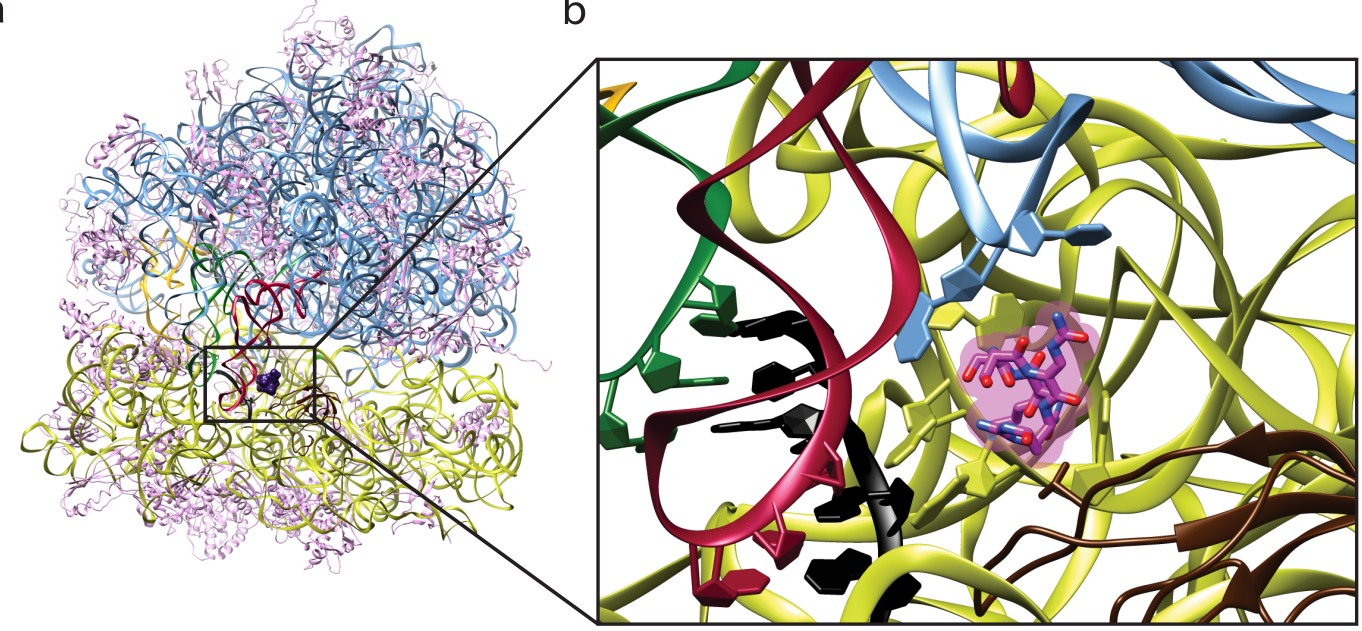

C

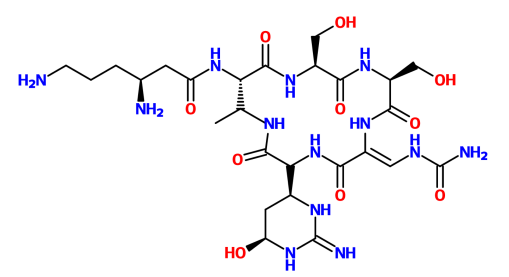

d

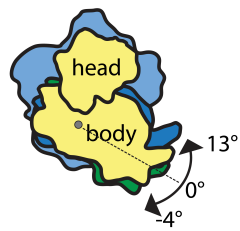

$30 \mathrm{~S}$ body rotation

e

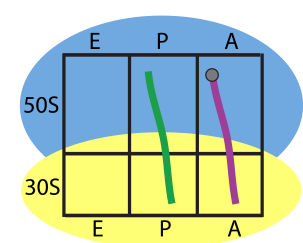

classical (A/A \&P/P)

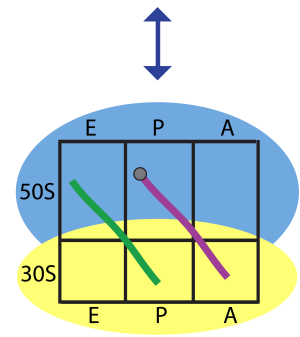

hybrid $(A / P \& P / E)$

Figure 1.8: Viomycin Effect on Translocation. a: The crystal structure of the viomycin-bound $70 \mathrm{~S}$ ribosome shows the antibiotic at the interface between helix 44 of the 30S subunit (yellow) and helix 69 of the 50S subunit (blue) in proximity to the A-site tRNA (red). b: In the close-up, the details are illustrated. S12 (brown) is the only protein close to viomycin. Shown are: Viomycin as space-filling model in dark purple in the overview (a) or as stick model (highlighted in purple) in the close-up view (b); mRNA in black; A-site tRNA in red; P-site tRNA in green; E-site tRNA in deep yellow; 50S subunit in light blue; 30S subunit in light yellow; S12 in brown; all other proteins in light purple (a) or removed (b). The codon-anticodon interactions between tRNA and mRNA are shown in the close-up view (according to Stanley et al., 2010). c: Structural formula of tuberactinomycin-family antibiotic viomycin (prepared with www.emolecules.com). d: Translocation is known to be coupled to 30S dynamics. Here, 30S body rotation around helix $27(\mathrm{~h} 27)$ of $16 \mathrm{~S}$ rRNA as pivot point is represented schematically (according to Fischer et al., 2010). e: During translocation, tRNAs undergo a movement from A-site to P-site and from P-to E-site. Due to asynchronous movement of the acceptor arm (50S) and the anticodon arm (30S), intermediate states, so-called hybrid states, are formed. Exemplary, the $\mathrm{P} / \mathrm{E} \& \mathrm{~A} / \mathrm{P}$ hybrid state is shown with the classical A/A \& P/P state. f: Plotted are the $30 \mathrm{~S}$ dynamics (body rotation and head dynamics) relative to the tRNA dynamics. Although the coupling is loose, a joint movement of tRNAs and 30S subunit is detectable. Schemes d-f adapted with permission (Fischer et al., 2010). 
states correlate with a rotational state of the $30 \mathrm{~S}$ body, with the corresponding rotation being termed ratcheting (Frank and Agrawal, 2000). The importance of the ratcheting is highlighted by the fact that intersubunit crosslinking between $30 \mathrm{~S}$ and 50S, which abolishes ratcheting, results also in the inhibition of translocation (Horan and Noller, 2007). Even more, smFRET experiments observed that the EF-G-free pre-translocation complex consists of a dynamic equilibrium between tRNA hybrid and tRNA classical states (Blanchard et al., 2004; Kim et al., 2007; Munro et al., 2015). In addition to these hybrid states, further intermediates have been identified. A trajectory of spontaneous tRNA movement was shown by time-resolved cryo EM. 50 different tRNA states were defined by an unbiased computational sorting of 2,000,000 ribosome particles (Fischer et al., 2010). This permitted a quasi-continuous trajectory classified into eight most distinct states, revealing classical, hybrid and novel intermediate states. Very interesting is the local and global coupling of tRNA dynamics and ribosome dynamics. The rotational movement (between $14^{\circ}$ and $-6^{\circ}$ ) is accomplished by a 30S head tilting (between $-1^{\circ}$ and $4^{\circ}$ ) around the helice 28 as pivot point. Even more, a perpendicular so-called head swivelling motion is recognised. Locally, movements of helices 38 and 69 of 23S rRNA, protein L5, L16 and L1 stalk convoy the tRNA movement over a distance of $8 \AA$ to $40 \AA$. As the ratio of particles in a certain state does not change over time, they are in rapid equilibrium, while the transition from pre5 to post1 is very slow. This is consistent with previous data (Shoji et al., 2006; Konevega et al., 2007). The probability of high degree 30S body rotations increase with hybrid state formation, and this rotation is reversed by pre-to-post transition. Interestingly, the coupling of tRNA movement and 30S subunit dynamics is loose, as the calculated energy landscape is very flat. This explains why there are several different ribosome conformations found for each tRNA state, with the pre2 to pre3 transition forming a bottleneck compared to other pre state transitions. Even more, ribosome dynamics increase with rising temperature $\left(4^{\circ} \mathrm{C}, 18^{\circ} \mathrm{C}, 37^{\circ} \mathrm{C}\right)$, meaning that, at elevated temperature, the thermal energy is sufficient to allow every possible 30S body rotation with high probability. In a more recent and related work (Bock et al., 2013), 13 different intermediate-translocation-state models were obtained. This was done by refining cryo EM models against atomistic crystal structure models and subjecting them to MD simulation. This allowed a pseudo-atomistic description of transition states-related forces and energy barriers. It was shown that tRNA, moving from $\mathrm{A} / \mathrm{A}$ to $\mathrm{A} / \mathrm{P}$ state, is stabilised by two different patches (patch 1 or 2, respectively) of L16. L5 maintains a flexible contact, sliding over the tRNA moving from $\mathrm{P} / \mathrm{P}$ to $\mathrm{P} / \mathrm{E}$ site. It was shown that L1 stalk connects inter-subunit rotation to translocation from the P- to the E-site. High intersubunit rotation (pre3) stabilises the closed L1 stalk through S7, while after back-rotation (post1) the interaction is lost and L1 can pull the tRNA into the E-site. It was shown that $30 \mathrm{~S}$ body rotation can happen faster than the tRNA transition. 


\subsubsection{Ribosome-targeting Antibiotics}

Many early classes of antibiotics used in clinical practice target bacterial protein synthesis, as chloramphenicol and tetracycline (both discovered in 1949), aminogycosides and macrolides (discovered in 1950 and 1952, respectively). Antibiotics, used in structural and kinetic studies, contributed to the understanding of functional steps in protein synthesis, for example in case of the decoding (Carter et al., 2000) and the peptidyl transfer (Hansen et al., 2002). Especially, as certain steps of protein synthesis are stalled, ribosomes in complex with antibiotics were used for cryo EM (Stark et al., 2000) and X-ray analysis (Gao et al., 2009). Even more, the mechanism of several antibiotics have been elucidated. In the last 15 years, the binding site of at least one member of the most relevant antibiotics classes has been defined, with most antibiotics shown to act close to the 30S decoding center, like aminoglycosides, streptomycin, tetracycline or spectinomycin, or to the 50S peptidyl transferase center, like macrolides and ketolides, chloramphenicol or puromycin (reviewed in Wilson, 2009; Blanchard et al., 2010). An interesting example for the action on the 30S decoding center is the aminoglycoside paromomycin. Paromomycin, binding to helix 44 on the $30 \mathrm{~S}$ subunit, inhibits translocation by stabilising the A-site attachment (Peske et al., 2004) for both cognate and near cognate tRNAs. Additionally, it enhanced aminoacid misincorporation by stabilising near-cognate tRNAs, as was shown before for other aminoglycosides. This is accomplished by the mimicry of the cognate codonanticodon interaction, as 16S rRNA A1492 and A1493 are displaced and are pointing into the A-site (Carter et al., 2000). However, the strength of the inhibition on translocation was discussed controversially (160-fold: Peske et al., 2004) (4-fold - 6-fold: Feldman et al., 2010). An example of an antibiotic acting on the peptidyl transferase center is puromycin. Puromycin structually mimics the terminal aminoacyl-adenosine moiety of aa-ttRNA, thereby functioning as an acceptor in the peptide bond formation so that the nascent peptide is released bound to puromycin (reviewed in Blanchard et al., 2010). Only few antibiotics show different binding sites like orthosomycin (L16, H89, H91) (Mikolajka et al., 2011) or fusidic acid (EF-G between domain III and the G-domain) (Chen et al., 2010a). Beside structural studies, fluorescence (FRET) and fast-kinetic approaches (stop-flow, quench flow) revealed inhibitory mechanism like in the case of translocation inhibitions (Peske et al., 2004). Single-molecule FRET approaches helped to deepen the knowledge of inhibitory effects like in the case of translocation, inducing peptidyl transferase A-site binding antibiotics (Ermolenko et al., 2013) or aminoglycoside activity on ribosomal pre-translocation complexes (Feldman et al., 2010). Using several kinetic and structural approaches with antibiotics, the ribosome peptide transferase mechanism was elucidated (Beringer and Rodnina, 2007). 
However, in some cases the inhibitory effect of antibiotics is discussed very controversially, and it is often limited depending on the technique used to study the small molecule, as in the case of viomycin. Viomycin is a member of the tuberactinomycin family, synthesised modularly by non-ribosomal peptide synthetases (NRPS) of Streptomyces strains (Yin et al., 2003; Thomas et al., 2003; Barkei et al., 2009). It was used initially as a drug against tuberculosis until it was replaced by the less toxic capreomycin. Viomycin was shown to stabilise the peptidyl-tRNA in the A-site more than 1,000-fold and to inhibit translocation more than 10,000-fold (Peske et al., 2004). An X-ray structure defined the binding site of viomycin at the interface between helix 44 of the small subunit, with contacts to the anticodon stem of A-site tRNA and helix 69, with contacts to both A- and P-site tRNA. Viomycin is in close proximity to A1492, A1493 and G1491 of h44 and to A1913 and C1914 of H69 (Stanley et al., 2010). Also, it influences the position of A1492, A1493 and A1913. Interestingly, there is a great overlap of interactions with aminoglycosides, especially paromomycin (reviewed in Blanchard et al., 2010). Earlier, the binding site was discussed controversially, especially as two different and independent binding sites (H69 and h44) were proposed based on viomycin influenced mutational resistance and methylation studies (Yamada and Bierhaus, 1978; Moazed and Noller, 1987; Maus et al., 2005; Johansen et al., 2006). In the crystal structure of viomycin, the ribosome is present in a non-rotated state and tRNAs are bound in the classical A-,P- and E-site (Stanley et al., 2010). Different from that, FRET experiments suggest a rotated state of the 30S subunit (Ermolenko et al., 2007; Cornish et al., 2008), while they showed, by chemical footprinting, interaction correlated with the $\mathrm{A} / \mathrm{P} \& \mathrm{P} / \mathrm{E}$ hybrid state formation. Based on FRET assays it was shown that viomycin suppresses tRNA fluctuations between the classical and the hybrid state by prolonging the life time of each state (Kim et al., 2007). In two studies, viomycin was shown to stabilise the A-site tRNA (Peske et al., 2004; Pan et al., 2007), whereas the latter study suggests that, after the addition of EF-G and GTP, $\mathrm{A} / \mathrm{A} \& \mathrm{P} / \mathrm{E}$ hybrid state formation is possible, but $\mathrm{A} / \mathrm{P} \& \mathrm{P} / \mathrm{E}$ hybrid state formation is prevented. Different ionic conditions as well as different experimental set-ups may account for the controversial results. It seems likely that A-site tRNA is stabilised. However, it remains unclear if a classical or a hybrid state, and a non-rotated or a rotated state are preferred. 


\subsection{Aim of This Work}

This work can be divided into a part related to the structural elucidation of cohesin and a part related to the analysis of the effect of viomycin on $70 \mathrm{~S}$ ribosome dynamics.

Cohesin, a key regulator of sister chromatid cohesion, is mandatory for maintaining the topological arrangement of chromatin, while especially mitosis and meiosis require the correct and equal allocation of sister chromatids into the daughter cells. Cohesin is known to form a ring-like structure, which is likely to establish sister chromatid cohesion by entrapment. As outlined in Section 1.5.6, X-ray structures of individual cohesin subunits and domains are solved. Structural models of the Smc1/3 hinge, the Smc1/3 NBDs in interaction with N-terminal (Smc3) or C-terminal (Smc1) parts of Scc1 have been obtained. Besides, a structure of SA2 in interaction with a fragment of Scc1 was solved. However, a complete cohesin structural model has not been described and the exact topology of cohesin is not known. This work aims to provide the first topological description of cohesin by combining single particle EM results with the available X-ray structures. Pds5 and Wapl are known to be regulative interaction partners of cohesin. Their biological role in the modulation of cohesin opening with respect to the prophase pathway is known but not yet well understood. Their structure and binding site on cohesin is unknown. Accordingly, these cohesin-bound proteins are very interesting targets for a structural study by single particle electron microscopy. In this work, it is aimed to study Pds5- and Wapl-bound cohesin by single particle EM and to define their binding sites.

The strong inhibitory effect of viomycin on translocation in the 70S ribosome and the viomycin binding site are known. However, as depicted in Section 1.6.4, the concrete inhibitory mechanism of viomycin is discussed controversially. It is assumed that viomycin binding effects the 30S body rotation and the tRNA dynamics. In this work, the effect of viomycin on the 30S body rotation and on the tRNA dynamics is aimed to be disentangled. Using single particle electron cryo-microscopy, many open question regarding the influence of viomycin on ribosome dynamics can be addressed. Moreover, this approach aims to be understood as a proof of principle that single particle electron cryo-microscopy can be a structural approach to investigate pharmacological questions. 


\section{Chapter 2}

\section{Materials and Methods}

There is need of a method for finding out the truth.

— René Descartes

\subsection{Biochemical Approach}

The cloning, expression and purification of cohesin were performed in Prof. Jan-Michael Peters lab at IMP Vienna by Dr. Pim Huis in 't Veld. Moreover, the ATPase assays, the ProteoPlex assay (Haselbach, 2014) and low angle Pt/C rotary shadowing were performed by Dr. Pim Huis in 't Veld. Samples were freshly prepared at IMP or in Göttingen and instantly used for single particle EM purposes.

$70 \mathrm{~S}$ ribosomes were purified in Prof. Marina Rodnina's lab at MPI for Biophysical Chemistry by Dr. Frank Peske and colleagues. Stop-flow experiments for 70 S ribosome dissociation tests were performed by Dr. Frank Peske.

\subsubsection{Purification of Bonsai Cohesin}

The purification of cohesin complex and bonsai cohesin complex will be briefly summarised. The detailed biochemical approach is reviewed in "Reconstitution of the human cohesin complex" by Pim Huis in 't Veld (Huis in 't Veld, 2013).

To express human Smc1 $1^{\text {bonsai }}$, Smc $3^{\text {bonsai }}$, Scc1 and SA1, a Sf9 cell line of the fall armyworm Spodoptera frugiperda was infected using a multi-bac system, encoding each of the corresponding subunits in equal stoichiometry. Pds5 and Pds5-Wapl were purified independently. By addition of Pds5 or Pds5-Wapl, during the tandem protein purification step of bonsai cohesin tetramer (Smc1 ${ }^{\text {bonsai }}$, Smc3 $3^{\text {bonsai }}$, Scc1 and SA1), Pds5 - bonsai cohesin tetramer and Wapl · Pds5 - bonsai cohesin tetramer was obtained. Similarly, the addition of Pds5 to bonsai cohesin trimer (Smc1 ${ }^{\text {bonsai }}$, Smc3 $3^{\text {bonsai }}$ and Scc1) yielded Pds5 - bonsai cohesin trimer. A complete list of all cohesin complexes and containing 
subunits used in this work is given in Table 3.1. Throughout this work, "cohesin" is used to refer to the "bonsai cohesin" with the shortened bonsai Smc1/3 versions, while the term "full-length cohesin" is used for the unmodified cohesin.

Cloning was performed using standard restriction-ligation methods and amplification was accomplished with high fidelity Phusion polymerase. A fragment containing Smc3 and SA1 was inserted into a vector containing Scc1 and Smc1. Plasmids were transformed into competent DH5 $\alpha$ E. coli and purified by mini-prep (Quiagen) or midi-prep (Promega). DH10Bac E. coli bearing a modified version of a $136 \mathrm{~kb}$ viral bacmid bMON14272 (Invitrogen) were used for the transformation of the $19.6 \mathrm{kbp} 4$-gene transfer vector. The recognition sequences were the Tn7L and the Tn7R locus. Bacmids, positively tested for the transposed plasmid, were extracted and precipitated using isopropanol precipitation. Washed and resuspended DNA was transfected into adherent Sf9 insect cells. The initial virus was harvested 60-72 hours after transfection and was used to infect a Sf9 suspension culture. Successful expression was monitored by measuring YFP expression and Sf9 cells were harvested by centrifugation. Resuspended and repelleted cells were frozen in liquid nitrogen and stored at $-80^{\circ} \mathrm{C}$. After standard lysis and lysate clearing, a tandem protein purification approach was chosen for the cohesin complex. First, a metal-chelate affinity purification step (Ni-NTA) was performed with a polyhistidine-tag placed N-terminally in SA1. The pooled eluate was used for a second purification step. With Anti-FLAGM2-Agarose beads (Sigma), the complex was attached by the C-terminal FLAG tag of Smc3. After washing, the complex was either directly eluted or Pds5 or Pds5-Wapl, respectively, were added to the attached complex. Eventually, elution was performed with FLAG peptide, whereby ATP $\gamma \mathrm{S}$ (Jena Bioscience) at a concentration of $0.1 \mathrm{mg} / \mathrm{mL}$ was added. The tandem Ni-NTA-FLAG purification yielded pure and stoichiometric protein complexes for single particle EM and was used for a subsequent GraFix run.

In principle, cohesin and bonsai cohesin were prepared similarly. However, the huge flexibility of full length cohesin was shown to be unfavourable for single particle EM. It was decided to remove the most flexible coiled-coils as shown for Ndc80 complex (Ciferri et al., 2008). Prediction programs allowed to analyse cohesin subunits and predict secondary structures (Rost et al., 2004). Paircoil2 and COILS/PCOILS was used to predict the localisation of Smc1/3 coiled-coil regions (McDonnell et al., 2006; Lupas et al., 1991; Lupas, 1996). Accordingly, most of the Smc1 and Smc3 coiled-coils were removed, while the predicted coiled-coil twist of the ligated ends in bonsai cohesin was preserved.

In a ProteoPlex assay, the addition of $\mathrm{ATP} \gamma \mathrm{S}$ was identified to stabilise bonsai cohesin complex (Haselbach, 2014). The identified most stable buffer conditions, were used for all cohesin complexes in this work. The elution buffer contained $3 \mathrm{mg} / \mathrm{ml}$ FLAG peptide in $25 \mathrm{mM}$ HEPES pH 7.5, $150 \mathrm{mM} \mathrm{NaCl}, 2 \mathrm{mM} \mathrm{MgCl}_{2}$ and $5 \%$ glycerol. 


\subsubsection{Purification of 70S Ribosome}

The details of $70 \mathrm{~S}$ ribosome purification will not be explained here but can be reviewed in Konevega et al. (2007). The main components of the ternary complex (TC), the aminoacyl-tRNA $\left(\left[{ }^{14} \mathrm{C}\right]\right.$ Phe-tRNA $\left.{ }^{\text {Phe }}\right)$, GTP and EF-Tu were prepared according to Table 2.1. To remove EF-Tu-bound GDP, pyruvate kinase (PK) and phosphoenolpyruvate (PEP) were added in excess. Thus, EF-Tu was prevented from binding GDP instead of GTP. Accordingly, the Phe-tRNA ${ }^{\text {Phe }}$ was hindered from hydrolysis by EF-Tu (GTP) binding. The initiation complex (IC) is composed of the $30 \mathrm{~S}$ subunit, the $50 \mathrm{~S}$ subunit, the mRNA, the N-formylmethionine and the tRNA (fMet-tRNA ${ }^{f M e t}$ ) in the P site. The composition of the IC sample is depicted in Table 2.2. A gel-filtration matrix (BioSuite ${ }^{T} M$ Column) was used for purification of the initiation complex (IC), avoiding contaminations of the gradient forming sugars, which are contrast-limiting in electron micrographs. Additionally, this procedure removed the initiation factors (IFs). After purification, the initiation complex (IC) was snap-frozen in liquid nitrogen and was stored at $-80^{\circ} \mathrm{C}$. The ternary complex (TC) and the IC were mixed immediately before vitrification and incubated for $30 \mathrm{~s}$ at room temperature to form the pre-translocation complex (pre-TC) ${ }^{1}$. In Table 2.3, the composition of the mixed solution is shown. The formed pre-TC was loaded with two tRNAs, one in the $\mathrm{A} / \mathrm{A}$ site and the other in the $\mathrm{P} / \mathrm{P}$ site. Although spontaneous translocation can happen, the reaction rate is very slow without EF-G. Cryo sample preparation was accomplished within the first 30 minutes after pre-TC formation.

The composition of the used buffers is defined in Table 2.3, with equivalent HAKM buffers ${ }^{2}$ for TC and IC buffers. The $M g^{2+}$ concentration has a strong influence on the $70 \mathrm{~S}$ ribosome stability. At low $\mathrm{Mg}^{2+}$ concentrations $(3.5 \mathrm{mM})$, the $70 \mathrm{~S}$ ribosome is less stable and can disassemble within minutes, while, at higher concentrations (15 $\left.\mathrm{mM} \mathrm{Mg}^{2+}\right)$, the stability is increased, but the tRNA binding accuracy is reduced (personal communication with Dr. Frank Peske). However, satisfying conditions can be reached by the addition of polyamines. Polyamines, small poly-cationic molecules, were shown to interact strongly with RNA and to positively influence the stability of the $70 \mathrm{~S}$ ribosome (Figure 2.1b).

To define stabilzing buffer conditions light scattering experiments were performed. It was shown that the addition of polyamines can reduce the dissociation of the 70S ribosome into $30 \mathrm{~S}$ and $50 \mathrm{~S}$ subunits (Figure $2.1 \mathrm{a})$. $70 \mathrm{~S}$ ribosomes $(0.25 \mu \mathrm{M})$ were mixed in a stop-flow apparatus with IF3 $(2 \mu \mathrm{M})$, an anti-reassembly factor. Two different $M g^{2+}$ concentrations (3.5 mM or $15 \mathrm{mM}$ ) were tested at $436 \mathrm{~nm}$ light. $70 \mathrm{~S}$ ribosome disassembly was reduced by addition $0.5 \mu \mathrm{M}$ spermidine and $0.5 \mu \mathrm{M}$ putrescine. Further increase of putrescine concentration $(8 \mu \mathrm{M})$ does not improve the $70 \mathrm{~S}$ ribosome stability. The strongest tested

\footnotetext{
${ }^{1}$ The formation of the pre-translocation complex (pre-TC) is an extremely fast reaction.

${ }^{2} \mathrm{HAKM}$ for $\mathbf{H E P E S}$, ammonium chloride, $\mathbf{K}$ for $\mathrm{KCl}$ and magnesium chloride
} 
stabilisation effect was shown for a combination of relatively low concentrations of spermine $(0.5 \mu \mathrm{M})$ and spermidin $(0.4 \mu \mathrm{M})$. Apparently, spermine shows the strongest influence. However, the increased $\mathrm{Mg}^{2+}$ concentration $(7 \mathrm{mM})$ enhanced the stability as well. It seems as if the stability is related to a combination of the chain length and the basic character (the number of positive charges) of the specific polyamine (Figure 2.1b). While spermine, the tested polyamine possessing the longest chain, shows the strongest effect in stabilisation, it was decided to use a combination of putrescine and spermidine for cryo EM, as these are the naturally occurring polyamines in E. coli.

Table 2.1: Preparation Condition of Ternary Complex (TC). The ternary complex (TC) is composed of Phe-tRNA ${ }^{\text {Phe }}, \mathrm{EF}-\mathrm{Tu}$ and GTP. The mixture was incubated for $15 \mathrm{~min}$ at $37^{\circ} \mathrm{C}$.

\begin{tabular}{|c|c|}
\hline Additive & Concentration \\
\hline HEPES pH 7.5 & $50 \mathrm{mM}$ \\
\hline Ammonium chloride $\left(\mathrm{NH}_{4} \mathrm{Cl}\right)$ & $70 \mathrm{mM}$ \\
\hline Potassium chloride $(\mathrm{KCl})$ & $30 \mathrm{mM}$ \\
\hline Magnesium chloride $\left(\mathrm{MgCl}_{2}\right)$ & $7 \mathrm{mM}$ \\
\hline GTP & $1 \mathrm{mM}$ \\
\hline PEP & $3 \mathrm{mM}$ \\
\hline Pyruvate kinase & $100 \mu \mathrm{g} / \mathrm{ml}$ \\
\hline EF-Tu (GDP) & $2.4 \mu \mathrm{M}$ \\
\hline$\left[{ }^{14} \mathrm{C}\right]$ Phe-tRNA ${ }^{\text {Phe }}$ & $1.2 \mu \mathrm{M}$ \\
\hline
\end{tabular}

Table 2.2: Preparation Condition of Initiation Complex (IC). The mixture was incubated for 30 min at $37^{\circ} \mathrm{C}$. A gel-filtration matrix (BioSuite ${ }^{T M}$ Column) was used for purification.

\begin{tabular}{|l|l|}
\hline Additive & Concentration \\
\hline HEPES pH 7.5 & $50 \mathrm{mM}$ \\
\hline Ammonium chloride $\left(\mathrm{NH}_{4} \mathrm{Cl}\right)$ & $70 \mathrm{mM}$ \\
\hline Potassium chloride $(\mathrm{KCl})$ & $30 \mathrm{mM}$ \\
\hline Magnesium chloride $\left(\mathrm{MgCl}_{2}\right)$ & $7 \mathrm{mM}$ \\
\hline GTP & $1 \mathrm{mM}$ \\
\hline 70S ribosome & $0.6 \mu \mathrm{M}$ \\
\hline mRNA & $1.8 \mu \mathrm{M}$ \\
\hline IF1 & $0.9 \mu \mathrm{M}$ \\
\hline IF2 & $0.9 \mu \mathrm{M}$ \\
\hline IF3 & $0.9 \mu \mathrm{M}$ \\
\hline fMet-tRNA & $1.8 \mu \mathrm{M}$ \\
\hline
\end{tabular}

Table 2.3: Final Mixture of Initiation Complex (IC) and Ternary Complex (TC). Pretranslocation complexes (pre-TC) are formed.

\begin{tabular}{|l|l|}
\hline Description & Properties \\
\hline HEPES pH 7.5 & $50 \mathrm{mM}$ \\
\hline Ammonium chloride $\left(\mathrm{NH}_{4} \mathrm{Cl}\right)$ & $70 \mathrm{mM}$ \\
\hline Potassium chloride $(\mathrm{KCl})$ & $30 \mathrm{mM}$ \\
\hline Magnesium chloride $\left(\mathrm{MgCl} \mathrm{C}_{2}\right)$ & $3.5 \mathrm{mM}$ or $15 \mathrm{mM}$ \\
\hline Spermidine & $0.5 \mathrm{mM}$ \\
\hline Putrescine & $0.5 \mathrm{mM}$ \\
\hline Viomycin & non or $200 \mu \mathrm{M}$ \\
\hline IC & $0.035 \mu \mathrm{M}$ \\
\hline TC & $0.07 \mu \mathrm{M}$ \\
\hline
\end{tabular}



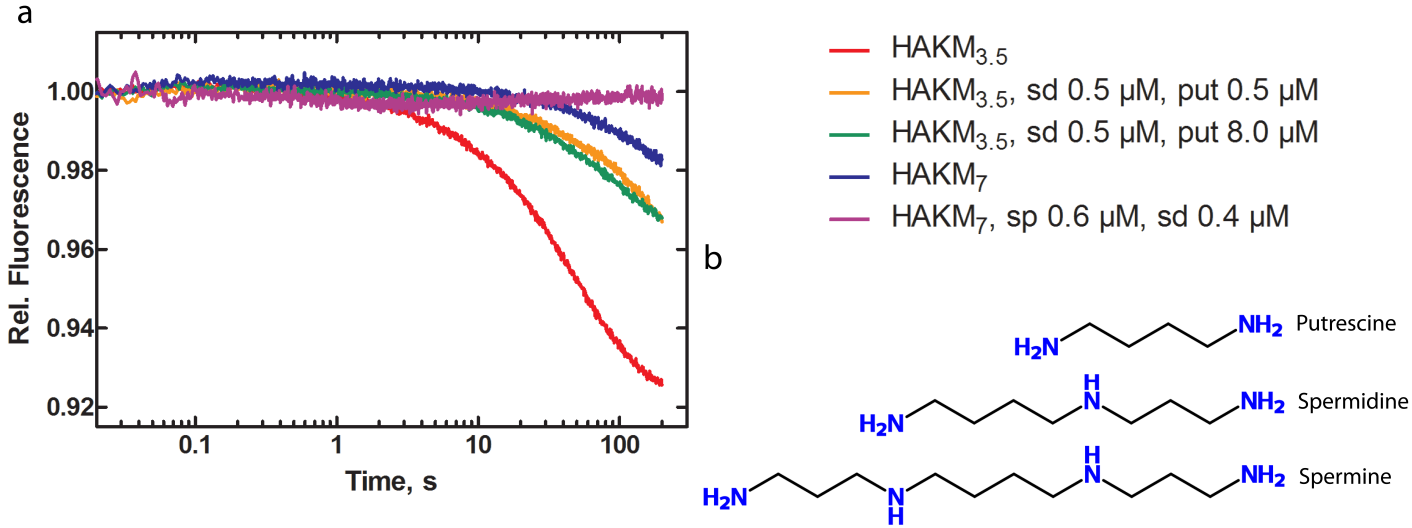

Figure 2.1: 70S Ribosome Dissociation Monitored by Light Scattering. a: Light scattering of $0.25 \mathrm{\mu M} 70 \mathrm{~S}$ ribosome at $37^{\circ} \mathrm{C}$ with different polyamine compositions was measured in a stop-flow system. The experiments were conducted at $436 \mathrm{~nm}$. Reassembly is prevented by addition of IF3 at $2 \mu \mathrm{M}$. The strong anti-dissociation effect of polyamines, especially of spermine, is considerable, as seen after $100 \mathrm{~s}$. Figure and data are kindly provided by Dr. Frank Peske. b: Structural formula of the used polyamines. Spermine is the polyamine with the longest chain length and the strongest basic character. The best identified stabilising condition for $70 \mathrm{~S}$ ribosomes seems to be related to these properties. The structural formulae were prepared with http://emolecules.com/.

\subsubsection{Biochemistry and Biophysical Strategies}

Sample integrity is a major prerequisite for successful structural analysis of any biological entity. For macromolecular complexes this is of special interest, as a complex, regarding low affinities or changed conditions during purification, can easily disassemble. In many cases small molecule additives can affect the compositional or conformational state of a complex. For example kirromycin, which binds to the interface of EF-Tu - GTP, was used to stall a codon recognition complex. Thereby a model was obtained exposing the interaction of the ribosome with the aminoacyl-tRNA and EF-Tu (Stark et al., 1997). Another antibiotic, fusidic acid, can be used to stabilise the EF-G bound posttranslocation complex (Stark et al., 2000). Further examples of stabilising additives are ATP, or in many cases non-hydrolysable analogues of ATP, like ATP $\gamma \mathrm{S}, \mathrm{AMPPCP}$ and others.

Beside specific additives, unspecific additives can stabilise a macromolecular complex. The polycationic polyamines spermine, spermidine and putrescine unspecifically interact with RNA and have been shown to reduce the conformational space of the ribosome, especially the $30 \mathrm{~S}$ rotation, to a similar extend as it is possible by GraFix (personal communication with Dr. Frank Peske and Dr. Niels Fischer). High throughput screens to measure stabilising conditions for protein complexes are very helpful to qualify the suitability of different buffer systems, $\mathrm{pH}$ values, and additives for certain protein complexes in a systematic way. Recently, a method called ProteoPlex (Haselbach, 2014) was introduced for macromolecular complexes, which is based on a stabilising assay for single proteins, namely Thermofluor (Ericsson et al., 2006). By heating a macromolecular complex in different buffer systems partial unfolding events can be monitored with dyes like SYPRO 
Orange. The aim is to find conditions, with late unfolding onset, while all subunits unfold in parallel, resulting in steep and regular unfolding curves. Thus, optimal conditions, possibly mimicking intracellular conditions, for a specific complex can be identified fast and cost-efficient. Nevertheless, not all dynamic event can be stabilised by the addition of a specific molecule and in some cases other approaches are necessary. Ndc80 contains very flexible coiled-coil domains, which were removed. Interestingly, the reduced dynamic readily allowed the growth of crystals, which was not possible in the full length complex (Ciferri et al., 2008). In many cases, similar genetic interventions may be necessary to modify a complex of interest in a way that it can be addressed by structural techniques. In this work, with cohesin, a very similar example is shown.

\subsubsection{Sample Preparation for Electron Microscopy (GraFix)}

Gradient fixation (GraFix) was introduced as a mild stabilisation protocol used for the structural determination of macromolecular complexes (Kastner et al., 2008). Many macromolecular complexes tend to aggregate, to disassemble partly or completely, making structural studies by any means impossible. In a gradient (composed of e.g. sucrose, galactose or glycerol), macromolecular complexes can be sorted during ultra-centrifugation according to their size. The compositional heterogeneity in the sample is reduced after fractionation. Simultaneous fixation within the complex stabilises the complex for structural analysis and is likely to reduce heterogeneity. Due to the gradient of the used fixation agent and the applied force during ultra-centrifugation, the tendency of cross-linking between different macromolecular complexes is reduced. Any containing aggregate migrates faster and accumulates at a deep fraction of the gradient.

In single particle EM, remaining gradient components will limit the image contrast. Thus, the buffer needs to be exchanged after the GraFix procedure. It was shown, that the overall appearance of a GraFix-treated sample is improved and that the tendency of disassembly and aggregation is reduced. Ultra-centrifugation parameters (speed, time) and gradient parameters can be adjusted for a specific particle size. As fixative agent, mainly glutaraldehyde (GA) is used, cross-linking $\epsilon$-amino groups in lysines, but to a lower extend also tyrosine, histidine and thiol residues. Further agents were tested, like P-maleimidophenyl isocyanate (PMPI). A cross-linking independent stabilisation was shown (personal communication with Dr. Ashwin Chari). PMPI revealed great performance, especially as a RNA-protein stabiliser, in an inverse gradient with increasing GA concentration and decreasing PMPI concentration from lighter to heavier fractions.

In this work, light (15\% glycerol) and heavy solutions ( $40 \%$ glycerol) were prepared (Figure 2.2). GA was added to the heavy solution $0.1 \%(\mathrm{v} / \mathrm{v})$, and PMPI was dissolved in DMSO and was added to the light solution at a final concentration of $1 \mathrm{mM}$. A gradient 
mixer was used to form a continuous gradient. The centrifugation was accomplished in a Sorvall Centrifuge Evolution R with a Sorvall TH660 rotor (speed: 45.000 rpm; time $960 \mathrm{~min}$ at $4^{\circ} \mathrm{C}$ for different complexes of 350-500 kDa). To limit sample heterogeneity, the GraFix temperature was reduced to $-10^{\circ} \mathrm{C}$ for specific samples, which will be noted accordingly in Chapter 3. For GraFix the buffer needs to be free of primary and secondary amins which was guaranteed by HEPES pH 8. The gradient solution was prepared as a $15 \%$ (v/v) (or $40 \%$ ) solution with $25 \mathrm{mM}$ HEPES pH 8, $150 \mathrm{mM} \mathrm{NaCl}$ and $2 \mathrm{mM}$ $\mathrm{MgCl}_{2}$. The ATP-bound state was trapped by addition of ATP $\gamma S$ (Jena Bioscience) at a concentration of $0.1 \mathrm{mg} / \mathrm{mL}(191 \mu \mathrm{M})$. The fixation was terminated by quenching with a final concentration of $10 \mathrm{mM}$ aspartate $\mathrm{pH} 7.9$ after gradient fractionation in $150 \mu \mathrm{l}$ fractions.

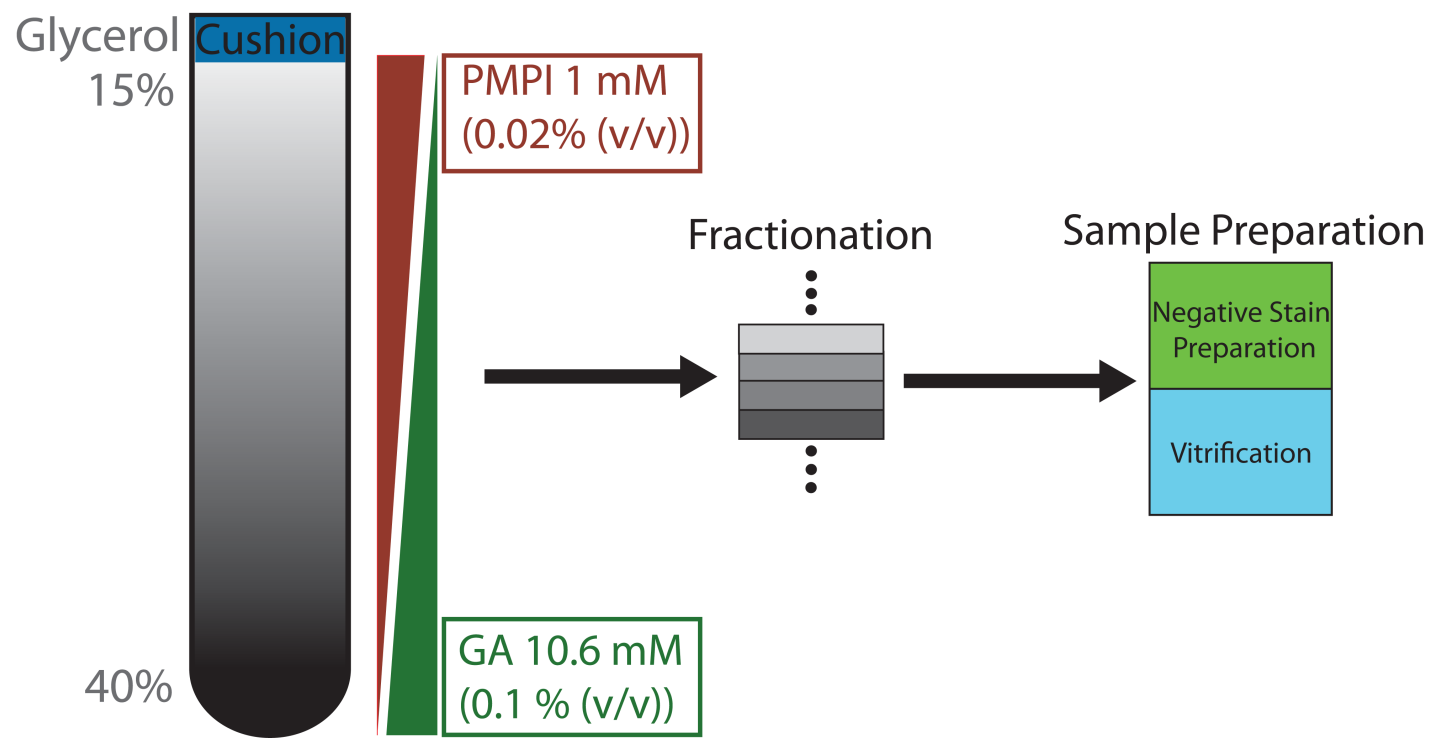

Figure 2.2: Sample Stabilisation with GraFix. On the right, a glycerol gradient from $15 \%$ to $40 \%$ glycerol is shown. Inside the glycerol gradient, an inverse PMPI and GA gradient is applied. A cushion is used to separate the loaded sample from the cross-linker. After ultracentrifugation, equally-sized fractions are prepared, to be screened in EM. Negative stain samples or vitrified samples can be prepared.

\subsection{Principles of Single Particle Electron Microscopy}

\subsubsection{Microscope}

Here, only a brief introduction into the theory of single particle electron microscopy will be given (according to Williams and Carter (2009)). The electromagnetic lens was developed by Hans Busch in the 1920s, making it possible to form an axially symmetric magnetic field, to focus electrons similarly to light in a glass lens. In 1931, Ernst Ruska and Max Knoll, manufactured the first electron microscope ${ }^{3}$. Already in 1933, the second

\footnotetext{
${ }^{3}$ Ernst Ruska was honoured in 1986, with the Nobel Price in Physics.
} 
generation electron microscope surpassed the resolution limit of light microscopy. Early on, electron microscopy contributed exceptionally to many fields of science, including material science and molecular biology. For example, studies on cellular organisation (mitochondria, viruses, ribosomes and many other biological entities) has been greatly improved by electron microscopy. In the following chapter, the basic physical principles of electron microscopy will be summarised (see Figure 2.3).

The spatial resolution of every magnifying system is limited according to the Rayleigh criterion, which is defined by the smallest distance to distinguish two dot-shaped points after passing through a circular aperture:

$$
\theta=1.22 \frac{\lambda}{D}
$$

where $\theta$ is the angular resolution (radian), $\lambda$ the wavelength and D the diameter of the lens' aperture. Accordingly, points are just resolved when the principal diffraction maximum of the first point matches the first minimum of the second point.

From this, the famous Abbe law can be derived:

$$
d_{\min }=\frac{0.61 \lambda}{n \sin (\alpha)} \approx \frac{\lambda}{2 n \sin (\alpha)}
$$

where $d_{\min }$ is the minimal distance to be resolved. The numerical aperture of the lens system is described by the refractive index (n) and half the included angle of the lens $\alpha$. The formula is simplified, assuming that the numerical apertures of objective and condenser are identical $(1.22 / 2=0.61)^{4}$.

According to 2.1 and 2.2 , it is obvious that a small wavelength contributes considerably to a high resolution. This is the main reason why electron microscopy is superior to light microscopy in terms of resolution ${ }^{5}$. In light microscopy, wavelengths of 400-800 $\mathrm{nm}$ are used, while the wavelength of electrons is much smaller (e.g. $\sim 0.0039 \mathrm{~nm}$ for $100 \mathrm{kV}$ ). According to wave-particle dualism, this wavelength can be determined for every particle by the de Broglie equation:

$$
\lambda=\frac{h}{m v},
$$

\footnotetext{
${ }^{4}$ The Rayleigh criterion and the Abbe law were developed independently, but are equivalent in their mathematical description. Different from Rayleigh, Abbe used not a circular aperture but a lattice. The value "1.22" is derived by applying the Bessel function $\left(J_{1}\right)$.

${ }^{5}$ This resolution criterion ignores the effect of lens defects in resolution (see Section 2.2.5).
} 
where $\mathrm{h}$ is the universal Planck constant of $\sim 6.626-34 \frac{\mathrm{m}^{2} \mathrm{~kg}}{\mathrm{~s}}$, $\mathrm{m}$ the mass and $v$ the velocity of the particle.

By applying a static electric field, an electron can be accelerated with a potential energy of the electron corresponding to the accumulated kinetic energy $(\mathrm{eV})^{6}$ :

$$
e V=\frac{m_{0} v^{2}}{2}
$$

$m_{0}$ describes the resting mass of the electron. Substituting the velocity $v$ by the momentum $\mathrm{p}$ in (2.4) and combining this with (2.3) results in:

$$
\lambda=\frac{h}{\left[2 m_{0} e V\right]^{\frac{1}{2}}}
$$

As close to the speed of light relativistic effects need to be taken into account, the following correction is included, yielding the approximation ${ }^{7}$ (Baker and Henderson, 2012):

$$
\lambda=\frac{h}{\left[2 m_{0} e V\left(1+\frac{e V}{2 m_{0} c^{2}}\right)\right]^{\frac{1}{2}}} \approx \frac{1.225}{\left[V\left(1+0.98 \cdot 10^{-6} V\right)\right]^{\frac{1}{2}}}[\mathrm{~nm}] .
$$

An electron microscope is composed of some universal components, which will be explained briefly. At the top of the microscope, there is a beam generating electron source. In principle, this source consists of an emitting material and an electron accelerating electric field. The condenser system, for many microscopes composed of two condenser lenses ${ }^{8}$, collects the electrons and ideally generates a parallel and coherent illumination of the specimen. In case of biological sample, the beam can only pass a thin specimen $(\sim 100 \mathrm{~nm})$ to allow high-resolution imaging. The image is formed by the objective lens and is further magnified by intermediate and projector lenses. Finally, the beam is detected by an electron detector. For direct optical visualisation, this is a phosphorescent screen made from particulate zinc sulphide. Historically, images have been converted chemically into a photographic film and digitised in a separate step. Today, images are detected and stored digitally using a charge-coupled device (CCD) (Faruqi and Subramaniam, 2000; Sander et al., 2005) or a direct electron detector (Faruqi and Henderson, 2007). There are several stigmators and deflectors positioned within the column to manipulate the beam. Gun tilt, gun shift, beam tilt, beam shift and image shift can be varied by the appropriate deflector within the column. Stigmators are used to make the beam circular in cross-section and correct the axial astigmatism. Inside the column, a very high vacuum is mandatory. Usually,

\footnotetext{
${ }^{6}$ In a static electric field, the electric charge is at rest, consequently no magnetic field exists.

${ }^{7}$ This can be approximated by the rule of thumb: $\lambda=(1.5 / V)^{(1 / 2)}[\mathrm{nm}]$

${ }^{8}$ In the case of Titan Krios, three condenser lenses are used.
} 
Figure 2.3: Composition of a TEM. A Philips Tecnai F20 TEM is depicted next to the schematic set-up of the microscope. All necessary elements are highlighted and some are related to the specific position within the Philips Tecnai F20 TEM. The sample holder inside of the compuStage is shown in purple. All beam deflectors and all stigmators are illustrated in blue and red, respectively. These blue and red elements are necessary for the microscope alignment. The detector, in this case a CCD camera, can be removed from the beam. The image was taken and adapted from ammrf, the Australian Microscopy \& Microanalysis Research Facility web presence. There, a virtual microscope is provided for training purposes (http: //www.ammrf .org.au/myscope/tem/ practice/virtualtem/basic.php).

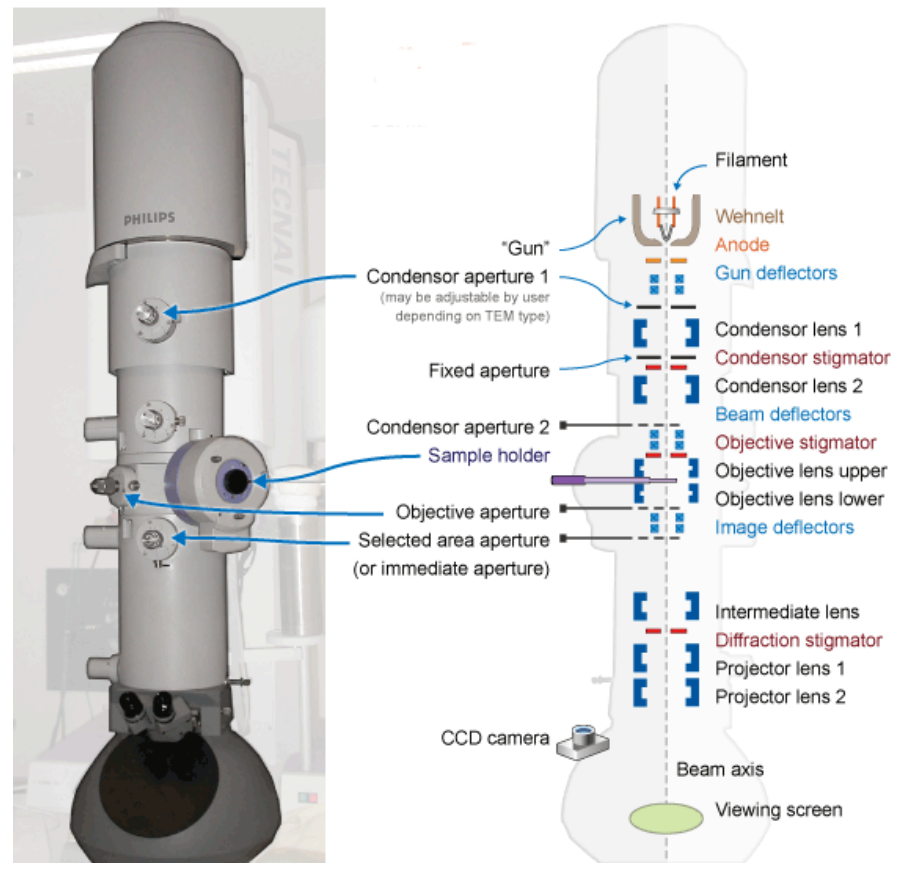

the vacuum forms a gradient (ranging from $10^{-5} \mathrm{~Pa}$ to $10^{-9} \mathrm{~Pa}$ at the susceptible field emission gun). While a very high vacuum is obligatory at the field emission gun, the weakest vacuum forms at the detector. To allow a satisfying mean-free path of the electrons, a vacuum below $10^{-1}$ is sufficient. By combining pre-vacuum pumps (e.g. mechanical rotary pumps), high vacuum (e.g. diffusion pumps) and ultra-high vacuum pumps (e.g. turbo pumps or ion getter pumps), adequate conditions are obtained. The sample (on a support grid) is loaded using a sample holder which is inserted into a compute-controlled stage (compuStage). In the stage, a precise $\mathrm{x}, \mathrm{y} \operatorname{shift}(\mu \mathrm{m})$ and tilt of the loaded sample is possible.

Electrons interact strongly with matter. In contrast to X-rays, they interact strongly with the electron cloud and the nuclei of atoms. These strong interactions provide a strong elastic signal, but additionally they induce radiation damage within the sample. Biological samples are exceptionally radiation-sensitive (Stark et al., 1996; Baker and Rubinstein, 2010). Accordingly, there are double-edged consequences of high electron doses, some very useful (e.g. signal strength, contrast, distinct readout signals) and some disadvantageous (e.g. beam-induced damage, heating and charging effects). Therefore, one needs to compromise between high contrast and protective conditions to preserve the high resolution information of the sample. Biological samples behave as so-called weak-phase objects and usually allow only very low signal-to-noise ratios (SNR). Typical SNR values at low-dose imaging conditions of cryo samples are around $10^{-1}$. Forward scattering of electrons is the most important signal used in TEM. Details on image formation in TEM will be explained in (2.2.7). 


\subsubsection{Electron Source}

In metals, electrons are bound weakly. If the neccessary energy is provided they can be emitted from the metal. To force electrons to leave the solid surface of metals, a certain work function $\Phi$, the minimum thermodynamic work, needs to be applied. Two possibilities, thermal emission and field emission, can be distinguished. Thermal emission results in a particular current density of the source $\mathrm{J}$ depending on the operating temperature $\mathrm{T}$ (Kelvin):

$$
J=A T^{2} e^{-\frac{\Phi}{k T}}
$$

where $\mathrm{k}$ is the Boltzmann constant $\left(8.610^{-5} \frac{\mathrm{eV}}{\mathrm{K}}\right)$ and $\mathrm{A}$, the Richard constant $\left(A / \mathrm{m}^{2} \mathrm{~K}^{2}\right)$, specific for the source material. Using a high temperature or a material with low work function $\Phi$ generates a sufficient current density. Therefore, only very heat-resistant materials are used, like tungsten (high melting temperature) or lanthanum hexaboride $\left(\mathrm{LAB}_{6}\right.$, with a low $\Phi)$. Depending on the used material and geometry, parameters like brightness, coherence, energy spread, working temperature and life time of the electron source can be very different. In this context, $\mathrm{LaB}_{6}$ crystals are superior compared to tungsten filaments. In thermionic emission, a so-called Wehnelt cylinder, a simple electrostatic lens, is used to accelerate the electrons by the applied electric potential. Newer microscopes use field emission guns (FEGs) because of the higher brightness, smaller energy spread and longer life time. It consists of a very sharp material tip where an extremely high electric field is applied $\left(>10^{9} \frac{\mathrm{V}}{\mathrm{m}}\right)$. Two anodes, one for extraction and the other for acceleration, are used within a FEG. This principle, based on quantum tunnelling, allows electrons to leave the material at reduced temperature and low work force. However, the vacuum requirements for a FEG are very high $\left(10^{-9} \mathrm{~Pa}\right)$.

A coherent beam results in in improved imaging conditions and reduced optical aberrations. It can be distinguished between temporal and spatial coherence. Temporal coherence defines a constant phase shift along the time axis comparing different waves, while spatial coherence refers to a constant phase shift orthogonal to the direction of propagation of the beam. To practically define the coherence, one can image a carbon film hole. Slightly out of focus, alternating dark and light rings, so-called Fresnel fringes, should appear near the edge of the carbon film. This effect is a typical wave interference pattern due to diffraction of the electron wave. More Fresnel fringes appear for a FEG in comparison to a thermionic source, as the beam is more coherent. 


\subsubsection{Electromagnetic Lenses}

Principally, electromagnetic lenses suffer from stronger optical aberration than glass lenses used in light microscopy. These aberrations are especially spherical and chromatic aberrations, so that small apertures let pass only the electrons closest to the optical axis, where the aberrations are small (Figure 2.4d). In general partial correction is performed by a procedure called coma-free alignment (Zemlin et al., 1978) and can be further improved by $C_{S^{-}}$correction (Haider et al., 1998).

Many laws from light optics apply for electron optics, like the Newton's lens equation:

$$
\frac{1}{f}=\frac{1}{d_{0}}+\frac{1}{d_{i}}
$$

where $\mathrm{f}$ defines the back focal length, $d_{0}$ the object plane and $d_{i}$ the image plane.

Electromagnetic lenses usually contain a cylindrically symmetrical soft iron pole piece. Within pole piece, is a cavity, namely the bore. Most lenses contain an upper and lower pole piece. Both contain copper coils, where a current is applied. This current defines the strength and direction of the magnetic field. By changing the current, the magnification can be changed.

Electrons are accelerated within the electromagnetic lens by the Lorentz force $(\mathrm{F})$ :

$$
F=-e(E+v \times B)
$$

where -e is the charge of the electron, E the strength of the electric field, B the strength of the magnetic field and $v$ the velocity of the electron. This rule obeys the right-hand rule, where the thumb points in the direction of the technical current flow, the forefinger points in the direction of magnetic field line and the middle finger in the direction of the Lorentz force. Accordingly, electrons spiral through the lens field on helical trajectories. Thus, the straight line illustration in electron ray diagrams are simplifications.

\subsubsection{Electron Detection}

In the beginning of TEM, images were detected by insertion of photographic film into the image plane. Due to the chemical reduction of silver, the image was chemically stored on the film. For computer processing, the films were scanned and stored digitally (Faruqi and Subramaniam, 2000; Faruqi and Henderson, 2007). This time consuming work can be circumvented by digital detectors (e.g. charge-coupled device (CCD) detectors). CCD detectors are based on the semiconducting properties of silicon (electron-hole pairs), 
and they allow charge separation to store a signal and charge transfer to read out the signal. The required energy of $3.55 \mathrm{eV}$ to produce an electron-hole pair is delivered by the incoming beam. However, in this case, immediate detection of the electron signal is adverse, as the dynamic range would be to low and beam-induced radiation damage would accumulate. Therefore, a protective scintillator layer of phosphor converts the electron signal into an adequate light signal for the CCD detector. It has been shown that CCDs behave superior to photographic film, especially at low-to-medium resolution, when binning is applied (Sander et al., 2005). Recently, direct detection devices (DDD) are replacing CCD detectors. There are two different techniques of DDDs, hybrid pixel detectors (HPD) and monolith active pixel sensors (MAPS). CMOS detectors, belonging to the MAPS, show the most promising features in terms of detective quantum efficiency (DQE) and SNR (according to Faruqi and Henderson, 2007). CCD detectors have an additional conversion step (and therefore an additional modulation transfer function), and, due to intrinsic light scattering, the SNR is low compared to direct detectors. Using CMOS chips, fast readout, counting mode and the recording of multiple frames is possible, which is especially interesting in the context of so-called beam-induced motion correction (Scheres, 2014; Glaeser and Hall, 2015).

\subsubsection{Microscopic Aberrations}

As mentioned already, electromagnetic lenses behave worse then light-optical lenses in terms of optical aberrations. In the following section, some relevant aberrations, namely spherical aberration $\left(C_{S}\right)$, chromatic aberration $\left(C_{C}\right)$, astigmatism $(A)$ and coma will be summarised $^{9}$ (Figure 2.4d) (according to Williams and Carter (2009)).

The $C_{S}$ relates to the difference in focus, depending on the distance from the optical axis. Waves further away from the optical axis have a shorter focal length, and therefore, any point object will be imaged as a disk of finite size. The disk size depends on the magnitude of $C_{S}$ and can be defined by:

$$
r_{s p h}=C_{S} \beta^{3}
$$

where $r_{s p h}$, is the radius of the spherically aberrant disk, $C_{S}$ is the lens-specific spherical aberration constant, and $\beta$ is the maximum angle of collection of the objective lens.

\footnotetext{
${ }^{9}$ To mathematically describe monochromatic wave front aberrations and their interdependency, Zernike polynomials are used. The total aberration can be expressed approximately by a linear combination of a limited number of basis elements. These basis elements are the principal aberrations of every optical system.
} 
Using a $C_{S^{-}}$-corrector designed for TEM by Rose and Haider, the third-order $C_{S}$ was considerably reduced. Principally, $C_{S^{-}}$correction adjust low-order aberrations on cost of high-order aberration. A correction limit is defined by the number of multipoles within the $C_{S}$-corrector. For a perfectly aligned microscope (having a beam parallel to the optical axis and reduced astigmatism), $C_{C}$ is the next most limiting aberration, defined by the energy spread of the incoming electrons (Haider et al., 1998). However, the energy spread can result from the electron source or the sample. The contribution of the electron source to $C_{S}$ is small (FEG: $0.3 \mathrm{eV}$ ), and corrections by monochromators are only reasonable if the contribution of the sample is low. The contribution of the sample depends on the thickness, and for thin samples, like in cryo EM, it can be neglected. Mathematically, $C_{C}$ can be described by:

$$
r_{c h r}=C_{C} \frac{\Delta E}{E_{0}} \beta
$$

where $r_{c h r}$ is the aberrant disk radius and $\Delta E$ the energy loss of the initial beam with the energy $E_{0} . C_{C}$ is the chromatic-aberration coefficient and $\beta$ defined as in (2.10).

A non-uniform magnetic field causes astigmatism due to microstructural inhomogeneities of every electromagnetic lens. As a result, two perpendicular electron planes will have different foci. These defects can be corrected by stigmators.

$$
r_{a s t}=\beta \Delta f
$$

where $r_{a s t}$ is the aberrant disk radius, $\Delta f$ the maximum different defocus induced by $\operatorname{astigmatism}(A)$.

The last distortion to be mentioned is coma. Any off-axial beam will induce a distortion, with a magnitude depending on the size of the incline. A point will be smeared out one-directionally, defining the name, derived from "comet".

Depending on these aberrations, the practical resolution in TEM is different from the $d_{\text {min }}$, defined according to the Rayleigh criterion, which assumes an aberration-free lens (Figure $2.4 \mathrm{~d}$ ). In a coma-free aligned microscope, the $C_{S}$ defines the resolution, and a compromised ideal $\beta$, which is very different for Rayleigh criterion and $C_{S}$, results in the following approximation:

$$
r_{\min } \approx 0.91\left(C_{S} \lambda^{3}\right)^{\frac{1}{4}}
$$




\subsubsection{Real Space and Fourier Space}

In many aspects of data analysis, transformation of data into another domain may be useful. One of these transformations which is used throughout this work is the Fourier transform. By a linear combination of an endless number of sinusoidal functions (sinus and cosinus), each function can be mathematically expressed. Images can be described similarly as two-dimensional function:

$$
f(t)=\int_{0}^{\infty}[a(v) \cos 2 \pi v t+b(v) \sin 2 \pi v t] d v
$$

Using the Euler formula, these sinusoidal functions can be presented in a complex form:

$$
f(t)=\int_{-\infty}^{\infty} F(v) e^{2 \pi i v t} d v \Leftrightarrow F(v)=\int_{-\infty}^{\infty} f(t) e^{2 \pi i v t} d t
$$

As an example, the transformation of data from frequency domain $v=1 / t$ into time domain $t$ is shown above. Similar images can be transformed between spatial frequency domain $v=1 / k$ and space domain $k$. The first mentioned formula above is the so-called inverse Fourier transform $(F(v) \rightarrow f(t))$. In reverse, $f(t)$ can be transformed into $F(v)$ using the so-called Fourier transform. Thus, these transforms just switch between real space $f(t)$ and Fourier space representation $F(v)$. In image processing, it is useful to plot a noisy 2D image in Fourier space. High-spatial frequencies (short distance features in real space like random noise) will be located at the outer circle of the image, while low-spatial frequencies (long distance features in real space) will be located closer to the center. High-spatial frequencies, which may correspond to dispensable data, can be removed easily by applying a mask in Fourier space.

Any sinusoidal wave can be described sufficiently by its amplitude $(A)$ and by its phase $(\phi)$. The complex formulation above is especially useful to represent $A$ and $\phi$ by the combination of a real and an imaginary part:

$$
\begin{gathered}
|A|=\sqrt{\text { real }^{2}+\text { imaginary }^{2}} \\
\tan \phi=\frac{\text { imaginary }}{\text { real }}
\end{gathered}
$$




\subsubsection{Image Formation and Contrast Transfer Function (CTF)}

In Figure 2.4a, all signals generated by electron radiation of matter are illustrated. These signals can be used for different purposes. Scanning electron microscopy (SEM) uses the backscattered electrons (BSE) and secondary electrons (SE) as readout signal. Elementary analysis is possible by analysing the emitted characteristic X-rays. However, this section focuses on elastically and inelastically scattered electrons (Figure 2.4b), which are the interesting signals for contrast formation in TEM (Figure 2.4a). Interestingly, even if radiation damage is a limiting event in cryo EM, it is several orders of magnitude lower than for a similar X-ray scattering experiment (Henderson, 1995).

a

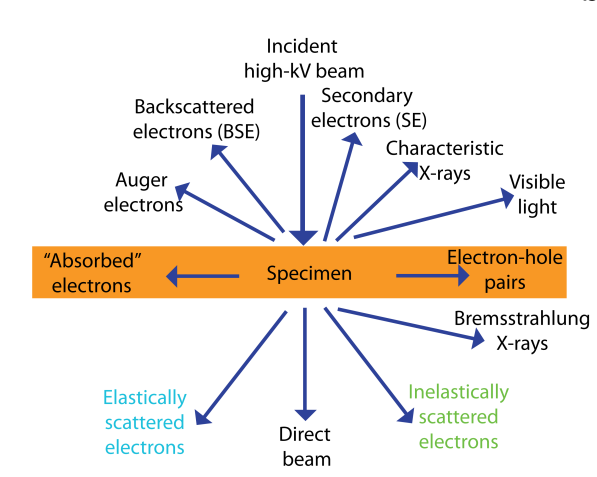

C

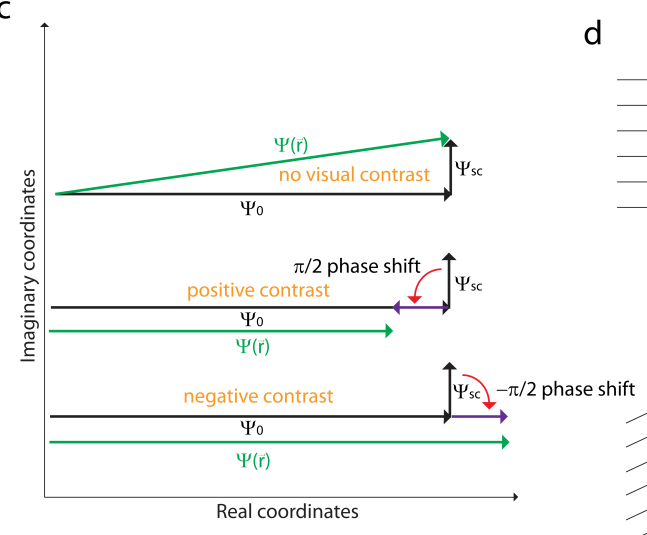

b

d
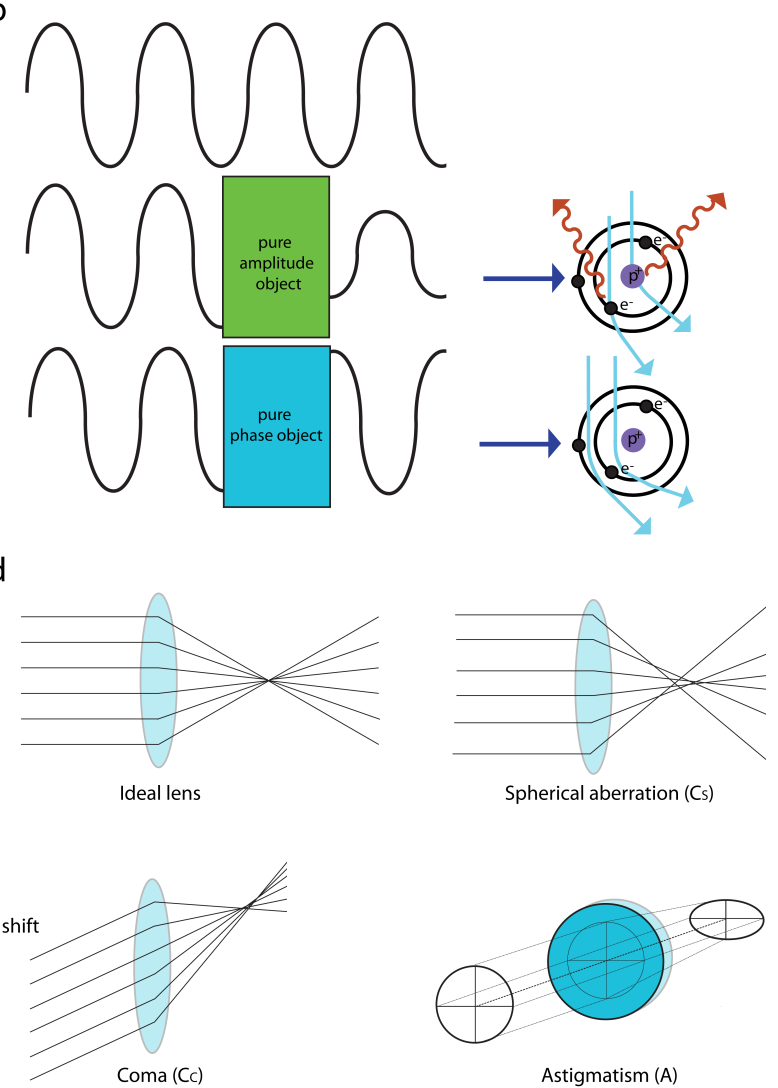

Figure 2.4: Concepts of Image Formation. a: Very different signals are generated by a high-energy electron beam, which can be detected with varying detectors within the microscope. In single particle EM, elastically scattered and inelastically scattered electrons are relevant for image formation. The image was adapted from (Williams and Carter, 2009, Chapter 1.2.C, Figure 1.3). b: Depicted is the interaction of an electron wave with matter in two cases, distinguishing between a theoretical pure amplitude object (green) and a pure phase object (blue). Energy transfer between the electron wave and a local nucleon or electron reduces the amplitude of the wave, which can be recognised in amplitude contrast. The electron is inelastically scattered. In contrast, phase contrast refers to elastic scattering events. In this case, no energy is transferred and a phase shift of $\pi / 2$ is obtained (imaginary component). However, to visualise the phase object, an additional phase shift of $\pi / 2$ needs to be applied (real component). c: In electron microscopy, the additional phase shift is not obtained by a ring-like $\lambda / 4$ Zernike phase plate like in light microscopy but by image defocusing. Thereby, a positive or a negative phase shift can be obtained, which is visible in real space (Kohl et al., 2007). d: Electromagnetic lenses are limited by strong aberrations. Here, the effect of spherical aberration $\left(\mathrm{C}_{S}\right)$, coma $\left(\mathrm{C}_{C}\right)$ and astigmatism $(\mathrm{A})$ is illustrated. 
The minimum requirement to distinguish two points of equal intensity in a 2D image is their separation by at least one pixel of different intensity $(I)$. Low contrast can limit the discriminability of two points. Contrast $(\mathrm{C})$ is defined by the intensity difference between the brightest and the darkest point, normalised by the overall intensity, according to Michelson contrast as:

$$
C=\frac{I_{\max }-I_{\min }}{I_{\max }+I_{\min }}
$$

Inelastic scattering arises upon energy loss of the incoming electron due to the interaction with the coulomb potential $(\varphi)$ of nuclei within the sample. This energy loss usually relates to a huge scattering angle. Thus, the electrons are removed by hitting the objective aperture. Additionally, electrons may be completely absorbed by multiple scattering events. These inelastic scattering events contribute to the so-called amplitude contrast. It was shown that most scattering events are actually inelastic for light atoms as in biological samples (Henderson, 1995). Negatively stained particles result in an even higher contribution of amplitude contrast. Therefore, amplitude contrast will always contribute to the image, especially in the low-spatial frequency domain. This is not the favoured form of image contrast, as it provides an undesirable background term in the image. Additionally, the loss of energy of the electron relates to radiation damage (e.g. free radical formation and ionisation) of the sample. Thus, the decay of the sample originates from those inelastic scattering events. Consequently, one needs to work at low dose conditions ( $20 e^{-} / \AA^{2}$ ), limiting the signal-to-noise ratio (SNR) remarkably.

The second form of contrast derives from elastic scattering events of the electrons within the sample. Due to elastic scattering, no energy is transferred (Figure 2.4b). A pure phase object shifts the incoming electron wave proportional to the integral of the coulomb potential. Accordingly, the phase shift depends on the atomic number $(Z)$. However, the phase shift is much smaller than the electron wavelength and therefore not visible. In light microscopy, a ring-like $\lambda / 4$ Zernike phase plate is used to achieve an additional phase shift of $\pi / 2$ on the non-scattered light beam, so that the interference of the scattered and the non-scattered light beam generates maximum contrast. So far, the developed TEM phase plates do not satisfy the standard requirements in single particle EM routine (Nagayama and Danev, 2009; Nagayama, 2011), as an insertion of any material into the beam can generate severe beam aberrations due to accumulated contaminations. Differently, by defocusing the spherical aberration, $C_{S}$ can be used to generate an additional phase shift (Figure 2.4c). The contrast transfer of varying spatial frequencies differs for a certain defocus $\Delta$. An optimal defocus is defined by the so-called Scherzer focus $-1.2\left(C_{S} \lambda\right)^{1 / 2}$, with the maximum positive phase contrast prior to the first zero crossing of the contrast transfer function. For thin samples, the weak-phase approximation holds true where 
contrast formation is understood in terms of phase contrast by thin specimen of low atomic number $(Z)$. The image signal is proportional to the amplitude of the scattering and linearly related to the object property. The term "weak-phase-object" for a biological specimen derives from the fact that the generated phase shift of a thin specimen of low $Z$ is very small. The following derivation is depicted according to Orlova and Saibil (2011):

The phase shift $\varphi(\vec{r})$ is defined as:

$$
\varphi(\vec{r})=\int_{-t / 2}^{t / 2} C(\vec{r}, z) d z
$$

where $\vec{r}$ is a $2 \mathrm{D}$ vector $\vec{r}=\left(\begin{array}{l}x \\ y\end{array}\right)$ and $C(\vec{r}, z)$ the $3 \mathrm{D}$ coulomb potential distribution within the object of a thickness $t$. The phase shift $\varphi$ is proportional to the integral of $C(\vec{r}, z)$.

Accordingly, the incoming wave $\Psi_{0}$ is modified as follows :

$$
\Psi(\vec{r})=\Psi_{0} e^{i \delta \varphi(\vec{r})}
$$

$\delta$ is a constant ${ }^{10}$

According to Euler's formula and the weak-phase approximation $(\varphi(\vec{r})<<1)$, the formula can be simplified as follows, where the higher-order terms can be omitted:

$$
\Psi(\vec{r}) \approx \Psi_{0}(1+i \delta \varphi(\vec{r}))
$$

In this form, a decomposition into a non-scattered term $\Psi_{0}$ and a scattered term $i \delta \varphi(\vec{r}) \Psi_{0}$ becomes obvious. However, the given phase contrast of thin and unstained biological sample is weak, explaining the term "weak-phase object". Mathematically, this weak phase contrast can be expressed as:

$$
I(\vec{r})=\Psi(\vec{r}) \cdot \overline{\Psi(\vec{r})} \approx 1+(\delta \varphi(\vec{r}))^{2}
$$

\footnotetext{
${ }^{10} \delta$ is proportional to the mass of the electron $m_{e}$ and the electron wavelength $\lambda$. The denominator term is proportional to the second power of Planck's constant $\hbar$ :

$$
\delta=\frac{m_{e} \lambda}{2 \pi \hbar^{2}},
$$


As $(\delta \varphi(\vec{r}))^{2}<<1$, no visible contrast can be observed. For that reason, an additional phase shift of the scattered beam by $90^{\circ}(-\pi / 2)$ needs to be applied:

$$
\Psi(\vec{r}) \approx \Psi_{0}(1-i \delta \varphi(\vec{r}))
$$

Furthermore, the intensity distribution $I(\vec{r})$ results in:

$$
I(\vec{r})=\Psi(\vec{r}) \cdot \overline{\Psi(\vec{r})} \approx 1-2 \delta \varphi(\vec{r})
$$

The term $\overline{\Psi(\vec{r})}$ is the complex conjugate of $\Psi(\vec{r})$. The magnitude of the term $1-2 \delta \varphi(\vec{r})$ is much greater than the term $1+(\delta \varphi(\vec{r}))^{2}$, where no additional phase shift is applied. Accordingly, without the phase shift, almost no contrast would be visible.

In a perfect objective lens, the electron wave would form the physical equivalent of the Fourier transformation $F\left(\Psi_{o b s}(\vec{r})\right)(2.20)$ at the back focal plane. However, an additional term containing a wave aberration function of an imperfect lens needs to be included. In reciprocal space, this is expressed by the phase contrast transfer function $(\mathrm{CTF})$ :

$$
F\left(\Psi_{o b s}(\vec{r})\right)=F(\Psi(\vec{r})) \cdot F(P S F((\vec{r}))=F(\Psi(\vec{r})) \cdot C T F(\vec{R}) \cdot E(\vec{R})
$$

where $F\left(\Psi_{o b s}(\vec{r})\right)$ is the Fourier transform of the observable image and $F(P S F((\vec{r}))$ is the Fourier transform of the point-spread function $(P S F((\vec{r}))$, which consists of the $C T F$ and an envelope function $E(\vec{R})$ in real space. The envelope function results from the specimen decay under the beam and several further distortions. $\vec{R}$ is the vector of the spatial frequencies (Fourier space coordinate). Expressed differently, the exiting wave is convoluted (and thereby degraded) by a PSF:

$$
\Psi_{o b s}(\vec{r})=\Psi(\vec{r}) \star P S F(\vec{r})
$$

The phase shift $\varphi(\vec{R})$ of the spatial frequency $\vec{R}$ is defined as:

$$
C T F(\vec{R})=e^{-i \varphi(\vec{R})}, \quad \text { with } \quad \varphi(\vec{R})=\frac{\pi}{2}\left(C_{s} \lambda^{3} \vec{R}^{4}-2 \Delta \lambda \vec{R}^{2}\right)
$$

The latter expression is the so-called Scherzer formula. In this context, it is interesting to define generalised defocus $\Delta z_{\text {gen }}$ and spatial frequencies $k_{g e n}$, which can be applied for all TEMs independent of instrument-specific lens aberration: 
Figure 2.5: Effect of Defocusing on the Phase Contrast Transfer Function (PCTF). The PCTF is shown as a function of intensities and spatial frequencies in dependency of varying defocus $(0.5 \mu \mathrm{m}, 1 \mu \mathrm{m}, 2 \mu \mathrm{m}, 4 \mu \mathrm{m}$ and 10 $\mu \mathrm{m})$. All other parameters are constant. Increasing defocusing results in more frequent zero crossings and intensity inversion within the PCTF. Accordingly, a $\mathrm{CTF}$ correction is necessary to restore the inherent information (compare Figure 1.32 and Chapter 2.5.1.2). The envelope function $E(\vec{R})$ results from specimen decay and further distortions. The presented graphs were simulated with Imagic software package (van Heel et al., 1996).

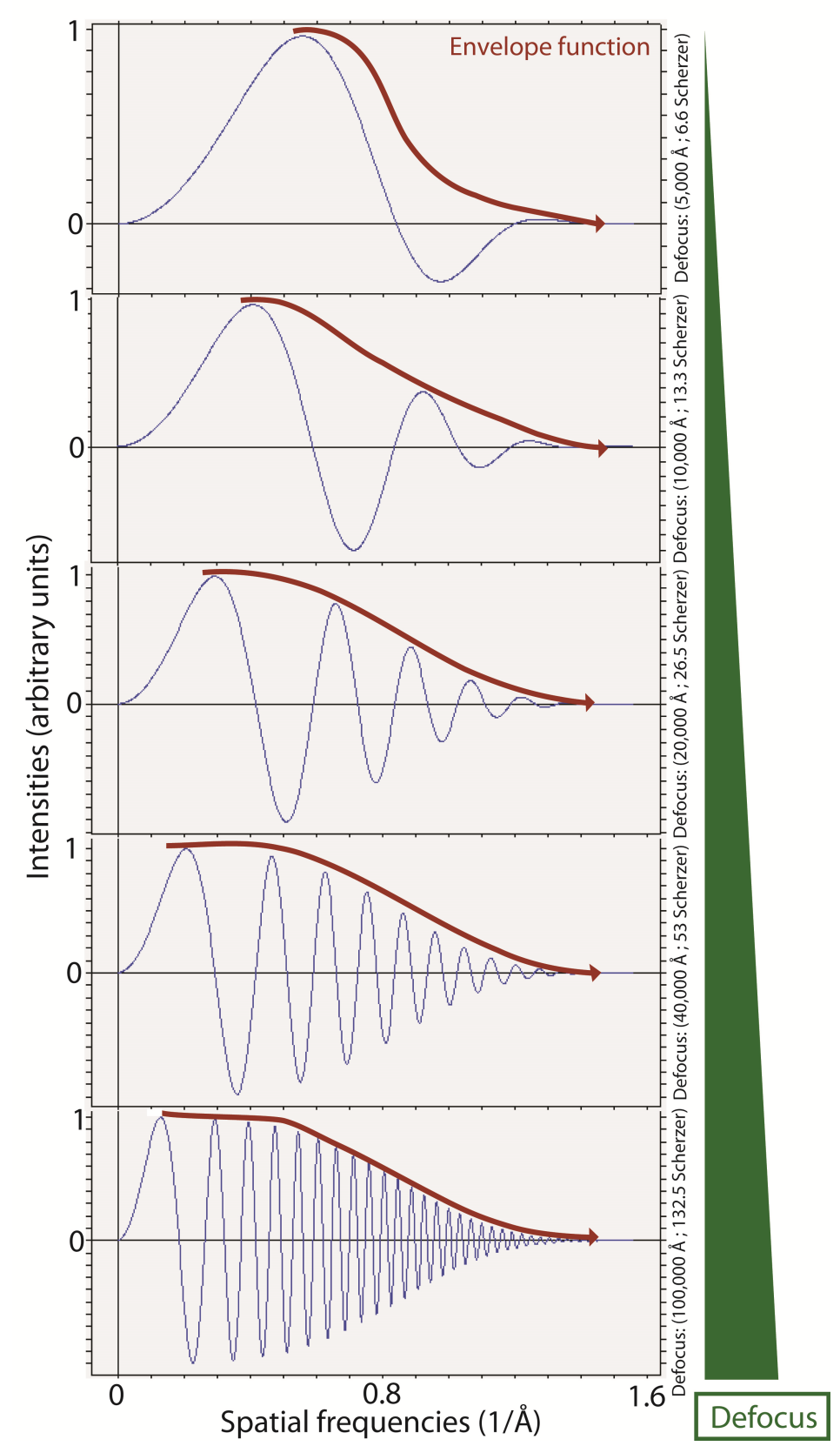

$\Delta z_{\text {gen }}=\frac{\Delta z}{\left(C_{S} \lambda\right)^{1 / 2}} \quad$ and $\quad k_{\text {gen }}=\left(C_{S} \lambda^{3}\right)^{1 / 4} k$

\subsection{Sample Preparation for Single Particle EM}

\subsubsection{Negative Stain Sample Preparation}

Negative stain sample preparation is an extensively used preparation technique (Brenner and Horne, 1959), where the sample is embedded in a heavy metal stain solution, usually 
uranyl acetate or uranyl formate. As the contrast is derived by the stain, the particles appear light and the background dark. Additionally, no real projection image is recorded but the shape of the complex of interest, defined by the surrounding stain. Due to the strong contribution of amplitude contrast, a strong contrast signal is possible. Additionally, induced beam damage is a minor problem compared to vitrified sample. Homogeneous staining is of great importance for the possible image quality, especially when images are tilted. All particles should be covered with stain, while the stain should be neither too thick, resulting in lower contrast, nor too thin, leading to positive staining. It is important to consider that the stain can change the appearance of the particle (e.g. sample flattening). Negative stain sample preparation results in a resolution limit. The graining size of the stain defines the maximal resolution, which is usually in the 10-25 A range. By cryo-negative staining, where a low concentration of aqueous staining solution is vitrified, the possible resolution may be increased. The sample is not dried and the contribution of the sample to the contrast is stronger than in negative stain (Adrian et al., 1998; Golas et al., 2003).

A $2 \%(\mathrm{w} / \mathrm{v})$ stain solution is freshly prepared in double-distilled water as the stain gradually precipitates due to UV light. The solution is mixed and the precipitate is pelleted by 15 min of centrifugation at $13000 \mathrm{rpm}$. A carbon film is floated on top of a protein solution for adsorption of protein complexes onto the film. The adsorption time depends on the used complex concentration. The absorption time is usually in the range of about $1 \mathrm{~min}$ with sample concentrations of about $100 \frac{\mathrm{nmol}}{\mathrm{l}}$. A holey carbon film-coated grid is used to adsorb the carbon film with the particles and is transferred for $1 \mathrm{~min}$ into the uranyl formate solution. The excess of liquid is blotted away with wet filter paper, and the grid is dried at room temperature.

\subsubsection{Cryo Sample Preparation}

The preparation of macromolecules in vitrified ice was a breakthrough in structural EM (Dubochet and McDowall, 1981). Different from negatively stained sample, here the image contrast originates from the sample itself. Vitrification is the transition from liquid into an amorphous glass-like condition. Vitrified ice can be formed by ultra-fast cooling rates, preventing the formation of ice crystals. By plunging very thin water films into liquid ethane, cooling rates of $\frac{100,000 K}{s}$ are possible, transforming a $0.1 \mu \mathrm{m}$ thick water layer in a few microseconds into the vitrified state (Al-Amoudi et al., 2004). Today, for plunging, automated systems with controlled humidity and temperature, like the Vitrobot, can be used. The humidity is always adjusted to $100 \%$. Additives with similar density as the particles of interest will decrease the contrast in vitrified samples. Accordingly, any gradient additives (e.g. glycerol, sucrose) from GraFix need to be removed (e.g. by Zebra 
spin desalting columns). Similarly as in negative stain preparation, the macromolecular complex is first attached to thin carbon film. Subsequently, the foil is adsorbed onto a grid (e.g. QUANTIFOIL) ${ }^{11}$. Plunge freezing can be performed manually or automatically. In this work the prepared grids were loaded into the Vitrobot apparatus and $4 \mu \mathrm{l}$ of water are applied onto the sample site. Filter paper within the Vitrobot apparatus is used to aspirate the excess of water, while blot force and blot time can be freely adjusted to optimise the thickness of ice. After a defined waiting time, plunge freezing in liquid ethane is performed. The grids can be stored in liquid nitrogen. Differently, for ribosome sample, a pre-floated grid $^{12}$ is prepared prior, and, directly before freezing, $4 \mu \mathrm{l}$ of the sample is applied within the Vitrobot apparatus with an appropriate incubation time (30 s). This standardised work flow is possible due to the known concentration of the used ribosome sample $(0.034$ $\mu \mathrm{M})$. By glow discharging, the affinity of the particles to the pre-floated grids needs to be increased. In all cases of ribosome vitrification, the temperature is adjusted to $20^{\circ} \mathrm{C}$ within the Vitroblot.

\subsection{Image Acquisition}

Image recording was performed with a Philips CM200 FEG microscope (4Kx4K CCD camera, Tietz Video Systems) or with a Titan Krios microscope equipped with a $C_{S}$ corrector and two different detectors systems (CCD: Eagle and CMOS: Falcon2). An automated image acquisition system used for Titan Krios allows to predefine useful image positions, while imaging can be performed overnight without user interference. The $C_{S}$ corrector is used for alignment to minimise image distortion related to astigmatism, coma or spherical aberration (Haider et al., 1998). The usage of the Titan Krios allows to generate datasets in the range of 300.000 particles overnight. At the CM200, a semi-automated software (TVIPS EM-MENU) is used, which allows to generate a predefined number of micrographs per selected position. In this work, a 3x3 or 4x4 array was used, generating 9 or 16 micrographs per position without overlap. However, the usage is time-consuming. Usually, datasets in the range of 10.000-100.000 images were recorded. At the CM200, tilt-pairs were generated with a $20 \%$ overlap to combine the images into one joined file. The applied tilt-angle for tilt-pairs was $20^{\circ}$ or $45^{\circ}$.

Image acquisition parameters are defined in the table below. Ribosome cryo datasets were recorded at Titan Krios with the Falcon2 detector (CMOS) or Eagle detector (CCD) (Table 2.5). Here, the defocus was varied between 1 and $2 \mu \mathrm{m}$, and the dose was adjusted

\footnotetext{
${ }^{11}$ QUANTIFOIL grids are commercially available grids with pre-assembled thick perforated carbon film and a continuous hole pattern. In this work only QUANTIFOIL R 3.5/1 are used.

${ }^{12}$ In this case, the carbon film is attached to the grid before the sample is loaded. The film adsorption onto the grid can be achieved on the surface of double distilled water.
} 
to 25 electrons per $\AA$. Datasets of negatively stained sample (cohesin) were recorded at CM200 (88k) or Titan (37k, $80 \mathrm{KV})$. At CM200, the defocus is adjusted manually between 1 and $3 \mu \mathrm{m}$. Cohesin cryo datasets were recorded equivalently to ribosome datasets.

Table 2.4: Image acquisition at Philips CM200

\begin{tabular}{|l|l|}
\hline Description & Properties \\
\hline Magnification & $88 \mathrm{k}$ \\
\hline Pixelsize & $2.45 \AA / \mathrm{p}$ \\
\hline Binning & 2 -fold \\
\hline Pixel & $2048 \mathrm{x} 2048$ \\
\hline Detector & $4 \mathrm{Kx} 4 \mathrm{~K}$ CCD (Tietz Video Systems) \\
\hline High tension & $160 \mathrm{kV}$ \\
\hline Sample condition & Negative stain \\
\hline
\end{tabular}

Table 2.5: Image acquisition at FEI Titan Krios

\begin{tabular}{|l|l|l|l|}
\hline Description & \multicolumn{3}{|c|}{ Properties } \\
\hline Magnification & $37 \mathrm{k}$ & $37 \mathrm{k}$ & $47 \mathrm{k}$ \\
\hline Pixelsize & $1.71 \AA / \mathrm{p}$ & $2.02 \AA / \mathrm{p}$ & $1.57 \AA / \mathrm{p}$ \\
\hline Binning & 2 -fold & 1 -fold & 1 -fold \\
\hline Pixel & $2048 \times 2048$ & $4096 \mathrm{x} 4096$ & $4096 \times 4096$ \\
\hline Detector & $4 \mathrm{Kx} 4 \mathrm{~K}$ CCD (Eagle) & $4 \mathrm{Kx} 4 \mathrm{~K}$ CMOS (Falcon2) & $4 \mathrm{Kx} 4 \mathrm{~K}$ CMOS (Falcon2) \\
\hline High tension & $80 \mathrm{kV}$ & $300 \mathrm{kV}$ & $300 \mathrm{kV}$ \\
\hline Sample condition & Negative stain & Cryo & Cryo \\
\hline
\end{tabular}

\subsection{Image Processing}

\subsubsection{D Level}

In the following section, the 2D image processing will be introduced. Thereafter, the 3D model building from 2D images will be elucidated. Finally, different methods for structural validation will be explained.

2D images can be described by a matrix $M$ where position $\left(x_{n}, y_{n}\right)$ and grey-value $\left(g_{n}\right)$ of each pixel are stored with an appropriate counting index:

$$
M=\left(\begin{array}{cccc}
x_{1} & y_{1} & 0 & g_{1} \\
\vdots & \vdots & \vdots & \\
x_{n} & y_{n} & 0 & g_{n}
\end{array}\right)
$$

For most purposes of image processing, like alignment and classification, this is the most convenient way, while for simple image statistics a vectorial or sequential illustration is sufficient. 


\subsubsection{Particle Selection}

The recorded micrographs should contain an appropriate density of particles which are extracted to generate a stack of single particle images. This can, depending on the optical contrast appearance, be tedious, especially for images of vitrified particles if they are small and heterogeneous. However, it is a very critical step, as it should be avoided to be biased by non-particle images (e.g. pure noise, degraded or aggregated particle, ice or ethane artefacts and negative stain artefacts) in the following processing steps (Henderson, 2013). Different strategies can be applied depending on the appearance, quality and size of the dataset. Particle selection can be performed manually, which is very time-consuming, or by semi-automated or fully automated procedures. Various approaches, like template matching (Chen and Grigorieff, 2007), statistic approaches (Voss et al., 2009), machine learning algorithms (Langlois et al., 2011) or combinations (Zhao et al., 2013) are used, which can speed up the extraction (Zhu et al., 2004). However, visual evaluation of raw images or calculated class averages is useful to evaluate the extraction quality. Post-extraction data cleaning steps on class average level can remove unwanted images. Usually, the extracted area covers two to three times the diameter of the particle, as the background noise is useful for image normalisation and CTF correction. In the used in-house software cowPicker (Busche, 2013), particles are interpreted as mass, attracting the selection points iteratively towards the particle. Initially, the points are positioned on an equidistant grid. The automated selection can be optimised for a specific particle by changing parameters like the threshold of the local variance and the mean or the threshold of the local density ratio. Manually, the selection of each micrograph can be adjusted according to the user's notion.

\subsubsection{Image Restoration}

As shown in Section 2.2.7, the CTF oscillates between positive and negative values. Thus, each time the algebraic sign changes, the zero position is crossed, resulting in those spatial frequencies being omitted. Moreover, due to the envelope function $E(\vec{R})$, the signal amplitude dampens for high-spatial frequencies. The rate of zero crossings increases with rising spatial frequencies, depending on the applied defocus. This can be observed in the image power spectrum $p s(\vec{r})$, by squaring the magnitude of the frequency component $\left|F\left(\Psi_{o b s}(\vec{r})\right)\right|^{2}$. Here, the oscillation manifests in the alternating black and white rings, the so-called Thon rings. The missing information at the zero crossings, as well as the signal dampening for high-spatial frequencies, limit the usability of these data. Therefore, the image can be restored by numerically defining the CTF. Thus, for each image the spherical aberration $\left(C_{S}\right)$, the defocus $(\Delta)$, the B-factor and the magnitude and direction of astigmatism (A) can be calculated. In a process called phase flipping, all 
spatial frequencies obtain a positive sign by multiplication of $F\left(\Psi_{o b s}(\vec{r})\right)$ with the algebraic sign of the calculated CTF. The zeros are still there, but the exact position (specific spatial frequencies) varies with different defoci. Thus, restoration of the missing information is possible by averaging several images with distinct defoci. The enevelope function $E(\vec{R})$ can be corrected by multiplying the image with an inverse B-factor decay curve $e^{-\frac{B}{4 d^{2}}}$, in a process called "Sharpening". Here, $d$ is the real space coordinate. Principally, amplitudes can be defined experimentally by X-ray scattering as well.

The particle image stack needs to be further processed on single image level. Depending on the contrast features and the expected resolution, downsampling ${ }^{13}$ of the image is useful for two reasons. By downsampling, the particle contrast can be increased relative to random noise within the image. Additionally, the image processing (e.g image alignment) can be sped up due to the decreased data size. During refinement, the downsampling level should be reduced, as otherwise the maximal resolution remains limited. According to the Nyquist-Shannon sampling theorem, the maximal possible resolution is twice the pixel size. In practice, a factor between two and three is more realistic ${ }^{14}$.

Not all spatial frequencies do correlate to features of the particle and consequently can limit the performance of subsequent processing steps. Accordingly, they should be removed from the image. As shown in Section 2.2.6, the representation of an image in reciprocal space is helpful to remove specific undesired spatial frequencies of the image by applying a mask (Figure 2.6). These adverse spatial frequencies relate largely to certain types of noise. Image gradients (e.g. resulting from ice thickness or stain thickness) are typical in the low-spatial-frequency domain, while random noise is specific for the high-spatialfrequency domain. Thus, a bandpass filter can be applied in order to reduce these types of noise. However, high-spatial frequency information may correlate to the particle's details and cannot be distinguished from random noise. In consequence, during on-going image processing and refinement, the filter parameters can be adapted in the original file in order to enhance the high-spatial-frequency domain. In initial alignments, low-spatial frequencies are more important than in late alignment iterations, so that the high-spatial frequency cut-off can be relatively low. The following formulae are helpful to adjust the filter cut-off at low-spatial (LF) and high-spatial (HF) frequency domain according to the particle size and the expected resolution:

$$
\text { LF cut-off }=\frac{2 \cdot \text { pixel size }}{\text { particle size }} \text { and } \quad \text { HF cut-off }=\frac{2 \cdot \text { pixel size }}{\text { expected resolution }}
$$

\footnotetext{
${ }^{13}$ Referred to as "coarsening" in practice.

${ }^{14} \mathrm{~A}$ meaningful pixel size should already be decided in earlier steps, as microscopic magnification and image recording parameters (e.g. binning) cannot be reversed.
} 
In order to be free of "overshoot" artefacts in Fourier space, one should never apply a hard cut-off, but always a transient cut-off. Therefore, different filter algorithm used, like the Gaussian filter, the Fermi filter, the adaptive Wiener filter or the Butterworth filter (Frank, 2006, Appendix 2).

The average grey-values of two images can vary depending on stain thickness, carbon film thickness and changing image recording conditions. Thus, images need to be normalised relative to each other before they are compared or integrated via image cross-correlation or image summation, respectively. This is achieved by defining a new mean and a general standard deviation for the whole image set. Here, the mean is set to zero and the standard deviation set to ten.

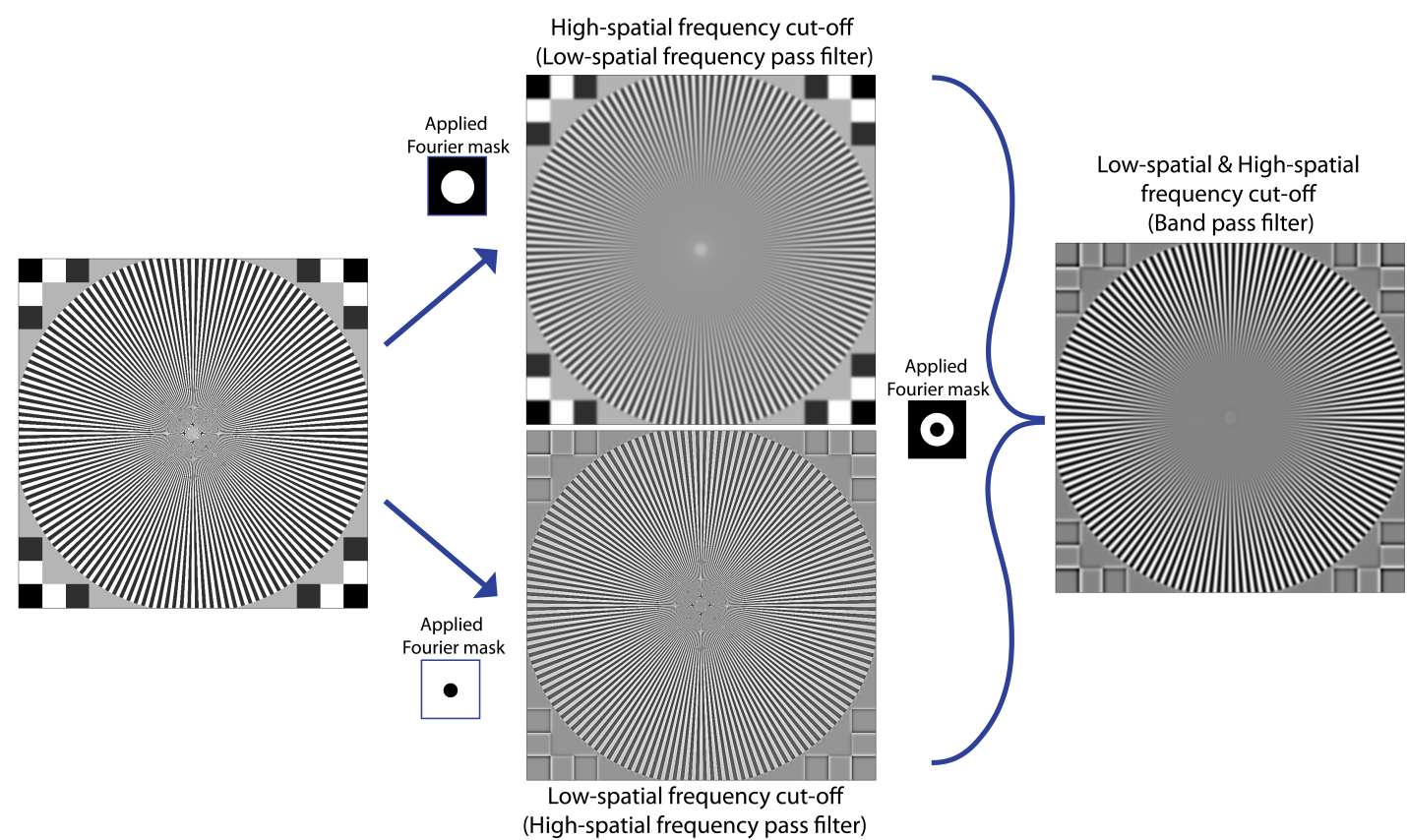

Figure 2.6: Application of Fourier Filters. The effect of Fourier filtering is shown exemplary for a Siemens star. In the Siemens star, the spatial frequencies are constant within a certain radius and are gradually changing with varying radius. Accordingly, the effect of applied Fourier filters can be visualised. In the upper case, a low pass filter is applied, removing all high-spatial frequencies. In the lower case, low-spatial frequencies are removed with a high pass filter. The combination of both filters results in a band pass filter. Filtering can be performed in Fourier space by the application of specific masks, as illustrated. By this procedure, unspecific low-spatial and high-spatial noise can be partly removed.

\subsubsection{Image Averaging}

Single particle EM images suffer from low SNR $\left(10^{-1}\right)$. Summing low SNR projection images of particles in the same perspective increases the SNR by a factor of $\sqrt{N}$, with $N$ being the number of averaged images. Accordingly, the information accumulates, while noise is averaged out. Thus, even high-spatial frequency information can be restored.

The signal-to-noise ratio (SNR) of an image is defined as: 


$$
S N R=\frac{P_{\text {signal }}}{P_{\text {noise }}}
$$

where $\mathrm{P}$ is the integral of the power spectrum after normalisation.

Before averaging equal projections, they need to be identified, despite occurring shifts and rotations. Three perpendicular translational $(X, Y, Z)$ and three perpendicular rotational $(\alpha, \beta, \gamma)$ degrees of freedom, so-called Euler angles, are sufficient to describe every possible position and orientation of a $3 \mathrm{D}$ object in $3 \mathrm{D}$ space ${ }^{15}$. Many different projectional views of a given 3D object are to be expected from randomly oriented sample, although some macromolecular complexes have preferred orientations due to electrostatic interactions of the macromolecular complexes with the carbon film. Before images can be integrated, similar projections (similar $\beta, \gamma$ ) need to be grouped and aligned according to their translational $(X, Y)$ and in-plane rotational $(\alpha)$ position.

\subsubsection{Alignment of Images}

During image alignment, the best way to correctly superimpose two images is defined by sampling the translational $(x, y)$ and the in-plane rotational $(\alpha)$ transformations. To compare two images, one needs to define the cross correlate coefficient (CCC) for each combination of the before mentioned transformations (Figure 2.7a).

The transformation matrix $G$, containing all three parameters $(x, y, \alpha)$, can be multiplied with the image to perform the transformation:

$$
G=\left(\begin{array}{cccc}
\cos \alpha & -\sin \alpha & x & 0 \\
\sin \alpha & \cos \alpha & y & 0 \\
0 & 0 & 1 & 0 \\
0 & 0 & 0 & 1
\end{array}\right)
$$

The euclidean distance between two images is a measure for similarity. By modifying x,y and alpha one aims to minimise the this distance between two images. The euclidean distance between the image $f_{2}\left(r_{i}\right)$ and the reference image $f_{1}\left(r_{i}\right)$, with $\mathrm{N}$ pixels and a grey-value $r$, can be minimised by multiplying the image $f_{2}\left(r_{i}\right)$ with the matrix $G$. This is shown exemplary only for translational transformations:

$$
\sum_{i=1}^{N}\left|f_{1}\left(r_{i}\right)-f_{2}\left(G r_{i}\right)\right| \rightarrow \min
$$

\footnotetext{
${ }^{15}$ Actually, only if fixed perpendicular translations and rotations are defined, three parameters are needed. In case that the second parameter is free, any position can be defined already by two transformations.
} 
The euclidean distance can be calculated as the cross correlation coefficient (CCC) between two images. The cross correlation function $(\mathrm{CCF})$ of two images $f_{1}$ and $f_{2}$ with $\mathrm{N}$ pixel is defined as:

$$
\begin{gathered}
{\left[f_{1}\left(r_{i}\right) ; i=1 \ldots, N\right] \text { and }\left[f_{2}\left(r_{i}\right) ; i=1 \ldots, N\right]} \\
C C C=\frac{\sum_{i=1}^{N}\left[f_{1}\left(r_{i}\right)-\left\langle f_{1}\right\rangle\right]\left[f_{2}\left(r_{i}\right)-\left\langle f_{2}\right\rangle\right]}{\sqrt{\sum_{i=1}^{N}\left[f_{1}\left(r_{i}\right)-\left\langle f_{1}\right\rangle\right]^{2} \sum_{i=1}^{N}\left[f_{2}\left(r_{i}\right)-\left\langle f_{2}\right\rangle\right]^{2}}} \quad \text { where }\langle f\rangle=1 / N \sum_{i=1}^{N} f\left(r_{i}\right)
\end{gathered}
$$

A high CCC value represents a high similarity between the images (compare Figure 2.7a). Here, the denominator term ensures the normalisation of the two images. The calculated CCC is a solution of the Cross Correlation Function (CCF). Mathematically, this is equal to the convolution of the particle image with the mirrored reference image. The Fourier transform of the convolution of two functions can be expressed as the multiplication of their individual Fourier transforms. The defined displacement of the correlation peak from the origin represents the shift of one image with respect to the other image:

$$
C C F(\vec{R})=f_{1}(\vec{R}) \star f_{2}(-\vec{R})=F^{-1}\left(F\left(f_{1}(\vec{R})\right) \cdot F\left(f_{2}(-\vec{R})\right)\right),
$$

where $F$ is the Fourier transform, $F^{-1}$ the inverse Fourier transform, $f_{1}$ the reference image and $f_{2}$ the image to be aligned. $\vec{R}$ defines a factor in Fourier space. More details about the mathematics of alignment can be found in Frank (2006, Chapter 3.3).

\subsubsection{References in Image Alignment}

The CCC is very sensitive to low SNR, and alignment can easily be biased by the used reference. Therefore, the choice of an appropriate reference is mandatory. A set of images of random noise can be aligned with any reference in a way that, when summed up, the respective reference is reproduced. Moreover, small differences in pixelsize between reference and image will bias the alignment. Usually, the reference is generated from the input data in a process called "reference-free alignment"16. By generating a rotational sum, the images are mass-centred, and thereby first class averages can be obtained as a set-up for a new, more precise alignment. The best defined class averages are selected and used as reference for the subsequent alignment. Accordingly, several references can be applied on one dataset, in a so-called "multi-reference alignment", where each image is aligned

\footnotetext{
${ }^{16}$ Originally termed "alignment via classification"
} 
to the reference of highest CCC (van Heel and Stöffler-Meilicke, 1985). Every rotational alignment step results in image interpolation and a corresponding loss of information. Therefore, as input for every alignment step, the original images are used to prevent information decay by interpolation.

A complete evaluation, where each possible combination of image shifts and rotations ${ }^{17}$ is compared by CCC with the reference, is called exhaustive alignment (Joyeux and Penczek, 2002; Sander et al., 2003). As alignment is a very calculation intensive process ${ }^{18}$ different simplifications are possible:

In the so-called direct alignment (M and H, 1980; Harauz et al., 1988), rotational and translational alignment are separated and alternated 2-5 times, until the image position does not change any more. Thereby the process becomes faster with still reasonable results. In multi-reference alignment, the restriction of one image to an earlier defined best reference can biase further alignment iterations, while the repetitive recalculation of the CCCs to all reference is very inefficient. By so-called corrim-based alignment, the calculations can be restricted to references with high correlation depending on a threshold defined by the user. This is less biased than restricting one image to one reference and faster than an exhaustive approach (Sander et al., 2003). Exhaustive alignment with resampling to polar coordinates shows an improved alignment quality when dealing with low SNR and can be accelerated in combination with the corrim-based approach (Sander et al., 2003).

Much progress has been made additionally by applying maximum likelihood (ML) methods to image alignment (Scheres et al., 2005). In this approach, each possible transformation will be weighted with a probability term, and the final transformation depends on the weighted best transformations. Similarly, ML approaches can be used for multi-reference alignment, where a weighted probability is assigned to each reference image. ML approaches are implemented in SIMPLE Prime and RELION (Elmlund et al., 2013; Scheres, 2012a,b).

\subsubsection{Classification of Images}

Aligned images can be classified independent from any given reference, depending only on internal image-specific features.(Figure 2.7b). However, there are computational limitations. Classification of $N$ images with $i^{2}=J$ pixels is impossible in reasonable time for datasets typical in single particle EM, with e.g. $N \geq 20000$ and $J \geq 4096$. The problem size needs to be reduced to a minimum, still describing the main image features. Images are

\footnotetext{
${ }^{17}$ The angular spacing defines the precision of the in-plane rotation. The minimum reasonable spacing is defined by the number of pixels on the outmost circumference of the particle.

${ }^{18}$ The calculation time is proportional to the square of the number of aligned images.
} 


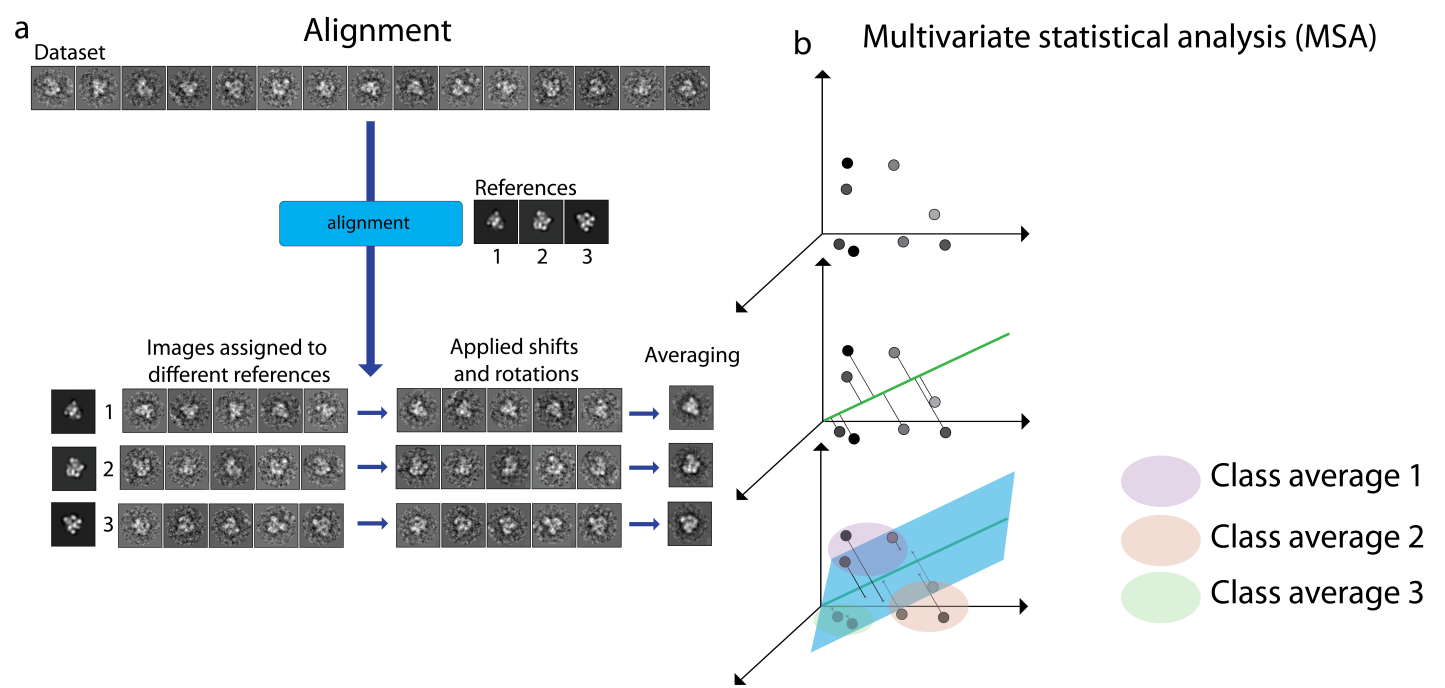

Figure 2.7: Alignment and Classification. a: A dataset composed of 15 images is aligned exemplary using three reference images (Multi-reference alignment). The images of the dataset are shifted and rotated to obtain the highest cross correlation coefficient (CCC). Each image of the dataset is transformed according to the reference image with the highest CCC. In this example, five images are related to each reference image. After shifts and rotation are applied, the images are averaged (Projection Matching). b: Multivariate statistical analysis (MSA) allows to classify images only based on internal image features. Images need to be aligned and can be subjected to MSA. In this example, 3-pixel-images are chosen and plotted in 3D space, where each axis ( $\mathrm{x}, \mathrm{y}, \mathrm{z})$ defines the grey value of one pixel. Accordingly, each dot represents one image within a data cloud of images. The main features of all images can be described by a vector pointing in the direction of the highest variance. A second perpendicular vector (not shown) describes the second most prominent feature. A set of those vectors (Eigenvectors) defines the similarity between images in order to be classified into distinct class averages (here in three sets: Class average 1, 2 and 3$)$. 
reduced to their key features, while random features of the images are omitted. The mathematical concept to reduce the problem size is based on "multivariate statistical analysis" (MSA) (Borland and van Heel, 1990; van Heel et al., 2009), while specifically the MSA method "principal component analysis" (PCA) will be explained here. Images are evaluated as vectors in $J$-dimensional space, the so-called hyperspace (Figure $2.7 \mathrm{~b}$ ). Accordingly, a set of images forms a cloud in hyperspace. Exemplary, if the images differ only in one specific pixel, the corresponding vector endpoints lie on a line parallel to the corresponding axis, defining that pixel. To express the similarity within the image cloud, another coordinate system is useful. A vector pointing in the direction of the biggest variance describes the main feature, the principal component, of all images. A perpendicular second vector, pointing in the direction of the second biggest variance, defines the second most contributing feature within the image set. This process can be repeated several times until only minor features are defined by the specific vectors. Accordingly, images can be transformed into a new hyperspace with these vectors as coordinate axis. These vectors are so-called "Eigenvectors" and the scalar products of these vectors are the corresponding "Eigenvalues". Every image within the image set can be expressed by a linear combination of scaled Eigenvectors. Usually, only a few Eigenvectors are necessary to define an image sufficiently. Thus, the data amount can be reduced to a smaller size by taking only the main Eigenvectors into account. Images can easily be compared and grouped according to their vectors in the new coordinate system. As the Eigenvector can be depicted as an image, evaluation of those Eigenimages helps to limit the data to the most prominent features.

Exemplary, a point cloud is given in a 2D coordinate system, describing images of two pixels by the vector $\vec{x}=\bar{O} \bar{P}_{i}$. A vector $\vec{u}$ needs to be defined for which the sum of the squared projections of all vectors is maximum. Thus, the vector points in the direction of the largest variance:

$$
\sum_{i=1}^{N} \bar{O} \bar{P}_{i}^{2}=\sum_{i=1}^{N} \bar{x}_{i} \vec{u}^{2} \rightarrow \max
$$

The whole image dataset can be expressed in a matrix, where each line contains a specific image and each row a specific pixel position:

$$
X=\left(\begin{array}{cccccc}
x_{11} & x_{12} & \cdot & \cdot & \cdot & X_{1 J} \\
x_{21} & x_{22} & \cdot & \cdot & \cdot & X_{2 J} \\
\cdot & \cdot & \cdot & \cdot & \cdot & \cdot \\
\cdot & \cdot & \cdot & \cdot & \cdot & \cdot \\
\cdot & \cdot & \cdot & \cdot & \cdot & \cdot \\
x_{N 1} & x_{N 2} & \cdot & \cdot & \cdot & X_{N J}
\end{array}\right) .
$$


By using the Eigenvector-eigenvalue equation, the problem defined in (2.38) can be addressed:

$$
D \vec{u}=\lambda \vec{u}
$$

where $\mathrm{D}$ defines the covariance matrix and $\lambda$ the Eigenvalue of the Eigenimage $\vec{u}$. The covariance of a grey-value $x_{j}$ of two different images $i$ and $i^{\prime}$ is defined by $d_{i i^{\prime}}$ for every image position:

$$
d_{i i^{\prime}}=\sum_{j=1}^{N}\left(x_{i j}-\bar{x}_{i}\right)\left(x_{i^{\prime} j}-\bar{x}_{i}^{\prime}\right)
$$

Computationally, this is solved by starting with random Eigenvectors, while by several rounds of multiplication with the covariance matrix of an image set, the resulting vectors will converge towards the real Eigenvectors (Borland and van Heel, 1990; van Heel et al., 2009). Each image can be expressed by a linear combination of scaled Eigenimages, where $c_{n}$ is a linear scaling factor:

$$
\vec{r}=c_{1} \overrightarrow{u_{1}}+c_{2} \overrightarrow{u_{2}}+c_{3} \overrightarrow{u_{3}}+\ldots
$$

By selecting the main Eigenvectors, the data can be reduced dramatically, while usually less than 50 Eigenvectors are sufficient to describe an image.

After data reduction, images need to be sorted into classes according to their similarity. There are different parameters to assign similarity, like the Euclidean distance, the chi-square metrics $(X)$ or the modulation distances (Frank, 2006; Orlova and Saibil, 2011). Principally, the smaller a value for the distance, the more similar the images, while, in detail, the result differs for the different parameters. Depending on internal normalisation, chi-square metrics $(X)$ or modulation distances are more robust to correctly sort images.

Independent of the used parameters, different procedures for classification can be used, like k-means and hierarchical clustering (Frank, 1990). In k-means clustering, a stochastic approach with randomly selected starting seeds is used, where images will be assigned to the nearest seed. The seed's position will be redefined iteratively to minimise the intra-cluster distance. This process performs best for small numbers of clusters and the result depends very much on the initial seed selection. Therefore, by multiple iterations of seed selection, an improved classification may be achieved. Also, k-means clustering can be combined with maximum-likelihood approaches. Hierarchical clustering starts either with a merged cluster for all images (hierarchical descendant classification) or by an individual 
cluster for each image (hierarchical ascendant classification). Then, depending on the distances between images, classes are separated or merged into new classes. Thereby, hierarchically, images can be sorted and their distance defines if they end up in similar or in different classes. This algorithm is successfully used in many image processing softwares like, Imagic and Spider (van Heel et al., 1996; Frank et al., 1981). In Imagic, the intraclass distance is iteratively minimised, while the interclass distance is maximised.

Classification is used either if no reference is given a priori in the so-called reference-free alignment approach or after alignment with a given reference to reduce the reference bias. Starting from a wrong reference will bias the classification result as the shifts $(x, y)$ and rotations $(\alpha)$ of any previous alignment steps influence the classification. Several rounds of alignment and classification will be needed to rescue the data from any introduced reference bias (Figure 2.9). In general, the user evaluates the class average and the similarity of the summed images and decides, which class average will be useful as reference for a following alignment step. In order to increase the chance to generate class averages representing all different projections, the number of classes is iteratively increased, with a reduced number of images per class, until reaching, by means of SNR, a minimum reasonable image set.

\subsubsection{Iterative Process}

The process of alternating classification and alignment is iterated, while the number of images per class is reduced, filter and alignment parameters are changed and the downsampling factor is stepwise decreased (meaning an increase of the applied pixel size) until no furhter optimisation of the $2 \mathrm{D}$ class averages can be observed. In this section, some general aspects to be considered are summarised (Figure 2.8).

Starting from a reference-free alignment in the first round, the number of classes is stepwise increased from a single rotational average. By increasing the number of classes, the number of images per class is reduced to $\sim 50$ images per class, while the increased number of generated classes, should allow further particle features to emerge. Class averages are selected manually, normalised and contoured as reference for the subsequent round of alignment. The selection should be based on the idea to include as many distinct features in the reference images as possible, while appropriate class averages are defined by high contrast, emerging details reduced blurred elements. Moreover, dot-like and ring-like features are likely to be biased and possibly need to be excluded. To be consistent in the processing, it is mandatory to compare and evaluate the class averages of the preceding iteration. Furthermore, the selection of class averages should be based on the selection of the proceeding iteration, while more or better class averages can be included. Otherwise, one may end of in an infinite loop. To prevent those drawbacks, class averages can be 
compared objectively with the output of the prior iteration by alignment and sorting of class averages, according to CCC or other measures of similarity (PCA or pHash), as manual evaluation is easily biased by "wishful interpretation". Comparing carefully the output of several iterations of classification should allow to distinguish real features from artefacts. It is reasonable to compare the class average image with the included individual images. If the superimposed image is very different from the individual images, the class average is not expected to be credible. The amount of heterogeneity can be estimated from individual images of a certain class, and potential local heterogeneity (representing local conformational changes) may be identified. Identified local heterogeneity allows to sort or split a dataset using corresponding references. The credibility of the alignment can be estimated by checking the shift and rotations of individual images in consecutive iterations. In a reliable alignment, $x, y$ and $\alpha$ should be consistent with the values of previous alignments, while a massive random walk of these parameters is a sign for alignment inconsistency. Only small shifts and rotations should occur, when the rough alignment is already stabilised. Furthermore, it is helpful to trace the CCC histogram of the overall alignment or of specific class averages or images. An improving alignment quality should correlate with improving CCC, until saturation is reached. Finally, it can be monitored how reliable images belong to a specific reference. Many images may "bounce" between several references with low CCC and can be excluded. They may still be of use, after a good starting model is defined, as they may represent rare projection images. Classification enables the identification of rare views and possibly of different conformations, especially if no 3D information is given. However, in very advanced projects with validated 3D volumes, the faster projection matching approach may be used.

\subsubsection{Projection Matching and Iterative Refinement}

In a process called "projection matching" (Figure 2.8), the angular information of the reference set is applied onto the corresponding image averages. After alignment, all aligned images are superimposed corresponding to the particular reference. 3D reconstruction is performed by applying the euler angles of the projected references onto corresponding image averages. The integrated images are used in a weighted back-projected approach to generate a 3D model. The 3D model is projected again and the projection images are used as new reference. Projection matching can be very convenient in specific cases. Projection matching is useful in cases where the binding site of a factor to a macromolecular complex of known structure should be identified. ${ }^{19}$. Furthermore, projection matching is applied to increase the resolution of an already validated but low-resolution model in a process called "refinement". In a "multi-model-multi-reference approach" a dataset can be sorted

\footnotetext{
${ }^{19}$ In this case, the mass contribution of this additional factor should be much smaller than the mass of the reference, as otherwise the alignment will be influenced.
} 


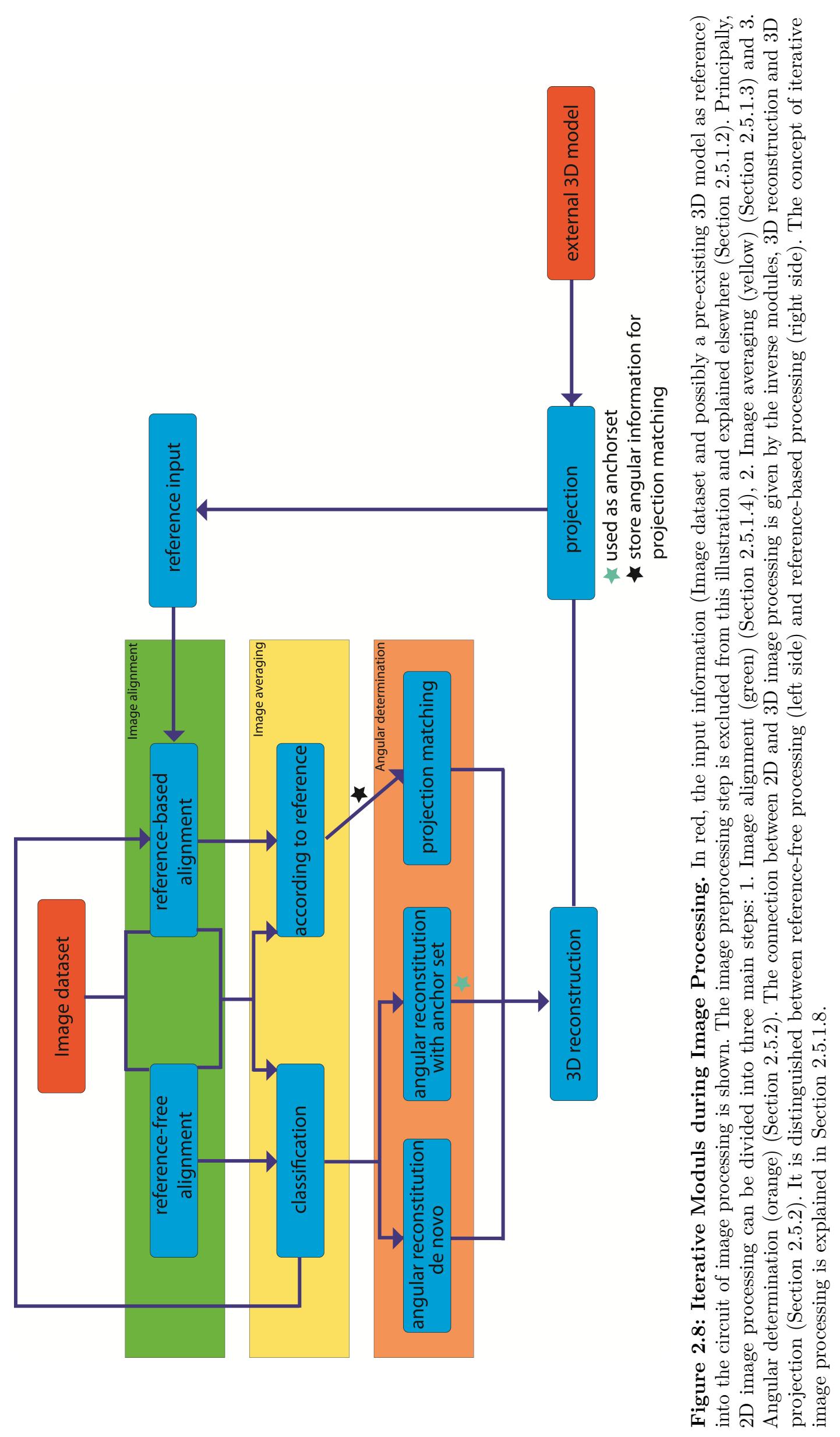


according to distinct reference models. As the angular information is provided only by the references and depends on the alignment quality, projection matching can be strongly biased towards the reference. Thus, this approach should only be used if a validated model already exists, as it can result in a mere reproduction of the used reference. The input reference may be too far away from the correct model to be correctly refined (e.g. by induced global conformational changes). However, with careful handling and validation, projection matching is very useful in any data refinement. Projection matching can be performed in real and reciprocal space, while the latter one is faster.

During initial processing, a 3D model can be improved by iterative classification and angular reconstitution. In the late state, the model is refined by projection matching, with the intention to improve the resolution of an initial model. There are several distict implementations of refinement available, using deterministic and maximum-likelihood approaches (Grigorieff, 2007; Scheres, 2012b). In general, refinement is characterised by repetitive iterations of alignment while monitoring the convergence of the data by different statistics. Fourier-Shell-Correlation (FSC) can be used to access the resolution of the obtained model. If the resolution does not improve any more, higher spatial frequencies can be included by changing the parameters like image filter and downsampling factor. In cases were heterogeneity is the limiting factor in resolution, refinement can be combined with excessive sorting either prior to refinement or by supervised classification during refinement. To speed-up the process, a restricted image shift and rotation during refinement is necessary, as is it utilised by corrim-based alignment (Sander et al., 2003).

\subsubsection{Statistics in Image Processing}

In 1995, the impact of radiation damage on atomic resolution reconstruction was evaluated (Henderson, 1995). The theoretical necessary number of particle images to yield a certain resolution level under perfect conditions was estimated. A relation was derived, where the number of images $N_{i m g}$ needed for the reconstruction of a 3D model is:

$$
N_{i m g}=\frac{300 \pi}{N_{e} \delta_{e} d}
$$

where $N_{e}$ is the absolute number of electrons per image, $\delta_{e}=0.005 \AA^{2}$ is the elastic cross-section for carbon and $d$ is the resolution.

Interestingly, it was shown that the expectable number of images for a certain resolution was independent of the molecular weight of the particle. In theory, 12600 images are needed for a resolution of $3 \AA$, while in case of $\sim 3800$ and $\sim 1900$ images, a resolution of $10 \AA$ and $20 \AA$, respectively, is still possible. 
In reality, images are not perfect and, due to low SNR and the molecular weight of the particle, the alignment quality and therefore the expectable resolution may be limited. In practice, datasets are usually much bigger and reach levels of more than $10^{6}$ images for atomic resolution. This depends mainly on the necessity of computational sorting steps. Not every image contains data of the same quality and not every particle is imaged in the same conformational state. In many cases, a huge image dataset needs to be sorted into smaller image sets to reach atomic resolution (Henderson, 1995). As already shown in Section 2.2.7 the applied electron acceleration voltage is relevant for the expectable $\mathrm{SNR}^{20}$.

\subsubsection{From 2D to 3D Level}

If reliable superimposed 2D images are obtained, they are used to reconstruct a 3D model. Different from projection matching, where the angular information is derived from the most similar reference image, angular information can be derived from 2D images only in a process called "angular reconstitution" 2.5.2.1 (Figure 2.8). Alternatively, by tilting the specimen in the microscope, images with given angular relationship can be obtained 2.5.2.2. Such a set of so-called tilted image pairs can be used to reconstruct a 3D model.

\subsubsection{Angular Reconstitution and 3D Reconstruction}

The mathematical relationship between projections and a 3D model is defined by the Fourier slice theorem (Bracewell, 1956). Each Fourier transform of a 2D projection resembles a central section of the Fourier transform of the underlying 3D object. Therefore, two 2D projections share in Fourier space a central section.

In principal, angular assignment can be performed in real and in Fourier space. Here, the real space approach of IMAGIC and cowEyes is explained. In real space, this is performed by using so-called common lines (Van Heel, 1987). Common lines are all possible central $1 \mathrm{D}$ projections of a 2D image. If two projection images are related and consequently share a central section in Fourier space, they share a common line in real space. The principal projection transformation is the Radon transform:

$$
R f(r, \alpha)=\int_{-\infty}^{+\infty} f(r \cos \alpha+t \sin \alpha, r \sin \alpha-t \cos \alpha) d t
$$

where $r$ defines the distance from the origin and $\alpha$ the direction angle of the projection. Thereby, the function $f(x, y)$ can be projected in every wanted direction. Using the Radon

\footnotetext{
${ }^{20}$ These calculations are not true for negative stain processing, where the SNR of low-spatial frequencies is much higher. The expectable resolution is mostly limited to $\sim 10-25 \AA$.
} 
transform, a table can be defined containing in each row one 1D projection, the so-called sinogram, while for every projection, $\alpha$ is changed. Thus, a line-by-line representation of all central projections is obtained. Two images can be compared by their sinograms. If they are derived from a common 3D object, they share, at a certain angle $\alpha$, a common 1D projection, the common line. Common lines between sinograms can be defined by cross correlation in real space or by forming a summed conjugate product in Fourier space. The angular relationship between two projections is defined by $\alpha$. However, without additional information, the central section can still rotate around the common line. Therefore, a third projection with a different tilt axis is used to define a fixed relation of the three projections in 3D space. Basically, using these three perpendicular projections, all information is given for the definition of the Euler angles and consequently for the reconstruction of the initial 3D model. Due to image imperfection, in practice there is more then one possbible solutions for the correlation of two sinograms. Thus, many class averages are combined for angular reconstitution. Moreover, by substitution of individual class averages in a class average set used for angular reconstitution, angular errors, resulting from wrongly assigned class averages, can be reduced. If a particle contains any internal symmetry, this symmetry can be used for angular reconstitution (so-called self-common lines), making the angular assignment more robust, and less class averages are needed. As in 1D projections the information is very condensed, a voting algorithm helps to define the correct solution in cases which are not unambiguous (Singer et al., 2010).

The accuracy of the angular reconstitution can be improved if a 3D model is given. The projections of this 3D model can be used to calculate sinograms, and instead of correlating sinograms between differnt class averages, sinograms between the projection of the 3D model and the class averages are calculated. In this case the projections are used as so-called "anchor set" (Figure 2.8). In the case of a correct 3D model, this approach performs better than a de novo angular reconstitution, due to improved SNRs and a higher angular integrity.

The 3D reconstruction of projection images can be performed in real space and Fourier space. However, a simple inverse Radon-transform is not useful in structural biology. Also, a back-projection generates a 3D model, which is smeared out in the direction of projection, due to the convolution of each image with a Point spread function (PSF). By so-called filtered back-projection, this problem is addressed by applying a filter which is inverse to the PSF. Furthermore, in real space, algebraic methods derived from medical tomography and simultaneous iterative reconstruction exist. In Fourier space, Fourier inversions and Bessel reconstruction are used (reviewed in Orlova and Saibil, 2011). 


\subsubsection{Random Conical Tilt Reconstruction}

Besides the in silico determination of the angular relationship of projection images, an experimental approach is possible. This is based on the option to tilt the specimen within the specimen holder in the microscope. In this case, the angular information is not derived mathematically from related projection images but experimentally by recording the same object twice with distinct tilt angles. The most common approach is based on so-called random-conical tilting (RCT) (Radermacher et al., 1987). Images are recorded twice, once without tilting $\left(0^{\circ}\right)$ and once with tilting the sample holder $\left(45-60^{\circ}\right)$. Tilting an image results in a translational shift $(\mathrm{X}, \mathrm{Y})$ and a changed in-plane rotation $(\alpha)$. Additionally, three random points will change their apparent distance and angular relationship within the projection. This information can be used to computationally validate the experimental tilt angle (tilt angle and in-plane rotation $\alpha$ ). Identical particles of the non-tilted and tilted micrographs are indexed as pairs to backtrace this relationship throughout image processing. Usually, only a few thousand images are required. The non-tilted images are processed until they cluster into reliable $2 \mathrm{D}$ class averages. Thus, several non-tilted images are superimposed in a class average, while the index between non-tilted and tilted images allows to relate tilted images to certain classes. Due to the random in plane rotation of the particle, the tilted images are arrayed on a cone relative to the central class average of their non-tilted counterpart. Thus, by filtered back-projection, an individual 3D model can be derived for each class average combining the tilted and non-tilted information. Each 3D model will suffer from a missing cone in Fourier space, depending on the chosen tilt angle. In real space, the 3D model is distorted in a certain direction. The direction of distortion changes for different 3D models, so that, by 3D alignment and 3D MSA with subsequent classification an improved 3D model can be calculated, which can be used as starting model for further image processing.

There are some limitations related to the RCT approach. First of all, the same position is imaged twice, resulting in accumulated beam damage in the second image. Usually the tilted image is recorded first, as a higher image quality is helpful for alignment and classification. Still, in cryo condition the information content of the second image is lowered due to double exposure, especially concerning higher spatial frequency. Another crucial limitation is the tilting of the specimen itself. In cryo conditions, tilting can result in severe charging effects. In certain directions, Thon rings are missing, and therefore, only very low-spatial frequencies are available. Moreover the contrast of tilted images is lower. Beam damage is less limiting if negatively stained particles are used, but other artefacts become limiting in negative stain. Homogeneous staining is a prerequisite for high image quality, as otherwise, at the border of particles, dark high-contrast fringes become visible. These fringes result from distinct stain thickness and limit the alignment performance. 
By increasing the tilt angle, this effect is increased and the very small changes in stain thickness lower the quality of the image dramatically. A different approach is orthogonal tilting (Leschziner and Nogales, 2006), where two tilted images, one at $-45^{\circ}$ and the other at $45^{\circ}$ are recorded. Thereby, the missing cone is eliminated, but the limitations due to higher tilt angles is increased. For small particles $(\leq 500 \mathrm{kDa})$, all available tilt techniques are of limited performance. In general, the obtained 3D models need to be tested carefully.

\subsubsection{Initial Model Building}

Initial model building can be very tricky, especially for small, heterogeneous and asymmetric particles. Here, some ways to allow initial model building are introduced. Even so, on 2D level, promising class averages are gained, sometimes neither angular reconstitution nor RCT perform good enough to obtain a reliable 3D model.

In cases where no angular convergence of generated 3D models becomes obvious, the angular relationship can be addressed by statistic approaches, so-called "resampling". By $n$ times randomly drawing $m$ class averages from a set of class averages (with the set size l) for angular reconstitution, $n$ 3D models can be generated. Principally, this process is a bootstrapping approach (Penczek et al., 2006). The number of calculated sets $n$ and the number of class averages per selected set $m$ can be specified by the user. The dragging of individual class averages can be modified to chose between pure random selection, selection of very distinct class averages or selection of class averages with very different pre-determined euler angles. Thereby, a set of 3D models can be generated, which can be aligned and classified by 3D MSA. Most of the gained 3D models will be deficient. However, using these high statistics, reasonable 3D models may be generated and can be recognised as possible starting points for further image processing. The necessary statistics can easily be calculated by:

$$
n=\frac{m ! \cdot l_{1} \cdot l_{2} \cdot \cdot \cdot l_{n}}{l_{n}}
$$

Another method to overcome the limitation of initial model building is based on the maximum-likelihood approach, implemented in the free software suite SIMPLE, a probabilistic projection matching algorithm (Elmlund and Elmlund, 2012). Usually, the angular information of images is attributed in an deterministic way. In projection matching, angular information is assigned according to best-scored reference and the according angular information is applied for reconstruction. In a probabilistic approach, several possible solutions are tolerated in parallel, while a weighting factor determines the contribution of each possible solution, according to the correlation to a reference image. Thereby, 
iteratively, the probabilities may change, allowing an initial model to converge into an improved direction. This is important for the model building as the highest score does not need to be the correct one. Other solutions are included, which may outvote the initial highest scoring during refinement. In a deterministic approach, a model cannot recover from a wrong local minimum defined by a determination to an initial high but wrong scoring. In SIMPLE Prime, an initial random model is generated and correlated to the input images. The highest scoring correlations are included in a weighted projection matching approach, to reconstruct a new model which can be projected and compared with the input images. Correspondingly, the output model can be different in several runs, depending on the chosen initial seeds. The algorithm was shown to be successful in obtaining an initial model with 70S ribosomes. However, in cases of small heterogeneous datasets, 2D processing to generate class averages can be applied first to increase the SNR. The set of class averages can be used in SIMPLE Prime, similar to an initial image set but with improved performance due to better SNR. Additionally, as only 100-200 class averages are used instead of 10,000 images, the process is accelerated.

\subsubsection{Angular Distribution}

The angular distribution, which is assigned for individual images or class averages can be plotted in a 2D map or a spheric map on top of the 3D model. The evaluation of the angular distribution is useful to define the angular coverage, while missing views result in lower resolution in certain projection directions, so-called anisotropic resolution. Class averages, where no confident angular assignment is possible, can be omitted to improve the quality of the 3D model. Secondly, missing views can be included by either chemically changing the binding behaviour of the particle on the carbon film or by including low tilt angles during image recording (e.g. $15^{\circ}$ ). Both procedures can result in improved angular coverage and in improved 3D models. The chosen angular sampling, affects the reachable resolution of a $3 \mathrm{D}$ map. In case of projection matching the maximum resolution $s_{\min }$ depends on the angular distance of the used projections:

$$
s_{\min }=d \sin ^{2}\left(\frac{\phi}{2}\right)=\frac{2 d}{n}
$$

with the particle diameter $d$, the angular spacing of projections $\phi$ and the number of projections $n$ (Harauz and van Heel, 1986). For an angular reconstitution approach no even angular coverage is given and therefore no simple formula can be defined. 
Figure 2.9: Model Bias can be induced by a Wrong Reference Models. Exemplary, a 3D reference model of a dinosaur is applied on a dataset of Pds5 - bonsai cohesin tetramer to show the effect of model bias. The danger of model bias becomes obvious in this illustration. Depending on the used approach, the introduced model bias can vary. By projection matching, a strong model bias is introduced, while an additional classification step reduces the model bias. However, several rounds of alignment and classification are necessary to regain the features of the dataset in the 3D model. The topic of erroneous 3D models by the application of wrong reference models or by working on pure noise images has been shown in related literature (Henderson, 2013; van Heel, 2013; Stewart and Grigorieff, 2004; Penczek et al., 1994).

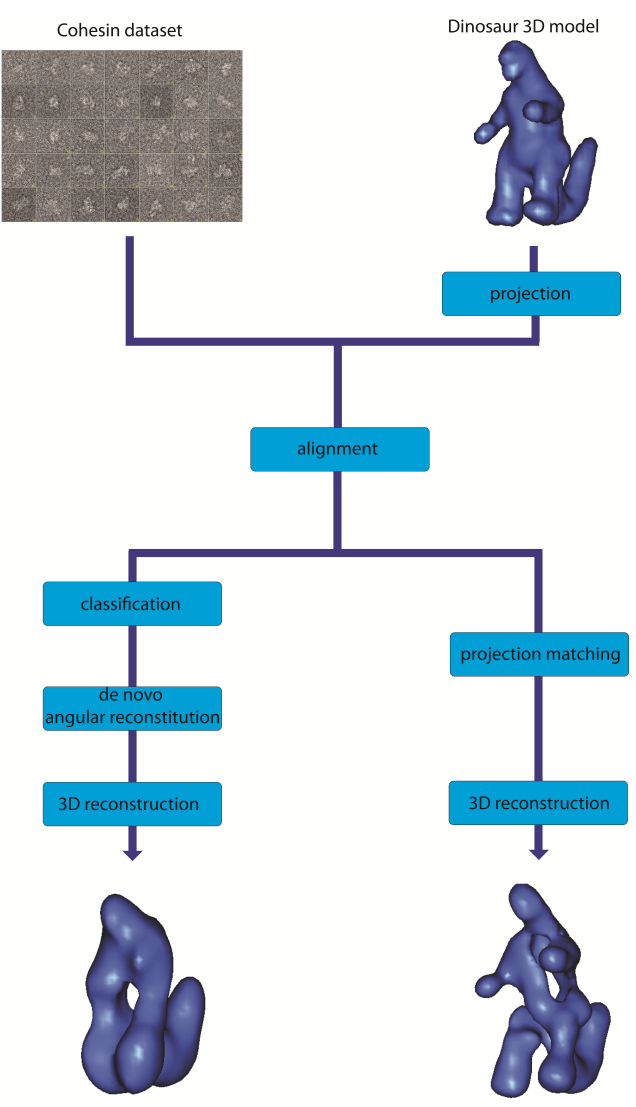

\subsubsection{Computational Strategies for Sample Heterogeneity}

As mentioned earlier, it can be distinguished between compositional and conformational heterogeneity within the sample. Both types of heterogeneity can affect the macromolecular complex from a very local level up to a global level. Additionally, one can distinguish between functional and non-functional heterogeneity (see Section 1.4.1).

Principally, heterogeneity can be addressed by sorting the datasets, according to their difference, into several subsets. All methods, which will be introduced in the following two sections can be used to perform this sorting step. In this context, it is important to point on the danger of model bias (Figure 2.9) (Henderson, 2013; van Heel, 2013; Stewart and Grigorieff, 2004; Penczek et al., 1994). A dataset divided according to a reference set by supervised classification or ML approaches has to be cross-validated. In an exemplary case with two distinct states, the following scenario holds true: If the divided subset 1 (assigned to reference state 1) and subset 2 (assigned to reference state 2) are refined with the corresponding other reference model, it should not be possible to regain this wrong reference model from that data. Otherwise, the specific feature depends to a larger extend on the used reference than on the sorted data, and the sorting is wrong. Accordingly, the sorting on this feature is artificial. The size of the required dataset scales with the number of states which are expected to be defined. Therefore, sophisticated sorting strategies have 
high requirement in terms of data-size. Often, given the limitations of each techniques, only a combination of different tools will allow to solve the addressed problem.

By hierarchical computational sorting it is possible to define many different states of the complex of interest at high resolution. Usually, this is started by sorting for global changes, while in later steps it can be sorted for more subtle heterogeneity. With available structural models, an single particle EM dataset can be sorted by so-called supervised classification (Gao et al., 2004). In this approach, several different 3D models are projected and used as reference set for alignment. Thereby, images are assigned to the best fitting model in a competitive way, and different models can be refined from one dataset. This procedure is of great success in ribosome research, answering questions concerning the $30 \mathrm{~S}$ ratcheting, tRNA dynamics and factor binding (Fischer et al., 2010). However, the method is always limited by the necessity of specific references (Henderson, 2013; van Heel, 2013; Stewart and Grigorieff, 2004; Penczek et al., 1994) and should be only used if the expected dynamics are at least party understood. A reference can even be modelled, based on prior knowledge, but this needs to be validated by different techniques to minimise model bias.

If the global structure is less heterogeneous but locally structural changes are to be expected, this area can be subjected to 3D multivariate statistical analysis (3D MSA) and subsequent classification. This allows to sort similar 3D volumes into distinct classes and obtain 3D models of different conformations. The combination of a bootstrapping approach (Penczek et al., 2006) with 3D MSA and subsequent classification is powerful and less biased than supervised classification. The bootstrapping approach is performed by randomly selecting particle images or class averages for the generation of initial 3D volumes, so-called "resampling" (see Section 2.5.2.3). These initial 3D models are classified according to similarity and refined. It is helpful to proceed in a stepwise approach, from bigger to smaller conformational changes (Fischer et al., 2010). If satisfying statistics of the different states are given, many states can be resolved. A successful example is given in case of the 70S ribosome (Fischer et al., 2010). However, the process is computationally very demanding. In Section 3.2.3 a similar sorting strategy is described. Different from that, there are 3D maximum-likelihood approaches, where no prior structural knowledge is needed, so-called unsupervised refinement. In this procedure, an initial reference map is used to generate a predefined number of low-resolution models from randomly drawn subsets of the data. These models are the starting points for competitive refinement, where, different from conventional projection matching, all assignments are generated in a probability-weighted routine. Continually higher resolution information is integrated into the iteration. Again, stepwise, starting from an initial model, the dataset can be classified into subsets, lowering the variance within one population. Maximum-likelihood (ML) approaches are implemented in software like SIMPLE Prime and the 3D classification of RELION (Elmlund et al., 2013; 
Scheres, 2012a,b). Principally, both can perform heterogeneity analysis, while RELION was already shown to be very useful for this purpose (e.g. Campbell et al., 2015). As the number of classes can be defined, the full range of dynamics can principally be analysed. However, the output is greatly influenced by the input model and the applied mask and shows limited performance for small particles. As stated earlier, Normal mode analysis (NMA) is an interesting tool to generate different 3D references on pseudo-atomic basis from EM maps (Lindahl et al., 2006; Nogales-Cadenas et al., 2013). While the chemical nature is ignored, dynamics are obtained by pure mechanic considerations. These references can be used as starting point for ML approaches or supervised classification. NMA can only help to develop ideas of real dynamics. Additionally, different variants of RCT can be used to define heterogeneity, as several sets of low-quality 3D models can be generated (Radermacher et al., 1987; Leschziner and Nogales, 2006). These 3D models can serve as input for ML approaches like RELION (Scheres, 2012a,b) or supervised classification (Gao et al., 2004). As already outlined, resolving a trustful 3D model of small particles is likely to fail using this approach.

\subsubsection{Resolution Assessment}

A 3D map is a density distribution, where based on the applied threshold a specific surface view can be defined. The threshold needs to be chosen adequately to distinguish biological features from noise. Moreover, the interpretable resolution of a 3D model needs to be measured.

In some cases, random noise mimics helical features or side chains and can be regarded misleadingly as structural information. This problem is described by the term "overfitting" ${ }^{21}$. In contrast, underfitting describes a state where not all data is used optimally and the derived model fails to optimally characterise a real state. Even more, the resolution and reliability of a structure can be disproportionate regarding different locations of a structure. In extreme cases, local overfitting is possible. This is termed "anisotropy" and can result from local sample heterogeneity.

A universal resolution criteria is necessary to address the above stated problem. Different resolution criteria were defined before:

1. The diffraction-limited Rayleigh resolution $d_{\min }($ see $(2.2)$ )

2. The $C_{S^{-}}$limited resolution $r_{\min }($ see $(2.13))$

3. The angular sampling-limited resolution $s_{\min }($ see $(2.46))$

\footnotetext{
${ }^{21}$ Overfitting is a problem often deriving from multi-parameter problems, where the number of uncoupled unknown parameters outmatches the known parameters to describe the model.
} 
4. The Nyquist-Shannon sampling theorem-limited resolution (see (2.5.1.2))

1. and 2. are related to electron optics, while 3. and 4. are related to image processing. However, if images are not downsampled the Nyquist frequency $f=\frac{1}{2 d}$ depends on the detector, specifically the pixel size at a certain magnification. The practical resolution limit is usually defined by the Nyquist limit.

To approximately estimate the resolution, one can compare the level of detail of a structure ( $\alpha$-helix, $\beta$-sheets) with a set of structures of given resolution. Most structures do not reach resolution levels where biological characteristics like individual side chains $(4 \AA)$, separated $\beta$-sheets ( $5 \AA$ ) or $\alpha$-helices (9 $\AA$ ) can be distinguished. Additionally, one can down-filter a corresponding crystal structure to have a similar appearance as the structure to estimate. In a more objective way, by "Fourier shell correlation" (FSC) the resolution of a 3D object can be determined mathematically. There, individual spatial frequencies of two 3D structures are correlated with each other (Harauz and van Heel, 1986; Penczek, 2010). Similarly, in 2D space the equivalent is the Fourier ring correlation (FRC) (Saxton and Baumeister, 1982). The correlation fall-off defines up to which limit a spatial frequency is still trustworthy. This can be evaluated with the spectral signal-to-noise-ratio (SSNR). Usually, the resolution is stated according to the 0.5 or 0.143 criterion, with a value of 0.5 corresponding to a SSNR of 1 . This means that half the visible details between two structures at a certain spatial frequency can be correlated. To handle anisotropy, local FSC can be performed. In this case, the correlation is defined for different commensurate regions independently. A suitable window size for an area of interest is chosen, and the local FSC between different areas of equivalent window size can be obtained.

Recently, in an international single particle EM meeting (Henderson et al., 2012), the gold standard refinement was postulated as a standard criterion for the single particle EM community. The gold standard criterion states that every dataset needs to be divided randomly in two equal sized parts and must be refined independently. Two independently derived structures are compared based on the SNR of the data they derived from.

Mathematically the FSC can be expressed as:

$$
F S C(k)=\frac{\sum_{k, \Delta k} F_{1}(k) \cdot \overline{F_{2}(k)}}{\sqrt{\sum_{k, \Delta k}\left|F_{1}(k)\right|^{2} \cdot \sum_{k, \Delta k}\left|F_{2}(k)\right|^{2}}},
$$

where $F_{1}(k)$ is the $3 \mathrm{D}$ structure derived from the first half dataset, and $F_{2}(k)$ is the $3 \mathrm{D}$ structure derived from the second half dataset, from which the complex conjugate is formed. The specific spatial frequency is determined by $k, \Delta k$. The $S S N R(k)$ relates to the $F S C(k)$ in the following way: 


$$
\operatorname{SSNR}(k)=\frac{F S C(k)}{1-F S C(k)}
$$

\subsubsection{Structural Validation}

In spite of the great achievements in atomic resolution cryo EM (better than $\sim 3 \AA$ ), many complexes are still limited to much lower resolutions. Especially, those structures demand an unbiased validation criterion. As already outlined, generating a new model of any macromolecular complex without prior knowledge is rather difficult and can easily end up in a wrong model. Thus, there is the necessity to evaluate the reliability of a structure. However, this is still difficult in many cases, and a generally approved standard needs still to be defined, which is the main reason for the Validation task force in 2012 (Henderson et al., 2012). Single particle EM is much younger than e.g. X-ray crystallography, where equivalent standards were defined in the 1990s. At that time, the first automated software packages for single-crystal structure validation, emerged. In early crystallography, one of the main criteria for structure evaluation was the R-value. From the derived model, a simulated diffraction pattern is calculated, the experimental and simulated diffraction patterns are compared, and similarity is expressed by the R-value. A random set generates an $\mathrm{R}$-value of $\sim 0.63$, while a perfect fit results in a value equal to 0 . However, typical data are in the range of $\sim 0.2$. Despite the fact that a model is never perfect (e.g. water channels, disorder and vibration), the R-factor has a conceptional problem as it is not free of bias. By improving a model to make it better fitting to the experimental data, the R-value is improved. In this case, the atomic model is used with the experimental diffraction pattern, and the self-sufficiency is lost. Accordingly, the R-free was introduced 1992 and is derived by removing $10 \%$ of the data. The refined model is used to define how good the model predicts the independent $10 \%$ of the data. In an ideal case the R-free is similar to the $\mathrm{R}$-value, in practice it is usually a little higher ( $\sim 0.26)$. In cryo EM, there is still the need to develop something similar to validate a structure. So far, two interesting concepts can be introduced, which are the tilt-pair analysis and the omit map recovery.

The tilt-pair analysis was introduced in 2003 as a tool to define the handedness of a structure (Henderson et al., 2011b). Due to the projection nature in TEM, the absolute handedness of any model cannot be defined without tilting experiments or independent structural data (e.g. X-ray data, MS, visible $\alpha$-helical twist). Tilt-pair analysis is a powerful tool, very precise for big complexes ( $\sim 50 \mathrm{MDa})$ and still satisfying for complexes from 1 to $5 \mathrm{MDa}$, with small angular inaccuracies. However, for small complexes, it may fail as inaccuracy becomes to huge. Even more, heterogeneous datasets reduce the usability of this evaluation even more, so that, in many cases, validation fails, although the model 
is correct (See Section 2.5.2.2). Charging effects in vitrified samples and stain fringes in negative stain samples limit the applicability to small and heterogeneous particles.

In principal, the procedure is as follows: Non-tilted and tilted micrographs are recorded, while the tilt angle can be lower than in RCT $\left(20-45^{\circ}\right)$. A few hundred up to thousand tilt pairs are selected. The non-tilted images can be included in the image processing procedure of a larger dataset to obtain a 3D model. The tilt-pairs are aligned against reprojections of this 3D model. The rotational angle and the tilt angle are either known or calculated from the micrograph. The theoretical rotational angle and the tilt angle to transform the tilted data into the non-tilted data is calculated. This can be plotted in the so-called tilt-pair parameter plot (TPPP). If the data cloud clusters around the focal point of the experimental tilt angle, the model is correct. The size of the cluster defines the accuracy and the reliability of the validation. In a similar approach (Kirves, 2014), two 3D structures are calculated, one from the non-tilted data and one from the tiled data, and compared by rotational correlation. A valid model will show a higher correlation, while a specific validation factor indicates the validation quality. A value greater than 7 is trustworthy, while values below 6 argue for a insufficient model. First, non-tilted images are aligned against the projections of the reference 3D model. The defined Euler angles, the experimental tilt angle and in-plane rotation can be used to define Euler angles for the tilted images. Accordingly, the two 3D models are obtained. The non-tilted data is reconstructed by projection matching to the reference and the tilted data is reconstructed with the respective calculated Euler angles.

The second approach, the so-called omit map recovery, circumvents the difficulties related to image tilts. Here a reference model (here defined as $A$ ) is needed. A part of the structure is removed and the data is refined against the remaining part $B$. This process is repeated in a way that every part of the structure is once omitted. All reference-free parts of the distinct output 3D models are summed to yield a complete model $C$. In a valid structural model every omitted region should recover. The correlation between the reference model $A$ and the output model $C$ defines a parameter describing the correctness of the input model $A$. Again, this approach is limited by sample heterogeneity. Furthermore, the size-ratio and form of the removed region to the remaining region is relevant for the precision of the alignment. In practise, the 3D model is divided into 8 boxes with $\frac{7}{8}$ of the model used as reference. The box size has an effect on the reliability of this tool, as cutting only very small parts away (e.g. $\left.\frac{63}{64}\right)$ can result in false positives.

There are further ways to validate a structure. In the case different subcomplexes of a complex exist where specific subunits $(\mathrm{A}, \mathrm{B}, \mathrm{C}, \mathrm{D}, \mathrm{E})$ are exchanged or added, the according datasets can be processes in a reference-free procedure to generate independent 3D models. In the exemplary case of three different complexes, A-B-C-D (1), A-B-C-D-E (2) and A-B-C-E (3), the corresponding 3D models can be compared with each other. If their 
structure can be related while an additional density is identified in case (2) and (3) it is very likely, that the three models are correct, as it is unlikely that three similar but independently derived structures are wrong. However, this approach precludes strong conformational changes related to the subunit exchange. Additionally, the structure can be validated by fitting subunits (e.g. NMR or X-ray data) into the density and calculating the molecular weight of the segmented subunits. If the molecular weight fits the expected molecular weight of the corresponding density and if the subunits can be fitted properly, further hints for the correctness of the models are given. However, auto-segmentation and manual segmentation can be very biased and the enclosed volume strongly depends on the chosen density threshold. Also, fitting of crystal structures can be very biased and strongly depends on the resolution level. Before fitting, the corresponding crystal structure should be filtered to the resolution of the 3D model. In summary, segmentation and fitting need be handled carefully and is rarely a stand-alone tool for validation.

\subsection{Summary of Image Processing Procedure in this Work}

Images were selected using the in-house software CowPicker. There, a manual, a semiautomatic and automatic modus can be applied. For ribosomes, automated selection was possible. For the different cohesin complexes, due to their unknown structure and heterogeneity, a semi-automated particle selection was chosen. Image selection was performed in a 2-4 times particle diameter. Images were appended and stored as IMAGIC stack. CTF correction was performed with the in-house softwares CowEyes or with an in-house script powerspec_new.exe and CowEyes for classification. The powerspectra were processed by PCA and classification and PCTF parameters were defined. Manually, non-round classes (astigmatism), low resolution classes (defined by limited Thon ringe extension) and too low or high defocus classes were removed. Image stacks were cleaned by repetitive initial classification steps, to remove unwanted particles. In the case of the ribosome a supervised classification with a $50 \mathrm{~S}$ reference and $70 \mathrm{~S}$ reference was used to remove undesired free 50S subunits. Initial processing was performed as described previously (2.5.1.2). A twofold downsampling factor was applied and bandpass filtering parameters were calculated according to Formula 2.31. Thereafter, the images were normalised. 2D and 3D processing of ribosome and cohesin was performed by various tools, including CowEyes, Imagic, SIMPLE and RELION and scripts by Björn Sander. Details of specific steps are defined in the related sections above (2.5.1 and 2.5.2). Image processing resulted in several interim models, which are used as reference for further processing. Initial model building for different cohesin complexes was performed by SIMPLE Prime (Elmlund et al., 
2013) or by a bootstrapping approach (Penczek et al., 2006) (see 2.5.2.3). Conformational sorting was applied by supervised or by unsupervised classification approaches, or by 3D MSA. Unsupervised classification, was possible by 3D-maximum likelihood classification in RELION (Scheres, 2012a,b). Structural assessment and manipulation was performed in Amira or Chimera. Fourier shell correlation was performed within CowEyes.

The $\mathrm{C}++$ in-house software cowEyes was used for most image processing steps. It provides an easy-to-use GUI, where different kind of processing modules can be connected. In principal, it is designed in a way that it can be used with LINUX and Windows systems. Thereby, a module network is generated, where all image processing steps can be easily accessed and are stored within a history file system. CowEyes can be used locally or on a server, depending on the requirements of the specific calculations which need to be performed. Calculations can be performed on GPU or CPU depending on the specific module and data requirements. Moreover, a $2 \mathrm{D}$ and $3 \mathrm{D}$ visualisation module is integrated.

RELION is a software (LINUX) for CTF processing, 2D classification, 3D classification and refinement. All processes can be easily performed via GUI or command line. The huge amount of automation makes the software easy to use and according to the developer does not need user expertise. Therefore, it became increasingly popular within the single particle EM community. However, user expertise is needed to evaluate the reliability of the generated models as in every available software. The software makes use of maximumlikelihood approaches to perform $2 \mathrm{D}$ and $3 \mathrm{D}$ classification. In this work, RELION was mainly used for 2D and 3D classification purposes. 


\subsection{Equipment and Chemicals}

\subsubsection{Special Equipment}

Table 2.6: Machines and Special Equipment

\begin{tabular}{l|l}
\hline Equipment & Manufacturer \\
\hline Centrifuge Avanti & Beckman Coulter \\
Centrifuge WX Ultra 90 & Sorvall \\
$C_{S}$ corrector & CEOS GmbH \\
Eagle 4k CCD Camera & FEI \\
Falcon2 CMOS & FEI \\
Gradient Master ip & Biocomp \\
Rotor TH660 & Sorvall \\
TEM CM200 FEG & Philips \\
TEM Titan Krios & FEI \\
TemCam F415 (4K CCD) & Tietz Video Systems \\
Vitrobot IV & FEI \\
\hline
\end{tabular}




\subsubsection{Chemicals}

Table 2.7: Used Chemicals

\begin{tabular}{l|l}
\hline Chemical & Supplier \\
\hline Aceton & Merck \\
Adenosinetriphosphate (ATP) & Amersham Biosciences \\
Bradford Assay reagent & BioRad \\
Dimethylsulphoxide (DMSO) & AppliChem \\
Ethane (liquid) & Messer \\
Ethanol & Merck \\
Glutaraldehyde & Science Services \\
Glycerol & Merck \\
4-(2-hydroxyethyl)-1-piperazineethanesulfonic acid & Sigma Aldrich \\
(HEPES) & \\
Iron(III) sulfate hydrate & Merck \\
Isopropyl $\beta$-D-1-thiogalactopyranoside $($ IPTG $)$ & Roth \\
L-Aspartate & Sigma Aldrich \\
Magnesium chloride & Merck \\
Magnesium sulfate & Merck \\
Magnesium sulfate & Merck \\
Millipore water & EMD Millipore \\
Potassium chloride & Merck \\
Potassiumdihydrogen phosphate $\left(\mathrm{KH}_{2} \mathrm{PO}_{4}\right)$ & Merck \\
Putrescine & Fluka-Riedel-de-Haen \\
Sodium chloride & Merck \\
Spermine & Fluka-Riedel-de-Haen \\
Spermidine & Fluka-Riedel-de-Haen \\
Sucrose & Merck \\
Uranyl formate & Polyscience Inc. \\
\hline
\end{tabular}


Table 2.8: Used Laboratory Materials

\begin{tabular}{l|l}
\hline Laboratory material & Supplier \\
\hline Balances & Sartorius \\
Filter paper $90 \mathrm{~mm}$ Whatman & GE Healthcare \\
Mica, $75 \times 25 \mathrm{~mm}$ & Plano \\
Nitrocellulose, $0.2 \mathrm{~m}$ & Whatman (GE Healthcare) \\
Parafilm M & Bemis Company \\
PD MiniTrap G25 columns & GE Healthcare \\
QUANTIFOIL grid R3.5/1 Cu 200 mesh & QUANTIFOIL Microtools \\
Sterile Filters $0.2 \mathrm{~m}$ & Millipore \\
Zeba spin desalting columns, 7K MWCO & Thermo Fisher Scienctific \\
\hline
\end{tabular}




\subsubsection{Software}

Table 2.9: Used Software

\begin{tabular}{l|l}
\hline Software & Source \\
\hline Amira 5.2.2 & Visualization Sciences Group \\
cowEyes & custom made at Stark lab \\
Imagic & $\begin{array}{l}\text { Image Science } \\
\text { custom made at Stark lab } \\
\text { cowPicker } \\
\text { Phttps://www.python.org/ } \\
\text { downloads/ } \\
\text { http://www2.mrc-lmb.cam.ac. } \\
\text { uk/relion } \\
\text { SIMPLE Prime } 1.3\end{array}$ \\
UCSF Chimera & $\begin{array}{l}\text { http://www.cgl.ucsf.edu/ } \\
\text { chimera/ }\end{array}$ \\
\hline
\end{tabular}

\subsubsection{Hardware}

Table 2.10: Used Hardware(local device)

\begin{tabular}{l|ll}
\hline Hardware & Type & \\
\hline Processor & Intel(R)Core(TM)i7-4470 CPU \\
& $@ 3.40 \mathrm{Ghz}$ \\
Memory & $16.0 \mathrm{~GB}$ \\
System type & 64 -bit \\
\hline
\end{tabular}




\section{Chapter 3}

\section{Results}

An experiment is a question which science poses to Nature, and a measurement is the recording of Nature's answer.

- Max Planck

\subsection{Cohesin}

The cohesin-related part of this work describes the generation of the first three-dimensional reconstruction of the human cohesin complex by single particle electron microscopy. 3D models of cohesin complexes with varying protein composition are obtained and compared to assign the containing protein subunits to specific densities. Accordingly, in the following sections, different cohesin complexes will be introduced. To distinguish them, the following nomenclature is introduced (Table 3.1). Corresponding final 3D models are defined by a tag (t1-t6) to allow easy attribution in written text:

\subsubsection{Visualisation of Full-length Cohesin}

Electron micrographs of human full-length cohesin prepared by low angle $\mathrm{Pt} / \mathrm{C}$ rotary shadowing provided deeper insights into the overall architecture of tetrameric cohesin, with its hinge region, a globular domain, the nucleotide binding domain (NBD), and two interconnecting arms, the coiled-coils (Anderson et al., 2002; Huis in 't Veld, 2013) (see Figure 3.1b). Open and closed conformations (depending on Scc1) were induced biochemically and thereby the DNA exit gate was characterised (Huis in 't Veld et al., 2014). In the closed state of this exit gate, the ring-like structure is apparent (see Figure $3.1 b)$.

Initial efforts focused on the 3D reconstruction of this full-length cohesin complex by single particle EM. A GraFix-treated full-length cohesin sample was examined by negative stain electron microscopy. Most complexes showed a "cudgel-like" appearance (proposed closed conformation in Figure 3.1d), while some complexes appeared similar to a "pair of 
Table 3.1: Cohesin nomenclature

\begin{tabular}{|c|c|c|c|}
\hline Name & Composition & Mol. mass & Related models (tag) \\
\hline Full-length cohesin & $\begin{array}{l}\text { Full-length Smc1 (140 kDa) } \\
\text { Full-length Smc3 (143 kDa) } \\
\text { Scc1 ( } 72 \mathrm{kDa}) \\
\text { SA1 (145 kDa) }\end{array}$ & $500 \mathrm{kDa}$ & only $2 \mathrm{D}$ model \\
\hline Bonsai cohesin tetramer & $\begin{array}{l}\text { Smc1 }^{\text {bonsai }}(72 \mathrm{kDa}) \\
\text { Smc3 }{ }^{\text {bonsai }}(75 \mathrm{kDa}) \\
\text { Scc1 }(72 \mathrm{kDa}) \\
\text { SA1 }(145 \mathrm{kDa})\end{array}$ & $364 \mathrm{kDa}$ & $\mathrm{t} 1, \mathrm{t} 3, \mathrm{t} 4$ \\
\hline Pds5 $\cdot$ bonsai cohesin tetramer & $\begin{array}{l}\text { Smc1 }^{\text {bonsai }}(72 \mathrm{kDa}) \\
\text { Smc3 } 3^{\text {bonsai }}(75 \mathrm{kDa}) \\
\text { Scc1 }(72 \mathrm{kDa}) \\
\text { SA1 }(145 \mathrm{kDa}) \\
\text { Pds5B }(165 \mathrm{kDa})\end{array}$ & $529 \mathrm{kDa}$ & $\mathrm{t} 2$ \\
\hline Pds5 $\cdot$ bonsai cohesin trimer & $\begin{array}{l}\text { Smc1 }^{\text {bonsai }}(72 \mathrm{kDa}) \\
\text { Smc3 }{ }^{\text {bonsai }}(75 \mathrm{kDa}) \\
\text { Scc1 }(72 \mathrm{kDa}) \\
\text { Pds5B (165 kDa) }\end{array}$ & $384 \mathrm{kDa}$ & t5 \\
\hline 2xRFP bonsai cohesin tetramer & $\begin{array}{l}\text { Smc1 }^{\text {bonsai }}(72 \mathrm{kDa}) \\
\text { Smc3 }{ }^{\text {bonsai }}(75 \mathrm{kDa}) \\
\text { Scc1-2xRFP }(125 \mathrm{kDa}) \\
\text { SA1 (145 kDa) }\end{array}$ & $417 \mathrm{kDa}$ & t6 \\
\hline Wapl $\cdot$ Pds5 $\cdot$ bonsai cohesin tetramer & $\begin{array}{l}\text { Smc1 }^{\text {bonsai }}(72 \mathrm{kDa}) \\
\text { Smc3 } 3^{\text {bonsai }}(75 \mathrm{kDa}) \\
\text { Scc1 }(72 \mathrm{kDa}) \\
\text { SA1 }(145 \mathrm{kDa}) \\
\text { Pds5B }(165 \mathrm{kDa}) \\
\text { Wapl }(150 \mathrm{kDa})\end{array}$ & $679 \mathrm{kDa}$ & no final 3D model \\
\hline
\end{tabular}

cherries", where the bulky ends were not connected (proposed open conformation in Figure 3.1d). Interestingly, these hypothetical open conformations are asymmetric, possibly due to bound SA1/Scc1 at one of the Smc NBDs. However, the nature of the particles in the apparently open conformation is not known. In contrast to low angle $\mathrm{Pt} / \mathrm{C}$ rotary shadowing results, full-length cohesin complexes with a ring-like appearance were not observed. The distinct appearance (ring vs. cudgel) may result from different sample preparation strategies for $\mathrm{PT} / \mathrm{C}$ rotary shadowing and negative staining or represent distinct functional states.

The sample appeared not inadequate for image processing regarding the obvious sample heterogeneity related to the $\sim 50 \mathrm{~nm}$ long flexible coiled-coils. We decided to generate a complex with reduced coiled-coil length, which was expected to represent a less dynamic complex and to be more appropriate for EM image processing. According to an example where the elongated coiled-coil containing Ndc80 complex was shortened and successfully crystallized (Ciferri et al., 2008), these complexes were termed "bonsai" cohesin. 

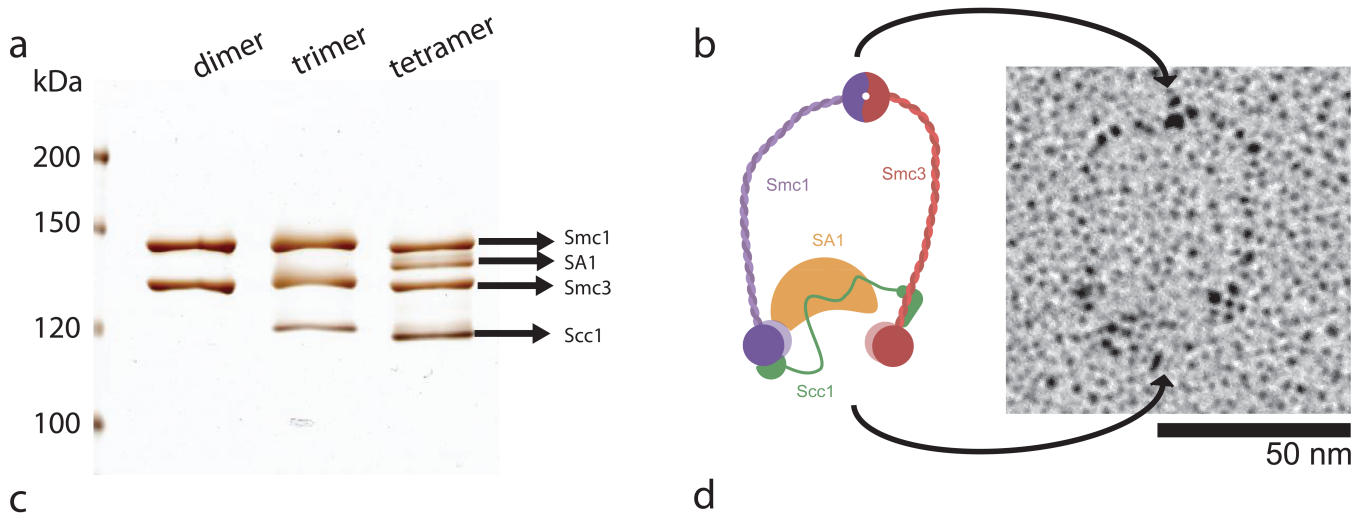

d
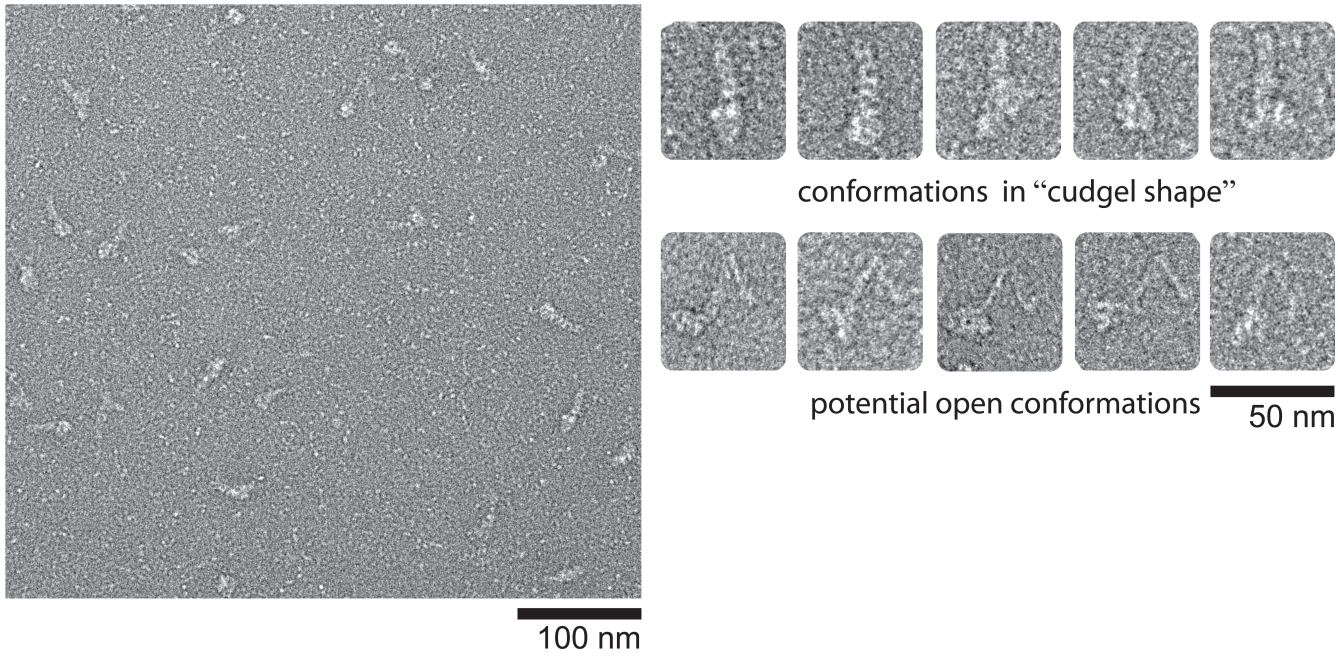

Figure 3.1: 2D Representation of Full-lengh Cohesin. a: Dimer (Smc1/3), Trimer (Smc1/3 + Scc1) and Tetramer (Smc1/3 + Scc1 + SA1) were expressed using the multibac system and purified as explained (see Section 2.1.1) (more details in Huis in 't Veld et al. (2014)). Shown is the comparison by SDS-PAGE, with all expected bands. b: The scheme of cohesin tetramer is shown in comparison to a low angle $\mathrm{Pt} / \mathrm{C}$ rotary shadowing image of cohesin in a closed ring-like conformation (Huis in 't Veld, 2013). The Smc1/3 hinge domain and the Smc1/3 NBDs are visible. Between the Smc1/3 NBDs a density is visible, which is expected to correspond to SA1 and parts of Scc1. Different from Figure 1.6, the Smc1/3 NBDs are not in an Smc1/3 head engaged state. c: An electron micrograph with negatively stained full-length cohesin (GraFix preparation) shows cohesin in different conformations. The "cudgel shape" may result from sample preparation, so that the coiled-coils are attached to each other (in distinction to b). d: Individual complexes were selected from several micrographs and were grouped. Two different forms are identified, an "cudgel shape"-like conformation and an potential open conformation. It is not known whether there is a functional implication of these two conformations or if they are a result of sample preparation. 


\subsubsection{Bonsai Cohesin Tetramer}

\subsubsection{Engineering of Bonsai Cohesin Tetramer}

Coiled-coil prediction algorithm and genetic engineering allowed Dr. Pim Huis in't Veld to remove most of the predicted coiled-coil region while a small flexible segment to connect the globular NBDs and the hinge region was retained. After a two-step purification, the complex was shown to be stoichiometric and pure (Figure 3.2d), and the complex' integrity was validated by $\mathrm{Pt} / \mathrm{C}$ rotary shadowing and negative staining (Figure 3.2b and c). Moreover, ProteoPlex assays (Figure 3.3c), ATPase activity assays (Figure A.2), chemical cross-linking followed by mass spectrometry (Figure 4.3) and size exclusion chromatography validated the complex integrity (Huis in 't Veld, 2013). Adequate amounts of bonsai cohesin tetramer for single particle EM purposes were generated. Bonsai cohesin tetramer is an active ATPase with reaction rates comparable to full length cohesin (Figure A.2). Further details related to the purification and the genetic engineering strategy are described elsewhere (Huis in 't Veld, 2013).

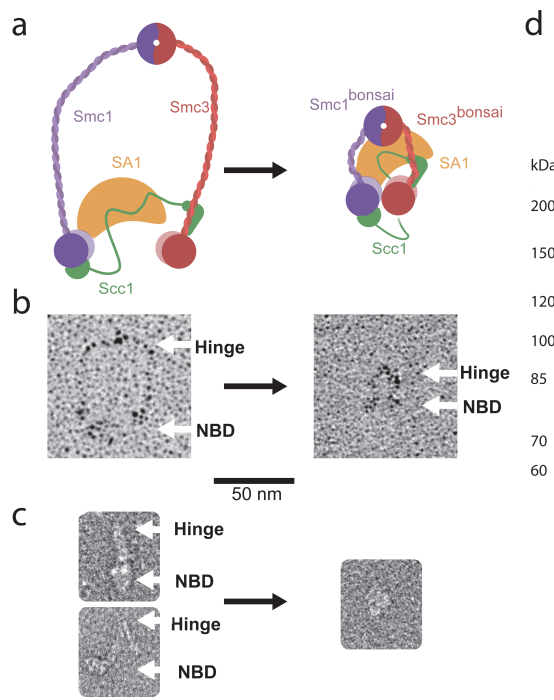

d

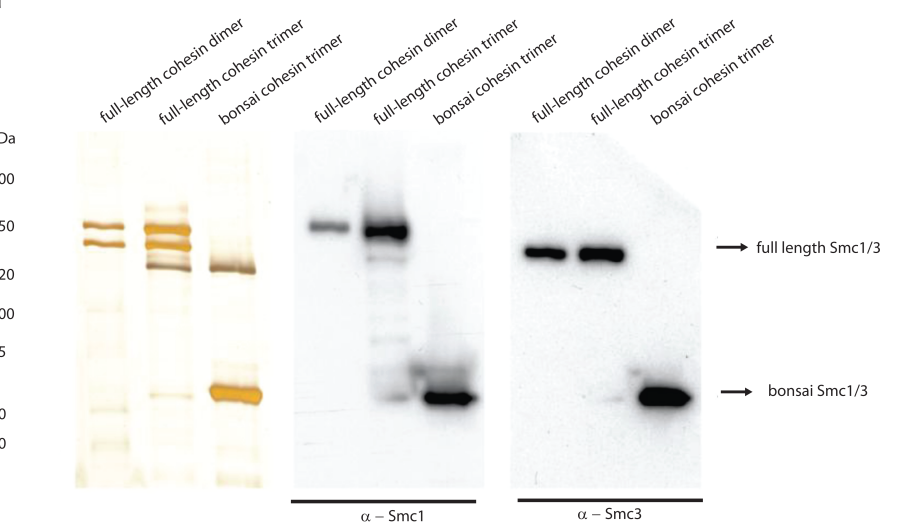

Figure 3.2: Engineering of Bonsai Cohesin Tetramer. a: Schematically, the removal of most of the coiled-coil segment is shown from full-length cohesin (left), generating the bonsai cohesin tetramer (right). b: Full-length cohesin (left) and bonsai cohesin tetramer (right) is compared in electron micrographs of samples prepared by low angle Pt/C rotary shadowing. c: Two full-length cohesin conformations (left) are compared with the bonsai cohesin tetramer (right) prepared in negative stain. d: In SDS-PAGE stoichiometric and pure bonsai cohesin trimer $\left(\mathrm{Smc1} / 3^{\text {bonsai }}+\mathrm{Scc} 1\right)$ was validated in comparison to full-length cohesin dimer $(\mathrm{Smc1} / 3)$ and trimer $(\mathrm{Smc1} / 3+\mathrm{Scc} 1)$. Immunoblotting with $\alpha$-Smc1/3-specific antibodies showed that truncated versions of both Smc1 and Smc3 are present in the bonsai cohesin complex. 


\subsubsection{Optimised Sample Preparation}

The requirements for sample homogeneity are high in single particle EM to yield highresolution structures. Therefore, the GraFix protocol and the identification of stabilising conditions for complexes with high throughput screens (ProteoPlex) are constantly optimised in the lab of Prof. Dr. Holger Stark (Haselbach, 2014). In this context, Pmaleimidophenyl isocyanate (PMPI) was shown to stabilise ribonucleoproteine (RNP) complexes (personal communication with Dr. Ashwin Chari) and was tested with further molecular complexes. GraFix with reverse GA gradient (increasing from top to bottom) and PMPI gradient (decreasing from top to bottom) was used for bonsai cohesin tetramers and yielded very homogeneous and monodisperse particles in negative stain micrographs (Figure 3.3b). In an equivalent non-cross-linked gradient purification, the fractions were analysed by SDS-PAGE (Figure 3.3a). The formation of a stable complex containing all subunits was shown (Figures 3.3a and 3.4f). It was assured by silver stain dot blots that the peak fraction in GraFix correlates with the peak fraction identified in SDS-PAGE. Moreover, the stability of the tetrameric bonsai cohesin was increased by the addition of ATP $\gamma \mathrm{S}$ as defined in a ProteoPlex screen. Different buffer conditions were tested and HEPES pH 7.5 with ATP $\gamma \mathrm{S}$ was identified as a favourable condition (Figure 3.3c). In this work no quantitative strategy to further optimise the sample preparation was performed, as negative stain micrographs showed satisfying conditions for image processing. Details can be reviewed in Huis in 't Veld (2013).

\subsubsection{Initial Structural Insights}

A dataset of $\sim 52,000$ negatively stained particle images was recorded (Figure 3.4a). After initial data pre-processing and CTF correction, 33,500 particle images (64\%) remained. Using this dataset the first complete 3D model of bonsai cohesin tetramer was generated (3D model t1 in Figure 3.4b). By Fourier shell correlation (FSC), the 3D model was determined at a resolution of $\sim 30 \AA$ ( 0.5 criterion) (Figure 3.4c). Auto-segmentation of the 3D model yielded four segments (Figure $3.4 \mathrm{~d}$ ). A finer segmentation seemed inappropriate regarding the low resolution of the 3D model. Two segments (purple and red) are similar in form and size and are opposing each other. They are connected by a bigger segment at the top (head segment: brown). At the bottom, the two opposing segments almost touch each other (purple and red). On the backside, the biggest segment forms a clasp (orange) around the area that connects the opposing segments, thereby connecting all three other segments. The enclosed volume of each segment was calculated after the density threshold was chosen to fit the expected molecular mass. Interestingly, the two opposing segments (bottom left, bottom right) had a similar enclosed volume corresponding to $72 \mathrm{kDa}$ and $78 \mathrm{kDa}$, respectively. The head segment corresponds to $90 \mathrm{kDa}$, and the backside segment 


\section{a}

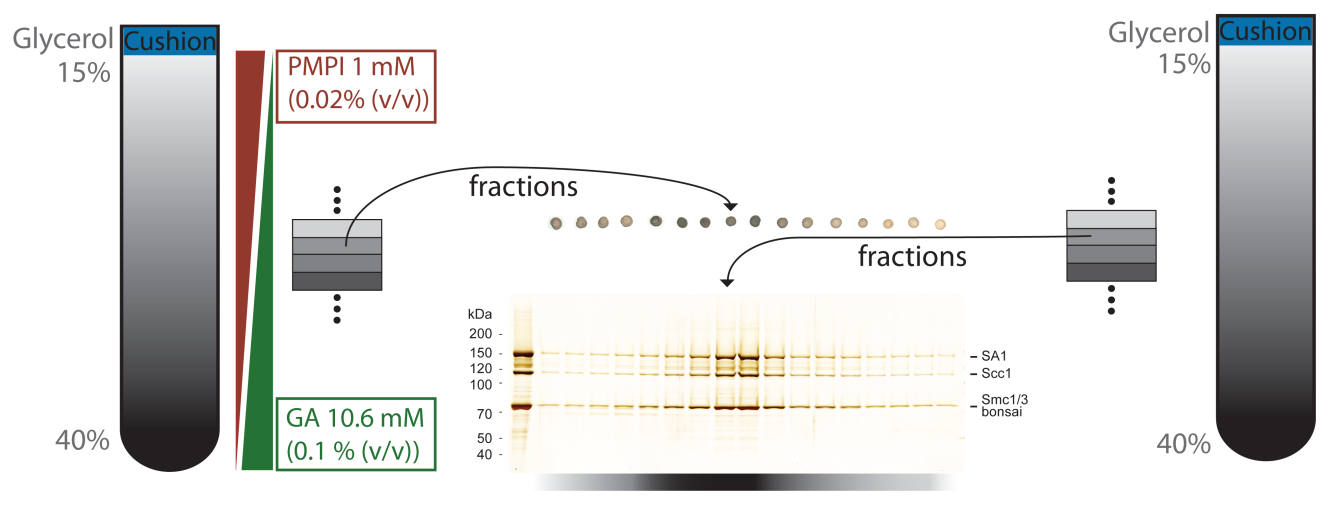

b

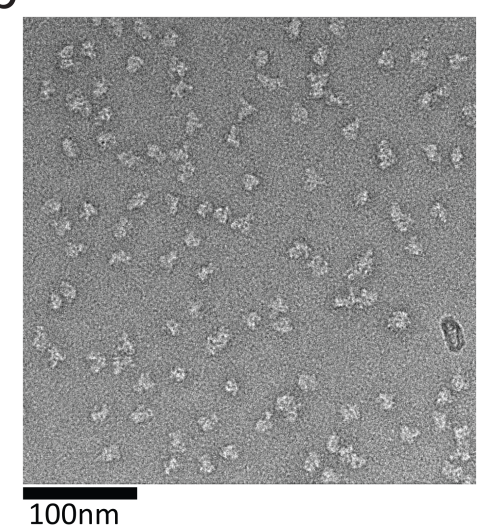

C

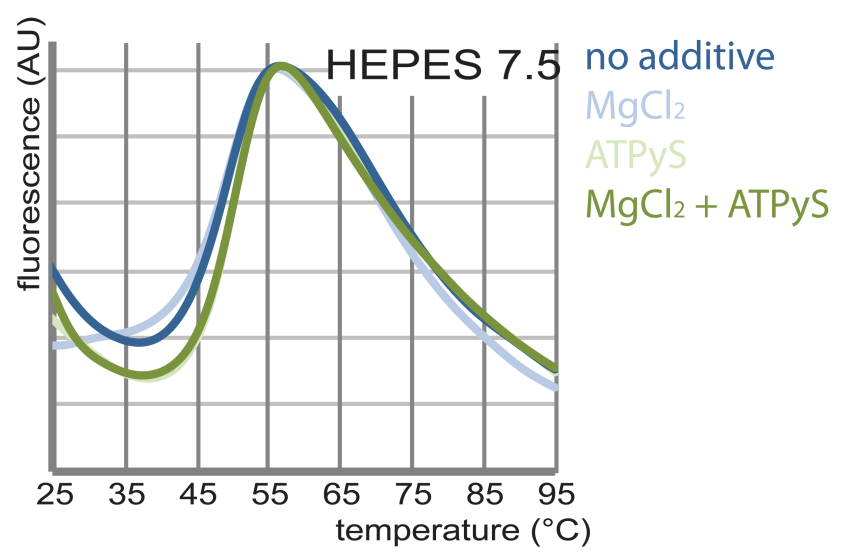

Figure 3.3: Optimised Sample Preparation. a: A modified GraFix protocol is used for the stabilisation of bonsai cohesin tetramer. As illustrated, GA and PMPI are added in a reverse gradient (left). Two equivalent gradients are prepared, while only in one case cross-linkers are added. The gradients are fractionated equivalently and the GraFix gradient fractions are used for batch silver staining (dot blot) and negative stain grid preparation. The cross-linker-free gradient fractions (right) are used for SDS-PAGE analysis. By comparison of both, fractionated gradients in SDS-PAGE and EM micrographs, peak fractions containing homogenous monodisperse and complete complexes were identified. $\mathbf{b}$ : An exemplary electron micrograph of an initial screening of bonsai cohesin tetramer is shown. Most particles are homogeneous and monodisperse. c: The ProteoPlex assay is used to define optimal buffer conditions for bonsai cohesin tetramer. Different buffer systems were tested. Here, only HEPES pH 7.5 is shown. ATP $\gamma \mathrm{S}$ was shown to stabilise the complex. For all bonsai cohesin complexes used in this work, $183 \mu \mathrm{M}$ $\operatorname{ATP} \gamma\left(\mathrm{HEPES} \mathrm{pH} 7.5,2 \mathrm{mM} \mathrm{MgCl}_{2}\right.$ ) was added. 
(clasp) corresponds to $128 \mathrm{kDa}$ (in total $368 \mathrm{kDa}$ ). Regarding the limited reliability of the segmentation and the mass-calculations of low resolution structures, these were preliminary results giving insights into the rough topology of bonsai cohesin tetramer. The two opposing segments were assumed to represent the globular NBDs of the Smc1 ${ }^{\text {bonsai }}$ and Smc $3^{\text {bonsai }}$, respectively, while the head segment was proposed to correspond largely to the hinge domain. The biggest segment on the backside was proposed to match largely the SA1 subunit of $145 \mathrm{kDa}$. Scc1, which is known to span a huge distance within cohesin, is assumed to contribute to different areas of the 3D model. A more detailed and reliable assignment was only possible by the integration of data from different techniques (see Section 4.1.3 for final assignments). The angular distribution plot shows that the used images represent mainly polar regions, while equatorial regions are underrepresented.

\subsubsection{Pds5 - Bonsai Cohesin Tetramer}

Pds5 plays a major role in cohesin regulation, though many aspects of its regulation are not understood. As it recruits Wapl and Sororin, Pds5 fulfils a double role. Wapl promotes cohesin release from chromosomes, whereas Sororin stabilises sister chromatid cohesion (Nishiyama et al., 2010). The open question concerning the function, the unknown binding site to cohesin and the lacking structural characterisation indicate Pds5 as a very interesting target for single-particle EM studies in an cohesin-bound state. Moreover, Pds5-bound cohesin (529 kDa) may break the pseudo-symmetric nature of bonsai cohesin tetramer $(364 \mathrm{kDa})$. Stronger asymmetry and increased size may allow an improved 3D model of bonsai cohesin. In this work, the term "Pds5" will be used to refer to the isoform "Pds5B".

\subsubsection{Biochemical Integrity of Pds5 Binding to Cohesin}

It was shown that Pds5 binds full-length cohesin and bonsai cohesin (Figure 3.5a) in a Scc1-dependent manner (Huis in 't Veld, 2013). Pds5 was purified using a metal-chelate affinity purification and added in large excess to bonsai cohesin complexes that were immobilized on FLAG beads (2.1.1). The beads were subsequently washed to remove unbound Pds5, and Pds5 - bonsai cohesin tetramer was eluted by addition of FLAG peptide. The sample was used for single particle EM after GraFix stabilisation. GraFix gradients were prepared equivalently to the bonsai cohesin tetramer sample. The presence of each subunit in the fractions was confirmed using SDS-PAGE from a non-fixated gradient. The peak fraction of the non-fixated gradient purification and the GraFix purification were shown to correspond to each other by SDS-PAGE and silver stain dot blot, respectively. By means of SDS-PAGE, the fraction used for data processing was 


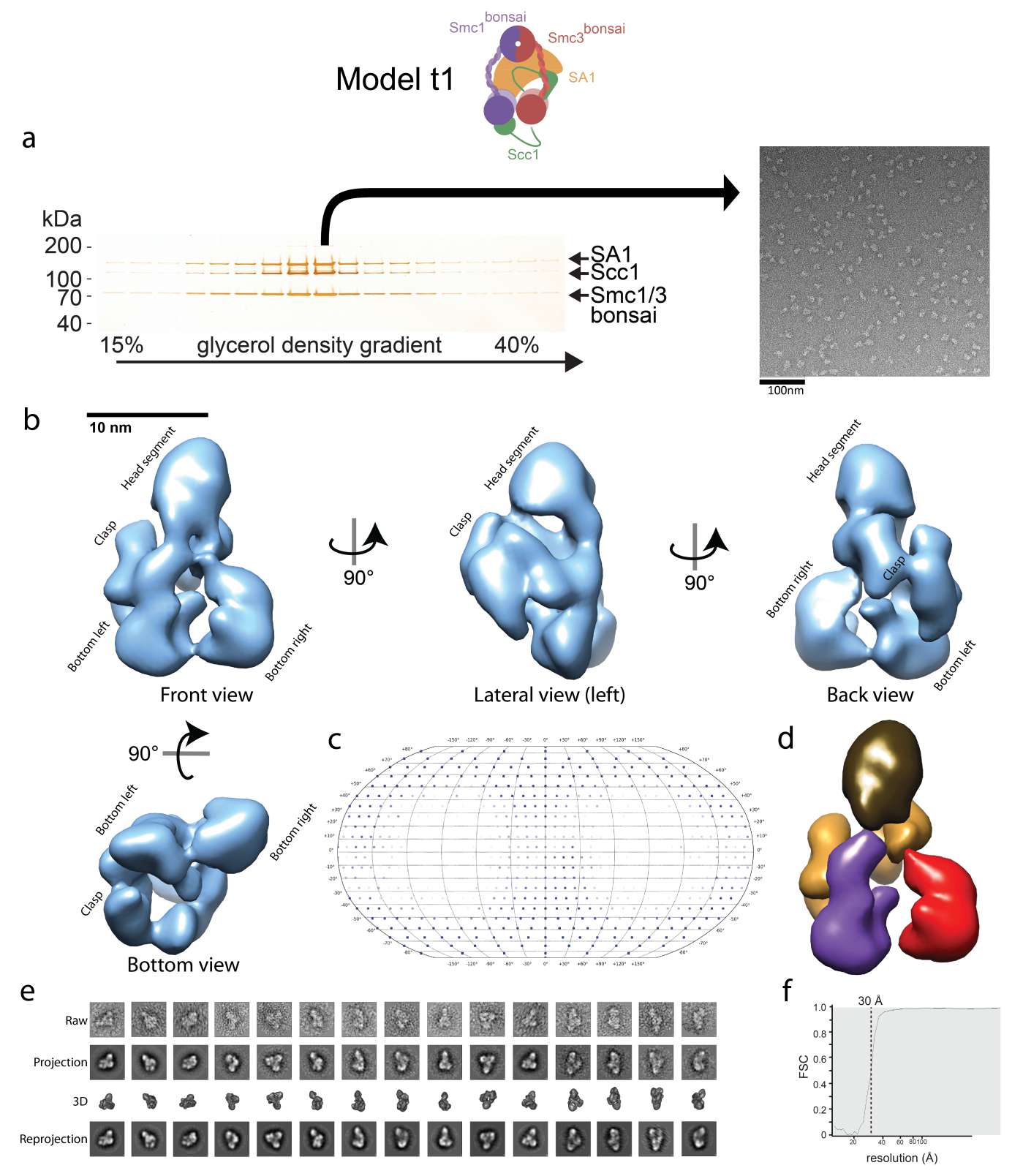

Figure 3.4: Initial Structural Insights into Bonsai Cohesin Tetramer (t1). a: In SDS-PAGE the peak fraction is shown in comparison to an exemplary electron micrograph of this dataset. A satisfying particle homogeneity is given. 33,500 particle images were used for the obtained 3D model. b: Front view, lateral view (left), back view and top view are depicted for the final obtained 3D model t1 of bonsai cohesin tetramer. Four different regions, according to the segmentation in c, are labelled within 3D model t1: The bottom left, the bottom right, the clasp and the head segment. c: In the euler plot, the angular distribution of projection images which were used to reconstruct the 3D model are shown. The euler angle $\beta$ and the euler angle $\gamma$ corresponds to the $\mathrm{y}$ axis and $\mathrm{x}$ axis, respectively. The low coverage in the equatorial regions may explain the elongated appearance of 3D model. d: The auto-segmentation using a watershed and scale-space filtering algorithm within Chimera (Pintilie et al., 2010) resulted in the following segmentation: head segment (brown), bottom left (purple), bottom right (red), clasp (orange). e: Comparison between raw images, projections, related 3D model perspectives and reprojections. f: By FSC (0.5 criterion), the 3D model was determined at a resolution of $\sim 30 \AA$. 
optically evaluated to contain stoichiometric amounts of each subunit. Moreover, a peak shift to heavier gradient fractions indicated Pds5 binding (Figure 3.5c). An excess of free Pds5 was identified in lighter fractions. In case of the first sample containing Pds5, potentially Wapl was present. However, by using a Wapl-specific antibody the complex was shown to contain only stoichiometric amounts of Wapl (Figure A.3). Moreover, a second sample was purified were potential Wapl contamination was precluded. Obtained 3D models of the first and the second dataset were shown to be equivalent. In summary, the binding of Pds5 was ensured. The complex was visually examined in negative stain micrographs and appeared homogeneous in size and monodisperse, while almost no aggregation was identified (Figure 3.5d).

\subsubsection{Structural Insights into Pds5 Binding}

48,411 particle images were selected and the dataset was reduced to 37,749 particle images (80\%) in an initial cleaning step. After CTF correction, 28,000 (58\%) particle images remained. A reference-free ab initio reconstruction of the dataset was performed. Accordingly, any emerging similarity between a 3D model of this dataset and the 3D model t1 of bonsai cohesin tetramer is likely to be authentic. In addition, an arising additional density is likely to correspond to Pds5. This strategy has the advantage that the possibility of reference-based model bias is excluded. In case of a reference-based strategy, any similarity may be superimposed by the reference model.

Initially, the dataset was processed on 2D level to generate 500 class averages. These class averages were used in SIMPLE Prime to generate a start-up 3D model. Further processing (including resampling, 3D classification and refinement), yielded an improved 3D model. The overall shape of this 3D model is similar to the $3 \mathrm{D}$ model t1 of bonsai cohesin tetramer. Moreover, an additional density was recognised, which was proposed to correspond to Pds5. Accordingly, this additional density was removed by a Gaussian mask to yield a $\Delta 3 \mathrm{D}$ model. The density recovery, in a refinement step with the $\Delta 3 \mathrm{D}$ model, proved this density to be authentic (see Section: Internal Validation 3.1.7.1: figure $3.12)$.

Interestingly, a supervised classification using both 3D models (original and $\Delta 3 \mathrm{D}$ model) showed that only $36.4 \%$ (5,644 images) of the images were assigned to the original 3D model. $63.6 \%$ (9,868 images) of the images were assigned to the $\Delta 3 \mathrm{D}$ model where the additional density was removed (Figure $3.7 \mathrm{a}$ ). A second dataset was recorded as the necessity of sorting and the resulting small number of particle images was expected to be limiting for further image processing. The new dataset contained $\sim 50,000$ particle images after pre-processing (cleaning and CTF correction). The new dataset was divided with respect to the expected Pds5 density by supervised classification. Only 37.5\% (18,747 images) of 
a

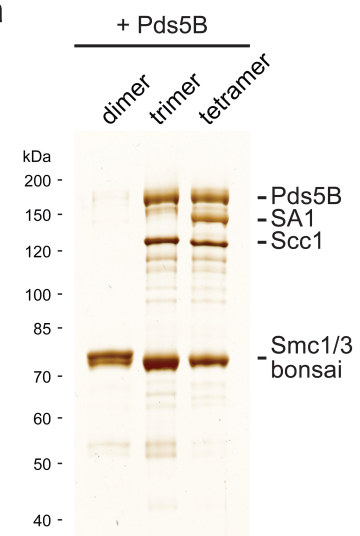

C

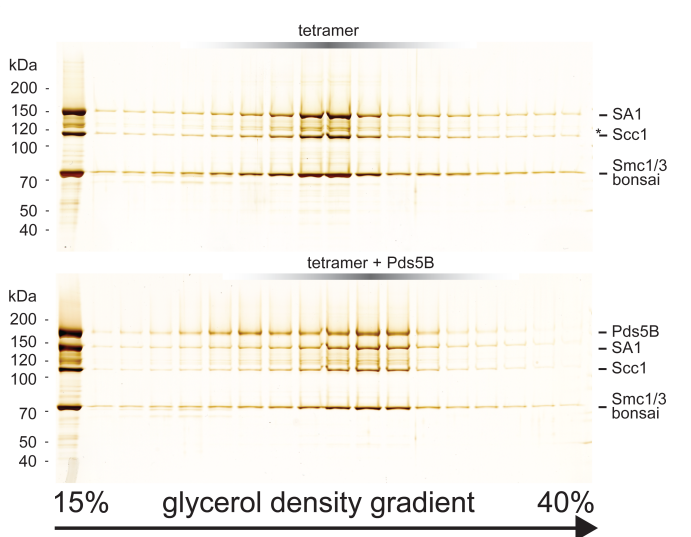

b
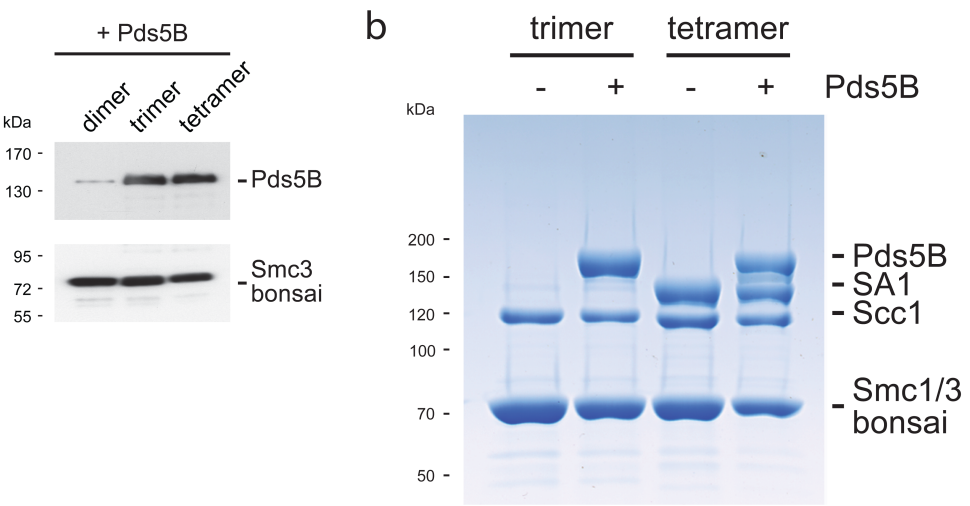

d

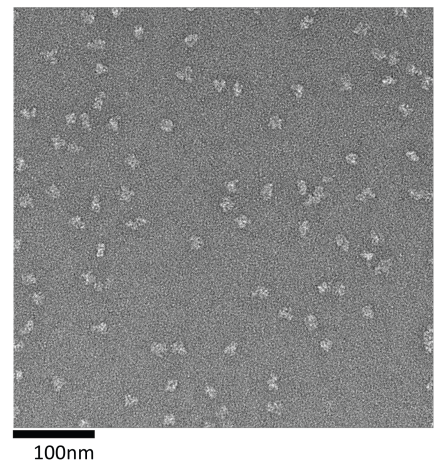

Figure 3.5: Biochemical Integrity of Pds5 Binding to Cohesin. a: A sample containing Pds5 was added to bead-immobilised Smc1/3 ${ }^{\text {bonsai }}$ dimer, trimer (+ Scc1) and tetramer (+ SA1) during protein purification. The eluate was shown to contain bound Pds5 in the case of the trimer and the tetramer but not in the case of the dimer ( $\alpha$-Pds5-specific antibody). Accordingly, Pds5 binding was shown to be Scc1-dependant. The use of Smc1/3bonsai did not seem to reduce Pds5 binding. The first Pds5 - bonsai cohesin tetramer dataset was recorded from this sample (28,000 images after CTF correction). A second dataset was recorded from a sample, where potential Wapl contamination of the first sample were prevented $(\sim 50,000$ particle images after CTF correction). b: Pds 5 was bound in stoichiometric amounts to complexes with or without SA1. Accordingly, four differnt complexes were available. A strong Pds5 band is visible in the case of the Pds5-added sample. c: GraFix was performed on the Pds5-bound and Pds5-free bonsai cohesin sample as described in Figure 3.3. A cross-linker-free gradient was fractionated and analysed in SDS-PAGE. Pds5 binding induces a shift of the peak fraction to deeper fractions compared to the Pds5-free tetramer, confirming the formation of Pds5 - Bonsai Cohesin Tetramer. d: The visual appearance of electron micrographs attest homogeneous and monodisperse particles in decent quality for image processing. 
the dataset were assigned to the 3D model with the additional density. Thus, independent of the used dataset, a constant ratio of particles with and without this density was found. Based on these results, a compositional heterogeneity regarding the Pds5 binding is likely. Possibly, Pds5 is not as stable bound as other subunits, allowing the formation of Pds5-free complexes, despite the excess of added Pds5 during purification.

Using the Pds5-bound fraction of the dataset (37.5\%; 18,747 images), a series of 3D classifications steps resulted in a more detailed 3D model of Pds5 - bonsai cohesin tetramer (3D model t2) (Figure 3.6). In comparison to the 3D model of the bonsai cohesin tetramer, a clear additional density can be defined, while the overall appearance of the obtained 3D model is very similar. The structure was determined at a resolution of $35 \AA$ ( 0.5 criterion) (Figure 3.6f). The 3D model has a less elongated appearance than 3D model t1. The angular distribution map confirmed a more homogeneous distribution of projection images. Different from the 3D model t1, all projections were equally represented (Figure 3.6b). Accordingly, the 3D model is expected to be more isotropic. The performed segmentation emphasises the similarity to the 3D model t1. Two similar globular segments at the bottom (bottom left in purple, bottom right in red) are connected to a segment at the top with a central cavity (head segment: brown). The connection between the head segment and the bottom left as well as the bottom right segment is approximately in the center of the front view. On the backside, a clasp forms around the middle of the 3D model, similar as in 3D model t1. Additionally, and in contrast to 3D model t1, between the globular segments (bottom left, bottom right) at the bottom, a long density spans from the clasp down to the bottom and up to almost the center of the front view. This density is expected to correspond to Pds5. The volume of the extra density matched approximately the expected mass of Pds5 with $165 \mathrm{kDa}$. A comparison of the isolated Pds5 density with a homology model of Pds5 is given in Figure A.4. A cavity appeared centrally in the head segment, which approximately matches the size of the cavity within the crystal structure of the hinge domain (see Figure 3.7e). The two interconnecting arms are of similar length, and they are proposed to correspond to the coiled-coil fragments connecting the globular Smc1 $/ 3^{\text {bonsai }}$ NBDs with the hinge domain. The clasp on the backside contains a small and a big density.

\subsubsection{Compositional and Conformational Heterogeneity}

Only by splitting the dataset regarding the occupancy of the proposed Pds5 density, it was possible to obtain the improved $3 \mathrm{D}$ model t2. Thus, the dataset is likely to contain a mixture of Pds5-bound and Pds5-free bonsai cohesin tetramer. If this hypothesis is correct, one should obtain a 3D model similar to t1 (bonsai cohesin tetramer) from the subset (62.5\%; 31,309 images) assigned to the $\Delta$ 3D model. 


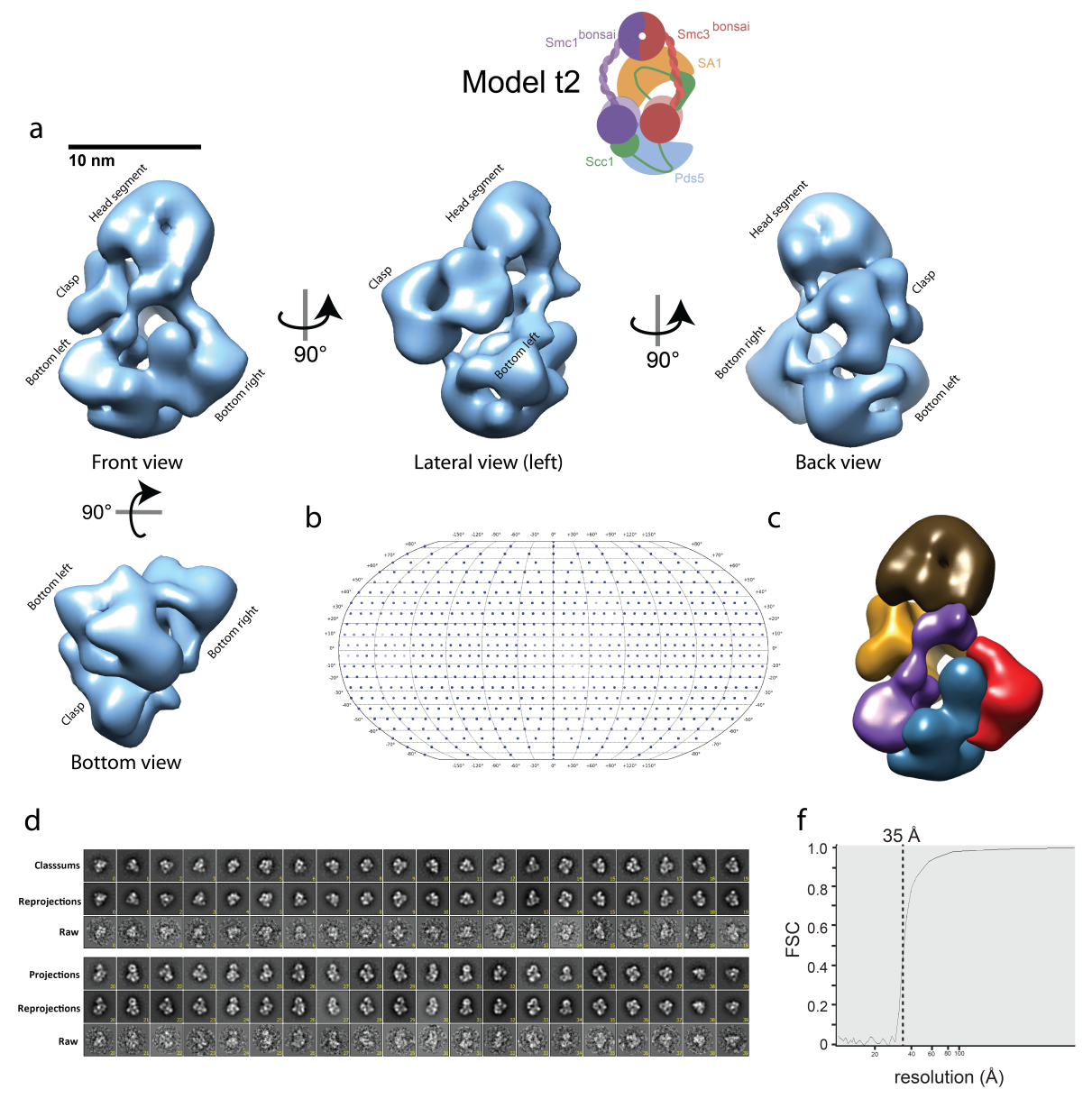

Figure 3.6: First Structural Insights into Pds5 - Bonsai Cohesin Tetramer (t2). a: The 3D model of Pds5 - bonsai cohesin tetramer is shown. The additional density which is proposed to correspond to Pds5 can be seen at the bottom between the bottom left segment and the bottom right segment. It reaches up to almost the middle of the front view. In the back view, the expected Pds5 density connects to the clasp. The probable Pds5 density has a strong curvature, bending approximately $90^{\circ}$ (left lateral view). This is in accordance to the homology model of Pds5. A direct comparison between the isolated proposed Pds5 density and the Pds5 homology model is depicted in Figure A.4. b: The euler plot shows a homogeneous distribution of projection images. Moreover, the 3D model does not appear as elongated as $3 \mathrm{D}$ model $\mathrm{t} 1$, where a underrepresented projections were identified in the euler plot. The euler angle $\beta$ and the euler angle $\gamma$ correspond to the $\mathrm{y}$ axis and $\mathrm{x}$ axis, respectively. c: A manual segmentation was performed according to the comparison between the 3D models t1-t4. A very similar segmentation is possible (Head segment (brown), bottom left (purple), bottom right (red), clasp (orange)) for the 3D models t1-t4, while only 3D model t2 shows the proposed Pds5 density (blue). d: Selected projections, reprojections and related raw images of the 3D model t2 are depicted. e: By FSC ( 0.5 criterion), the 3D structure was determined at a resolution of $35 \AA$. 

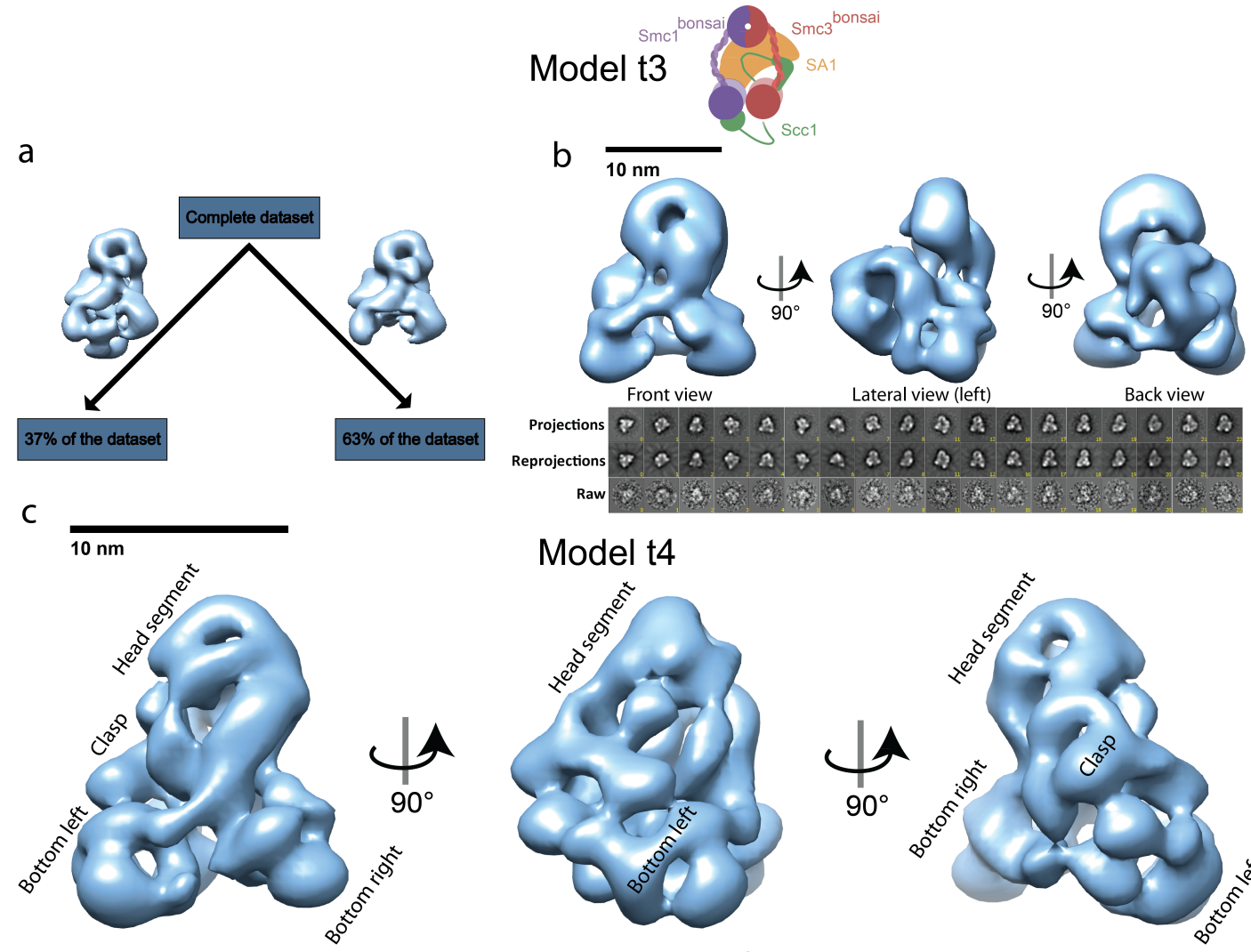

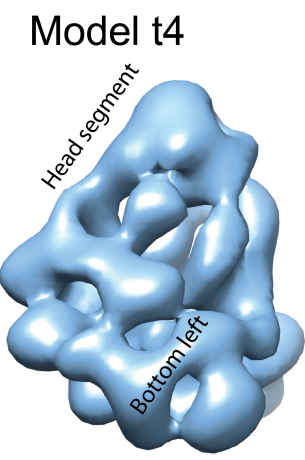

Lateral view (left)

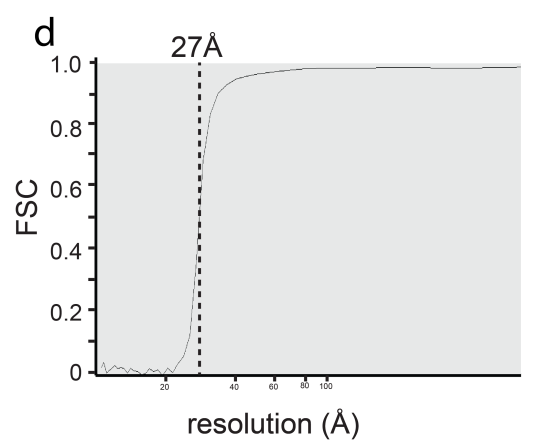

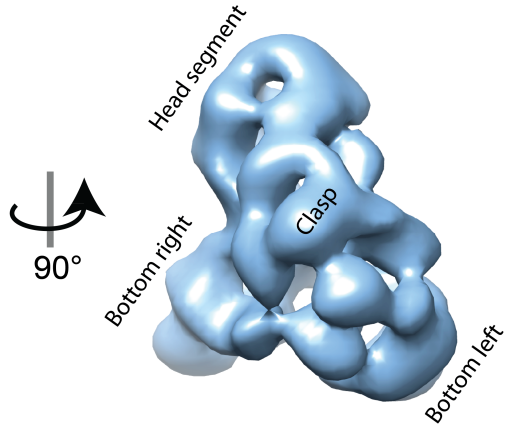

Back view

e

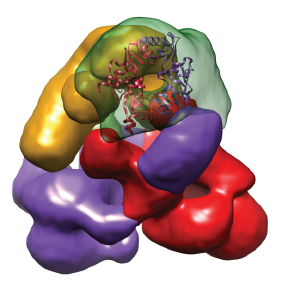

Bottom view

Figure 3.7: Sorting for Pds5 Binding Enables an Improved 3D Model of Bonsai Cohesin Tetramer (t3 \& t4). a: Pds5 was identified between the bottom left and bottom right segment. By sorting with this 3D model and a $\Delta 3 \mathrm{D}$ model (removed Pds5 density), the dataset of Pds5 - bonsai cohesin tetramer has been divided into two distinct populations. The left population contained an additional density and was refined to yield 3D model t2 (see Figure 3.6) and the right population was refined into 3D models $\mathrm{t} 3$ and t4. Only $37 \%$ of the dataset were sorted for the Pds5-bound fraction. b: $3 \mathrm{D}$ model t3 appears very similar to $3 \mathrm{D}$ model t1. An equivalent segmentation as in the case of $3 \mathrm{D}$ model t1 into four segments was possible. Exemplary projections, reprojections and related raw images are shown. c: $3 \mathrm{D}$ model $\mathrm{t} 4$ was refined by using $3 \mathrm{D}$ model $\mathrm{t} 3$ as reference. The $3 \mathrm{D}$ model $\mathrm{t} 4$ appears very similar but more detailed. Noteworthy are the more defined connections between the bottom left segment, bottom right segment and the head segment. They are likely to correspond to the shortened coiled-coils. The visible X shape may correspond to a cross-over of these coiled-coils. The head segment and the donut-shaped opening of the head segment appear smaller. d: The resolution was determined by FSC (0.5 criterion) as $\sim 27 \AA$. Accordingly, this structures is the best resolved structure of bonsai cohesin. e: The hinge domain (PDB: 2WD5) is overlayed with the head segment to show the equivalent size of the cavity. (red: Smc1 ${ }^{\text {bonsai }}$, blue: $\mathrm{Smc} 3^{\text {bonsai }}$ ). 
Accordingly, this hypothesis was tested. By 3D classification of this subset, a 3D model t3 was obtained, revealing a high degree of structural similarity with $3 \mathrm{D}$ model t 1 of bonsai cohesin tetramer. Using the $\Delta 3 \mathrm{D}$ model as reference, the supposed Pds5 density was not recovered, validating that this fraction contains Pds5-free bonsai cohesin tetramer. Further 3D-classification resulted in an improved 3D model t4. The 3D model t4 was determined at a resolution of $\sim 27 \AA$ ( 0.5 criterion). This $3 \mathrm{D}$ model is the best resolved model of bonsai cohesin. Both 3D models ( $\mathrm{t} 3$ and $\mathrm{t} 4$ ) appear less elongated than the initial $3 \mathrm{D}$ model $\mathrm{t} 1$, which is in accordance to 3D model t2 of Pds5 - bonsai cohesin tetramer. Conclusively, the elongation of $3 \mathrm{D}$ model $\mathrm{t} 1$ seems to be an artefact due to missing views. In 3D model t4, the proposed hinge domain becomes more defined and the bottom left and bottom right segments (the proposed Smc1/3 $3^{\text {bonsai }}$ NBD) are more detailed. Even more, the interconnecting arms are more defined as their connection to the proposed hinge domain (head segment) is visible. In this 3D model, the interconnecting arms appear to be crossed, as the left bottom density seems to be connected to the right part of the head segment and the right bottom density seems to be connected to the left part of the head segment.

Besides the compositional heterogeneity regarding the Pds5 occupancy, there is a large amount of conformational heterogeneity, which may be responsible for the limited resolution. In a 3D classification approach, several different 3D models with slightly varying positions of the described segments have been defined (Figure 3.8a). This may relate to the coiled-coil fragments and flexibility of HEAT repeat proteins.

These previously described results were obtained from negatively stained samples. However, high resolution information can only be accessed with datasets of vitrified sample. Cryo datasets of bonsai cohesin tetramer and Pds5 - bonsai cohesin tetramer were generated (Figure 3.8b). A dataset of Pds5 - bonsai cohesin tetramer with 100,000 images was pre-processed, and reference-free 2D image processing resulted in initial class averages (Figure 3.8c). On 2D level, cryo class averages appear similar to initial class averages generated from negative stain data. However, it was not possible to increase the quality of the data or generate reasonable 3D models by angular reconstitution. Regarding the size, the low contrast of vitrified sample and the heterogeneity of the sample, it appears difficult to generate a high resolution model ab initio. Beside the reference-free approach, 3D model t2 (negative stain data) was used as reference for this dataset (Figure 3.8c). However, 3D classification did not result in a credible 3D model. Further sorting and processing may allow to obtain an improved 3D model. The compositional and conformational heterogeneity may be related to the difficulties for low-contrast datasets of vitrified sample.

In summary, the first reference-free 3D model of Pds5 - bonsai cohesin tetramer was defined. The Pds5 density has been allocated within this 3D model t2. Moreover, an 


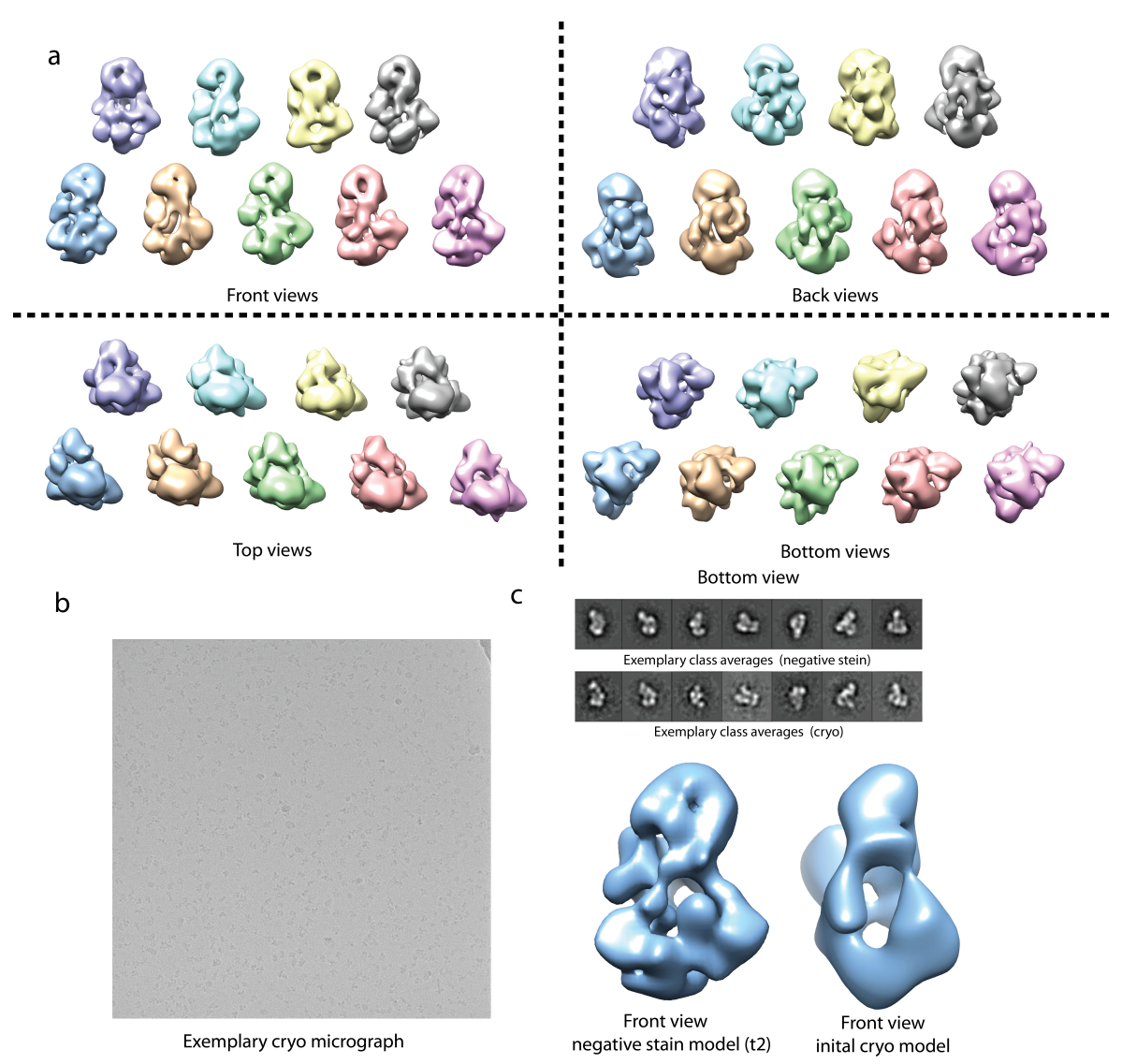

Figure 3.8: Heterogeneity within Pds5 - Bonsai Cohesin Tetramer. a: Front views, back views, top views and bottom views of nine obtained 3D models of Pds5 · bonsai cohesin tetramer are shown. The light purple 3D model corresponds to the 3D model t2. Differences are highlighted by grey boxes and are proposed to correspond to the conformational heterogeneity of bonsai cohesin. This heterogeneity may be related to the flexibility of the remaining coiled-coil fragments. In the front view, the most prominent heterogeneity affects the proposed coiled-coils. In the dark purple 3D model, they almost disappear. In the back view, the heterogeneity of the clasp is depicted. While the overall shape is similar, the connections within the claps and with other areas change. For example, the connection of the clasp to the proposed Pds5 density varies. The top view shows the heterogeneity within the head segment. Comparing the green and the yellow 3D model, a different in-plane rotation can be seen. The bottom view shows the heterogeneity within the proposed Pds5 density. Most prominent is the difference in the yellow 3D model. There, the presumable Pds5 density is very weakly defined. b: An electron micrograph of vitrified Pds5 - bonsai cohesin tetramer is depicted. Obviously, the individual particles are harder to define than in the negatively stained sample, as the contrast is lower. c: At the top, a comparison between selected class averages of the negatively stained sample and similar class averages of the vitrified sample is shown. At the bottom, the 3D model t2 obtained from negatively stained sample is compared to a 3D model of the vitrified sample. Further processing may allow to generate improved 3D models from vitrified sample. 
improved 3D model of bonsai cohesin tetramer was generated. The Pds5 occupancy is around $40 \%$.

\subsubsection{Pds5 • Bonsai Cohesin Trimer}

By comparison of the obtained 3D models (bonsai cohesin tetramer and the Pds5 - bonsai cohesin tetramer) Pds5 was identified (Figure 4.1). The positions of further subunits were only assumed based on related to X-ray models (Smc1/3 $3^{\text {bonsai }}$ NBDs and the Smc $1 / 3^{\text {bonsai }}$ hinge domain; see Section 4.1.3). Especially, we aimed to localise SA1. According to Pt/C rotary shadowing results, SA1 is located near the Smc1/3 $3^{\text {bonsai }}$ NBDs (Figure 3.1b). In the crystal structure of SA2 (an isoform of SA1), a fragment of Scc1 is bound to the SA2 repeats (Figure 1.6). Our crosslinking data confirmed this interaction (Section 4.1.4; Figure 4.3). Thus, we aimed to define the position of SA1 by single particle EM. This is possible by omitting the SA1 subunit within the complex and comparing the output 3D model to previously described 3D models. Initial efforts with a bonsai cohesin trimer (without SA1 and Pds5) were not successful, possibly due to the very small size of the complex $(219 \mathrm{kDa})$. In the following section, the results of a Pds5 - bonsai cohesin trimer sample will be presented.

\subsubsection{Biochemical Integrity}

The protein purification of Pds5 - bonsai cohesin trimer is similar to the purification of Pds5 - bonsai cohesin tetramer, while only the addition of SA1 is omitted. Figure 3.5a/b shows that Pds5 can be stably bound to bonsai cohesin trimer. A pure sample has been generated as shown by a non-cross-linked gradient purification and subsequent SDS-PAGE analysis (Figure 3.9a). GraFix stabilisation was performed similarly as shown for the bonsai cohesin tetramer sample. The sample was prepared in negative stain. The homogeneity of the sample, as shown in initial micrographs, fulfils all prerequisites for single particle EM. In the defined peak fraction, the cross-linked Pds 5 - bonsai cohesin trimer showed a satisfying sample quality regarding homogeneity and concentration (Figure $3.9 \mathrm{~b})$.

\subsubsection{Structural Insights into SA1 Binding}

The recorded dataset contained 82,472 particle images, whereas only 41\% (34,074 images) remained after data pre-processing (cleaning and CTF correction). The stronger reduction in size compared to previous datasets may be due to automated particle selection, as more empty images were selected than in a semi-automated approach. For image processing, a 

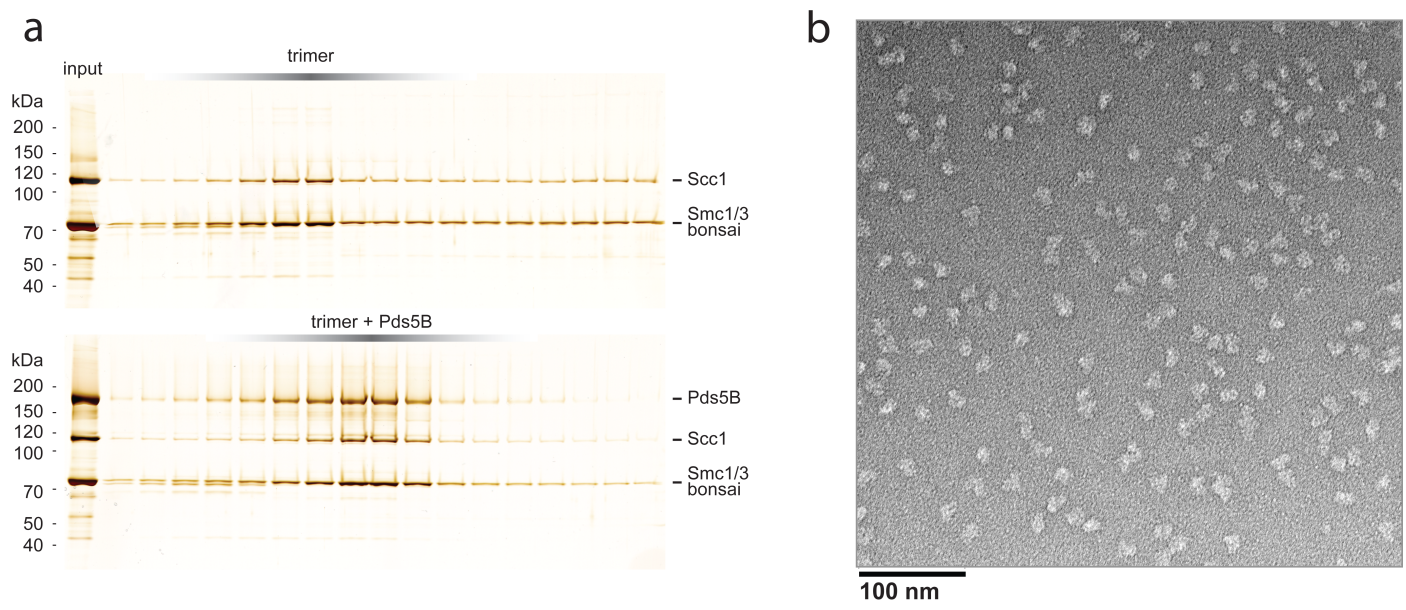

Figure 3.9: Biochemical Integrity of Pds5 - Bonsai Cohesin Trimer. a: A non-cross-linked gradient was fractionated and analysed in SDS-PAGE. Similarly as for Pds5 - bonsai cohesin tetramer a shift of the peak fraction to deeper fractions is shown in case of Pds5 binding. b: Electron micrographs show homogeneous and monodisperse sample of decent quality.

reference-free approach was chosen in order to be free of any reference bias. By resampling, 2D class averages were used to generate 5,000 initial 3D models, which were sorted by 3D classification. Thereby, a credible starting 3D model was obtained, which was improved by sorting. By supervised classification, the dataset was separated according to the Pds5 occupancy. Only 41.3\% (14,056 images) of the dataset was sorted for the 3D model with the expected Pds5 density. This subset of the dataset was used to refine the 3D model (3D model t5, see Figure 3.10a).

Overall, the 3D model t5 is similar to previous models. The resolution was determined as $35 \AA$ ( 0.5 criterion). At the top, a head segment with a central cavity is positioned. This is in accordance with previous $3 \mathrm{D}$ models ( $\mathrm{t} 2, \mathrm{t} 3$ and $\mathrm{t} 4$ ). The connection of the head segment to the two arms at the bottom is at the center of the front view. Between the two arms, a density is visible, which is very similar to the previously described Pds5 density (3D model t2). The main difference of this 3D model to previous 3D models becomes obvious in the back view. While all earlier 3D models ( $\mathrm{t} 1, \mathrm{t} 2, \mathrm{t} 3$ and $\mathrm{t} 4$ ) contain a clasp connecting the head segment to the two segments at the bottom, here, this density is largely missing. This result suggests that the clasp corresponds mainly to SA1. However, a smaller density (yellow in Figure 3.10d) is visible, connecting the head segment to the proposed Pds5 density. This density may be part of Scc1 or belong to Pds5. This assumption reasons on the fact, that Pds5 binding is known to be Scc1-dependent (Huis in 't Veld, 2013). Altogether, the described 3D model appears less defined than earlier described 3D models. Especially, the region where the coiled-coils are expected appears lower resolved. Further sorting of an increased dataset is expected to improve the 3D model similarly as in the case of Pds5 - bonsai cohesin tetramer. 


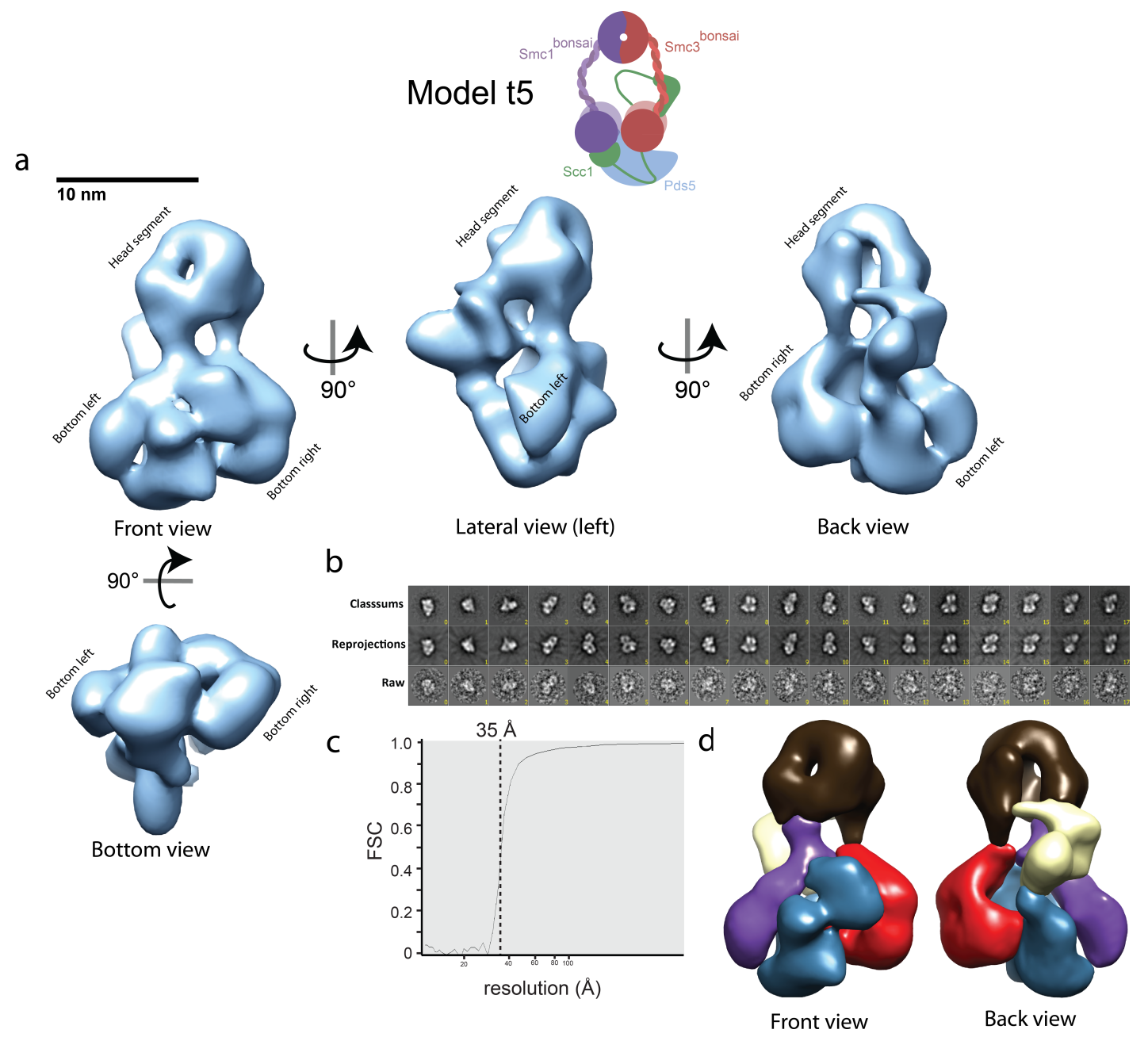

Figure 3.10: First Structural Insights into Pds5 - Bonsai Cohesin Trimer (t5). a: Illustration of the 3D model of Pds5 - bonsai cohesin trimer ( $\mathrm{t} 5$ ) in front view, lateral view (left), back view and bottom view. The 3D model is similar to previous models and similar densities can be identified. At the top, a head segment (brown) with the typical donut-shape is visible. A bottom left segment and a bottom right segment can be recognized, even though these densities are of limited definition. Between those densities, a density similar to the Pds5 density in $3 \mathrm{D}$ model $\mathrm{t} 2$ is visible. Interestingly, a smaller density (yellow in d) than the previously described clasp of 3D model t1-t4 is visible in the back view. With respect to the largely missing clasp, it is likely that the clasp mainly corresponds to SA1. The remaining small density (yellow in d) may correspond to parts of Scc1. Moreover, the presumable Pds5 density is connected to this small density. b: Selected projections, reprojections and related raw images of the 3D model are depicted. c: The structure was determined at a resolution of $35 \AA$ by FSC ( 0.5 criterion). d: The obtained 3D model t5 is manually segmented according to the proposed assignment: Head segment (brown), bottom left (purple), bottom right (red), proposed Pds5 density (blue). 
In summary, the first reference-free 3D model of Pds5 · bonsai cohesin trimer (t5) was obtained. By comparing this 3D model to the 3D model of Pds5 - bonsai cohesin tetramer (t2), a location for SA1 within bonsai cohesin is proposed.

\subsubsection{Assignment of Smc1 ${ }^{\text {bonsai }}$ Location}

We expected that cohesin has a pseudo-symmetric nature. This assumption is based on the presence of two distinct Smcs (Smc1, Smc3) in human cohesin. Each Smc is known to interact with another end of the asymmetric Scc1. This asymmetry strongly argues that Smc1 and the C-terminal domain of Scc1 form a distinct interface than Smc3 and the N-terminal domain of Scc1 ${ }^{1}$. While the interaction between the C-terminal domain of Scc1 and Smc1 has been known for long (Haering et al., 2004), the experimental evidence for this pseudo-symmetry and the distinct N-terminal Scc1 interaction was provided recently (Bürmann et al., 2013; Gligoris et al., 2014; Huis in 't Veld et al., 2014).

Symmetry is advantageous in structural biology ${ }^{2}$, albeit pseudo-symmetry may result in wrong averaging of similar but not identical features. To circumvent this problem, a symmetry-breaking tag was used for bonsai cohesin tetramer. This $2 x R F P \operatorname{tag}(53 \mathrm{kDa})$, is positioned at the C-terminus of Scc1, and it is expected to be in close proximity to the globular NBD of Smc1. The identification of this tag in a 3D model should allow to distinct Smc1 $1^{\text {bonsai }}$ from Smc3 $3^{\text {bonsai }}$.

The sample of $2 \mathrm{xRFP}$ bonsai cohesin tetramer prepared in negative stain was recorded using a Philips microscope (CM200) operated at $160 \mathrm{kV}$. The manual selection of $\sim 14,000$ particle images was conducted using CowPicker. Reference-free image processing did not yield satisfying results. The flexible nature of the tag may affect the image alignment. As the $2 \mathrm{xRFP}$ tag has a mass of $15 \%$ of the untagged bonsai cohesin tetramer, a strong flexibility of the tag may be deleterious. Such a flexibility is likely as the interaction with the tagged protein is loose. In a second approach, the obtained 3D model t3 (untagged bonsai cohesin tetramer) was used as reference on the 2xRFP-tagged dataset to possibly define the position of the $2 x R F P$ tag. Interestingly, in two repetitions of 3D classification, a 3D model (t6) was obtained, with an additional density (Figure 3.1.5b). The bulky density has a size matching the $53 \mathrm{kDa} 2 \mathrm{xRFP}$ tag, and it is located at the right bottom density in the front view. When the density threshold is changed, the density results in an elongated density, compared to the otherwise round density (t3). This result indicates that the Smc1 ${ }^{\text {bonsai }}$ NBD may be assigned to the right bottom density. Moreover, the previous

\footnotetext{
${ }^{1}$ Interestingly, Smc homodimers are formed in prokaryotes, while Smcs deviate in higher organism into distinct heterodimers. This may relate to the functional asymmetry in higher organism (e.g. the exit gate).

${ }^{2}$ An n-fold symmetry provides an $n$ times stronger signal.
} 
assumption that the Smc1/3 $3^{\text {bonsai }}$ globular NBD can be assigned to the opposing densities at the bottom (t1) in the front view is supported. Based on this concept, the NBD of Smc $3^{\text {bonsai }}$ can be assigned to the left bottom globular density. However, the additional density alone is not a confident validation as at low resolution and with strong sample heterogeneity a small density can be easily misinterpreted.
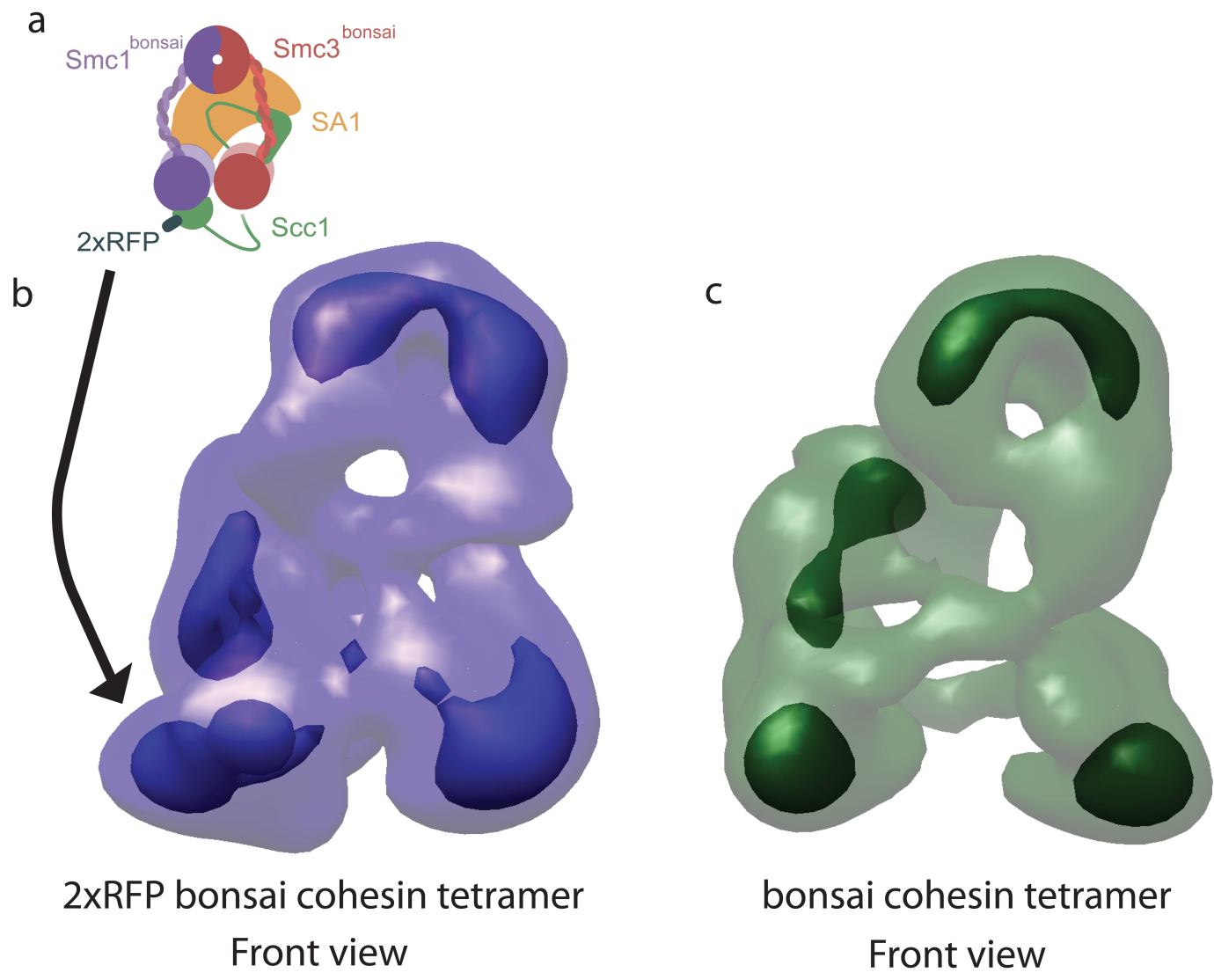

Figure 3.11: Detection of Smc1 ${ }^{\text {bonsai }}$. a: A dataset of bonsai cohesin tetramer where a $2 x R F P$ tag (dark green) is positioned at the C-terminus of Scc1 is used to distinguish Smc1 ${ }^{\text {bonsai }}$ from Smc3 ${ }^{\text {bonsai }}$. The C-terminus of Scc1 is known to be in proximity to Smc1. b: The shown 3D model t6 was calculated by using 3D model t3 as reference. For the illustration, two different thresholds are chosen and overlaid (transparent light blue and dark blue). At the left bottom a small but intense density is visible, which is proposed to correspond to the $2 \mathrm{xRFP}$ tag. The location of this density is in accordance with the interaction of the Scc1 C-terminus with the Smc1 NBD (compare image1.6). c: The 3D model of the untagged bonsai cohesin tetramer (t3) is shown in comparison, with two equivalently chosen thresholds (transparent light green, dark green).

In summary, by using a 2xRFP tag, an option to distinguish the Smc1 ${ }^{\text {bonsai }}$ NBD from the Smc $3^{\text {bonsai }}$ NBD is given. The identified additional density may correspond to the $2 x R F P$ tag.

\subsubsection{Wapl · Pds5 · Bonsai Cohesin Tetramer}

Very recently, a new cohesin complex containing Pds5 and Wapl was successfully purified by Dr. Pim Huis in't Veld. The complex was prepared and stabilised as explained previously, 
and homogeneous and monodisperse particles were visible after negative stain preparation (see Figure A.5.). It is known that Pds5 and Wapl directly interact. However, Pds5 binding depends on Scc1, while Wapl binding depends on SA1 (Huis in 't Veld, 2013). Wapl adds an additional mass of $150 \mathrm{kDa}$, so that a complex of $679 \mathrm{kDa}$ is formed. Thus, a generated 3D model should contain an additional density in proximity to Pds5 and SA1. Images were recorded and reference-free data processing was initiated. The image processing was performed by Jan Kaesler under supervision of the author of this work. Reference-free 2D processing of a dataset of Wapl - Pds5 - bonsai cohesin tetramer was initiated. The obtained class averages were compared to class averages of Pds5 - bonsai cohesin tetramer. Class averages were screened for an additional density at the expected position, and promising class averages were selected. The selected class averages were used for 3D reconstruction, either by angular reconstitution or by applying the angular information of the most similar projection images of the 3D model of Pds5 - bonsai cohesin tetramer. Thereby, intial 3D models were obtained (Figure A.6). Although these 3D models can still be refined, an overall similarity to the previous 3D models is given. It is very likely that the Wapl density can be assigned similarly as shown in the cases of Pds5 or of SA1.

\subsubsection{Validation of Cohesin Structures}

As outlined earlier, structural validation is currently a "hot topic" in EM. Validation standards are not as established as in X-ray crystallography, and wrong 3D models are still published due to missing standards. On the other hand, validation of a correct 3D model is difficult for some small heterogeneous samples as standard approaches can fail, even if a 3D model is credible. In the cases were 3D models of so far unknown structures are generated, a successful validation is important and common criteria need to be defined for the EM community.

\subsubsection{Internal Validation}

Applied references can easily bias a 3D model towards the reference, resulting in features mimicing the reference. Accordingly, images of random noise can be used to derive every wished 2D or 3D model. In the case of Pds5 - bonsai cohesin tetramer, it was possible to reproduce a random 3D model (dinosaur) as reference from the dataset by projection matching. Even if an additional classification step is included and angular reconstitution is performed, rudimentary features of a dinosaur model can be obtained (Figure 2.9). Therefore, a reference-free approach was chosen for bonsai cohesin tetramer, Pds 5 - bonsai cohesin tetramer and Pds5 · bonsai cohesin trimer. 
The presented 3D models (t1, t2 and t5) were obtained completely reference-free ${ }^{3}$. Thus, a confirmation of the reliability of the presented 3D models is given by the fact that $3 \mathrm{D}$ models of similar shape, with specific distinctions related to certain added or omitted factors (Pds5, SA1), were obtained. Moreover, by sorting a Pds5 · bonsai cohesin tetramer dataset, the compositional heterogeneity was used to obtain improved 3D models of bonsai cohesin tetramer ( $\mathrm{t} 3$ and $\mathrm{t} 4$ ). This makes the 3D models credible, despite their limited resolution.

Moreover, different regions of the Pds5 - bonsai cohesin tetramer structure have been subjected to density recovery with $\Delta$ reference models where the respective region was removed. Densities are removed manually by applying a Gaussian mask to obtain reference $\Delta$ 3D models. Successful recovery of a density confirms the density's authenticity. Four areas were selected for manual recovery: the proposed Pds5 density (a), both supposed Smc1/3 $3^{\text {bonsai }}$ NBDs (b,c) and a part of the backside clasp (d), here referred to as "finger". All four areas were fully recovered (Figure 3.12). In the case of the proposed Smc1 ${ }^{\text {bonsai }}$ and the "finger", projection matching was performed. In the case of Pds5 and Smc3 $3^{\text {bonsai }}$, a 3D classification recovered the removed densities.

\subsubsection{2 "Gold standard" Validation}

In X-ray crystallography $\sim 10 \%$ of a dataset which is not used for model building is used to define a validation factor, the $\mathrm{R}$-free value. In analogy, a second dataset of a new purified sample of Pds5 - bonsai cohesin tetramer was processed ab initio and reference-free. Image processing was performed by Jan Kaesler under supervision of the author of this work.

A new purification and preparation generated a sample of Pds5 - bonsai cohesin tetramer in negative stain. As described in 3.1.3.2, a dataset of $~ 50,000$ particle was obtained. This dataset was used in two different approaches. First, the original dataset was increased for refinement and heterogeneity evaluation. Second, the new dataset was subjected to $a b$ initio processing to obtain a new 3D model which can be correlated with the original 3D model of the previous dataset. 2D image processing was performed reference-free, yielding 1000 class averages of approximately 50 images per class. From the initial class average set, 284 class averages were selected as input for SIMPLE Prime. After 12 iterations, a 3D model converged, which was used for further reference-based 3D processing. Using several iterations of 3D classification, a 3D model which showed an overall similarity to previously processed 3D models of Pds5 - bonsai cohesin tetramer was generated (Figure 3.13). All

\footnotetext{
${ }^{3}$ Exceptions are the 3D models of $2 \mathrm{xRFP}$ bonsai cohesin tetramer (3D model t6) and Wapl Pds 5 - bonsai cohesin tetramer. The $3 \mathrm{D}$ models $\mathrm{t} 3$ and t4 were obtained by using a $\Delta 3 \mathrm{D}$ model (minus Pds5 density) as reference.
} 
a

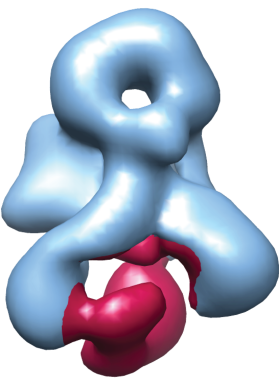

C

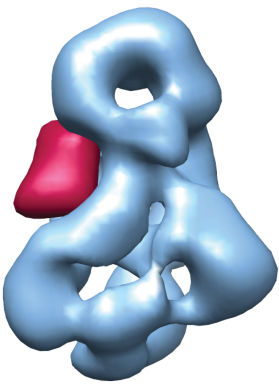

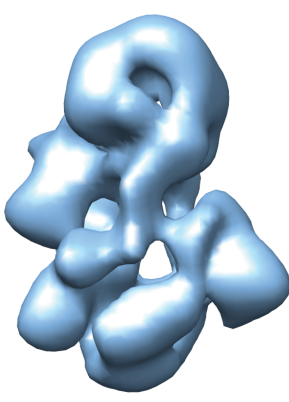

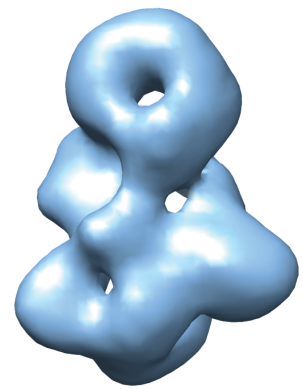

b
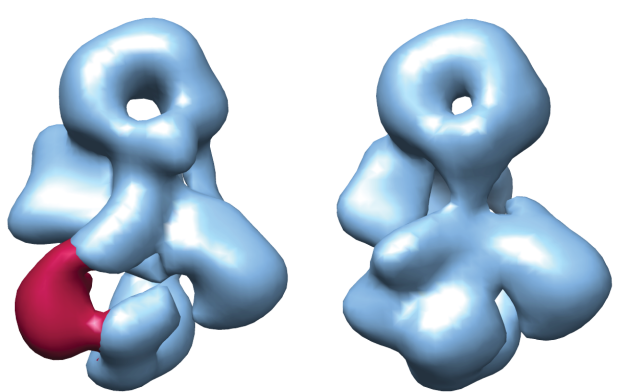

d
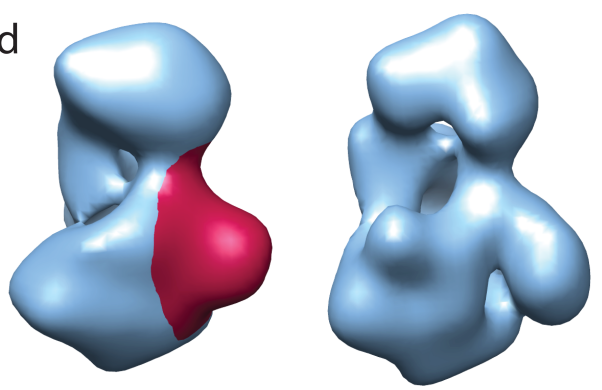

Figure 3.12: Density Recovery from $\Delta$ Reference Models for Density Validation. The recovery of a density which is removed in the reference model assures this density to be real. Reference bias can be avoided. Four different regions of Pds5 - bonsai cohesin tetramer were chosen to be removed and tested for recovery. a: The proposed Pds5 density (red) was removed and the delta model (left, blue) was used as reference for the dataset. An improved 3D model compared to the reference was obtained, containing the expected Pds5 density. b: The left bottom segment (proposed Smc1 ${ }^{\text {bonsai }}{ }^{\mathrm{NBD}}$ ) was removed (red) in the reference 3D model (left, blue). The density was recovered by projection matching (right). c: A part of the clasp was removed (red) in the reference 3D model (left, blue). The density was recovered by projection matching (right). d: The probable Smc $3^{\text {bonsai }}$ density (red) was removed in an early state of image processing. Accordingly, the used reference 3D model (left, blue) is not as defined as in a, b and c. However, the density of expected Smc $3^{\text {bonsai }}$ recovered and was improved compared to the reference $3 \mathrm{D}$ model (right). In b and c, different from a and $\mathrm{d}$, the generated $3 \mathrm{D}$ model did not outperform the input reference 3D model. This is reasoned in the fact that here projection matching was performed until the corresponding density reappeared (maximal three rounds). 
previously described densities (head, bottom left, bottom right, Pds5, clasp) recovered, albeit still very bulky. Accordingly, the correlation of the two 3D models was only 0.794 ( 1 would be identical). It is to be expected that overcoming limitations regarding the pseudo-symmetry and further sorting steps during image processing will improve the similarity between the derived 3D models. In summary, the obtained 3D model, albeit of still limited quality, seems to validate the presented 3D models.

\subsubsection{Tilt Validation}

Initially, tilt validation was performed using a negatively stained bonsai cohesin tetramer sample ${ }^{4}$. A tilt of $45^{\circ}$ was applied. The predefined image rotation of $-46^{\circ}$ and the image tilt of $45^{\circ}$ were experimentally confirmed by comparing $3 \times 3$ concatenated micrographs (tilted and non-tilted) using the in-house software CowPicker. Manual selection of 1500 tilt-pairs enabled the generation of a dataset with minimum stain edges, which are known to limit the performance of validation. Tilt validation was performed as described (Rosenthal and Henderson, 2003; Henderson et al., 2011b) within CowEyes. A correct 3D model should allow the calculated tilt angles to be similar to the experimentally defined tilt angles. In a tilt-pair parameter plot (TPPP), the calculated values are plotted and should cluster around the experimentally adjusted values. The size of the cluster relates to the accuracy and the reliability of the validation.

A second tilt validation concept, the rotational tilt validation, is based on the strategy of reconstructing a 3D model of the tilted images by applying the theoretical euler angles according to reference-based image alignment of non-tilted images. The similarity between the reconstructed 3D models of tilted and non-tilted data, expressed by a correlation value, determines the validity of the $3 \mathrm{D}$ model.

The initial 3D model t1 could not be validated in the TPPP, as no tilt-pair cluster was obtained (Figure 3.14b.1). Moreover, the generated 3D model in the rotational tilt validation of the tilted images remained bulky and the defined correlation value $\chi$ was low $(\chi=3.3)^{5}$ (Figure 3.14c).

Limitations due to negative stain preparation can be responsible for an unsuccessful validation. Therefore, a vitrified tilt-pair dataset of 200 tilt-pairs with a tilt of $45^{\circ}$ was recorded. The tilt-pairs were selected manually and the dataset was processed similarly as described for the negative stain dataset. In the TPPP, no cluster was identified (Figure 3.14b3). Additionally, the rotational tilt validation failed. Accordingly, neither a negative stain tilt-pair dataset nor a cryo tilt-pair dataset validated the current model.

\footnotetext{
${ }^{4}$ The same grid was used as for 3D model t1.

${ }^{5}$ Based on test models, a value of $\chi \geq 6$ is necessary for a successful validation (Kirves, 2014).
} 
a
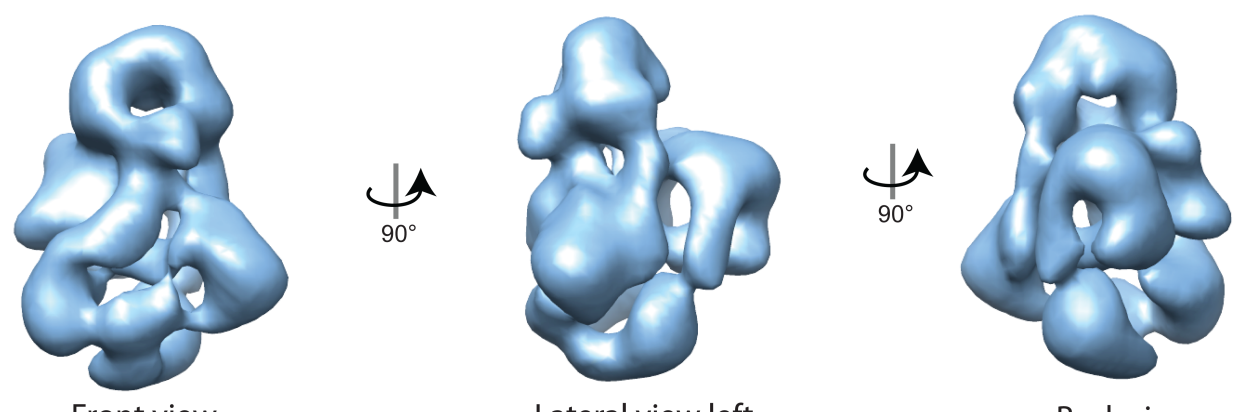

Front view

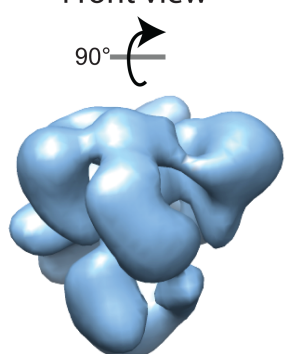

Lateral view left

Back view

b

\begin{abstract}
Bottom view
\end{abstract}

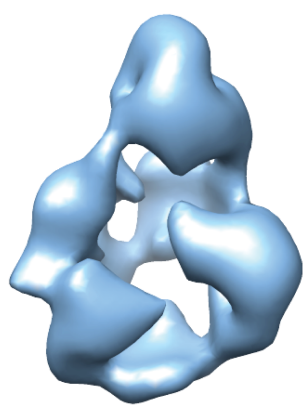

Front view

90ㄷ

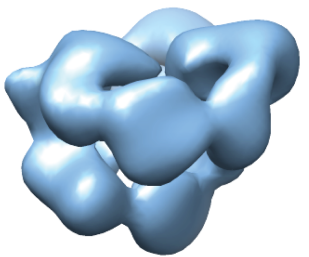

Bottom view
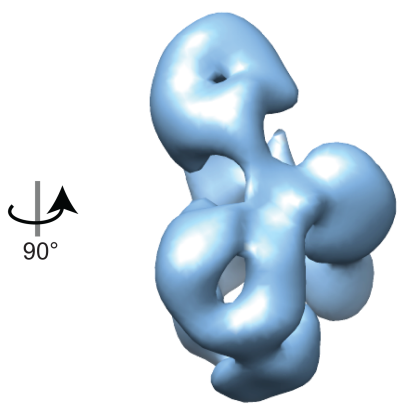

Lateral view left

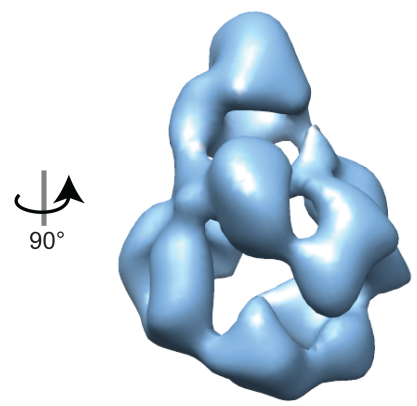

Back view

Figure 3.13: "Gold standard" Validation. To validate the obtained 3D model of the initial Pds5 - bonsai cohesin tetramer dataset, a new dataset was recorded. Reference-free image processing was performed to generate an independent $3 \mathrm{D}$ model. The similarity between both $3 \mathrm{D}$ models is a criterion for the structural validity. a: The obtained 3D model of the initial dataset is shown in front view, lateral view (left), back view and bottom view. b: The obtained 3D model of the gold standard validation is shown in equivalent views. A similarity between $\mathbf{a}$ and $\mathbf{b}$ is visible. While the $3 \mathrm{D}$ model shown in a could not be further improved with the used dataset, the 3D model in $\mathrm{b}$ is only preliminary. It is expected that further image processing and sorting may increase the similarity. The $3 \mathrm{D}$ model in $\mathbf{b}$ was obtained by Jan Kaesler under supervision of the author of this work. 
Tilt-pairs of a negative-stain dataset of the Pds5 - bonsai cohesin tetramer were used to validate the corresponding $3 \mathrm{D}$ model $\mathrm{t} 2$. Image recording was performed with a tilt of $45^{\circ}$. The tilt-pair dataset was subjected to a supervised classification step to remove the tilt-pairs assigned to the Pds5-free particles (63\%). As before, neither the TPPP (Figure 3.14b2) nor the rotational tilt validation could validate the 3D model. Jan Kaesler used a tilt-pair dataset of only $15^{\circ}$ which showed better clustering in TPPP and an improved correlation $(\chi=4.9)$ in the rotational tilt validation. In this context, it is important to consider that a lower tilt angle automatically yields a better result, while this does not imply a more successful validation. Systematic limitations due to image tilting are likely to be responsible for the inability to validate the 3D models. Datasets of small and heterogeneous particles are difficult to validate by this approach. 
a

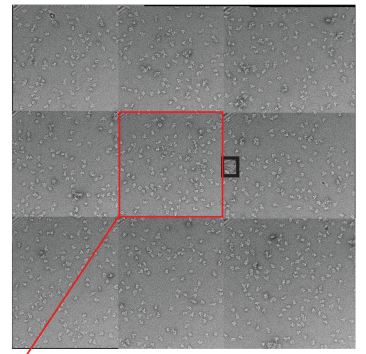

$0^{\circ}$ tilted Pds 5 bonsai cohesin tetramer

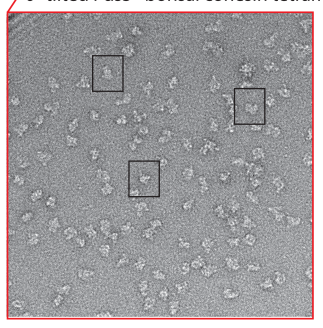

$\mathrm{C}$

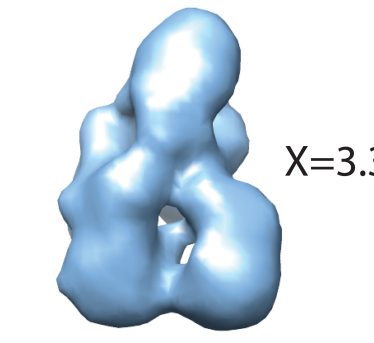

Non- tilted bonsai cohesin tetramer

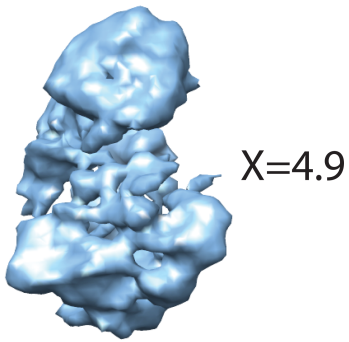

Non-tilted bonsai Pds $5 \cdot$ cohesin tetramer Tlted Pds $5 \cdot$ bonsai cohesin tetramer $15^{\circ}$
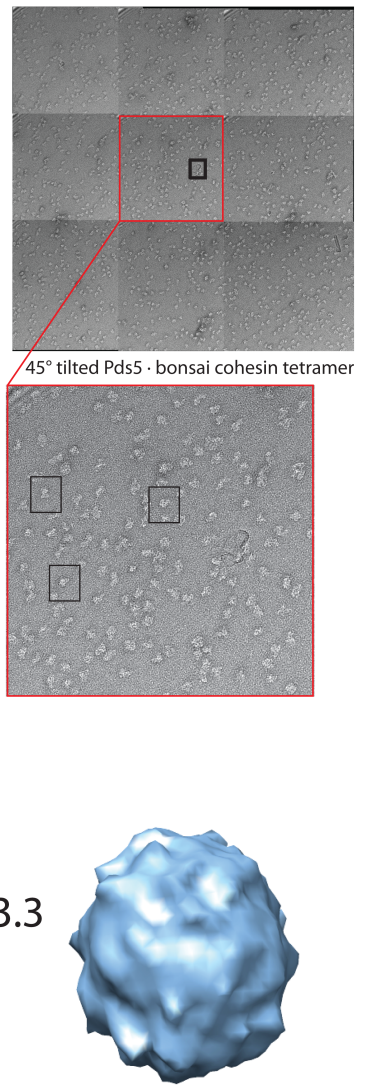

Tilted bonsai cohesin tetramer $\left(45^{\circ}\right)$

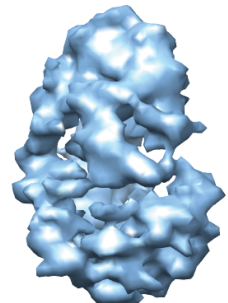

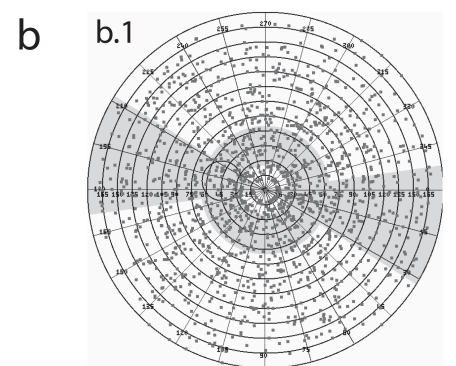

TPPP of negative stain bonsai cohesin tetramer $\left(45^{\circ}\right)$

b.2

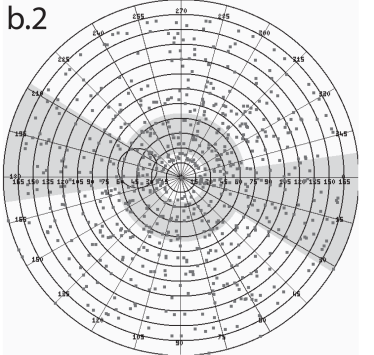

TPPP of negative stain Pds 5 . bonsai cohesin tetramer $\left(45^{\circ}\right)$

b. 3

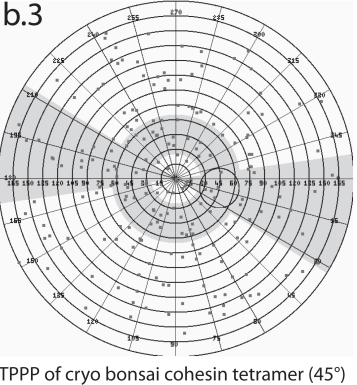

b.4

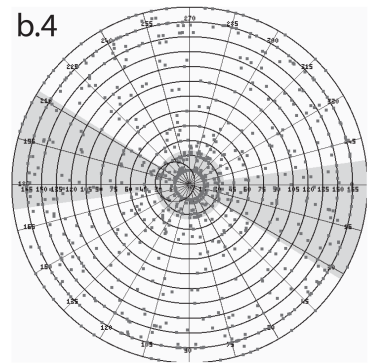

TPPP of Pds $5 \cdot$ bonsai cohesin tetramer $\left(15^{\circ}\right.$

Figure 3.14: Tilt Validation. a: Two corresponding 3x3-appended electron micrographs, with $\left(45^{\circ}\right)$ and without $\left(0^{\circ}\right)$ tilt are depicted. The black square highlights equivalent areas. In a magnified illustration equivalent areas are shown and three particle images are depicted. The apparent distance changes due to the applied tilt. b: Several obtained TPPP are shown: b.1 (negatively stained bonsai cohesin tetramer with $45^{\circ}$ tilt); b.2 (negatively stained Pds5 - bonsai cohesin tetramer with $45^{\circ}$ tilt); b.3 (vitrified Pds5 - bonsai cohesin tetramer with $45^{\circ}$ tilt); b.4 8negatively stained Pds5 - bonsai cohesin tetramer with $15^{\circ}$ tilt). In all cases, a circle highlights the area where the cluster is expected in case of a successful validation. However, none of the tested conditions validate the 3D model (b.1: 3D model t1, b.2: 3D model t2, b.3: 3D model t2, b.4: 3D model t2) of the respective complex. In the case of only $15^{\circ}$ tilt, an improved but not satisfying clustering was observed. Systematic limitations due to complex size, heterogeneity and pseudo-symmetry are expected to explain the unsuccessful validation attempt. c: Rotational tilt validation attempts of bonsai cohesin tetramer and Pds5 - bonsai cohesin tetramer. The tilted reconstructions were of limited comparability to the non-tiled reconstructions as expressed by low $\chi$ values. 


\subsection{Influence of Viomycin on 70S Ribosome Dynam- ics}

In the following section, the results regarding the effects of viomycin on the 70S ribosome dynamics will be summarised with a focus on 30S body rotation and tRNA dynamics. The observed impact of viomycin on the 70S ribosome dynamics provides novel structural insights into the inhibitory mechanism of this important antibiotic.

\subsubsection{Effects of Viomycin on Ribosomal 30S Body Rotation}

The 70S ribosome is known to undergo a counter-clockwise 30S body rotation from pre1 state to pre5 state and to switch back to the non-rotated state upon transition to the post1 state. This rotation, the so-called ratcheting, is loosely coupled to the tRNA transition from classical to hybrid states. The subsequent tRNA translocation correlates strongly with the attendant step to the non-rotated state of the 30S body (Fischer et al., 2010). Even more, this rotation is known to be essential for translocation (Horan and Noller, 2007). Dr. Niels Fischer showed previously that rotations of $-4^{\circ}$ to $13^{\circ}$ around a non-rotated ground state $\left(0^{\circ}\right)$ are detectable at $4^{\circ} \mathrm{C}$. The pivot point of this rotation is h27 (Fischer et al., 2010).

Viomycin is known to inhibit translocation more than 10,000 fold (Peske et al., 2004). However, the concrete action of inhibition is discussed controversially. Different approaches suggest either rotated or non-rotated states of a viomycin-inhibited 70S ribosomes. By $\mathrm{X}$-ray crystallography, viomycin-bound 70S ribosome structures were observed in the non-rotated state (Stanley et al., 2010). In contrast, FRET measurements defined a rotated state (Ermolenko et al., 2007; Cornish et al., 2008). Accordingly, it is not ensured if there is a relevant effect of viomycin related to the rotation state of the $30 \mathrm{~S}$ body. Therefore, the possible effect of viomycin on $30 \mathrm{~S}$ body rotation is studied systematically in this work.

By supervised classification, an EM dataset can be sorted according to features of the used reference 3D models, which are expected to be represented within the dataset. Here, 19 different modelled rotated states of the 30S body were used to sample the whole possible rotational space. The 30S body rotations, which were used as reference input, range from $-20^{\circ}$ to $25^{\circ}$ with $2.5^{\circ}$ intervals. The chosen angular sampling was shown to fulfil the requirements for adequate sorting. Moreover, very flexible regions were removed from the reference map by applying a Gaussian mask. These regions are the protruding L1 and L12 and the swivelling 30S head. As it is known that the dataset will contain a certain 
amount of vacant 50S subunits, a 50S reference map is added to remove these sole $50 \mathrm{~S}$ subunits.

Two datasets of vitrified pre-translocation complex (pre-TC) were recorded, one with added viomycin (referred to as +viomycin) and one without added viomycin (referred to as -viomycin). The addition of a concentration of $200 \mu \mathrm{M}$ viomycin was shown to guarantee a saturated viomycin binding (Peske et al., 2004). Previous experiments of $4^{\circ} \mathrm{C}$ or $37^{\circ} \mathrm{C}$ pre-incubated samples showed either reduced rotational space or a totally homogeneous rotational space, respectively (Fischer et al., 2010). In order to reduce the influence of an overlaid temperature effect, we decided to use pre-incubated pre-TC at intermediate temperature of $20^{\circ} \mathrm{C}$, showing a distinct profile of $30 \mathrm{~S}$ body rotation and being close to physiological conditions. Moreover, the $M g^{2+}$ concentration was adjusted to $3.5 \mathrm{mM}$.

After pre-processing, a dataset of 327,831 images was reduced to 279,217 images in the case of pre-TC + viomycin. For pre-TC -viomycin a dataset of 271,292 images was treated equivalently with remaining 227,617 images. Both datasets were subjected to a supervised classification according to the reference set (19 different states of $30 \mathrm{~S}$ body rotation and an vacant $50 \mathrm{~S}$ subunit). On the basis of CCC, every image is assigned to a certain reference. $13 \%$ of the images of pre-TC + viomycin and $17 \%$ of the images of pre-TC -viomycin were assigned to the vacant 50S subunit. By calculating the proportion of images assigned to each $30 \mathrm{~S}$ body rotation relative to all images assigned to $30 \mathrm{~S}$ body rotations between $-12.5^{\circ}$ and $17.5^{\circ}$, the dataset was normalised. The proportion of different $30 \mathrm{~S}$ body rotations was plotted (see Figure 3.15 and 3.16).

In Figure 3.15, the influence of viomycin is shown. Obvious are two visible maxima for both curves, at $0^{\circ} 30 \mathrm{~S}$ body rotation and at $7.5^{\circ} 30 \mathrm{~S}$ body rotation. However, the intensity of both maxima is interchanged between the viomycin-free and the viomycin-bound pre-TC condition. In the case of a pre-TC -viomycin, the $0^{\circ}$ maximum is higher than the $0^{\circ}$ maximum of a pre- $\mathrm{TC}+$ viomycin. In contrast, the $7.5^{\circ}$ maximum of the pre- $\mathrm{TC}$-viomycin is lower than in the case of pre-TC + viomycin. Overall, the most preferred conformation of the pre-TC -viomycin seems to be the non-rotated state $\left(0^{\circ}\right)$, while pre-TC +viomycin mostly prefers a rotated state $\left(7.5^{\circ}\right)$. In the pre-TC + viomycin, all states, especially the very negative rotations, are reduced in favour of the maximum from $2.5^{\circ}$ to $15^{\circ}$. This data provides clear evidence for the influence of viomycin on the rotation state of the pre-TC. While previous data suggested either rotated or non-rotated states (Ermolenko et al., 2007; Cornish et al., 2008; Stanley et al., 2010), the presented data indicates viomycin-bound pre-TC to sample both states, while rotational states are preferred. However, other factors may have also an effect on the preferred rotation state. In single molecule conditions, $\mathrm{Mg}^{2+}$ concentrations of $15 \mathrm{mM}$ are applied, where the 70S ribosome is stabilised to reduce subunit dissociation. Accordingly, previous FRET experiments may have been influenced 

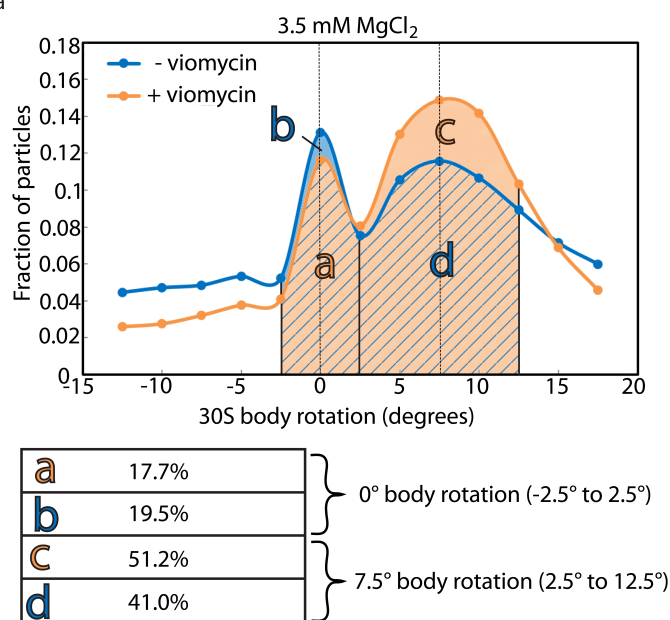
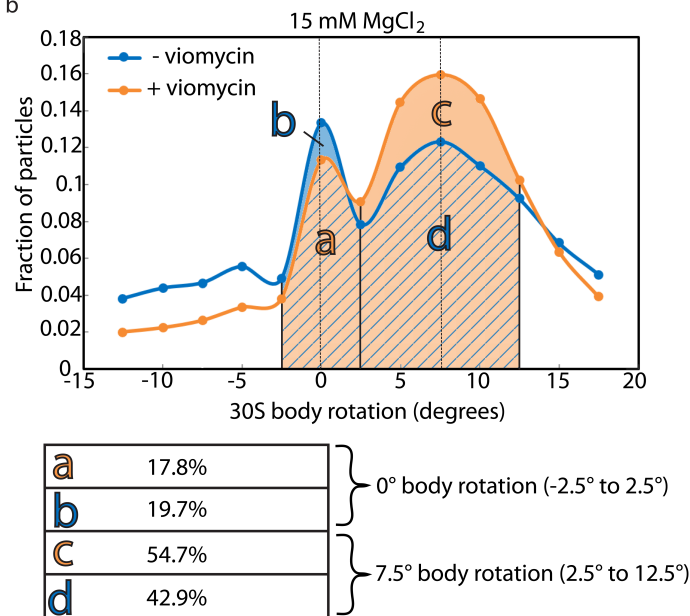

Figure 3.15: Viomycin affects the 30S Body Rotation. a: Images can be sorted for different rotational states of the $30 \mathrm{~S}$ body. This sorting is shown for a $70 \mathrm{~S}$ ribosome pre-TC $(3.5 \mathrm{mM} \mathrm{MgCl} 2)$ with and without added viomycin. The addition of viomycin shifts more $70 \mathrm{~S}$ ribosome pre-TCs to higher $30 \mathrm{~S}$ body rotated states (orange curve) compared to the sample without viomycin (blue curve). In both cases two maxima can be identified, a $0^{\circ}$ maxima and a $7.5^{\circ}$ maxima. When viomycin is added, the $0^{\circ}$ maxima is decreased and the $7.5^{\circ}$ maxima is increased. Below the diagram, the relative amount of particles in certain states is shown for both cases (proportion a,b,c,d). The described effect can be realised from the changed proportion of particles in the defined states $\left(0^{\circ}\right.$ maxima in a and $\mathrm{b}$ and $7.5^{\circ}$ maxima in c and d). b: Similarly as in a, the fraction of particles in certain 30S body rotations and high $\mathrm{MgCl} 2$ concentration is illustrated depending on viomycin addition. It is shown that an increased concentrations of $\mathrm{MgCl}_{2}(15 \mathrm{mM})$ has no effect on the $30 \mathrm{~S}$ body rotation. The curves in a and $\mathrm{b}$ are almost identical (A direct comparison is given in Figure A.7.). The proportion of particles assigned to different rotated states (proportion a,b,c,d) is very similar in the case of $3.5 \mathrm{mM} \mathrm{MgCl}_{2}$ and $15 \mathrm{mM} \mathrm{MgCl}_{2}$. 
by $\mathrm{Mg}^{2+}$ concentration. Therefore, we tested if the $\mathrm{Mg}^{2+}$ concentration influences the distribution, by repeating the experiment with a $\mathrm{Mg}^{2+}$ concentration of $15 \mathrm{mM}$. A dataset of 477,368 images was reduced to 254,938 images in the case of pre-TC + viomycin (15 mM $M^{2+}$ ) after pre-processing. For pre-TC -viomycin (15 mM $M^{2+}$ ), a dataset of 209,187 images was reduced by pre-processing to 80,585 images.

The distribution of the $30 \mathrm{~S}$ body rotation was equivalent to the case of $3.5 \mathrm{mM} \mathrm{Mg^{2+ }}$. Interestingly, the amount of vacant $50 \mathrm{~S}$ subunits was reduced compared to the $3.5 \mathrm{mM} \mathrm{Mg^{2+ }}$ condition, indicating that high $\mathrm{Mg}^{2+}$ concentration prevents $70 \mathrm{~S}$ ribosome disassembly. Moreover, in the pre-TC -viomycin, $13 \%$ of the images contained vacant $50 \mathrm{~S}$ subunits and, in the pre-TC + viomycin, $7 \%$ vacant $50 \mathrm{~S}$ subunits were identified. The plotted curves appear very similar to those in the case of $15 \mathrm{mM} M g^{2+}$ concentration. In summary, $M g^{2+}$ concentration does not influence the rotated state, which is caused by viomycin. The accurate reproducibility of the curves between different $M g^{2+}$ concentrations, indicates the reliability of these result. Altogether, it was shown that viomycin stabilises rotated states, while no effect of different $M g^{2+}$ concentrations can be observed.

\subsubsection{Effects of Polyamines on Ribosomal 30S Body Rotation}

As shown in Figure 2.1, the addition of polyamines can reduce the disassembly of the $70 \mathrm{~S}$ ribosome. Spermine shows the strongest effect. However, the naturally occurring polyamines in E. coli are spermidine and putrescine. Instead of testing the potential strongest effect of spermine, it was decided to conduct cryo EM with spermidine and putrescine.

The concentration of spermidine $(1 \mu \mathrm{M})$ and putrescine $(8 \mu \mathrm{M})$ was increased compared to earlier experiments to see a clear effect (see Figure 2.1). A $M g^{2+}$ concentration of $15 \mathrm{mM}$ was used. After pre-processing, 306,972 particle images remained. This dataset was subjected to a equivalent reference sorting according to $30 \mathrm{~S}$ body rotation and is shown in comparison with the $15 \mathrm{mM} \mathrm{Mg}^{2+}$ pre-TC data (-viomycin and + viomycin). Two maxima are visible at equivalent rotation states as for previous data $\left(0^{\circ}\right.$ and $\left.7.5^{\circ}\right)$ (Figure 3.16). Interestingly, the relative number of particles plotted against 30S rotation state seems to represent an intermediate state between the viomycin-free pre-TC and the viomycin-bound pre-TC. The relative number of particles in the rotated state is smaller than in the viomycin-bound pre-TC, but higher than in the viomycin-free pre-TC. Accordingly, very high concentrations of the polyamines spermidine and putrescine can induce a rotated ribosomal state similar to viomycin.

However, in the case of viomycin, only one molecule is bound to a specific site between $\mathrm{h} 44$, H69 and the A-site anticodon stem (Stanley et al., 2010). This one molecule modulates the 
a

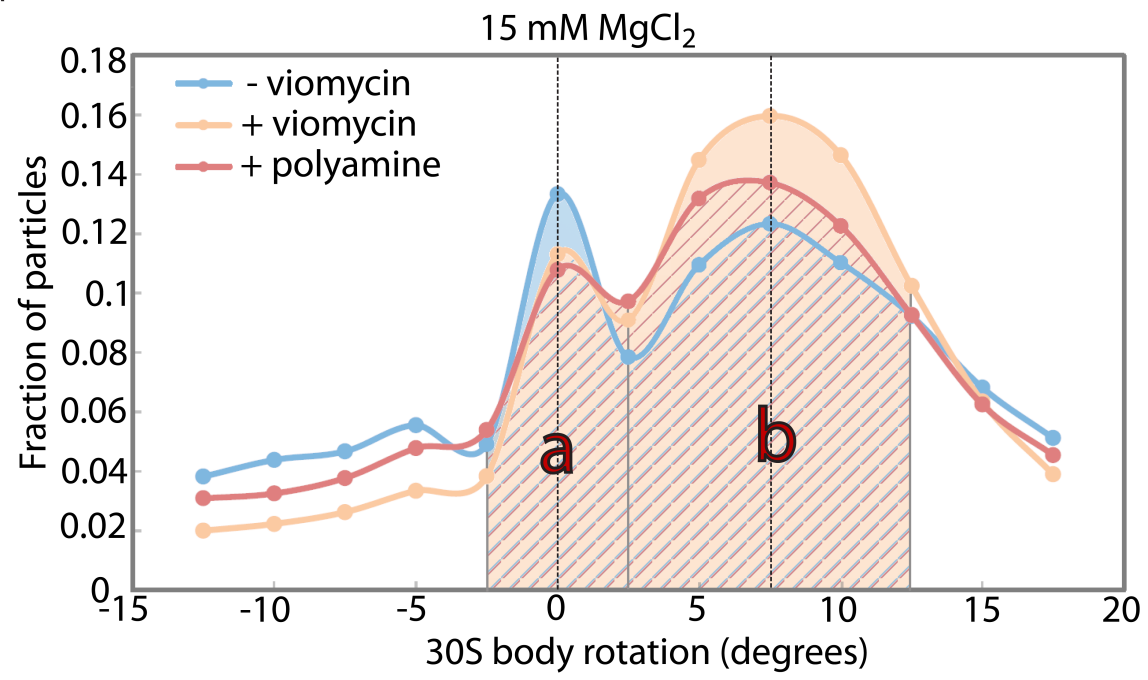

b

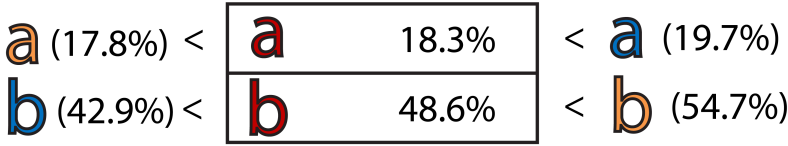
$0^{\circ}$ body rotation $\left(-2.5^{\circ}\right.$ to $\left.2.5^{\circ}\right)$
$7.5^{\circ}$ body rotation $\left(2.5^{\circ}\right.$ to $\left.12.5^{\circ}\right)$

Figure 3.16: High Polyamine Concentration influence the 30S Body Rotation. a: The effect of a high polyamin concentration on $30 \mathrm{~S}$ body rotation is compared to the effect of viomycin. It is shown that with polyamines a similar, albeit weaker effect can be induced as by viomycin addition. High $30 \mathrm{~S}$ body rotated states are favoured. b: The effect of polyamines is expressed by the changed proportions of $0^{\circ} 30 \mathrm{~S}$ body rotation (proportion a) and $7.5^{\circ} 30 \mathrm{~S}$ body rotation (proportion b). As shown, an intermediate state between the non-added and the added viomycin is obtained.

rotational state of the substantial bigger 30S body specifically, while only the collaborative action of many polyamine molecules stabilise the rotated state of the $70 \mathrm{~S}$ ribosome $30 \mathrm{~S}$ body.

\subsubsection{Influence of Viomycin on tRNA Dynamics}

Regarding the strong effect of viomycin on the 30S body rotation and the direct interaction with the A-site anticodon stem, an effect of viomycin on tRNA hybrid state formation is expected. Previous data suggested contradictory effects of viomycin for tRNAs. Complete tRNA classical states (A/A \& P/P) (Stanley et al., 2010), partly tRNA hybrid states (A/A \& P/E) (Pan et al., 2007) and complete tRNA hybrid states (A/P \& P/E) (Ermolenko et al., 2007; Cornish et al., 2008) were proposed. Cryo EM allows to sort the data on tRNA level and to define the influence of viomycin on the tRNA state. Previously, similar sorting strategies on tRNA states were shown to be successful (Fischer et al., 2010).

Various sorting strategies were applied on the presented datasets. In the following section, two different approaches to address the question related to the effect of viomycin on the tRNAs will be outlined and related results will be shown. 
Initially, the viomycin-bound pre-TC dataset with $3.5 \mathrm{mM} \mathrm{Mg}^{2+}$ was tested. Two subsets according to the related two maxima at $0^{\circ}$ and $7.5^{\circ} 30 \mathrm{~S}$ body rotation state were analysed. Two populations of each dataset were isolated ranging from $0^{\circ}$ to $5^{\circ}$ (fraction 1 ) and $5^{\circ}$ to $10^{\circ}$ (fraction 2). The isolated fractions can be aligned against a 50S subunit and be refined. Thereby, no very well-defined tRNA states are to be expected, as it has been shown that 30S body rotation and tRNA states are only loosely coupled (Fischer et al., 2010). Here, a resampling approach was performed (see ??). 2D images assigned to specific rotation states (fraction 1 or fraction 2) were used. Each 2D image set was randomly divided into subsets of $\sim 50$ images per subset and individual 3D models were reconstructed for each subset. Thereby, a few thousand 3D models (one 3D model per subset) were obtained for each set. Each set of 3D models was subjected to focused 3D MSA. Briefly, an elliptic area corresponding to the tRNAs and surrounding ribosomal areas was isolated by applying a Gaussian mask and focused 3D MSA was performed on this region. Accordingly, similar tRNA states were pooled and the signal-to-noise ratio of each 3D model was improved. Up to 500 3D models remained for each set ( $\sim 50$ 3D models per 3D class). These 3D models were inspected manually and few were selected on the basis of the most defined tRNAs. Accordingly, 14 3D class averages of fraction 1 and 17 3D class averages of fraction 2 were selected. In both cases, they mostly resembled $\mathrm{A} / \mathrm{P} \& \mathrm{P} / \mathrm{E}$ state, $\mathrm{A} / \mathrm{A} \& \mathrm{P} / \mathrm{E}$ state or empty \& P/E state. Still, the tRNA densities were of limited definition (Figure 3.17a). Interestingly, no classical state $(\mathrm{A} / \mathrm{A} \& \mathrm{P} / \mathrm{P})$ could be defined. The datasets (fraction $1 \& 2$ ) were refined for each of those $3 \mathrm{D}$ class averages to improve the tRNA densities. However, the tRNAs densities did not improve. Therefore, a different strategy to sort the data was chosen.

With available references of different tRNA states within the ribosome, a dataset can principally be sorted in a reference-based approach. In case of the ribosome, by combining cryo EM data and crystal data for MD simulation, the transition from pre1 to post4 state was described on pseudo-atomistic level, revealing functional motions on a level of detail which was previously inaccessible (Bock et al., 2013). Accordingly, for every tRNA state, a reference $3 \mathrm{D}$ model is accessible, which can be used for supervised classification and subsequent refinement. The viomycin-bound pre-TC dataset with $15 \mathrm{mM} \mathrm{Mg}^{2+}$ was used for initial trials. Reference 3D models of pre1a, pre1b, pre2, pre3, pre4, pre5a and pre5b were transformed into density maps, modified and size-adjusted (Figure 3.17b). In the reference 3D models, the flexible L1 and L12 were removed. The reference 3D models were down-filtered to $15 \AA$ to prevent high-resolution bias. The refined 3D models were used for another iteration of sorting and refinement, while tRNA positions stabilised. As shown, the fraction of particles is plotted against specific tRNA states $3.17 \mathrm{c}$ ). In the viomycin-bound pre-TC, two maxima are generated: one small maximum at the pre1 state and one high maximum at the pre4 to pre5 state. Accordingly, it seems as if there is a 


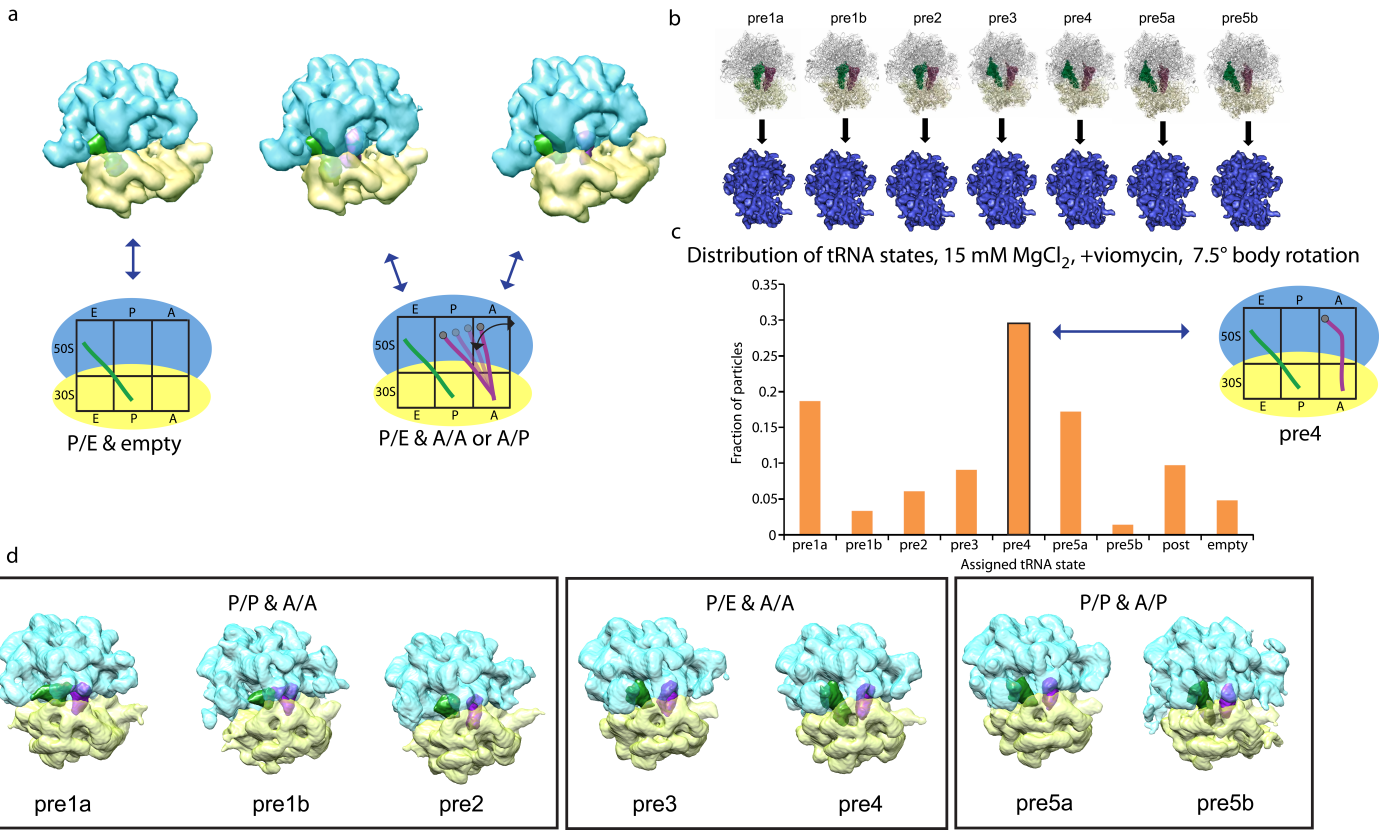

Figure 3.17: Influence of Viomycin on tRNA Dynamics. a: Images were sorted by 3D MSA for different tRNA position. Three representative 3D models of the 70S pre-TC with different tRNA states are shown. The tRNAs remain bulky and could not be further improved. However, all obtained 3D models showed tRNAs similar to one of the three shown states, which were assigned to $\mathrm{P} / \mathrm{E} \&$ empty and $\mathrm{P} / \mathrm{E} \& \mathrm{~A} / \mathrm{A}$ or $\mathrm{P} / \mathrm{E} \& \mathrm{~A} / \mathrm{P}$ states. The $\mathrm{A}$-site tRNA was not defined enough to distinguish between classical tRNA A/A and hybrid tRNA A/P states. b: Atomistic maps of pre1a to pre5b tRNA states are transformed into EM densities. Here, this is shown only exemplary. c: In a reference-based approach, these different references are used to sort a $70 \mathrm{~S}$ pre-TC dataset (15 $\mathrm{mM} \mathrm{Mg}^{2+}+$ viomycin) for tRNA states. The images were divided into different tRNA subsets and 3D models were obtained by projection matching. These 3D models were used for a second iteration of sorting, where the illustrated tRNA profile was obtained. Interestingly, by far most particles where assigned to a pre4 tRNA state $(\mathrm{P} / \mathrm{E} \& \mathrm{~A} / \mathrm{A})$. Moreover, many pre1a tRNA states $(\mathrm{P} / \mathrm{P} \& \mathrm{~A} / \mathrm{A})$ and pre5 tRNA states $(\mathrm{P} / \mathrm{E} \& \mathrm{~A} / \mathrm{P})$ were identified. With a tRNA distribution profile of a viomycin-free 70S pre-TC, a direct comparison will be possible to analyse the effect of viomycin on tRNA level. $\mathbf{d}$ : The 3D models of the first iteration reconstruction of $\mathrm{c}$ are shown. More distinct states than in a could be defined. The hybrid tRNA states (pre3-pre5) are better defined than the classical tRNA state. This is possibly a sign for preferred tRNA hybrid state formation. 
small amount of particles in classical state $(\mathrm{A} / \mathrm{A} \& \mathrm{P} / \mathrm{P})$, while most of the particles are in a tRNA hybrid state (Pre4: A/A \& P/E or Pre5: A/P \& P/E). This data is in accordance to the 3D MSA results 3.17a), where the dataset was mostly attributed to pre3 to pre 5 state. Nevertheless, there are differences which will be discussed below (see Chapter 4.2). Altogether, preliminary results indicate viomycin-bound ribosomes predominantly in tRNA hybrid states (Pre3-Pre5). 


\section{Chapter 4}

\section{Discussion}

A scientist's aim in a discussion with his colleagues is not to persuade, but to clarify.

— Leó Szilárd

\subsection{Cohesin Model Building and Interpretation}

In this work, varying cohesin complexes were used to comprehend the topology of a engineered bonsai cohesin complex. In the following section, the different 3D models which were obtained during this work will be compared and discussed. We expected that, by systematically omitting or adding certain subunits (Pds5, SA1) and subsequent comparison of the derived 3D models, it is possible to assign each subunit to a certain region.

Biochemical sample preparation was shown to be successful and sample of high quality fulfilling the requirements for single particle EM was obtained. All purified samples were shown to form isomorph and homogeneous complexes with little aggregation, as visible in electron micrographs. Moreover, each complex was shown to generate class averages of adequate quality for 3D processing. Finally, 3D models of the bonsai cohesin tetramer (t1, t3 and t4), the Pds5 - bonsai cohesin tetramer (t2) and the Pds5 - bonsai cohesin trimer ( $\mathrm{t} 5$ ) were obtained. A $3 \mathrm{D}$ model of $2 \mathrm{xRFP}$ bonsai cohesin tetramer ( $\mathrm{t} 6$ ) was used to distinguish Smc1 ${ }^{\text {bonsai }}$ from Smc $3^{\text {bonsai }}$ by reference-based image processing. However, no 3D model exceeds a resolution of $\sim 27 \AA$ ( 0.5 criterion). It is assumed that the overall heterogeneity within the dataset was limiting in obtaining higher resolved 3D models.

\subsubsection{Structural Conclusions on Bonsai Cohesin}

It was intended to identify Pds5 within the Pds5-bound bonsai cohesin complexes. In Figure 4.1a, the corresponding 3D model, t3 (bonsai cohesin tetramer) and t2 (Pds5 - bonsai cohesin tetramer) are compared side by side. Based on the difference between both models, 
Pds5 was assigned to a curved additional density at the bottom of and partly between Smc1/3 $3^{\text {bonsai }}$ NBDs. The density seems to reach from the center of the back view to almost the center of the front view. Regarding the assumed Pds5 position, the 3d models of Pds5 - bonsai cohesin trimer and Pds5 - bonsai cohesin tetramer were in accordance (t2, t5). Both 3D models (t2, t5) contain a density between the assumed Smc ${ }^{\text {bonsai }}$ NBDs, which is not existing in any 3D model of bonsai cohesin tetramer ( $\mathrm{t} 1, \mathrm{t} 3, \mathrm{t} 4)$. Moreover, the Pds5 density was shown to be real, as it can be recovered from the $\Delta 3 \mathrm{D}$ model in which the density is removed (Figure 3.12). Both Pds5-bound complexes were shown to contain a high amount of compositional heterogeneity regarding the Pds5 occupancy (Figure 3.7a). Only $\sim 40 \%$ of the particles in the sample were identified to be bound to Pds5. The determination of the precise ratio of Pds5-free and Pds5-bound particles depends on the resolution of the used 3D model. The low resolution may still limit the significance of the defined ratio. However, the presence of a high proportion of Pds5-free particles can not be neglected. The successful reconstruction of 3D models t3 and t4 matching the structure of 3D model t1 provides the evidence of Pds5-free complexes within the sample. Interestingly, the sorted data for the Pds5-free fraction allowed the reconstruction of improved 3D models for bonsai cohesin tetramer compared to the initial 3D model t1 (Figure 3.7b/c). Both datasets are similar in size (3D models t3 and t4: 31,309 2D images vs. 3D model t1: 33,500 2D images), so that this aspect seems to be negligible. Possibly, due to improved structural knowledge, the manual selection of class averages was improved in case of the 3D models t3 and t4. Additionally, the rigorous pre-processing step, removing many images, may be a reason for the improved 3D models t3 and t4.

By comparison of the 3D models t2 (Pds5 - bonsai cohesin tetramer) and t5 (Pds5 - bonsai cohesin trimer), it is assumed that SA1 is located within the clasp density (Figure 4.1b). As most of the clasp is missing in 3D model t5, it seems credible that the clasp corresponds to SA1. Still, a smaller density is visible on the backside of 3D model t5, which is connected to the defined Pds5 density. It is supposed that this small density corresponds not to SA1 but to a different subunit, possibly to a part of Scc1 or a part of Pds5. With respect to the available SA2 X-ray structure bound to a Scc1 fragment and the cross-linking data showing interactions between SA1 and Scc1, it seems likely that the smaller density corresponds to Scc1. Additionally, the omitted SA1 may result in a conformational change, so that the backside area appears different. However, a more detailed interpretation remains difficult due to the low resolution of the map. The high amount of flexibility of the density assigned to SA1 prevents the demarcation of the assumed SA1 from other densities in proximity. It may be that SA1 maintains a relatively high flexibility when bound to cohesin, allowing it to form varying conformations. Different from the described Pds5 density, it is not yet tested if the proposed SA1 density can be recovered when removed from the reference 3D model. Only the previously named "finger", which partly corresponds to the supposed 
SA1, was shown to recover (3.1.7.1). Further image processing is needed to achieve a similar definition of the clasp density as it was possible in case of Pds5.

Initial reference-free reconstructions of 2xRFP-tagged cohesin failed, which was reasoned in the flexibility of the tag. The tag is positioned at the C-terminus of Scc1. Regarding the high amount of the overall heterogeneity, an additional tag, corresponding to $15 \%$ of the total complex mass, may complicate a successful 3D model generation. The first reliable 3D model t1 of cohesin was generated for the tag-free cohesin. This suggested that the tag may be limiting in reference-free 3D model generation. However, the available 3D models was used for the $2 \mathrm{xRFP}$-tagged data in a reference-based strategy. By using the tetrameric $3 \mathrm{D}$ model $\mathrm{t} 3$ as reference, the reconstruction of a 3D model t6 was possible. In this 3D model, a small density fitting the size of a $53 \mathrm{kDa}$ tag became visible, which is not present in the used reference 3D model. Moreover, no further 3D model of non-tagged datasets showed a similar density at this position. In this context, it needs to be noted that the crystal structure of a C-terminal Scc1 fragment bound to Smc1 is in accordance with the proposed position of the $2 \mathrm{xRFP}$ tag in the shown 3D model t6 (Figure 1.6). Still, the limited resolution and an observed flattening on the tagged structure make it difficult to define if the observed density unequivocally relates to the included tag. Here, this density is assumed to represent the tag at the C-terminus of Scc1 in proximity to the assumed Smc1 ${ }^{\text {bonsai }}$ density, but stronger results are needed for hard evidence.

The 3D model t4 of bonsai cohesin tetramer represents the most detailed structure of all shown cohesin 3D structures in this work. The resolution is calculated as $\sim 27 \AA(0.5$ criterion). The interconnecting arms which are proposed to correspond to the coiledcoils cross each other. This possible X-shape implies that the left part of the assumed hinge belongs to the right Smc1 $1^{\text {bonsai }}$ NBD (bottom right), whereas the right part of the hinge belongs to the left Smc $3^{\text {bonsai }}$ NBD (bottom left). Earlier 3D models failed to show reasonable densities of the interconnecting arms. However, this potential X-shape of the coiled-coils in bonsai cohesin does not necessarily exist in case of the full-length cohesin. Another finding in this 3D model relates to the head segment. In all 3D models (apart from the initial 3D model t1), this head segment contains a central cavity (donut shape). In 3D model t4, the head segment appears most defined and the crystal structure of the hinge domain fits in form and size (Figure 3.7e). Considering the described heterogeneity in the dataset, the details of this 3D model need to be interpreted carefully. So far, it cannot be assured that the described observations related to 3D model t4 are correct. Further image processing is needed to assure the correctness of the proposed interpretation.

$\mathrm{ATP} \gamma \mathrm{S}$ was added in each preparation, as it was shown to stabilise bonsai cohesin tetramer (Figure 3.3c). It was supposed that the Smc1/3 $3^{\text {bonsai }}$ NBDs would be connected by the bound ATP $\gamma$ S. Similarly, in the crystal structure of Smc1 NBD homodimers (PDB:1W1W [yeast]), the two NBDs are connected to each other. However, the regions related to 
Smc1/3 $3^{\text {bonsai }}$ NBDs of all obtained 3D models are in proximity but not connected. An explanation is that in the case of crystallised Smc1 NBD homodimers a concentration of $1 \mathrm{~m} \mathrm{M} \mathrm{ATP} \gamma$ was used, while for all models in this work only $183 \mu \mathrm{M} \mathrm{ATP} \gamma$ was applied. Accordingly, the complex may have been undersaturated and only a fraction of the complexes was bound to ATP $\gamma$. In this context, a study from 2012 is of interest, which showed an ATP-dependant conformational change, bringing the NBD of a Rad50 dimer into a closed and condensed state (Möckel et al., 2012). Moreover, in 2015 it was

shown that Smc NBD engagement can be induced by ATP addition (Soh et al., 2015). In both cases higher concentrations of ATP $(1 \mathrm{mM})$ were added than in this work. Low ATP concentrations may be a further origin of flexibility within the complex. In the case of Pds5 - bonsai cohesin tetramer and Pds5 - bonsai cohesin trimer, the Pds5 density is partly in-between the Smc1/3 $3^{\text {bonsai }} \mathrm{NBD}$. It is not yet known if this is related to functional aspects of Pds5, and if Pds5 may prevent direct Smc1/3 NBD interaction.

In case of the bonsai cohesin tetramer dataset, the 3D model appeared elongated, which was attributed to an anisotropic angular distribution of images in the euler plot (Figure 3.4b). This can be a sign of preferred orientation of a complex on the carbon film. However, the improved 3D models of bonsai cohesin tetramer ( $\mathrm{t} 3$ and $\mathrm{t} 4$ ) did not show this elongation. Moreover, for Pds5 - bonsai cohesin tetramer, an homogeneous angular distribution was defined. Accordingly, this angular anisotropy may be a result of image sorting in the initial dataset. If there was a preferred orientation of the complex on the carbon film, the same elongation should have been identified for $3 \mathrm{D}$ models $\mathrm{t} 3$ and $\mathrm{t} 4$ as well, which was not the case. Thus, the elongation in $3 \mathrm{D}$ model $\mathrm{t} 1$ seems to be an artefact by initial image processing.

\subsubsection{Validation and Heterogeneity}

As shown in (3.1.7.3), the classical tilt-pair validation based on TPPP failed. Neither by using tilt-pairs from negative stain data nor vitrified data, the applied 3D models could be validated. Stain artefacts (stain edges) and sample flattening can be limiting in negative stain-prepared samples, whereas charging and low contrast may limit the successful validation of vitrified samples. Image tilting can be problematic, as these limitation are further increased. None of the used preparation techniques showed an improvement in the TPPP, although manual selection of tilt-pairs ensured that optimal images were chosen, while images with obvious stain edges (negative stain sample) and artefacts by charging (vitrified data) were discarded. The increased size of the Pds5-bound sample did not allow to improve the tilt validation. Tilt-pairs of both complexes (bonsai cohesin tetramer and Pds5 - bonsai cohesin tetramer) failed to validate the corresponding 3D models. In the case of Pds 5 - bonsai cohesin tetramer, a supervised classification was 
a

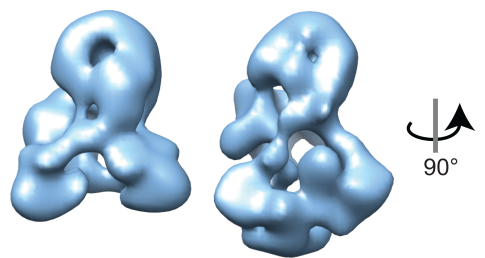

Front view

$90^{\circ} \mathcal{C}$

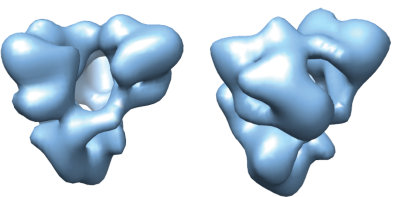

Bottom view

b

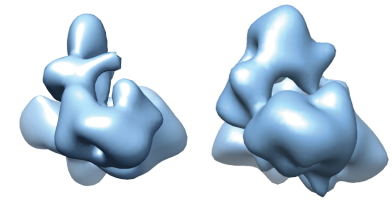

Top view

$90^{\circ} \mathrm{C}$

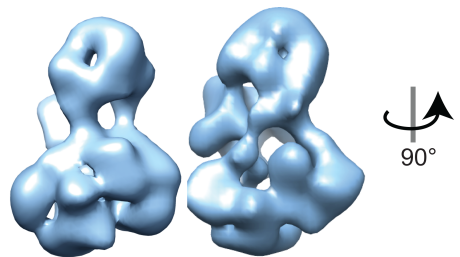

Front view

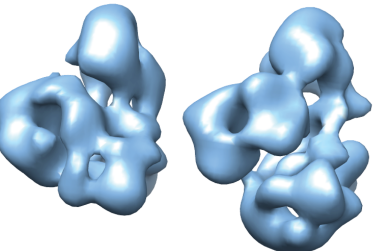

Lateral view (left)

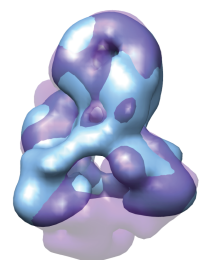

Overlay front view

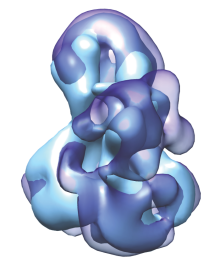

Overlay back view

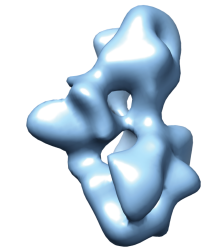

Lateral view (left)

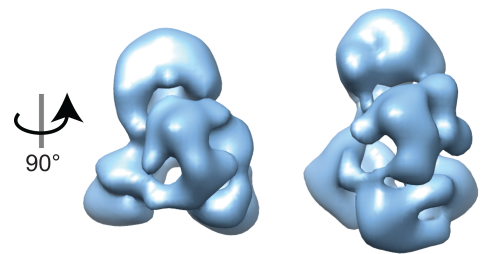

Back view

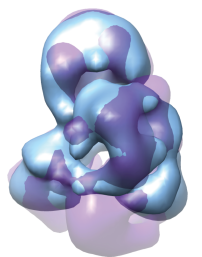

Overlay back view

Figure 4.1: Side by Side Comparison of distinct Bonsai Cohesin Structures. a: The 3D model of bonsai cohesin tetramer (t3) and of Pds5 - bonsai cohesin tetramer (t2) are compared. Additionally, an overlay of the front view and back view is shown (3D model t2: transparent purple), highlighting the curved Pds5 density as the main difference. b: The 3D model of Pds5 - bonsai cohesin trimer (t5) and of Pds5 - bonsai cohesin tetramer (t2) are compared. Additionally, an overlay of the front view and of the back view is shown (3D model $\mathrm{t} 2$ : transparent purple). The main difference is defined by the missing part of the backside density proposed to correspond to SA1. 
used to remove all tilt-pair images which correspond to Pds5-free complexes. Thereby, the compositional sample heterogeneity could be reduced. However, the clustering in the TPPP did not improve. By using tilt-pairs of only $15^{\circ}$ tilt angle, the clustering within the TPPP improved. Nevertheless, by lowering the tilt angles, the reliability of the tilt validation is decreased as well. Thus, this improved clustering cannot be evaluated as an improved validation. Moreover, the rotational tilt validation failed to validate the 3D models, as the generated 3D models from the tilted data appeared mostly unstructured and noisy and showed only low correlation. This was independent from the used dataset (bonsai cohesin tetramer and Pds5 - bonsai cohesin tetramer). In all cases, the correlation value was $<6$, which was defined as the minimum correlation value to declare a $3 \mathrm{D}$ model as credible. In the case of $15^{\circ}$ tilt-pairs, a correlation value of $X=4.9$ was defined, which is so far the highest correlation. It is assumed that there are systematic reasons for the inability to validate the current 3D models in a tilt-pair approach. It may be that a combination of parameters prevents the tilt validation of the $3 \mathrm{D}$ models. Especially complex size, compositional and conformational heterogeneity and pseudo-symmetry are limiting factors for tilt validation. All of these parameters may contribute to the inability to validate the $3 \mathrm{D}$ models or obtain $3 \mathrm{D}$ models of higher resolution.

Despite the failed tilt validation, the correctness of all 3D models very likely, as datasets of distinct cohesin complexes resulted independently in very similar 3D models. As there are no inconsistencies regarding the proposed assignment of Pds5, SA1, Smc1 $/ 3^{\text {bonsai }}$, the 3D models are reasoned to be authentic. The fact that a Pds5 - bonsai cohesin tetramer dataset, which was shown to contain Pds5-free complexes, was used to obtain and improve the 3D model of the bonsai cohesin tetramer, strongly supports the validity of the 3D models. Moreover, the gold standard validation approach processed by Jan Kaesler generated a 3D model similar to the previously obtained 3D models (Figure 3.13). Further improvement of the similarity are likely, as Jan Kaesler could not obtain a final 3D model and did not sort and divide the dataset according to distinct composition or conformation. In particular, the definition of a sorting strategy is long-winded as, by definition, no prior knowledge is allowed to be applied for an ab initio reconstruction.

As shown previously, sample heterogeneity is still limiting the in case of Pds5 - bonsai cohesin tetramer and in case of the other bonsai cohesin complexes (Figure 3.8a). Head segment rotations, wobbling of the supposed SA1 subunit and flexible interconnecting arms (the proposed coiled-coils) were detected. To large extends, this heterogeneity can be explained by cohesin-specific features. First of all, naturally, cohesin is very flexible due to its coiled-coils, and even bonsai cohesin may allow, due to the remaining coiled-coil fragments, a certain amount of intrinsic flexibility. Even more, the high proportion of HEAT and/or ARM repeats (Pds5, Wapl and SA1) may result in a flexible nature of cohesin. As this heterogeneity seems to result from overall movements instead of locally restricted 
movements, it will be difficult to overcome this heterogeneity computationally. Improved stabilising conditions during sample preparation and further biochemical strategies may be needed to allow higher resolved 3D models. Specifically, the remaining flexibility of the bonsai coiled-coils may be problematic, as they connect the main regions $\left(\mathrm{Smc} 1 / 3^{\text {bonsai }}\right.$ hinge domain with Smc1/3 $\left.3^{\text {bonsai }} \mathrm{NBDs}\right)$. The Wapl $\cdot$ Pds5 $\cdot$ bonsai cohesin tetramer dataset was prepared with a GraFix-treated sample at reduced temperature $\left(-10^{\circ} \mathrm{C}\right)$ to possibly decrease the dynamics of the complex. Moreover, grids of a Pds5 - bonsai cohesin tetramer sample, which was prepared equivalently at reduced temperature $\left(-10^{\circ} \mathrm{C}\right)$, are ready for image recording and data processing. The potentially positive effect of sample preparation at reduced temperature can only be evaluated by comparison of final results with those of an equivalent dataset prepared at standard temperature.

Up to now, image processing of negative stain cohesin data performed better than image processing of vitrified cohesin data. Successful image alignment of cryo datasets, especially without reference 3D model, is more difficult due to the lower contrast compared to negative stain data. In the case of small particle size, particle heterogeneity and pseudosymmetry, the low contrast can be very limiting. So far, the main limitations seem to be related to the heterogeneity. Accordingly, the sample and sample preparation need to be optimised to increase the achievable resolution. Thereafter, it needs to be tested if the data of vitrified sample can be used more successfully.

\subsubsection{Integration of Available High-Resolution Models}

To relate the defined 3D models with the available crystal structures of cohesin (Haering et al., 2004; Kurze et al., 2011; Hara et al., 2014; Gligoris et al., 2014), the crystal structures were fitted into the EM maps. For representation, 3D model t3 is chosen. Initially, the available crystal structures were transformed into density maps and down-filtered to $20 \AA$. Thereby, the information content of all fitted 3D models is equivalent and comparable to the low-resolution EM model. The down-filtered X-ray models were manually pre-fitted and then auto-fitted for final positioning into the EM map.

All down-filtered X-ray models can be fitted adequately and without overlay into 3D model t3, while all corresponding EM densities are filled (Figure 4.2). Only for Pds5 no X-ray structure is available. Thus, the extracted Pds5 density of 3D model t2 is included. The successful fitting, which is in accordance to the subunit definition solely by comparing different EM structures (t1-t6), is a strong indication for the correctness of the fitting. With the EM 3D model and the X-ray data, it is possible to identify each subunit within the corresponding EM density. The absolute handedness of the EM 3D models has not been defined yet. This is only possible by successful tilt strategies or higher resolved 
models. However, the proposed fitting makes the illustrated handedness of the 3D model likely, as mirrored models could not be fitted as good.

\subsubsection{Intermolecular and Intramolecular Crosslinking on Bosai Cohesin}

Chemical cross-linking of complexes in combination with mass spectrometry (MS) allows to identify protein-protein interaction interfaces and protein folding interfaces (reviewed in Walzthoeni et al. (2013)). With cross-linkers, specific reactive groups can be targeted within the complex, while the length of the linker defines the distance of target groups to be cross-linked. Proteolytic digestion and subsequent MS analysis enables the identification of proximity between the targeted reactive groups. The technique of cross-linking MS has improved since the early 2000s, while the analysis of more complex biological samples, like protein complexes, is still tedious. By Dr. Pim Huis in 't Veld, bonsai cohesin was purified and cross-linked with disuccinimidyl suberate (DSS). The sample was analysed by Dr. Franz Herzog by mass spectrometry. Mainly, lysines which are at most 20-25 apart are cross-linked. Details of the procedure can be reviewed elsewhere (Huis in 't Veld, 2013).

A proximity map of interactions between the different subunits was successfully generated and illustrated by Dr. Pim Huis in't Veld (Figure 4.3). In the case of bonsai cohesin, many interactions between Smc1/3 $3^{\text {bonsai }}$ are formed, while specifically Smc1 $/ 3^{\text {bonsai }}$ NBDs seem to be in proximity. The N-terminus of Scc1 appears to interact mainly with Smc3, but not with Smc1. This is in accordance with the structurally defined asymmetry of Scc1 interaction. Only few cross-links between Smc1 $/ 3^{\text {bonsai }}$ and Pds5B were identified, while mainly Smc1 $/ 3^{\text {bonsai }}$ NBDs seem to interact with Pds5b. This is in strong agreement with the structurally determined location of Pds5, which has a curved appearance and is positioned at the bottom between Smc1/3 $3^{\text {bonsai }} \mathrm{NBDs}$ in $3 \mathrm{D}$ model t2. The middle section of Scc1 interacts strongly with SA1, while no interactions between Scc1 and Pds5 was identified. Interestingly, Dr. Pim Huis in't Veld showed biochemically that Scc1 is essential for Pds5 interaction in bonsai cohesin (Huis in 't Veld, 2013). However, the MS approach does not reveal all possible lysine proximities, as the accessibility of certain lysines can be reduced.

A detailed analysis of the MS data and the shown 3D models is limited by the low resolution of the obtained 3D models and the low number of cross-links. Therefore, it is not yet possible to evaluate the accuracy of structural fitting, especially as the X-ray structures mostly lack the proximal lysines. Most convincing is the accordance of Pds5, Smc1 $1^{\text {bonsai }}$ NBD and Smc3 ${ }^{\text {bonsai }}$ NBD proximities between EM and MS data. The positioning of 
a

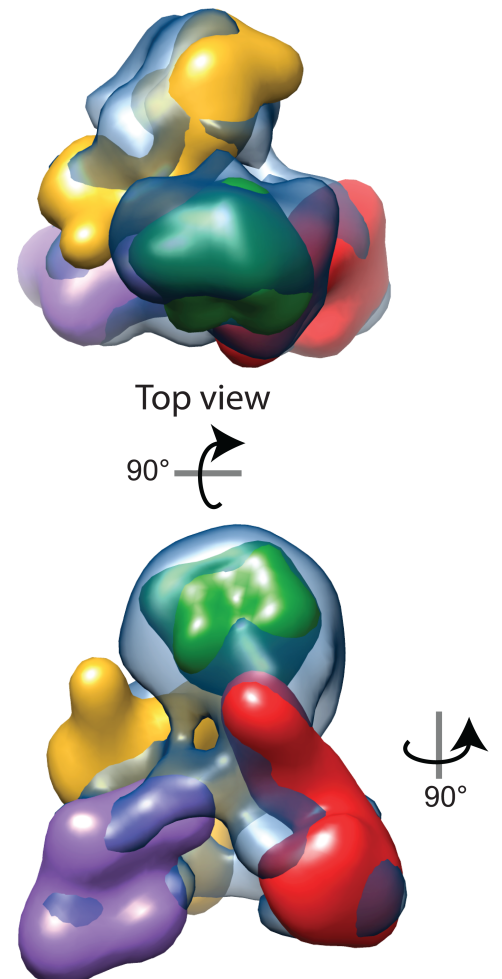

Front view

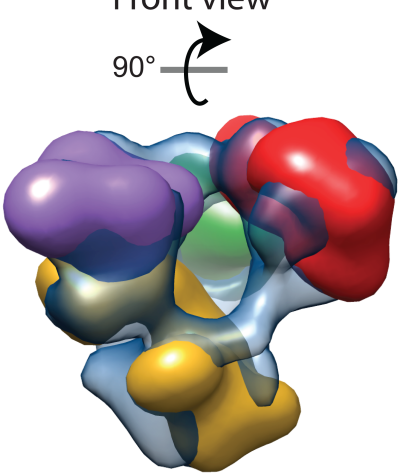

Bottom view
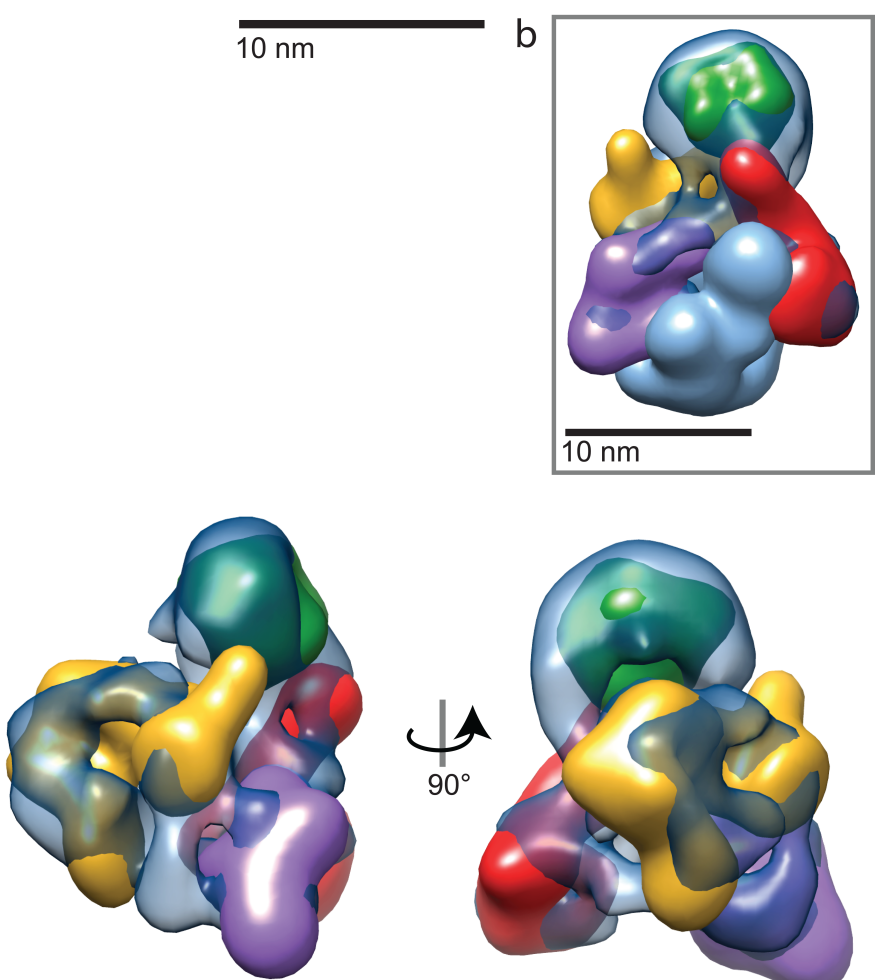

Lateral view (left)

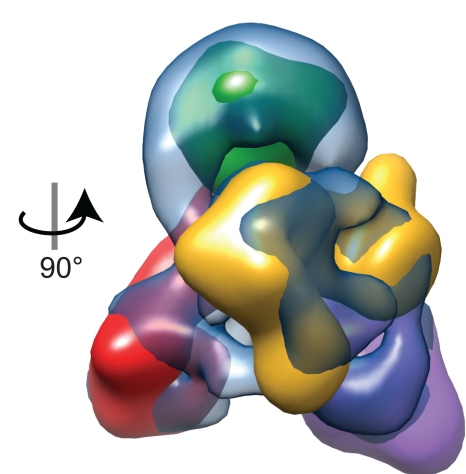

Back view

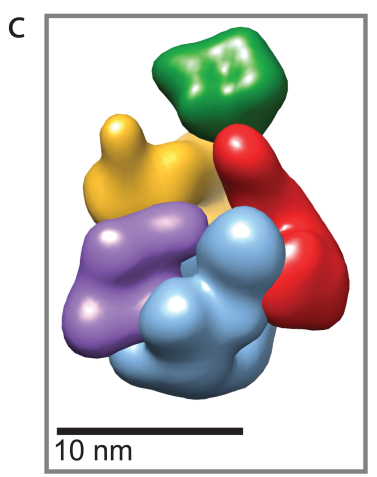

Figure 4.2: Integration of Structural Data. a: A transparent version (blue) of 3D model t3, which was shown to correspond to Pds5-free bonsai cohesin tetramer, is illustrated. Moreover, the previously described 3D structures (Figure1.6) are down-filtered and fitted into the corresponding densities (Haering et al., 2004; Kurze et al., 2011; Hara et al., 2014; Gligoris et al., 2014). The four previously defined segments were filled completely by the down-filtered X-ray structures. Especially, the Smc1/3 NBDs (purple and red) were fitted properly. Interestingly, the C-terminal Scc1 fragment bound to Smc1 NBD (purple) was fitted precisely into the position, where the 2xRFP tag was shown to be located (Figure 3.11). This can be understood as a strong indication for a correct Smc $1 / 3^{\text {bonsai }}$ assignment. The coiled-coil fragments of Smc1/3 NBD crystal structures point to the proposed hinge domain. In Figure3.7e, the central cavity in the crystal structure of the hinge domain was shown to overlay with the cavity in the donut-shaped head fragment. The down-filtered hinge domain (green) is fitted here equivalently. SA1 fills the clasp, as can be seen in the back view. b: The isolated Pds5 density (from 3D model t2) can be fitted properly at the bottom close to the Smc1/3 $3^{\text {bonsai }}$ NBD. c: The illustration in b is shown without $3 \mathrm{D}$ model t3. 
Pds5 at the bottom of Smc1/3 $3^{\text {bonsai }}$ NBDs is in agreement with the identified interactions between Pds5 and Smc1/3 $3^{\text {bonsai }}$ around their NBDs (Figure 4.3). The many interactions of Scc1 and SA1 as analysed by MS may explain the difficulties to distinguish the SA1 density from parts of Scc1. As shown in the 3D model t5 of the Pds5 - bonsai cohesin trimer, a density which could not be assigned so far remains at the backside ("finger"). This may correspond to parts of Scc1, Pds5 or both. In summary, the MS data is in accordance to the obtained 3D models and subunit assignment, specifically for Pds5, although a more detailed comparison of MS and EM data is not possible until higher resolved 3D models are obtained. The compositional and conformational heterogeneity of the complex may imply that there is a blurring in the cross-linking data as well.

a

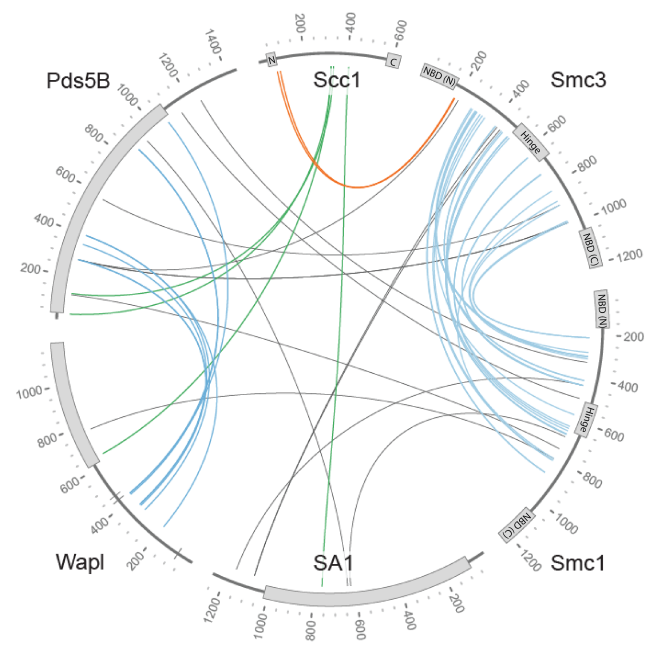

full-length cohesin

intermolecular

cross-links b

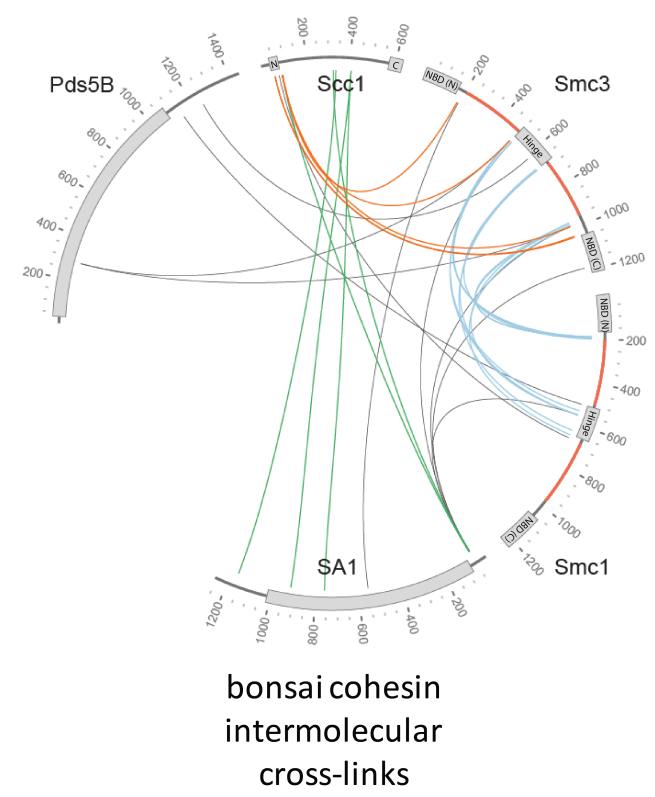

Figure 4.3: Cross-links within Full-Length Cohesin and Bonsai Cohesin. Dr. Pim Huis in't Veld and Dr. Franz Herzog (Gene Center Munich) collaborated to perform chemical cross-linking of recombinant cohesin followed by mass spectrometry analysis. Details of the sample preparation and the obtained data can be reviewed elsewhere (Huis in 't Veld, 2013). Here, the identified cross-links are shown using Circos (Krzywinski et al., 2009) as illustrated by Dr. Pim Huis in't Veld. a: The identified full-length cohesin intermolecular cross-links are depicted. Many contacts could be shown between Smc1 and Smc3 coiled-coils. Moreover, many interactions between Wapl and Pds5B are identified. Contacts between Scc1 and Pds5B were shown as well. b: In the bonsai cohesin complex, the number of identified interactions between Smc1 $1^{\text {bonsai }}$ and $\mathrm{Smc} 3^{\text {bonsai }}$ is reduced (compare full-length cohesin in a). However, in proximity to the Smc1/3 $3^{\text {bonsai }} \mathrm{NBD}$, many interactions are identified. The middle fragment of Scc1 shows strong interaction to SA1, which is in accordance to the crystal structure of Scc1 (middle)-SA2. Moreover, the N-terminus of Scc1 shows many contacts to $\mathrm{Smc} 3^{\text {bonsai }}$, relating to the crystal structure of Scc1(N)-Smc3 ${ }^{\text {bonsai }}$ (Figure1.6). The EM data agrees with the identified interactions between Pds5 and Smc1 $13^{\text {bonsai }}$ close to their NBDs. 


\subsubsection{Model Building of Pds5 · Full-Length Cohesin}

Although, the bonsai design may have an influence on the overall structure it is assumed that the shortened coil-coils do not affect the interaction of Pds5 and SA1 with the Smc1/3 NBDs. On the basis of the structural results on bonsai cohesin, a model of Pds5 - full-length cohesin is proposed, where Pds5 is interacting with both Smc1/3 NBDs. The N-terminus of Scc1 is known to interact with the Smc3 coiled-coil in proximity to the Smc3 NBD, the middle part of Scc1 interacts with SA1 and the C-terminus of Scc1 interacts with Smc1 NBD (1.6). SA1 seems to bridge both Smcs most likely at the Smc1 NBD side, as the Smc1/3 hinge domain is, due to the $50 \mathrm{~nm}$ coiled coils, far apart. It is not known if there is a direct structural interaction of the hinge domain with the globular domain by back-folding. 


\subsection{Influence of Viomycin and Polyamines on 70S Ribosome Dynamics}

A considerable effect of viomycin on the 30S body dynamics was shown, where viomycin stabilises the rotated state. Thus, the results of this work contradict the shown non-rotated state in the X-ray structure (Stanley et al., 2010) and confirm the suggestions based on FRET measurements (Ermolenko et al., 2007; Cornish et al., 2008). Moreover, different $\mathrm{Mg}^{2+}$ concentrations do not alter the influence of viomycin. The different results of previous studies can be explained partly by the different applied approaches. The nonrotated state in the crystal structure of viomycin-bound 70S ribosome may be explained by the crystal packing that can have a strong influence on the dynamic landscape of the 70S ribosome and may cover the influence of viomycin. The induced rotation may be suppressed. In general, the dynamics are likely to be reduced in a crystal and may not represent the naturally occurring dynamics. Single molecule FRET experiments allow studies of molecular dynamics. However, only specific changes in distance between integrated FRET pairs can be analysed. Accordingly, the overall dynamics may remain hidden. In this study, by cryo EM, the first labelling-independent confirmation that viomycin induces a rotated state of the 30S body is given. It remains open to which extend stabilised rotated state contribute to the inhibitory action of viomycin.

Moreover, the effects of polyamines have been tested. In this work, it is shown that, by addition of high polyamine concentrations ( $8 \mathrm{mM}$ putrecine and $1 \mathrm{mM}$ spermidine), a rotated state can be induced. The amount of rotated particles is lower than in the case of viomycin, but the direction of the effect is similar. This observation raises the question if polyamines can modify translocation rates by influencing the dynamics of the $30 \mathrm{~S}$ body. However, an inhibitory effect of polyamines is unlikely as polyamines are present at relatively high concentration in many cells. The reason for the effect of polyamines is not known, but it is likely that it is related to the amount of positive charge and their chain length. Accordingly, their effect may be based on the action as unspecific molecular clamp. The precise effect of polyamines may be an interesting topic for further studies.

Revealing the influence of viomyin on the tRNA dynamics is challening due to the substantial heterogeneity. Regarding the size of the 70S ribosome of $2.5 \mathrm{MDa}$, tRNAs are very small $(26 \mathrm{kDa})$. Sorting for one tRNA correlates to the sorting of $\sim 1 \%$ of the ribosomal mass. Regarding the strong dynamics and the similarity between different states (e.g pre1 and pre2), sorting data on this level is very difficult. In this work, results were obtained by 3D MSA and a reference-based approach. By 3D MSA, it seems that most tRNAs are in an A/P \& P/E (pre5) state. Some tRNAs appear to be in an A/A $\& \mathrm{P} / \mathrm{E}$ (pre3 or pre4) state. Moreover, -/- \& P/E states were observed (empty A-site). 
Interestingly, more ribosomes with -/- \& $\mathrm{P} / \mathrm{E}$ states, were observed in fraction $1\left(2.5^{\circ}\right)$ than in fraction $2\left(7.5^{\circ}\right)$. Previous measurements and data related to this work confirmed that, ribosomes at rotation angles close to the $0^{\circ}$ maximum contain none or only one bound (P-site) tRNA (data not shown). However, in general the tRNAs are blurry and refinement did not result in improved tRNA densities. By using a reference-based approach, sorting resulted in a plot shown in Figure 3.17c. Most tRNAs were attributed to pre3 to pre5 (tRNA hybrid) state and a lower fraction of tRNAs seemed to be in pre1 (classical) state. However, the refinement resulted in 3D models with tRNAs of still limited quality. Although the ratio between pre3 and pre4 relative to pre5 varies between the 3D MSA result and the reference-based approach, they agree that most of the tRNAs seem to be in a hybrid state like in earlier studies (Pan et al., 2007; Ermolenko et al., 2007; Cornish et al., 2008). An improved sorting needs to be applied to increase the quality of the tRNA appearance. Then, in a comparative approach, tRNA states can be compared between viomycin-bound and viomycin-free pre-TC. So far, the data seems to support the stabilisation of a tRNA hybrid state when viomycin is bound. Using 3D classification in RELION with vacant $70 \mathrm{~S}$ ribosome as references ( $0^{\circ} 30 \mathrm{~S}$ body rotation), it is attempted to improve tRNA sorting. Preliminary results appear promising as the tRNA appearance improved. By several iterations of sorting, it is aimed to split the dataset into different tRNAs states. 


\section{Chapter 5}

\section{Summary and Outlook}

Nature composes some of her loveliest poems for the microscope and the telescope.

— Theodore Roszak

\subsection{Topology of an engineered human cohesin com- plex}

Prior to this work, crystal structure models of different subunits of cohesin had been solved and had provided detailed insights into the function of these respective subunits. These structures include the Smc1/Smc3 hinge domain, a SA2-Scc1 complex and a Smc1 NBD-Scc1(C) complex. Very recently, a Smc3 NBD-Scc1(N) complex had been solved and characterised as the DNA exit gate. In figure 1.6 these structures are illustrated in comparison to a complete cohesin model. However, the overall structure was unknown and a topological arrangement of these subunits was not possible.

For this purpose, a bonsai cohesin with shortened coiled-coils was engineered and used for single particle electron microscopy. In this work, the first 3D model revealing the topology of cohesin is shown. The most detailed 3D model (t4) reaches a resolution of roughly $27 \AA$. Accordingly, in this low-resolution map, a reliable fitting of the mentioned crystal structures is hardly possible. Still, by comparing independently obtained 3D models of different subcomplexes (bonsai cohesin tetramer, Pds5 · bonsai cohesin tetramer, Pds5 · bonsai cohesin trimer), it was possible to assign the crystal structures to specific EM densities, despite the limited resolution in the EM 3D map. A prominent and elongated EM density was attributed to Pds5. No crystal structure is available for Pds5 and the binding site of Pds5 had been unknown, previously. Accordingly, this is the first cohesin 3D model including the regulative subunit Pds5. In this work, Pds5 was shown to be located at the bottom of Smc1/3 NBDs bending around both Smc1/3 NBDs. Even more, by comparing Pds5 - bonsai cohesin tetramer with Pds5 - bonsai cohesin trimer, a density corresponding to SA1 is proposed. Pds5 seems to touch this density on the backside of cohesin. The 
crystal structure of the Smc1/3 hinge domain was fitted into a donut-shaped density (head segment) at the top of the 3D model. Due to the similarity between Smc1 and Smc3, a tag was introduced to distinguish Smc1 NBD from Smc3 NBD. An additional density is proposed to correspond to this tag, and thereby a successful distinction between Smc1 NBD and Smc3 NBD appears likely.

The limited resolution may result partly from the fact that all generated 3D models suffer from heterogeneity. Partly, this heterogeneity was reduced by computational sorting. Thereby, the compositional heterogeneity regarding the Pds5 occupancy was be defined, as Pds5-bound and Pds5-unbound 3D models were reconstructed from the same dataset. Still, a large amount of overall conformational heterogeneity is assumed and was not solved by sorting. This heterogeneity can be observed at different positions, for example in the proposed SA1, the coiled-coils and the hinge region.

Small, heterogeneous and pseudo-symmetric objects like bonsai cohesin are structurally challenging. It is aimed to improve the $3 \mathrm{D}$ models of cohesin to obtain higher resolution information. To achieve this, the sample preparation may be optimised further. GraFix was performed at $-10^{\circ} \mathrm{C}$ and a dataset was generated. However, it was not yet quantitatively tested if this preparation decreased the heterogeneity. Further modifications in the GraFix protocol, higher concentrations of $\mathrm{ATP} \gamma \mathrm{S}$ to stabilise the Smc1/3 NBDs, or specific crosslinkers may be tested to address the heterogeneity during sample preparation. Moreover, improved sorting strategies during image processing may be possible. For example, the use of normal mode analysis (NMA) may help to simulate flexibility and sort the dataset for the simulated conformational heterogeneity. Additionally, the potential of the prepared cryo datasets of bonsai cohesin tetramer and Pds5 - bonsai cohesin tetramer has not been exhausted yet. Improved 3D models from negative stain data can be used as reference.

Nevertheless, even at low resolution, many interesting question are still addressable. Most important to mention is the localisation of the Wapl density in a bonsai cohesin complex. Wapl is known to interact with Pds5, but it is not known where exactly this interaction takes place. A dataset of Wapl · Pds5 - bonsai cohesin tetramer was recorded and initial calculations were performed. The initial 3D models are promising, and it is likely that Wapl can be localised. Besides, it is expected that the current 3D model of Pds5 - bonsai cohesin trimer (t5) can be further improved with a bigger dataset. Data sorting was not performed at a similar afford on this dataset as in the case of Pds5 - bonsai cohesin tetramer.

As outlined previously, the validation of a new 3D model is important but difficult in the case of cohesin. These difficulties are reasoned to be equivalent to those limiting high-resolution 3D models so far, namely the small size, the heterogeneity and the pseudosymmetry. The gold standard approach to validate the Pds5 - bonsai cohesin tetramer 
3D model (t2) showed that a similar 3D model with a different dataset can be obtained. However, it is expected that this similarity can be further increased as the 3D model has not stabilised yet and no image sorting has been performed.

\subsection{Viomycin and Polyamine Effects on 70S Ribo- some Dynamics}

Prior to this work, the effect of viomycin on the the 70S ribosome had remained contradictory. While the binding site of viomycin had been defined in the vincinity of h44 (30S subunit) and H69 (50S subunit), and a stabilisation of the A-site tRNA had been shown, the effect of viomycin on the ribosome dynamics had been unknown. Still, it had appeared likely that the inhibitory action on translocation is related to an impact on ribosome dynamics. Therefore, the effects of viomycin on 30S body rotation and on tRNA dynamics were studied in this work. With respect to the tRNA dynamics, it was aimed to analyse an effect of viomycin on classical state or hybrid state stabilisation.

In this work, it was shown that viomycin changes the distribution of the $30 \mathrm{~S}$ body rotation. With viomycin, higher rotations are favoured, as the $7.5^{\circ}$ body rotation peak is increased, while the $0^{\circ}$ body rotation peak is decreased. Moreover, this effect is independent of the used $\mathrm{MgCl}_{2}$ concentration. Additionally, high concentrations of polyamines (spermidine and putrescine) can induce a similar effect on $30 \mathrm{~S}$ body rotation, albeit the effect is smaller. Interestingly, an increased 30S body rotation induced by one viomycin molecule can be mimicked by the collaborative action of several polyamine molecules. It is known that higher degrees of $30 \mathrm{~S}$ body rotation are loosely coupled to more frequent hybrid states. Accordingly, the effect of viomycin on tRNA dynamics was tested. Preliminary results suggest a favoured hybrid state when viomycin is present, possibly similar to the pre4 state. However, the sorting on tRNA levels did not generate high-resolution tRNA densities. The tRNA remained bulky, so that it is expected that the sorting procedure can be improved. 3D MSA and supervised classification had been tested. Supervised classification generated better defined tRNA densities than 3D MSA did.

While the effect of viomycin on 30S rotation was clearly shown, the effect on tRNA level is more difficult to address. Although intermediate results are generated, which seem to relate to a hybrid state when viomycin is added, it is expected that the sorting strategies can be improved. Moreover, the same sorting strategy needs to be performed with a negative control sample without viomycin. So far, it was focused on the optimisation of the sorting strategy with a viomycin-added sample rather than on a preliminary comparative result (+/- viomycin) on tRNA level. An unsupervised maximum-likelihood approach (RELION) had been tested and may result in an improved sorting on tRNA level. When 
this is achieved, a comparative analysis on the effect of viomycin on tRNA level is possible. This result can prove that studying the influence of a small molecule on macromolecular complex dynamics by structural means like single particle cryo EM is an appropriate way to address the inhibitory effect of the small molecule very specifically. In this context, the inhibition can be understood as an altered dynamical landscape. This procedure can be transferred onto other inhibited systems, to study the dynamical aspect of inhibition. 


\section{Appendix A}

\section{Appendix Figures}

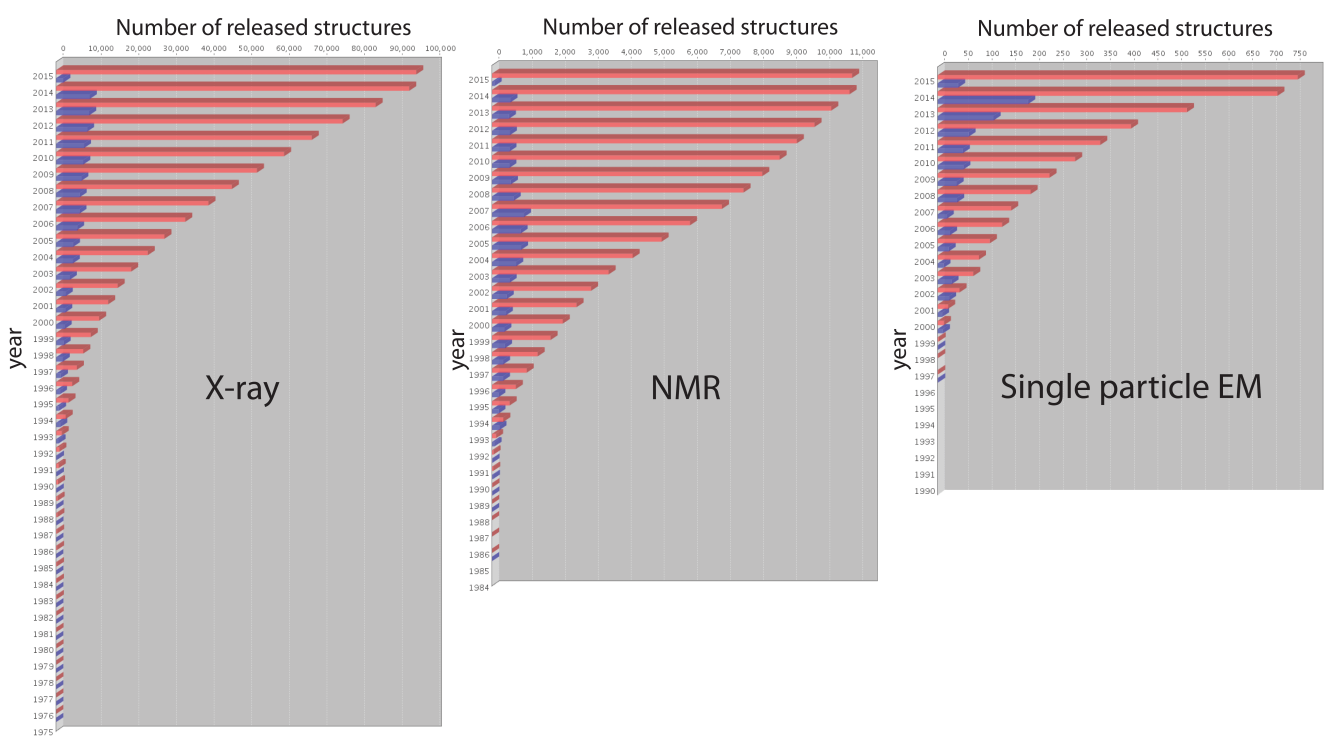

Figure A.1: Number of Released Structures by Different Structural Methods. A comparison between X-ray, NMR and single particle electron microscopy is given. 


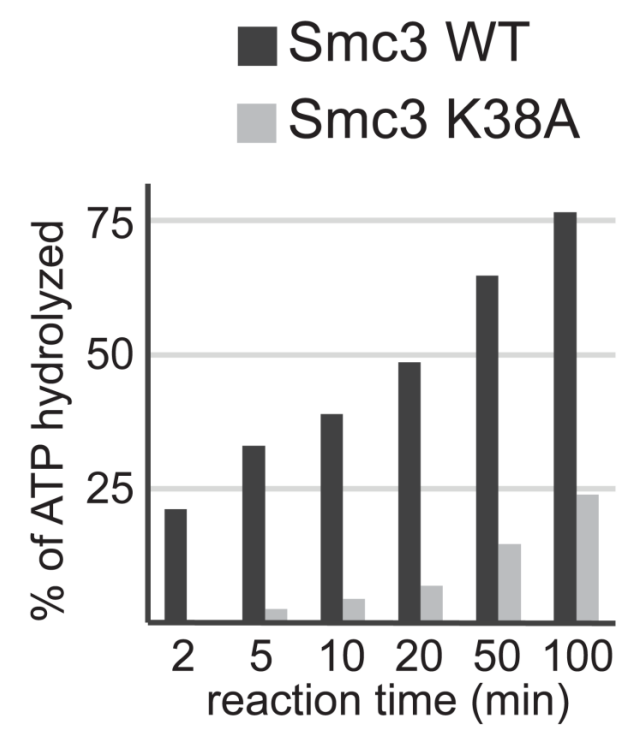

Figure A.2: ATPase Activity of Bonsai Smc3. In an ATPase hydrolysis assay, bonsai Smc3 shows a stronger activity than the K28A mutant (an ATPase insufficiency mutant). Bonsai Smc3 can form a functional ATP binding and hydrolysis pocket. The activity of full-length cohesin and bonsai cohesin appears comparable (data not shown.). 


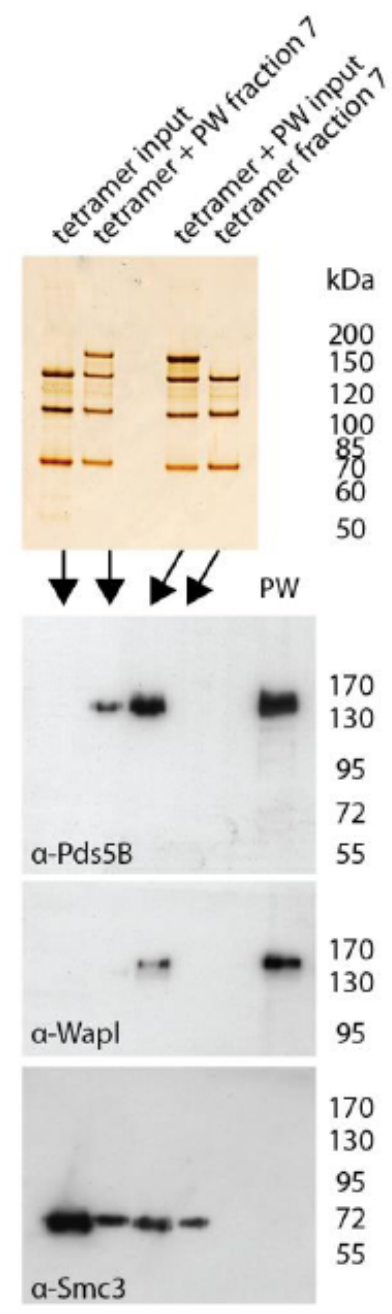

Figure A.3: Substoichiometric Wapl Contamination. Presence of Wapl in different Complexes in comparison to Pds5B and Smc3. 


\section{EM density (t2) Homology model}
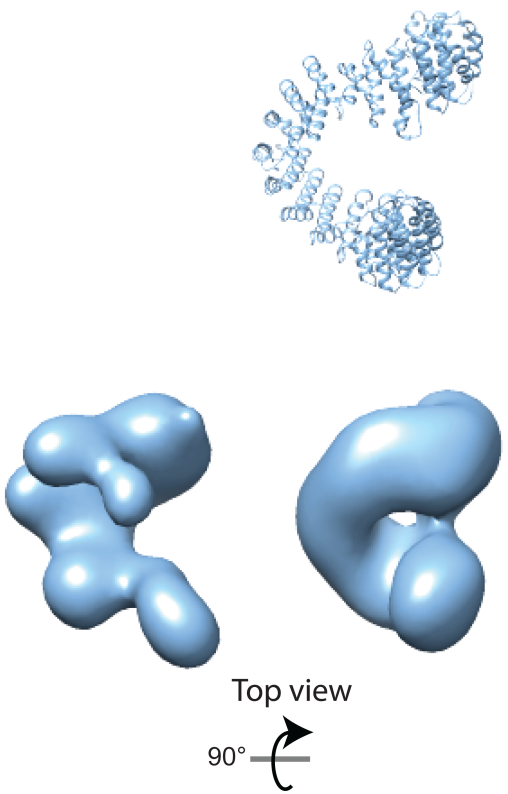

EM density (t2) Homology model

EM density (t2) Homology model
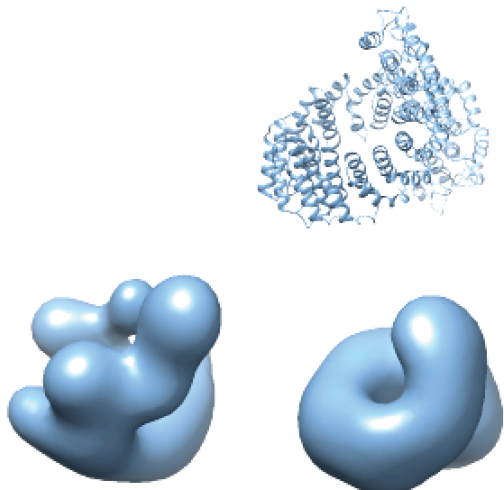

Front view
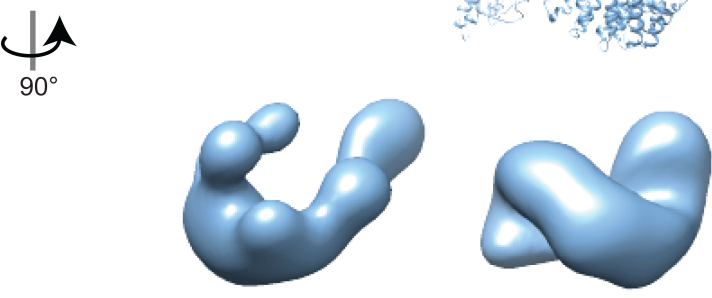

Lateral view (left)

Figure A.4: Comparison of the Extracted Pds5 Density and a Homology Model. The extracted EM density (from 3D model t2), the homology model and a down-filtered version of the homology model is shown in Top view, Front view and Lateral view (left). The homology model was prepared with HHpred (Soding, 2005). 


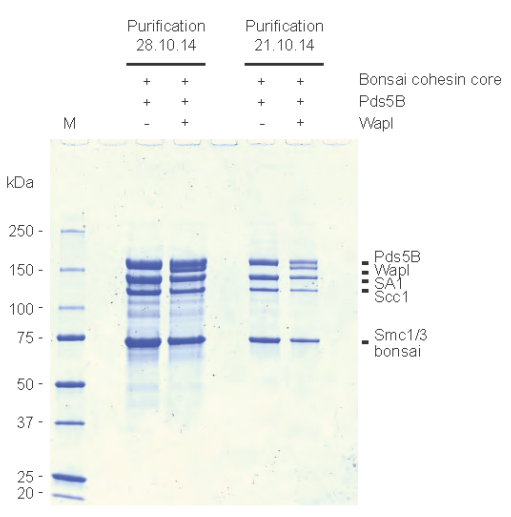

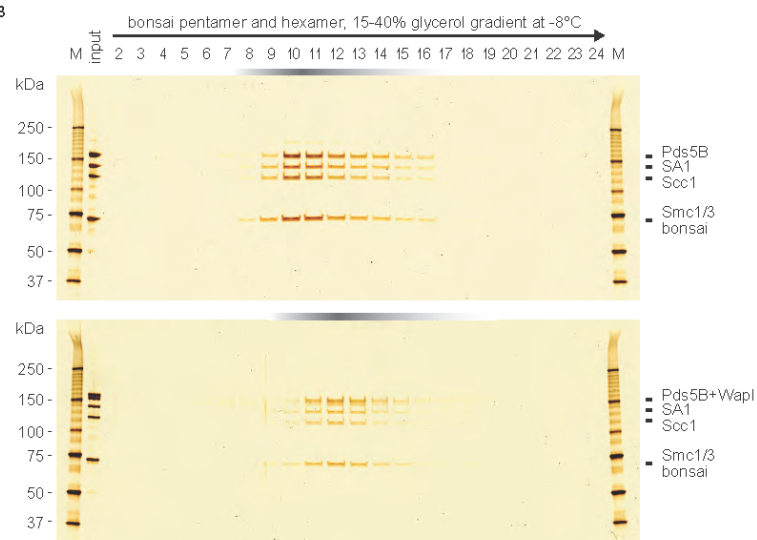

negative staining EM after gradient with stabilizing crosslinker $(80 \%)$
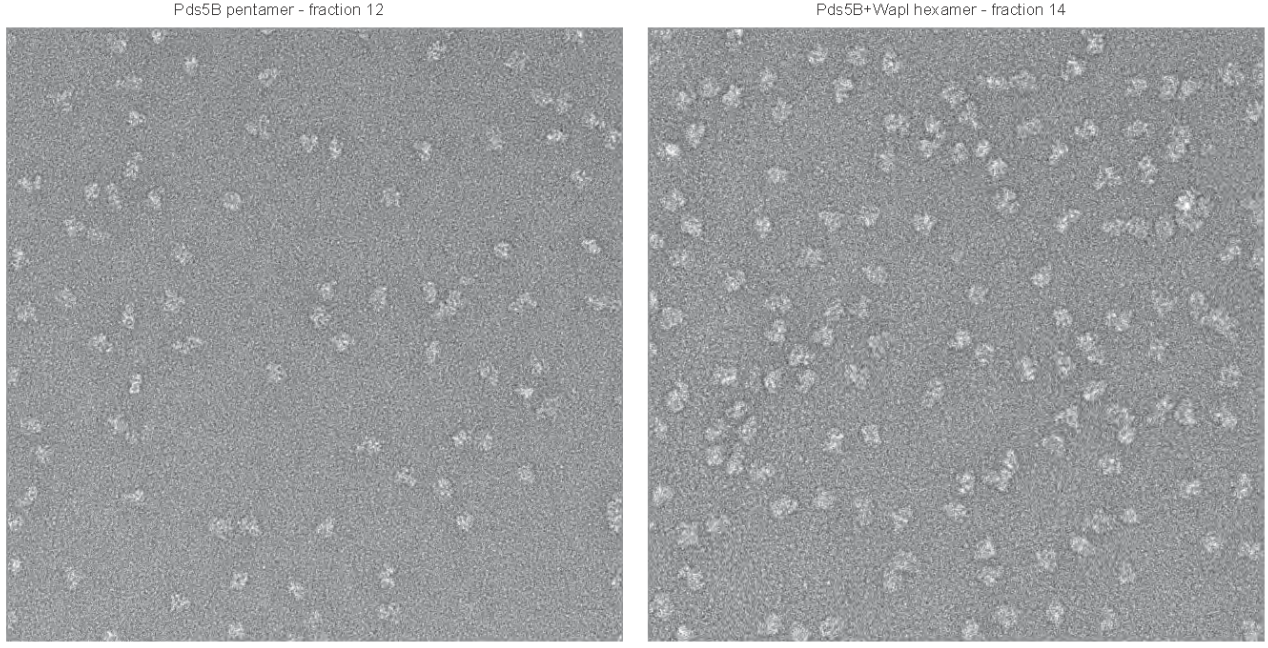

Figure A.5: Sample Quality of Wapl • Pds5 - Bonsai Cohesin Tetramer. A: In SDS-PAGE the binding of Wapl was verified. B: A shift of the peak fraction was obtained for the Wapl · Pds5 - bonsai cohesin tetramer in comparison to Pds5 - bonsai cohesin tetramer. C: After negative stain preparation particles of satisfying sample quality were obtained. 
a

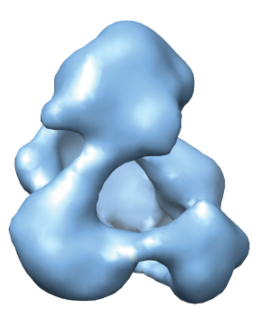

b

$$
\underbrace{}_{180^{\circ}}
$$

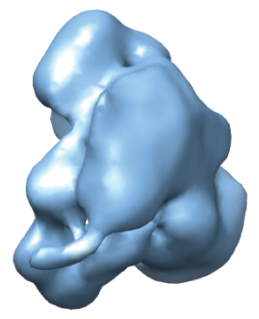

Model1 of Wapl · PDS5 - bonsai Cohesin Tetramer angles obtained by angular reconstitution
Front views

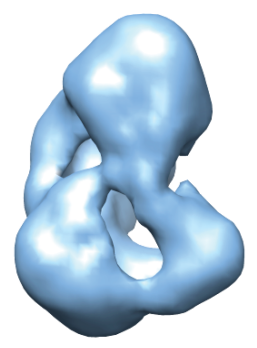

$$
\underbrace{}_{180^{\circ}}
$$

Back views

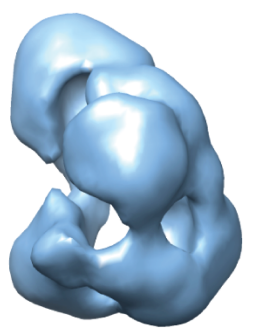

Model2 of Wapl · PDS5 - bonsai Cohesin Tetramer angles obtained by projection matching

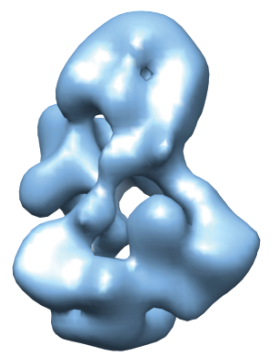

$\underbrace{}_{180^{\circ}}$

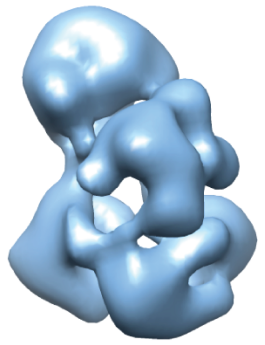

Model t 2 of PDS5 - bonsai Cohesin Tetramer for comparison

Figure A.6: Initial 3D models of Wapl - Pds5 - Bonsai Cohesin Tetramer. a: The front views of two initial 3D models (left, middle) of a Wapl · Pds5 · bonsai cohesin tetramer dataset are shown in comparison to 3D model t2 (right). The left 3D model was generated by angular reconstitution of selected class averages, while the middle 3D model was obtained by taking the angular information from most similar (highest CCC) projection images of a used Pds5 - bonsai cohesin tetramer reference. b: The respective back views are depicted. Both 3D models are of limited quality and do not allow any conclusions. Still, a similarity to 3D model t2 can be observed and further image processing and sorting is expected to generate a 3D model, where the Wapl location can be assigned. The 3D models were obtained by Jan Kaesler under supervision of the author of this work. 
a

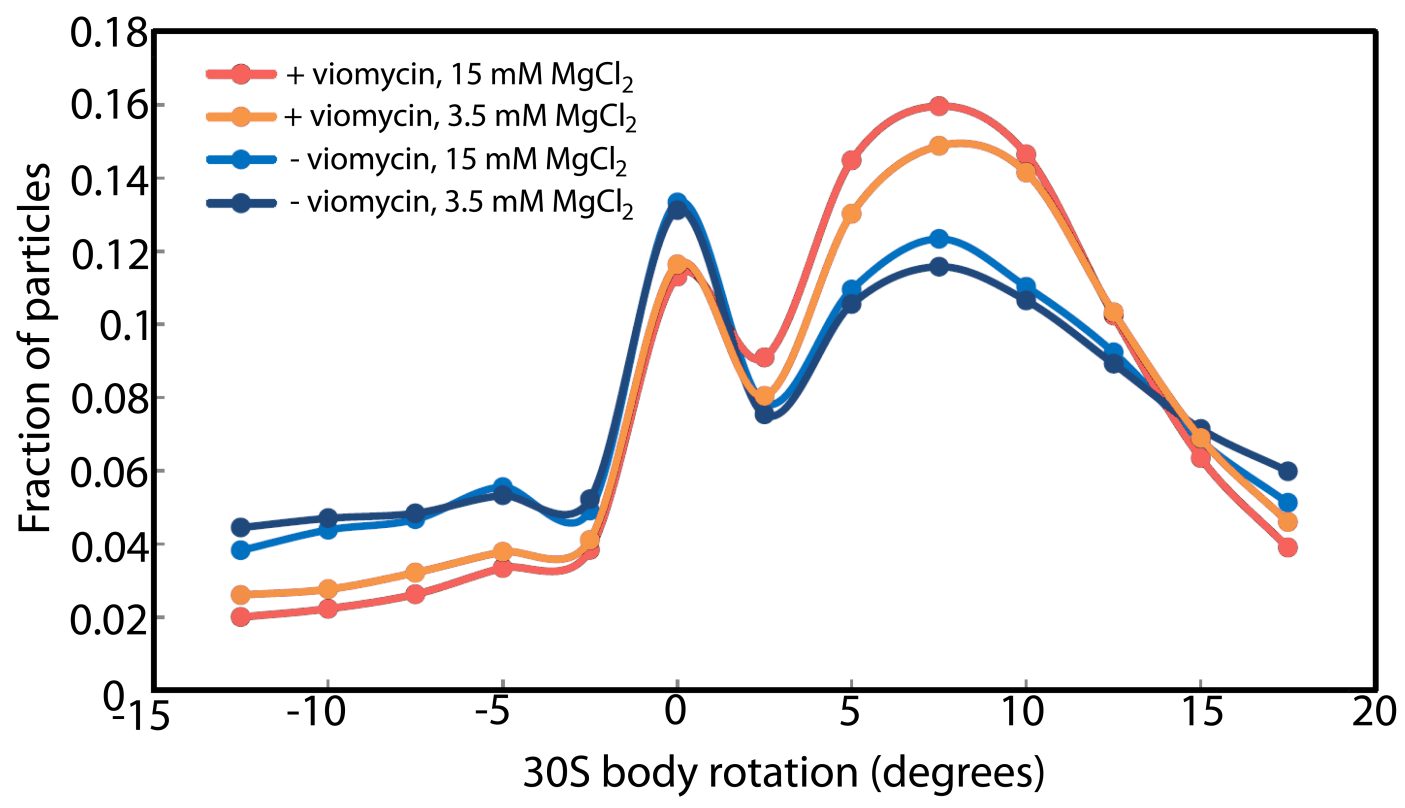

Figure A.7: Viomycin Effect on 30S Body Rotation. Comparison of +/- viomycin condition on 30S body rotation for all tested buffers. 


\section{Appendix B}

\section{Appendix Text}

\section{B.1 Crystallography and X-Ray Diffraction}

X-ray diffraction is the oldest structural method and dates back to 1912, when Max von Laue and others discovered diffraction of X-rays in crystals. Motivated by this work, Bragg senior and junior discovered that X-rays can be used to detect atomic coordinates within crystals ${ }^{1}$. The following basic informations about X-ray diffraction are summarised according to Lottspeich and Engels (2012).

The physical basis of diffraction is the interaction of the X-ray with the electron shell of an atom emitting a secondary X-ray due to coherent and elastic scattering ${ }^{2}$. The primary beam interferes stationary with this secondary beam, generating local regions of constructive and destructive interference. According to Bragg's law, the direction of these reflexes relates to the crystal lattice and can be used to define the crystal unit cell. The overall intensity of the reflections correlates with the size of the crystal and the regularity of the crystal lattice. Mathematically, the relation between the atomic position in the unit cell and the diffracted X-ray beam is a Fourier transformation. Each beam carries amplitude, wavelength and phase information. During detection the phase information is lost. Therefore, there are several methods which define the phase information indirectly, by using either known similar phase information ("molecular replacement") or extracting the phase from intensity changes of native and heavy metal salt-soaked crystals ("isomorphic replacement"). Both techniques make use of the Patterson function. Basically, the electron density can be calculated from the structure factor amplitude, which is proportional to the square root of the intensity (I) of a reflection spot. Further ways to solve the phase problem are based on "multi-wavelength anomalous diffraction" (MAD) and "single-wavelength anomalous diffraction" (SAD). In both cases, anomalous diffraction of bound heavy metal atoms is based on the influence of inner electrons.

\footnotetext{
${ }^{1}$ Consequently, 2014 was defined as the international year of crystallography, exactly 100 years after the Physics Nobel Prize for Max von Laue.

${ }^{2}$ This is a simplification, as in reality there is inelastic scattering, resulting in higher wavelength X-rays, but their contribution is very low $(25 \mathrm{meV}$ energy loss compared to an energy of $10 \mathrm{keV})$.
} 
One major limiting step in X-ray structure analysis is the expensive and time-consuming procedure of generating crystals, as high quality crystals (0.1-0.3 mm edge length) are needed. Crystallisation conditions are searched in automated buffer screens, making use of sitting or hanging drop methods. Usually, several mg of protein are needed to define crystal forming conditions. Once good conditions are identified, the crystal is mounted in a thin liquid film with a nylon loop and measured under nitrogen cooling with an $\mathrm{X}$-ray beam. The X-ray beam is generated either by a rotating anode tube or by the more intense and wavelength-adaptable synchrotron source.

It becomes very challenging to grow crystals of larger assemblies (e.g. of macromolecular complexes), especially if they are very heterogeneous in conformation (flexibility) and composition (regulatory factors). Besides, crystal may be insufficient due to lattice defects. The definition of the asymmetric unit and the phase can become very sophisticated for very large assemblies. This is the reason for the upper size limit for the accessible molecular weight of an analyte. Only rare exceptions (e.g the 80S ribosome (Ben-Shem et al., 2011), the mammalian fatty acid synthase (Maier et al., 2008), the 20S proteasome (Groll et al., 1997), the RNA polymerase II (Cramer et al., 2001) have been solved so far, while others (e.g. the spliceosome, the nuclear-pore-complex and many transmembrane proteins) stay out of reach. Using micro crystals in free-electron lasers is an interesting development in this field. Usually, it is not possible to evaluate dynamics, as usually in crystals all entities are in the same conformation, and defining crystallisation conditions for a different conformation is tedious. The quality of an electron density map can be evaluated by the declared resolution, the $\mathrm{R}$ value, the $\mathrm{R}$-free value and the $\mathrm{B}$-factor.

\section{B.2 Nuclear Magnetic Resonance Spectroscopy}

The phenomenon of "Nuclear Magnetic Resonance" (NMR) was detected in 1945. Here, it will be briefly summarised according to Lottspeich and Engels (2012). Using a homogeneous magnetic field, the energy level of the nuclear spin of a proton can be manipulated and different energy levels will be generated. Using a resonant electromagnetic wave, the energy difference can be compensated and measured by a change of the electromagnetic induction. The physical basis is the magnetic moment of an atomic nucleon in an external magnetic field. Usually, nuclei with similar numbers of protons and neutrons neutralise their antipodal quantum mechanic spin. Thus, only those nuclei with an uneven number of protons and neutrons generate a magnetic spin unequal to zero. Accordingly, ${ }^{1} \mathrm{H}$ is of main interest for NMR. Moreover, the signal of ${ }^{13} \mathrm{C},{ }^{15} \mathrm{~N}$ or ${ }^{31} \mathrm{P}$ is often used, especially as those isotopes can be artificially introduced into the molecule of interest to increase the signal. Each nuclear spin can have several orientations, depending on the isotopic spin 
quantum number $(\mathrm{I})$, while there can be $2 I+1$ orientations. Accordingly, a proton can have two orientations with similar energy. Only due to an alternating magnetic field, their energy level will differ ("Zeeman effect"). This energy difference is proportional to the strength of the external magnetic field and the nucleus-specific gyromagnetic ratio. To compensate for this energy difference, a resonant electromagnetic wave, usually in the range of radio waves, can be used. The corresponding frequency is the "Larmor frequency". Today, radio wave pulses are used, which have a specific frequency spectrum, resulting in the simultaneous excitation of all magnetic spins. Very strong and constant magnetic fields are generated with superconducting electromagnets. The individual Larmor frequency of each magnetic spin can be extracted from the sum of these signals by using "fast Fourier transformation" (FFT). Depending on the chemical environment of each proton or ${ }^{13} \mathrm{C}$ atom, the Larmor frequency will alternate (leading to "chemical shift"), making it possible to extract structural information from these frequencies. Therefore, the chemical composition can be extracted from the frequency of the signal, whereas the integral of the signal is proportional to the relative number of corresponding nuclei. Close nuclei with a spin $\neq 0$ modulate each other's signal ("scalar coupling"). The measured signal is the "free induction decay" (FID), which is represented after FFT as an NMR spectrum. Compared to other methods, the signal strength of NMR is relatively low due to low-energy differences and it is increased by strengthening the magnetic field, lowering the temperature, or including more nuclei. With increasing size, the spectrum of an analyte becomes very complicated as multidimensional NMR spectra are used, where similar ("homo nuclear COSY") or different nuclei ("heteronuclear COSY") are correlated. This is done by scalar coupling or the "nuclear Overhauser effect" (NOE). Usually, protein concentrations in the range of $0.1-10 \mathrm{mM}$ are needed. The required sample amount is smaller than in X-ray crystallography but higher than in cryo EM (Table 1.1). As nucleus proximity and chemical environment are measured directly, NMR can access a the complete range of molecular dynamics, depending only on the interpretability of the spectra. Moreover, this interpretability sets an upper limit for accessible analytes, so that particles of up to $100 \mathrm{kDa}$ can rarely be processed. Outstanding examples of NMR on macromolecular complexes are shown for the dihydrofolate reductase (Boehr et al., 2006) and E. coli ribosome Christodoulou et al. (2004).

\section{B.3 Mass Spectrometry}

"Mass spectrometry" (MS) has developed very quickly from an analytical method for revealing chemical composition of low-molecular substances to a very powerful biochemical technique important for protein sequencing, biochemical analysis (e.g. posttranslational 
modifications) and structural biology ${ }^{3}$ (Walzthoeni et al., 2013). The theory of MS will be briefly summarised according to Lottspeich and Engels (2012). In MS analytes are sorted based on the mass-to-charge ratio $(m / Q)$. Thus, a mass spectrometer is composed of an ionisation source, a mass analyser, where the sorting based on $m / Q$ occurs, and a detector that counts the collision events. Although there are several other ionisation methods, the main ways to generate ions from proteins are "electrospray ionisation" (ESI) and "matrix-assisted laser desorption" (MALDI). To generate an electrospray, a high electric field is applied to a small capillary containing the sample in solution. The droplets of the generated aerosol travel into the direction of the electric field. As the solvent evaporates, the charge density becomes higher, until the droplets explode to nanometer size ("Rayleigh limit"). The charge accumulates on the analyte and it is possible to generate a stream of single-charged analytes. In MALDI, the analyte is mixed with 1000- to 10000-fold excess of organic matrix molecules that absorb light in the UV spectrum. After co-crystallisation, a UV-laser pulse is emitted onto the sample, which explodes into gas phase due to excitation. Both, analyte and matrix molecules, are ionised and travel along the electric field. Both methods allow a mild ionisation of macromolecular complexes.

There are several different mass analyser systems, like the "time of flight" (TOF) analyser and the quadrupole. TOF can be used to define the mass-charge ratio. As the acceleration voltage is known and the time of flight after acceleration ("drift distance") to the detector can be measured very precisely, the ratio of molecular mass and charge can be defined. The time of flight is proportional to $(m / Q)^{2}$. Thus, the molecular mass can be defined by the time of flight. Differently, a quadrupole allows only ions of certain m/Q ratio to oscillate on a stable path, while all others are stopped. This is typically used with ESI since derived ions have lower energy than in a MALDI approach. Similarly, an ion trap can be used to collect and release certain ions, which can be utilised in combination with MALDI as well. Detection of ions usually works with an electron amplifier. By separating a complex into the corresponding subunits under denaturing conditions, the accurate mass of each subunit can be defined. The stoichiometry can be obtained by acquiring MS data of the intact complex. Tandem MS analysis allows fragmentation of the complex in order to distinguish between core and peripheral subunits. Finally, chemical cross-linking MS allows the generation of a protein-protein interaction network, with information of proximity, binding site and possible oligomeric states. So, the generated information provides topological information but no atomistic model. Using homology models, EM or X-ray data and "small-angle X-ray scattering" (SAXS) data, this method allows structural modelling in a hybrid approach. In many cases, one method alone does

\footnotetext{
${ }^{3}$ Nature methods chose mass spectrometry-based proteomics as "method of the year" in 2012. In 2002, the Chemistry Nobel Prize was awarded for soft desorption ionisation methods for mass spectrometry, specifically the development of "electron spray ionisation" (ESI) and "matrix-assisted laser desorption/ionisation" (MALDI).
} 
not fulfil the requirements to understand the biology of a macromolecular complex. MS has shown to be of very high significance to tackle these obstacles. Very nice examples of mass spectrometric contribution to structural questions are shown in the cases of the RNA polymerase I (Jennebach et al., 2012) and RNA polymerase II-TFIIF (Chen et al., 2010b), the TRiC/CCT chaperonin (Leitner et al., 2012), the protein phosphatase 2A (Herzog et al., 2012) and the 26S proteasome (Lasker et al., 2012b).

\section{B.4 Methods to Adress Biomolecular Dynamics}

In the following section, some approaches will be summarised according to Lottspeich and Engels (2012). One very powerful method is single-molecule FRET (smFRET), which is based on the radiationless energy transfer between a donor and an acceptor fluorophore in proximity, while the signal strength decreases proportional to the 6th power of the Förster radius $\left(r^{6}\right)$. Usually, total internal reflection fluorescence (TIRF) or confocal microscopy are used to excite only fluorophores in a very defined imaging layer. Several FRET pairs, introduced via site-specific labelling techniques, can be used in parallel, allowing detection of several distance trajectories over time. Since spatial and temporal resolution in FRET assays are very high, site-specific dynamics of known structures can be analysed in great detail. Still, this technique permits only selective information of the dynamics of a labelled position, whereas global dynamics are not feasible.

Finally, there are methods using a priori available structural data. If an atomic model is available, molecular dynamics (MD) (Karplus and Mccammon, 2002) and normal mode analysis (NMA) (Lindahl et al., 2006; Nogales-Cadenas et al., 2013) can be applied. The first MD simulation of a protein was published in 1977 for bovine pancreatic trypsin inhibitor (BPTI) (McCammon et al., 1977). Since then, computational power and software were improved dramatically, e.g. by parallel computing. In MD simulations, the motion of macromolecules of roughly up to one million atoms can be described at an atomistic scale. As the solution of the time-dependent Schrödinger equation is even for single hydrogen atoms not possible, several approximations are made for a feasible calculation time. In principle, independent electron movements are ignored and directly coupled to much slower nuclei movements. Still, these effective nuclei potentials are simplified by introducing force fields, which are mathematically easier to describe. Finally, Newton's second law is used to describe atomic motions, which hence can be solved numerically. Periodic boundary conditions allow the calculations in the computed box to be free of boundary effects. Energy minimisation, a prerequisite for MD simulation, is accomplished by relaxation of the structure of interest into adequate solvent interactions. For many systems, isothermal and isobaric conditions are important, which are simulated by introducing thermostats and 
barostats. By MD simulation, movements and solvent interactions of many biomolecules have been predicted in accordance to experimental results (Hagen et al., 1995; Rasmussen et al., 1992). For many aspects related to slow dynamics, NMA is better suited and less CPU-demanding (Skjaerven et al., 2009). In NMA the approximation is made that only the lowest frequencies of motion (soft modes) are sufficient to describe the main movements of the analysed object. Higher frequencies can be ignored. For this purpose, the motion can be decomposed in reciprocal space by multivariate statistical analysis. Using only the lower frequency domain, defined by the linear combination of the main eigenvectors, a sufficient model of the protein's flexibility can be generated. NMA is used very successfully for atomistic coordinate maps of PDB entries. For EM models pseudo-atomistic structures can be generated by a coarse-grain-representation of the EM densities. These pseudo-atomistic structures provide a good approximation for modelling the large-scale conformational changes, but need to be validated by other means. Many web-based NMA tools are available for PDB structures, while NOMAD-Ref and 3DEM Loupe can be used for EM densities (Lindahl et al., 2006; Nogales-Cadenas et al., 2013). 


\section{Appendix C}

\section{Abbreviations}

1D one-dimensional

2D two-dimensional

3D three-dimensional

aa amino acid

ABC ATP binding cassette

AFM atomic force microscope

ADP adenosine di phosphate

AMPPCP 5'-adenylyl ( $\beta, \gamma$-methylene)diphosphonate

APC/C anaphase promoting complex /cyclosome

ARM armadillo repeat

ASL anticodon stem-loop

ATP adenosine tri phosphate

$\operatorname{ATP}_{\gamma} \mathbf{S}$ adenosine 5-[ $\gamma$-thio $]$ triphosphate

BSA buried surface area

BPTI bovine pancreatic trypsin inhibitor

BSE back scattered electrons

CCC cross correlation coefficient

CCD charge coupled device

CCF cross correlation function

CDK cyclin-dependent kinases

CMOS complementory metal oxide semiconductors

COSY correlation spectroscopy 
CPU central processing unit

CRM1 chromosome region maintenance factor 1

cryo EM electron cryo-microscopy

CTF contrast transfer function

DDD direct detection device

DFT discrete Fourier transformation

DMSO dimethyl sulfoxide

DNA desoxy-ribonucleic acid

DQE detector quantum efficiency

DSB double-strand break

DSS disuccinimidyl suberate

EF-G elongation factor $G$

EF-Tu elongation factor thermo unstable

EM electron microscopy

EMDB Electron Microscopy Data Bank

EPR electron paramagnetic resonance

ER endoplasmatic reticulum

ESI electrospray ionisation

FEG field emission gun

FEL free-electron laser

FFT fast Fourier transform

FID free induction decay

fMet N-formylmethionine

FRAP fluorescence recovery after photobleaching

FRC Fourier ring correlation

FRET Foerster resonance energy transfer

FSC Fourier shell correlation

G gap 
GA glutaraldehyde

GPU graphical processing unit

GraFix gradient fixation

GTP guanosine tri phospate

h44 helix 44 (30S subunit)

H69 Helix 69 (50S subunit)

H89 Helix 89 (50S subunit)

H91 Helix 91 (50S subunit)

HAKM1 HEPES, ammonium chloride, $\mathrm{K}$ for $\mathrm{KCl}$ and magnesium chloride

HEAT huntingtin, elongation factor 3, protein phosphatase 2A and TOR1

HEPES 4-(2-hydroxyethyl)-1-piperazineethanesulfonic acid

HF high frequency

HPD hybrid pixel detectors

IC initiation complex

IF $\mathbf{1 / 2 / 3}$ initiation factor $1 / 2 / 3$

$\mathbf{K}_{d}$ dissociation constant

L 1/5/12/16/27 large 1/5/12/16/27 (protein 50S subunit)

LF low frequency

M mitosis

MALDI matrix-assisted laser desorption/ionisation

MAPS monolith active pixel sensors

MD molecular dynamics

ML maximum likelihood

MRA multi-reference alignment

mRNA messenger ribonucleic acid

MS mass spectrometry

MSA multivariate statistical analysis

MTF modulation transfer function 
NBD nucleotide binding domain

NDC80 nuclear division cycle 80

NHD N-terminal helical domain

Ni-NTA nickel-nitrilotriacetic acid

NMA normal mode analysis

NMR nuclear magnetic resonance

NOE nuclear Overhauser effect

NRPS non-ribosomal peptide synthetases

PCA principal component analysis

PDB Protein Data Bank

PCTF phase contrast transfer function

Pds5 precocious dissociation of sisters protein 5

PEP phosphoenolpyruvate

Phe phenylalanin

PIC pre-initiation complex

PK pyruvate kinase

PMPI N-(p-Maleimidophenyl)isocyanate

PP2A protein phosphatase $2 \mathrm{~A}$

Pt/C carbon-supported Pt nanoparticles

pre-RC pre-replicative complex

PSF point spread function

PTC peptidyl transferase center

pre-TC pre-translocation complex

RCT random conical tilt

$\mathbf{R F} \mathbf{1 / 2} / \mathbf{3}$ release factor $1 / 2 / 3$

RFP red fluorescent protein

RNA ribonucleic acid

RNP ribonucleoprotein 
$\mathbf{R R F}$ ribosome release factor

S synthesis

S7 small 7 (protein 30S subunit)

SA1 stromal antigen 1

SAD single-wavelength anomalous diffraction

SAXS small-angle X-ray scattering

Sc1/2 scaffold $1 / 2$

Scc1 Sertoli cell intracellular protein 1

SDS-PAGE sodium dodecyl sulfate-poly acryl amide gel electrophoresis

SE secondary electrons

SEM scanning electron microscope

Sgo1 shugosin

Smc structural maintenance of chromosomes

sm-FRET single molecule Förster resonance energy transfer

SNR signal-to-noise-ratio

SRL sarcin-ricin loop

SSNR spectral signal-to-noise-ratio

STAG3 stromal antigen 3

TC ternary complex

TEM transmission electron microscope

TFIIF transcription factor II F

TIRF total internal reflection fluorescence

TOF time-of-flight

TPPP tilt-pair parameter plot

TRiC/CCT TCP-1 ring complex/chaperonin containing TCP-1

tRNA transfer ribonucleic acid

UV ultraviolet

WHD winged-helix domain 
YFP yellow fluorescent protein 


\section{Bibliography}

Adolph, K. W., Cheng, S. M., and Laemmli, U. K. (1977). Role of Nonhistone Proteins Chromosome Structure in Metaphase. Cell, 12(November):605-616.

Adrian, M., Dubochet, J., Fuller, S. D., and Harris, J. R. (1998). Cryo-negative staining. Micron, 29(2-3):145-160.

Adrian, M., Dubochet, J., Lepault, J., and McDowall, a. W. (1984). Cryo-electron microscopy of viruses. Nature, 308(5954):32-36.

Al-Amoudi, A., Chang, J.-J. J.-J., Leforestier, A., McDowall, A. W., Salamin, L. M., Norlén, L. P. O., Richter, K., Blanc, N. S., Studer, D., Dubochet, J., M., S. L., Norlen, L. P. O., and Sartori, N. (2004). Cryo-electron microscopy of vitreous sections. EMBO Journal, 23(18):3583-3588.

Alberts, B., Johnson, A., Lewis, J., Raff, M., Roberts, K., and Walter, P. (2008). Alberts Molecular biology of the cell 5th Ed. Garland, New York.

Allan Drummond, D. and Wilke, C. O. (2009). The evolutionary consequences of erroneous protein synthesis. Nat Rev Genet, 10(10):715-724.

Anderson, D. E., Losada, A., Erickson, H. P., and Hirano, T. (2002). Condensin and cohesin display different arm conformations with characteristic hinge angles. The Journal of Cell Biology, 156(3):419-424.

Andrade, M. a., Perez-Iratxeta, C., and Ponting, C. P. (2001a). Protein repeats: structures, functions, and evolution. Journal of structural biology, 134(2-3):117-131.

Andrade, M. a., Petosa, C., O’Donoghue, S. I., Müller, C. W., and Bork, P. (2001b). Comparison of ARM and HEAT protein repeats. Journal of molecular biology, 309(1):118.

Baker, L. A. and Rubinstein, J. L. (2010). Radiation Damage in Electron Cryomicroscopy. In Cryo-EM Part A Sample Preparation and Data Collection, volume Volume 481, pages $371-388$. Academic Press.

Baker, T. S. and Henderson, R. (2012). Electron cryomicroscopy of biological macromolecules. International Tables for Crystallography, F:593-614. 
Ban, N., Freeborn, B., Nissen, P., Penczek, P., Grassucci, R. A., Sweet, R., Frank, J., Moore, P. B., and Steitz, T. A. (1998). A 9 ÅResolution X-Ray Crystallographic Map of the Large Ribosomal Subunit. Cell, 93(7):1105-1115.

Ban, N., Nissen, P., Hansen, J., Moore, P. B., and Steitz, T. a. (2000). The complete atomic structure of the large ribosomal subunit at 2.4 Åresolution. Science, 289(August):905 \pm 920 .

Barkei, J. J., Kevany, B. M., Felnagle, E. A., and Thomas, M. G. (2009). Investigations into Viomycin Biosynthesis Using Heterologous Production in Streptomyces lividans. Chembiochem, 10(2):366-376.

Ben-Shem, A., Garreau de Loubresse, N., Melnikov, S., Jenner, L., Yusupova, G., and Yusupov, M. (2011). The Structure of the Eukaryotic Ribosome at 3.0 ÅResolution. Science, 334(6062):1524-1529.

Beringer, M. and Rodnina, M. V. (2007). The Ribosomal Peptidyl Transferase. Molecular Cell, 26(3):311-321.

Beringer, M., Rodnina, M. V., and Wolfenden, R. (2004). The ribosome as an entropy trap. 101(21):12397-12398.

Blanchard, S. C., Cooperman, B. S., and Wilson, D. N. (2010). Probing Translation with Small-Molecule Inhibitors. Chemistry and Biology, 17(6):633-645.

Blanchard, S. C., Kim, H. D., Gonzalez, R. L., Puglisi, J. D., and Chu, S. (2004). tRNA dynamics on the ribosome during translation. Proceedings of the National Academy of Sciences of the United States of America, 101(35):12893-12898.

Bock, L. V., Blau, C., Schröder, G. F., Davydov, I. I., Fischer, N., Stark, H., Rodnina, M. V., Vaiana, A. C., and Grubmüller, H. (2013). Energy barriers and driving forces in tRNA translocation through the ribosome. Nature structural $\&$ molecular biology, 20(12):1390-6.

Boehr, D. D., McElheny, D., Dyson, H. J., and Wright, P. E. (2006). The dynamic energy landscape of dihydrofolate reductase catalysis. Science, 313(5793):1638-1642.

Boehr, D. D., Nussinov, R., and Wright, P. E. (2009). The role of dynamic conformational ensembles in biomolecular recognition. Nat Chem Biol., 5(11):789-796.

Borland, L. and van Heel, M. (1990). Classification of image data in conjugate representation spaces. Journal of the Optical Society of America A, 7(4):601-610.

Boyer, P. D. (1997). The ATP Synthase - a splendid molecular machine. Annual Review of Biochemistry, 66:717-749. 
Bracewell, R. N. (1956). Strip Integration in Radio Astronomy. Australian Journal of Physics, vol. 9, p.198.

Brenner, S. and Horne, R. W. (1959). A negative staining method for high resolution electron microscopy of viruses. Biochimica et biophysica acta, 34:103-110.

Buonomo, S. B., Clyne, R. K., Fuchs, J., Loidl, J., Uhlmann, F., and Nasmyth, K. (2000). Disjunction of homologous chromosomes in meiosis I depends on proteolytic cleavage of the meiotic cohesin Rec8 by separin. Cell, 103(3):387-398.

Bürmann, F., Shin, H.-C., Basquin, J., Soh, Y.-M., Giménez-Oya, V., Kim, Y.-G., Oh, B.H., and Gruber, S. (2013). An asymmetric SMC-kleisin bridge in prokaryotic condensin. Nature structural \&f molecular biology, 20(3):371-9.

Busche, B. (2013). New Algorithms for Automated Processing of Electronmicroscopic Images.

Campbell, M. G., Veesler, D., Cheng, A., Potter, C. S., and Carragher, B. (2015). 2.8 Åresolution reconstruction of the Thermoplasma acidophilum 20S proteasome using cryo-electron microscopy. eLife, 4:1-10.

Carter, a. P., Clemons, W. M., Brodersen, D. E., Morgan-Warren, R. J., Wimberly, B. T., and Ramakrishnan, V. (2000). Functional insights from the structure of the 30S ribosomal subunit and its interactions with antibiotics. Nature, 407(6802):340-348.

Chan, K.-L., Roig, M. B., Hu, B., Beckouet, F., Metson, J., and Nasmyth, K. (2012). Cohesin's DNA exit gate is distinct from its entrance gate and is regulated by acetylation. Cell, 150(5):961-974.

Chebotareva, N. a., Kurganov, B. I., and Livanova, N. B. (2004). Biochemical effects of molecular crowding. Biochemistry, 69(11):1239-1251.

Chen, J., Sawyer, N., and Regan, L. (2013). Protein-protein interactions: General trends in the relationship between binding affinity and interfacial buried surface area. Protein Science, 22(4):510-515.

Chen, J. Z. and Grigorieff, N. (2007). SIGNATURE: A single-particle selection system for molecular electron microscopy. Journal of Structural Biology, 157(1):168-173.

Chen, Y., Koripella, R. K., Sanyal, S., and Selmer, M. (2010a). Staphylococcus aureus elongation factor $\mathrm{G}$ - Structure and analysis of a target for fusidic acid. FEBS Journal, 277(18):3789-3803.

Chen, Z. A., Jawhari, A., Fischer, L., Buchen, C., Tahir, S., Kamenski, T., Rasmussen, M., Lariviere, L., Bukowski-Wills, J.-C., Nilges, M., Cramer, P., and Rappsilber, J. (2010b). 
Architecture of the RNA polymerase II-TFIIF complex revealed by cross-linking and mass spectrometry. EMBO Journal, 29(4):717-726.

Christodoulou, J., Larsson, G., Fucini, P., Connell, S. R., Pertinhez, T. a., Hanson, C. L., Redfield, C., Nierhaus, K. H., Robinson, C. V., Schleucher, J., and Dobson, C. M. (2004). Heteronuclear NMR investigations of dynamic regions of intact Escherichia coli ribosomes. Proceedings of the National Academy of Sciences of the United States of America, 101(30):10949-10954.

Ciferri, C., Pasqualato, S., Screpanti, E., Varetti, G., Santaguida, S., Dos Reis, G., Maiolica, A., Polka, J., De Luca, J. G., De Wulf, P., Salek, M., Rappsilber, J., Moores, C. a., Salmon, E. D., and Musacchio, A. (2008). Implications for kinetochore-microtubule attachment from the structure of an engineered Ndc80 complex. Cell, 133(3):427-39.

Cobbe, N. and Heck, M. M. S. (2004). The Evolution of SMC Proteins: Phylogenetic Analysis and Structural Implications. Molecular Biology and Evolution, 21(2):332-347.

Cornish, P. V., Ermolenko, D. N., Noller, H. F., and Ha, T. (2008). Spontaneous Intersubunit Rotation in Single Ribosomes. Molecular Cell, 30(5):578-588.

Cramer, P., Bushnell, D. a., and Kornberg, R. D. (2001). Structural Basis of Transcription : RNA Polymerase II at 2 . 8 Ångstrom Resolution. 292(June):1863-1876.

Crick, F. H. C., Barnett, L., Brenner, S., and Watts-Tobin, R. J. (1961). General Nature of the Genetic Code for Proteins. Nature, 192(4809):1227-1232.

Csermely, P., Palotai, R., and Nussinov, R. (2010). Induced fit, conformational selection and independent dynamic segments: An extended view of binding events. Trends in Biochemical Sciences, 35(10):539-546.

Cunha, C. E., Belardinelli, R., Peske, F., Holtkamp, W., Wintermeyer, W., and Rodnina, M. V. (2013). Dual use of GTP hydrolysis by elongation factor G on the ribosome. Translation, 1(June):1-11.

Czworkowski, J., Wang, J., Steitz, T. A., and Moore, P. B. (1994). The crystal structure of elongation factor G complexed with GDP, at 2.7 A resolution. EMBO Journal, 13(16):3661-3668.

Daniel E. Koshland (2008). The Seven Pillars of Life. Science, 295(22 March 2002):4-7.

Davidovich, C., Belousoff, M., Wekselman, I., Shapira, T., Krupkin, M., Zimmerman, E., Bashan, A., and Yonath, A. (2010). The proto-ribosome: An ancient nano-machine for peptide bond formation. Israel Journal of Chemistry, 50(1):29-35.

Deardorff, M. A., Bando, M., Nakato, R., Watrin, E., Itoh, T., Minamino, M., Saitoh, K., Komata, M., Katou, Y., Clark, D., Cole, K. E., De Baere, E., Decroos, C., Di Donato, 
N., Ernst, S., Francey, L. J., Gyftodimou, Y., Hirashima, K., Hullings, M., Ishikawa, Y., Jaulin, C., Kaur, M., Kiyono, T., Lombardi, P. M., Magnaghi-Jaulin, L., Mortier, G. R., Nozaki, N., Petersen, M. B., Seimiya, H., Siu, V. M., Suzuki, Y., Takagaki, K., Wilde, J. J., Willems, P. J., Prigent, C., Gillessen-Kaesbach, G., Christianson, D. W., Kaiser, F. J., Jackson, L. G., Hirota, T., Krantz, I. D., and Shirahige, K. (2012). HDAC8 mutations in Cornelia de Lange syndrome affect the cohesin acetylation cycle. Nature, 489(7415):313-317.

Dorner, S., Brunelle, J. L., Sharma, D., and Green, R. (2006). The hybrid state of tRNA binding is an authentic translation elongation intermediate. Nat Struct Mol Biol, 13(3):234-241.

Dreier, M. R., Bekier, M. E. n., and Taylor, W. R. (2011). Regulation of sororin by Cdk1-mediated phosphorylation. Journal of cell science, 124(Pt 17):2976-2987.

Dubochet, J. and McDowall, A. W. (1981). Vitrification of pure water electron microscopy. Journal of Microscopy, 124(3):3-4.

Ellis, R. J. (2001). Macromolecular crowding: An important but neglected aspect of the intracellular environment. Current Opinion in Structural Biology, 11(1):114-119.

Elmlund, D. and Elmlund, H. (2012). SIMPLE: Software for ab initio reconstruction of heterogeneous single-particles. Journal of structural biology, 180(3):420-7.

Elmlund, D. and Elmlund, H. (2015). Cryogenic Electron Microscopy and Single-Particle Analysis. Annual Review of Biochemistry, 84(1):150306093657004.

Elmlund, H., Elmlund, D., and Bengio, S. (2013). PRIME: probabilistic initial 3D model generation for single-particle cryo-electron microscopy. Structure, 21(8):1299-306.

Ericsson, U. B., Hallberg, B. M., DeTitta, G. T., Dekker, N., and Nordlund, P. (2006). Thermofluor-based high-throughput stability optimization of proteins for structural studies. Analytical Biochemistry, 357(2):289-298.

Ermolenko, D. N., Cornish, P. V., Ha, T., and Noller, H. F. (2013). Antibiotics that bind to the A site of the large ribosomal subunit can induce mRNA translocation. $R N A$, $19(2): 158-66$.

Ermolenko, D. N., Spiegel, P. C., Majumdar, Z. K., Hickerson, R. P., Clegg, R. M., and Noller, H. F. (2007). The antibiotic viomycin traps the ribosome in an intermediate state of translocation. Nature structural \& molecular biology, 14(6):493-7.

Faruqi, a. R. and Henderson, R. (2007). Electronic detectors for electron microscopy. Current Opinion in Structural Biology, 17(5):549-555. 
Faruqi, a. R. and Subramaniam, S. (2000). CCD detectors in high-resolution biological electron microscopy. Quarterly reviews of biophysics, 33(1):1-27.

Feldman, M. B., Terry, D. S., Altman, R. B., and Blanchard, S. C. (2010). Aminoglycoside activity observed on single pre-translocation ribosome complexes. Nat Chem Biol, $6(1): 54-62$.

Feynman, R. P. (1964). The Feynman Lectures on Physics.

Fischer, N., Konevega, A. L., Wintermeyer, W., Rodnina, M. V., and Stark, H. (2010). Ribosome dynamics and tRNA movement by time-resolved electron cryomicroscopy. Nature, 466(7304):329-33.

Fischer, N., Neumann, P., Konevega, A. L., Bock, L. V., Ficner, R., Rodnina, M. V., and Stark, H. (2015). Structure of the E. coli ribosome-EF-Tu complex at $<3$ Åresolution by Cs-corrected cryo-EM. Nature.

Frank, J. (1990). Classification of macromolecular assemblies studied as 'single particles'. Quarterly reviews of biophysics, 23(3):281-329.

Frank, J. (2006). Three-Dimensional Electron Microscopy of Macromolecular Assemblies. Oxford University Press, Oxford.

Frank, J. and Agrawal, R. K. (2000). A ratchet-like inter-subunit reorganization of the ribosome during translocation . Nature, 406(6793):318-322.

Frank, J., Shimkin, B., and Dowse, H. (1981). Spider-A modular software system for electron image processing. Ultramicroscopy, 6(4):343-357.

Gao, H., Valle, M., Ehrenberg, M., and Frank, J. (2004). Dynamics of EF-G interaction with the ribosome explored by classification of a heterogeneous cryo-EM dataset. Journal of structural biology, 147(3):283-290.

Gao, Y.-G., Selmer, M., Dunham, C. M., Weixlbaumer, A., Kelley, A. C., and Ramakrishnan, V. (2009). The structure of the ribosome with elongation factor G trapped in the posttranslocational state. Science, 326(5953):694-699.

Gasser, S. M., Laroche, T., Falquet, J., Boy de la Tour, E., and Laemmli, U. K. (1986). Metaphase chromosome structure. Involvement of topoisomerase II. Journal of molecular biology, 188(4):613-629.

Gavin, A.-C., Bösche, M., Krause, R., Grandi, P., Marzioch, M., Bauer, A., Schultz, J., Rick, J. M., Michon, A.-M., Cruciat, C.-M., Remor, M., Höfert, C., Schelder, M., Brajenovic, M., Ruffner, H., Merino, A., Klein, K., Hudak, M., Dickson, D., Rudi, T., Gnau, V., Bauch, A., Bastuck, S., Huhse, B., Leutwein, C., Heurtier, M.-A., Copley, R. R., Edelmann, A., Querfurth, E., Rybin, V., Drewes, G., Raida, M., Bouwmeester, T., Bork, 
P., Seraphin, B., Kuster, B., Neubauer, G., and Superti-Furga, G. (2002). Functional organization of the yeast proteome by systematic analysis of protein complexes. Nature, 415(6868):141-147.

Gerlich, D., Koch, B., Dupeux, F., Peters, J.-M., and Ellenberg, J. (2006). Live-cell imaging reveals a stable cohesin-chromatin interaction after but not before DNA replication. Current biology : CB, 16(15):1571-1578.

Gillespie, P. J. and Hirano, T. (2004). Scc2 couples replication licensing to sister chromatid cohesion in Xenopus egg extracts. Current biology : CB, 14(17):1598-1603.

Glaeser, R. M. and Hall, R. J. (2015). Reaching the Information Limit in Cryo-EM of Biological Macromolecules: Experimental Aspects. Biophysical Journal, 100(10):23312337.

Gligoris, T. G., Scheinost, J. C., Bürmann, F., Petela, N., Chan, K.-L., Uluocak, P., Beckouët, F., Gruber, S., Nasmyth, K., and Löwe, J. (2014). Closing the cohesin ring: Structure and function of its Smc3-kleisin interface. Science, 346(6212):963-967.

Golas, M. M., Sander, B., Will, C. L., Luhrmann, R., and Stark, H. (2003). Molecular architecture of the multiprotein splicing factor SF3b. Science, 300(5621):980-984.

Golden, B. L., Ramakrishnan, V., and White, S. W. (1994). Crystal structure of prokaryotic ribosomal protein 19: a bi-lobed rna-binding protein. EMBO Journal, 12(13):1-8.

Goodsell, D. S. (2009). The Machinery of Life - Second Edition. Springer, New York.

Grigorieff, N. (2007). FREALIGN: High-resolution refinement of single particle structures. Journal of Structural Biology, 157(1):117-125.

Groll, M., Ditzel, L., Löwe, J., Stock, D., Bochtler, M., Bartunik, H. D., and Huber, R. (1997). Structure of $20 \mathrm{~S}$ proteasome from yeast at 2.4 A resolution.

Gruber, S., Arumugam, P., Katou, Y., Kuglitsch, D., Helmhart, W., Shirahige, K., and Nasmyth, K. (2015). Evidence that Loading of Cohesin Onto Chromosomes Involves Opening of Its SMC Hinge. Cell, 127(3):523-537.

Haering, C. H., Farcas, A.-M., Arumugam, P., Metson, J., and Nasmyth, K. (2008). The cohesin ring concatenates sister DNA molecules. Nature, 454(7202):297-301.

Haering, C. H., Löwe, J., Hochwagen, A., and Nasmyth, K. (2002). Molecular Architecture of SMC Proteins and the Yeast Cohesin Complex. Molecular Cell, 9(4):773-788.

Haering, C. H., Schoffnegger, D., Nishino, T., Helmhart, W., Nasmyth, K., and Lo, J. (2004). Structure and Stability of Cohesin's Smc1-Kleisin Interaction. 15:951-964. 
Hagen, S. J., Hofrichter, J., and Eaton, W. A. (1995). Protein reaction kinetics in a room-temperature glass. Science, 269(5226):959-962.

Hagstrom, K. a. and Meyer, B. J. (2003). Condensin and cohesin: more than chromosome compactor and glue. Nature reviews. Genetics, 4(7):520-534.

Haider, M., Uhlemann, S., Schwan, E., Rose, H., and Kabius, B. (1998). Electron microscopy image enhanced. Nature, 392(6678):768-769.

Hansen, J. L., Schmeing, T. M., Moore, P. B., and Steitz, T. A. (2002). Structural insights into peptide bond formation. Proceedings of the National Academy of Sciences of the United States of America, 99(18):11670-11675.

Hara, K., Zheng, G., Qu, Q., Liu, H., Ouyang, Z., Chen, Z., Tomchick, D. R., and Yu, H. (2014). Structure of cohesin subcomplex pinpoints direct shugoshin-Wapl antagonism in centromeric cohesion. Nature Structural Eamp; Molecular Biology, 21(August):1-9.

Harauz, G., Boekema, E., and van Heel, M. (1988). Statistical image analysis of electron micrographs of ribosomal subunits. Methods in enzymology, 164:35-49.

Harauz, G. and van Heel, M. (1986). Exact filters for general geometry three dimensional reconstruction.

Haselbach, D. (2014). Conformational Dynamics of Large Proteins and Protein Complexes. $\mathrm{PhD}$ thesis.

Hauf, S., Roitinger, E., Koch, B., Dittrich, C. M., Mechtler, K., and Peters, J.-M. (2005). Dissociation of cohesin from chromosome arms and loss of arm cohesion during early mitosis depends on phosphorylation of SA2. PLoS biology, 3(3):e69.

Hauf, S., Waizenegger, I. C., and Peters, J. M. (2001). Cohesin cleavage by separase required for anaphase and cytokinesis in human cells. Science, 293(5533):1320-1323.

Havugimana, P. C., Hart, G. T., Nepusz, T., Yang, H., Turinsky, A. L., Li, Z., Wang, P. I., Boutz, D. R., Fong, V., Phanse, S., Babu, M., Craig, S. a., Hu, P., Wan, C., Vlasblom, J., Dar, V. U. N., Bezginov, A., Clark, G. W., Wu, G. C., Wodak, S. J., Tillier, E. R. M., Paccanaro, A., Marcotte, E. M., and Emili, A. (2012). A census of human soluble protein complexes. Cell, 150(5):1068-1081.

Heidinger-Pauli, J. M., Onn, I., and Koshland, D. (2010). Genetic Evidence that the Acetylation of the Smc3p Subunit of Cohesin Modulates Its ATP-Bound State to Promote Cohesion Establishment in Saccharomyces cerevisiae. Genetics, 185(4):12491256. 
Henderson, R. (1995). The potential and limitations of neutrons, electrons and X-rays for atomic resolution microscopy of unstained biological molecules. Quarterly reviews of biophysics, 28(2):171-93.

Henderson, R. (2013). Avoiding the pitfalls of single particle cryo-electron microscopy: Einstein from noise. Proceedings of the National Academy of Sciences of the United States of America, 110(45):18037-41.

Henderson, R., Chen, S., Chen, J. Z., Grigorieff, N., Passmore, L. A., Ciccarelli, L., Rubinstein, J. L., Crowther, R. A., Stewart, P. L., and Rosenthal, P. B. (2011a). Tiltpair analysis of images from a range of different specimens in single-particle electron cryomicroscopy. Journal of molecular biology, 413(5):1028-1046.

Henderson, R., Chen, S., Chen, J. Z., Grigorieff, N., Passmore, L. a., Ciccarelli, L., Rubinstein, J. L., Crowther, R. A., Stewart, P. L., and Rosenthal, P. B. (2011b). Tiltpair analysis of images from a range of different specimens in single-particle electron cryomicroscopy. Journal of Molecular Biology, 413(5):1028-1046.

Henderson, R., Sali, A., Baker, M. L., Carragher, B., Devkota, B., Downing, K. H., Egelman, E. H., Feng, Z., Frank, J., Grigorieff, N., Jiang, W., Ludtke, S. J., Medalia, O., Penczek, P. A., Rosenthal, P. B., Rossmann, M. G., Schmid, M. F., Schröder, G. F., Steven, A. C., Stokes, D. L., Westbrook, J. D., Wriggers, W., Yang, H., Young, J., Berman, H. M., Chiu, W., Kleywegt, G. J., and Lawson, C. L. (2012). Outcome of the first electron microscopy validation task force meeting. Structure (London, England : 1993), 20(2):205-14.

Herzog, F., Kahraman, a., Boehringer, D., Mak, R., Bracher, a., Walzthoeni, T., Leitner, a., Beck, M., Hartl, F.-U., Ban, N., Malmstrom, L., and Aebersold, R. (2012). Structural Probing of a Protein Phosphatase 2A Network by Chemical Cross-Linking and Mass Spectrometry. Science, 337(6100):1348-1352.

Hirano, T., Kobayashi, R., and Hirano, M. (1997). Condensins, chromosome condensation protein complexes containing XCAP-C, XCAP-E and a Xenopus homolog of the Drosophila Barren protein. Cell, 89(4):511-521.

Hirano, T. and Mitchison, T. J. (1994). A heterodimeric coiled-coil protein required for mitotic chromosome condensation in vitro. Cell, 79(3):449-458.

Hirota, T., Gerlich, D., Koch, B., Ellenberg, J., and Peters, J.-M. (2004). Distinct functions of condensin I and II in mitotic chromosome assembly. Journal of cell science, 117(Pt 26):6435-6445. 
Holtkamp, W., Cunha, C. E., Peske, F., Konevega, A. L., Wintermeyer, W., and Rodnina, M. V. (2014). GTP hydrolysis by EF-G synchronizes tRNA movement on small and large ribosomal subunits. EMBO Journal, 33(9):1073-1085.

Hope, H., Frolow, F., von Böhlen, K., Makowski, I., Kratky, C., Halfon, Y., Danz, H., Webster, P., Bartels, K. S., Wittmann, H. G., and Yonath, A. (1989). Cryocrystallography of ribosomal particles. Acta Crystallographica Section B, 45(2):190-199.

Hopfner, K. P., Karcher, a., Shin, D. S., Craig, L., Arthur, L. M., Carney, J. P., and Tainer, J. a. (2000). Structural biology of Rad50 ATPase: ATP-driven conformational control in DNA double-strand break repair and the ABC-ATPase superfamily. Cell, 101(7):789-800.

Horan, L. H. and Noller, H. F. (2007). Intersubunit movement is required for ribosomal translocation. Proceedings of the National Academy of Sciences of the United States of America, 104(12):4881-4885.

Hu, B., Itoh, T., Mishra, A., Katoh, Y., Chan, K.-L., Upcher, W., Godlee, C., Roig, M. B., Shirahige, K., and Nasmyth, K. (2011). ATP hydrolysis is required for relocating cohesin from sites occupied by its Scc2/4 loading complex. Current biology : CB, 21(1):12-24.

Huang, C. E., Milutinovich, M., and Koshland, D. (2005). Rings, bracelet or snaps: fashionable alternatives for Smc complexes. Philosophical transactions of the Royal Society of London. Series B, Biological sciences, 360(1455):537-542.

Huis in 't Veld, P. J. (2013). Reconstitution of the human cohesin complex. PhD thesis.

Huis in 't Veld, P. J., Herzog, F., Ladurner, R., Davidson, I. F., Piric, S., Kreidl, E., Bhaskara, V., Aebersold, R., and Peters, J.-M. (2014). Characterization of a DNA exit gate in the human cohesin ring. Science, 968(November).

Illangasekare, M., Sanchez, G., Nickles, T., and Yarus, M. (1995). Aminoacyl-RNA synthesis catalyzed by an RNA. Science, 267(5198):643-647.

Ivanov, D. and Nasmyth, K. (2005). A topological interaction between cohesin rings and a circular minichromosome. Cell, 122(6):849-860.

Ivanov, D. and Nasmyth, K. (2007). A physical assay for sister chromatid cohesion in vitro. Molecular cell, 27(2):300-310.

Jennebach, S., Herzog, F., Aebersold, R., and Cramer, P. (2012). Crosslinking-MS analysis reveals RNA polymerase I domain architecture and basis of rRNA cleavage. Nucleic Acids Research. 
Jenner, L., Melnikov, S., de Loubresse, N. G., Ben-Shem, A., Iskakova, M., Urzhumtsev, A., Meskauskas, A., Dinman, J., Yusupova, G., and Yusupov, M. (2012). Crystal structure of the 80S yeast ribosome. Current Opinion in Structural Biology, 22(6):759-767.

Johansen, S. K., Maus, C. E., Plikaytis, B. B., and Douthwaite, S. (2006). Capreomycin binds across the ribosomal subunit interface using tlyA-encoded 2'-O-methylations in 16S and 23S rRNAs. Molecular cell, 23(2):173-182.

Joseph, S. and Noller, H. F. (1998). EF-G-catalyzed translocation of anticodon stem-loop analogs of transfer RNA in the ribosome. EMBO Journal, 17(12):3478-3483.

Joyeux, L. and Penczek, P. a. (2002). Efficiency of 2D alignment methods. Ultramicroscopy, 92(2):33-46.

Karplus, M. and Mccammon, J. A. (2002). Molecular dynamics simulations of biomolecules. Nat Struct Biol, 9.

Kastner, B., Fischer, N., Golas, M. M., Sander, B., Dube, P., Boehringer, D., Hartmuth, K., Deckert, J., Hauer, F., Wolf, E., Uchtenhagen, H., Urlaub, H., Herzog, F., Peters, J. M., Poerschke, D., Lührmann, R., and Stark, H. (2008). GraFix: sample preparation for single-particle electron cryomicroscopy. Nature methods, 5(1):53-55.

Kim, H. D., Puglisi, J. D., and Chu, S. (2007). Fluctuations of transfer RNAs between classical and hybrid states. Biophysical journal, 93(10):3575-3582.

Kirves, J.-M. (2014). New Algorithms for Single Particle Cryo Electron Microscopic Image Processing.

Klein, F., Mahr, P., Galova, M., Buonomo, S. B., Michaelis, C., Nairz, K., and Nasmyth, K. (1999). A central role for cohesins in sister chromatid cohesion, formation of axial elements, and recombination during yeast meiosis. Cell, 98(1):91-103.

Kohl, H., Reimer, L., Asakura, T., Kamiya, T., Krausz, F., Monemar, B., Venghaus, H., Weber, H., and Weinfurter, H. (2007). Transmission Electron Microscopy. Springer, New York.

Konevega, A. L., Fischer, N., Semenkov, Y. P., Stark, H., Wintermeyer, W., and Rodnina, M. V. (2007). Spontaneous reverse movement of mRNA-bound tRNA through the ribosome. Nature structural $\&$ molecular biology, 14(4):318-324.

Kong, X., Ball, A. R. J., Pham, H. X., Zeng, W., Chen, H.-Y., Schmiesing, J. A., Kim, J.-S., Berns, M., and Yokomori, K. (2014). Distinct functions of human cohesin-SA1 and cohesin-SA2 in double-strand break repair. Molecular and cellular biology, 34(4):685-698. 
Korostelev, A., Trakhanov, S., Laurberg, M., and Noller, H. F. (2006). Crystal Structure of a 70S Ribosome-tRNA Complex Reveals Functional Interactions and Rearrangements. Cell, 126(6):1065-1077.

Krzywinski, M., Schein, J., Birol, I., Connors, J., Gascoyne, R., Horsman, D., Jones, S. J., and Marra, M. a. (2009). Circos: An information aesthetic for comparative genomics. Genome Research, 19(9):1639-1645.

Kühlbrandt, W. (2014). The Resolution Revolution. Science, 343(6178):1443-1444.

Kulemzina, I., Schumacher, M. R., Verma, V., Reiter, J., Metzler, J., Failla, A. V., Lanz, C., Sreedharan, V. T., Rätsch, G., and Ivanov, D. (2012). Cohesin Rings Devoid of Scc3 and Pds5 Maintain Their Stable Association with the DNA. PLoS Genetics, 8(8).

Kurze, A., Michie, K. a., Dixon, S. E., Mishra, A., Itoh, T., Khalid, S., Strmecki, L., Shirahige, K., Haering, C. H., Löwe, J., and Nasmyth, K. (2011). A positively charged channel within the Smc1/Smc3 hinge required for sister chromatid cohesion. EMBO Journal, 30(2):364-378.

Ladurner, R., Bhaskara, V., Huis in 't Veld, P. J., Davidson, I. F., Kreidl, E., Petzold, G., and Peters, J.-M. (2014). Cohesin's ATPase Activity Couples Cohesin Loading onto DNA with Smc3 Acetylation. Current Biology, 24(19):2228-2237.

Lake, J. A. (1976). Ribosome structure determined by electron microscopy of Escherichia coli small subunits, large subunits and monomeric ribosomes. Journal of molecular biology, 105(1):131-139.

Lander, G. C., Estrin, E., Matyskiela, M. E., Bashore, C., Nogales, E., and Martin, A. (2012). Complete subunit architecture of the proteasome regulatory particle. Nature, 482(7384):186-191.

Langlois, R., Pallesen, J., and Frank, J. (2011). Reference-free particle selection enhanced with semi-supervised machine learning for cryo-electron microscopy. Journal of Structural Biology, 175(3):353-361.

Larionov, V. L., Karpova, T. S., Kouprina, N. Y., and Jouravleva, G. A. (1985). A mutant of Saccharomyces cerevisiae with impaired maintenance of centromeric plasmids. Current genetics, 10(1):15-20.

Lasker, K., Forster, F., Bohn, S., Walzthoeni, T., Villa, E., Unverdorben, P., Beck, F., Aebersold, R., Sali, a., and Baumeister, W. (2012a). Inaugural Article: Molecular architecture of the $26 \mathrm{~S}$ proteasome holocomplex determined by an integrative approach. Proceedings of the National Academy of Sciences, 109(5):1380-1387. 
Lasker, K., Forster, F., Bohn, S., Walzthoeni, T., Villa, E., Unverdorben, P., Beck, F., Aebersold, R., Sali, A., and Baumeister, W. (2012b). Molecular architecture of the 26S proteasome holocomplex determined by an integrative approach. Proceedings of the National Academy of Sciences of the United States of America, 109(5):1380-1387.

Leffers, H., Egebjerg, J., Andersen, A., Christensen, T., and Garrett, R. A. (1988). Domain VI of Escherichia coli $23 \mathrm{~S}$ ribosomal RNA. Structure, assembly and function. Journal of molecular biology, 204(3):507-522.

Leitner, A., Joachimiak, L. A., Bracher, A., Monkemeyer, L., Walzthoeni, T., Chen, B., Pechmann, S., Holmes, S., Cong, Y., Ma, B., Ludtke, S., Chiu, W., Hartl, F. U., Aebersold, R., and Frydman, J. (2012). The molecular architecture of the eukaryotic chaperonin TRiC/CCT. Structure (London, England: 1993), 20(5):814-825.

Lentzen, M. (2008). Contrast transfer and resolution limits for sub-angstrom highresolution transmission electron microscopy. Microscopy and microanalysis : the official journal of Microscopy Society of America, Microbeam Analysis Society, Microscopical Society of Canada, 14(1):16-26.

Leschziner, A. E. and Nogales, E. (2006). The orthogonal tilt reconstruction method: an approach to generating single-class volumes with no missing cone for ab initio reconstruction of asymmetric particles. Journal of structural biology, 153(3):284-299.

Lindahl, E., Azuara, C., Koehl, P., and Delarue, M. (2006). NOMAD-Ref: Visualization, deformation and refinement of macromolecular structures based on all-atom normal mode analysis. Nucleic Acids Research, 34(WEB. SERV. ISS.):52-56.

Liu, H., Rankin, S., and Yu, H. (2013). Phosphorylation-enabled binding of SGO1-PP2A to cohesin protects sororin and centromeric cohesion during mitosis. Nature cell biology, 15(1):40-9.

Liu, J. and Krantz, I. D. (2008). Cohesin and human disease. Annual review of genomics and human genetics, 9:303-320.

Losada, A., Hirano, M., and Hirano, T. (1998). Identification of Xenopus SMC protein complexes required for sister chromatid cohesion. Genes $\&$ development, 12(13):19861997.

Losada, A., Yokochi, T., and Hirano, T. (2005). Functional contribution of Pds5 to cohesin-mediated cohesion in human cells and Xenopus egg extracts. Journal of cell science, 118(Pt 10):2133-2141.

Losada, A., Yokochi, T., Kobayashi, R., and Hirano, T. (2000). Identification and characterization of SA/Scc3p subunits in the Xenopus and human cohesin complexes. The Journal of cell biology, 150(3):405-416. 
Lottspeich, F. and Engels, J. W. (2012). Bioanalytik. Spektrum, Heidelberg.

Lowe, J., Cordell, S. C., and van den Ent, F. (2001). Crystal structure of the SMC head domain: an ABC ATPase with 900 residues antiparallel coiled-coil inserted. Journal of molecular biology, 306(1):25-35.

Lupas, A. (1996). Prediction and analysis of coiled-coil structures. Methods in enzymology, 266:513-525.

Lupas, A., Van Dyke, M., and Stock, J. (1991). Predicting coiled coils from protein sequences. Science, 252(5009):1162-1164.

M, S. and H, S. (1980). Eine verbesserte Drehkorrelationsmethode für die Strukturbestimmung biologischer Makromoleküle durch Mittelung elektronenmikroskopischer Bilder. Hoppe-Seyler's Z. Physiolg. Chemie.

Maier, T., Leibundgut, M., and Ban, N. (2008). The crystal structure of a mammalian fatty acid synthase. Science, 321(5894):1315-1322.

Malumbres, M. and Barbacid, M. (2009). Cell cycle, CDKs and cancer: a changing paradigm. Nature reviews. Cancer, 9(3):153-166.

Maus, C. E., Plikaytis, B. B., and Shinnick, T. M. (2005). Molecular analysis of crossresistance to capreomycin, kanamycin, amikacin, and viomycin in Mycobacterium tuberculosis. Antimicrobial agents and chemotherapy, 49(8):3192-3197.

McCammon, J. A., Gelin, B. R., and Karplus, M. (1977). Dynamics of folded proteins. Nature, 267(5612):585-590.

McDonnell, A. V., Jiang, T., Keating, A. E., and Berger, B. (2006). Paircoil2: improved prediction of coiled coils from sequence. Bioinformatics, 22(3):356-358.

McGarry, K. G., Walker, S. E., Wang, H., and Fredrick, K. (2005). Destabilization of the $\mathrm{P}$ site codon-anticodon helix results from movement of tRNA into the $\mathrm{P} / \mathrm{E}$ hybrid state within the ribosome. Molecular Cell, 20(4):613-622.

Melby, T. E., Ciampaglio, C. N., Briscoe, G., and Erickson, H. P. (1998). The symmetrical structure of structural maintenance of chromosomes (SMC) and MukB proteins: long, antiparallel coiled coils, folded at a flexible hinge. The Journal of cell biology, 142(6):15951604.

Méndez, J. and Stillman, B. (2000). Chromatin Association of Human Origin Recognition Complex, Cdc6, and Minichromosome Maintenance Proteins during the Cell Cycle: Assembly of Prereplication Complexes in Late Mitosis. Molecular and Cellular Biology, 20(22):8602-8612.

Meyer, J. (1885). Meyers Konversations-Lexikon 4. Band. Brockhaus, Leipzig. 
Michaelis, C., Ciosk, R., and Nasmyth, K. (1997). Cohesins: Chromosomal proteins that prevent premature separation of sister chromatids. Cell, 91(1):35-45.

Mikolajka, A., Liu, H., Chen, Y., Starosta, A. L., Márquez, V., Ivanova, M., Cooperman, B. S., and Wilson, D. N. (2011). Differential effects of thiopeptide and orthosomycin antibiotics on translational GTPases. Chemistry \& biology, 18(5):589-600.

Moazed, D. and Noller, H. F. (1987). Chloramphenicol, erythromycin, carbomycin and vernamycin B protect overlapping sites in the peptidyl transferase region of $23 \mathrm{~S}$ ribosomal RNA. Biochimie, 69(8):879-884.

Moazed, D. and Noller, H. F. (1989a). Interaction of tRNA with $23 \mathrm{~S}$ rRNA in the ribosomal A, P, and E sites. Cell, 57(4):585-597.

Moazed, D. and Noller, H. F. (1989b). Intermediate states in the movement of transfer RNA in the ribosome. Nature, 342(6246):142-148.

Möckel, C., Lammens, K., Schele, A., and Hopfner, K. P. (2012). ATP driven structural changes of the bacterial Mre11:Rad50 catalytic head complex. Nucleic Acids Research, 40(2):914-927.

Monecke, T., Haselbach, D., Voß, B., Russek, A., Neumann, P., Thomson, E., and Hurt, E. (2012). Structural basis for cooperativity of CRM1 export complex formation. Pnas, $110(3): 960-965$.

Mueller, M., Jenni, S., and Ban, N. (2007). Strategies for crystallization and structure determination of very large macromolecular assemblies. Current Opinion in Structural Biology, 17(5):572-579.

Munro, J. B., Altman, R. B., O'Connor, N., and Blanchard, S. C. (2015). Identification of Two Distinct Hybrid State Intermediates on the Ribosome. Molecular Cell, 25(4):505517.

Nagayama, K. (2011). Another 60 years in electron microscopy: Development of phaseplate electron microscopy and biological applications. Journal of Electron Microscopy, 60(SUPPL. 1):43-62.

Nagayama, K. and Danev, R. (2009). Phase-plate electron microscopy: a novel imaging tool to reveal close-to-life nano-structures. Biophysical Reviews, 1(1):37-42.

Nasmyth, K. (2005). How might cohesin hold sister chromatids together? Philosophical transactions of the Royal Society of London. Series B, Biological sciences, 360(1455):483496.

Nasmyth, K. (2011). Cohesin: a catenase with separate entry and exit gates? Nature cell biology, 13(10):1170-7. 
Nasmyth, K. and Haering, C. H. (2005). The structure and function of SMC and kleisin complexes. Annual review of biochemistry, 74:595-648.

Niki, H., Imamura, R., Kitaoka, M., Yamanaka, K., Ogura, T., and Hiraga, S. (1992). E.coli MukB protein involved in chromosome partition forms a homodimer with a rod-and-hinge structure having DNA binding and ATP/GTP binding activities. EMBO Journal, 11(13):5101-5109.

Niki, H., Jaffe, A., Imamura, R., Ogura, T., and Hiraga, S. (1991). The new gene mukB codes for a $177 \mathrm{kd}$ protein with coiled-coil domains involved in chromosome partitioning of E. coli. EMBO Journal, 10(1):183-193.

Nishiyama, T., Ladurner, R., Schmitz, J., Kreidl, E., Schleiffer, A., Bhaskara, V., Bando, M., Shirahige, K., Hyman, A. A., Mechtler, K., and Peters, J.-M. (2010). Sororin mediates sister chromatid cohesion by antagonizing Wapl. Cell, 143(5):737-749.

Nogales-Cadenas, R., Jonic, S., Tama, F., Arteni, a. a., Tabas-Madrid, D., Vázquez, M., Pascual-Montano, a., and Sorzano, C. O. (2013). 3DEM Loupe: Analysis of macromolecular dynamics using structures from electron microscopy. Nucleic acids research, 41(Web Server issue):363-367.

Ogle, J. M. and Ramakrishnan, V. (2005). Structural insights into translational fidelity. Annual review of biochemistry, 74:129-177.

Orlova, E. V. and Saibil, H. R. (2011). Structural analysis of macromolecular assemblies by electron microscopy. Chemical reviews, 111(12):7710-48.

Ouyang, Z., Zheng, G., Song, J., Borek, D. M., Otwinowski, Z., Brautigam, C. a., Tomchick, D. R., Rankin, S., and Yu, H. (2013). Structure of the human cohesin inhibitor Wapl. Proceedings of the National Academy of Sciences of the United States of America, 110(28):11355-11360.

Pai, R. D., Zhang, W., Schuwirth, B. S., Hirokawa, G., Kaji, H., Kaji, A., and Cate, J. H. D. (2008). Structural Insights into Ribosome Recycling Factor Interactions with the 70S Ribosome. Journal of Molecular Biology, 376(5):1334-1347.

Palade, G. E. (1955). A small particulate component of the cytoplasm. The Journal of Cell Biology, 1(1):59-68.

Pan, D., Kirillov, S. V., and Cooperman, B. S. (2007). Kinetically competent intermediates in the translocation step of protein synthesis. Molecular cell, 25(4):519-529.

Panizza, S., Tanaka, T., Hochwagen, A., Eisenhaber, F., and Nasmyth, K. (2000). Pds5 cooperates with cohesin in maintaining sister chromatid cohesion. Current biology : $C B$, 10(24):1557-1564. 
Paulson, J. F. and Laemmli, U. K. (1977). Chromosomes of Histone-Depleted Metaphase. Cell, 12(November):817-828.

PDB Statistics (2015).

Penczek, P. A. (2010). Resolution measures in molecular electron microscopy. Methods in enzymology, 482:73-100.

Penczek, P. A., Grassucci, R. A., and Frank, J. (1994). The ribosome at improved resolution: new techniques for merging and orientation refinement in $3 \mathrm{D}$ cryo-electron microscopy of biological particles. Ultramicroscopy, 53(3):251-270.

Penczek, P. A., Yang, C., Frank, J., and Spahn, C. M. T. (2006). Estimation of variance in single-particle reconstruction using the bootstrap technique. Journal of Structural Biology, 154:168-183.

Peske, F., Savelsbergh, A., Katunin, V. I., Rodnina, M. V., and Wintermeyer, W. (2004). Conformational Changes of the Small Ribosomal Subunit During Elongation Factor G-dependent tRNA-mRNA Translocation. Journal of Molecular Biology, 343(5):11831194.

Peters, J.-M. (2002). The Anaphase-Promoting Complex. Molecular Cell, 9(5):931-943.

Peters, J.-M. and Nishiyama, T. (2012). Sister Chromatid Cohesion. Cold Spring Harbor Perspectives in Biology, 4(11):a011130-a011130.

Peters, J.-M., Tedeschi, A., and Schmitz, J. (2008). The cohesin complex and its roles in chromosome biology. Genes \& development, 22(22):3089-114.

Philipse, a. P. (2011). Notes on Brownian Motion.

Pintilie, G. D., Zhang, J., Goddard, T. D., Chiu, W., and Gossard, D. C. (2010). Quantitative analysis of cryo-EM density map segmentation by watershed and scale-space filtering, and fitting of structures by alignment to regions. Journal of Structural Biology, 170(3):427-438.

Quirk, T. (2006). There's plenty of room at the bottom. Australasian Biotechnology, $16(3): 36$.

Radermacher, M., Wagenknecht, T., Verschoor, A., and Frank, J. (1987). Threedimensional reconstruction from a single-exposure, random conical tilt series applied to the 50S ribosomal subunit of Escherichia coli. Journal of microscopy, 146(Pt 2):113-136.

Rasmussen, B. F., Stock, a. M., Ringe, D., and Petsko, G. a. (1992). Crystalline ribonuclease A loses function below the dynamical transition at 220 K. Nature, 357(6377):423-424. 
Riedel, C. G., Katis, V. L., Katou, Y., Mori, S., Itoh, T., Helmhart, W., Gálová, M., Petronczki, M., Gregan, J., Cetin, B., Mudrak, I., Ogris, E., Mechtler, K., Pelletier, L., Buchholz, F., Shirahige, K., and Nasmyth, K. (2006). Protein phosphatase 2A protects centromeric sister chromatid cohesion during meiosis I. Nature, 441(7089):53-61.

Rodnina, M. V., Beringer, M., and Wintermeyer, W. (2007). How ribosomes make peptide bonds. Trends in Biochemical Sciences, 32(1):20-26.

Rodnina, M. V., Savelsbergh, A., Katunin, V. I., and Wintermeyer, W. (1997). Hydrolysis of GTP by elongation factor G drives tRNA movement on the ribosome. Nature, 385(6611):37-41.

Rodnina, Marina V., Wintermeyer, Wolfgang, Green, R. (2011). Ribosomes Structure, Function, and Dynamics. Springer, Wien.

Rosenthal, P. B. and Henderson, R. (2003). Optimal Determination of Particle Orientation, Absolute Hand, and Contrast Loss in Single-particle Electron Cryomicroscopy. Journal of Molecular Biology, 333(4):721-745.

Rost, B., Yachdav, G., and Liu, J. (2004). The PredictProtein server. Nucleic acids research, 32(Web Server issue):W321-6.

Rowland, B. D., Roig, M. B., Nishino, T., Kurze, A., Uluocak, P., Mishra, A., Beckouet, F., Underwood, P., Metson, J., Imre, R., Mechtler, K., Katis, V. L., and Nasmyth, K. (2009). Building sister chromatid cohesion: smc3 acetylation counteracts an antiestablishment activity. Molecular cell, 33(6):763-774.

Saitoh, N. (1994). ScII: an abundant chromosome scaffold protein is a member of a family of putative ATPases with an unusual predicted tertiary structure. The Journal of Cell Biology, 127(2):303-318.

Sander, B., Golas, M. M., and Stark, H. (2003). Corrim-based alignment for improved speed in single-particle image processing. Journal of Structural Biology, 143(3):219-228.

Sander, B., Golas, M. M., and Stark, H. (2005). Advantages of CCD detectors for de novo three-dimensional structure determination in single-particle electron microscopy. Journal of Structural Biology, 151(1):92-105.

Saxton, W. O. and Baumeister, W. (1982). The correlation averaging of a regularly arranged bacterial cell envelope protein. Journal of microscopy, 127(Pt 2):127-138.

Scheres, S. H. W. (2012a). A Bayesian view on cryo-EM structure determination. Journal of molecular biology, 415(2):406-18.

Scheres, S. H. W. (2012b). RELION: implementation of a Bayesian approach to cryo-EM structure determination. Journal of structural biology, 180(3):519-30. 
Scheres, S. H. W. (2014). Beam-induced motion correction for sub-megadalton cryo-EM particles. eLife, 3:e03678.

Scheres, S. H. W. and Chen, S. (2012). Prevention of overfitting in cryo-EM structure determination. Nature Methods, 9(9):853-854.

Scheres, S. H. W., Valle, M., Nuñez, R., Sorzano, C. O. S., Marabini, R., Herman, G. T., and Carazo, J. M. (2005). Maximum-likelihood multi-reference refinement for electron microscopy images. Journal of Molecular Biology, 348(1):139-149.

Schluenzen, F., Tocilj, A., Zarivach, R., Harms, J., Gluehmann, M., Janell, D., Bashan, A., Bartels, H., Agmon, I., Yonath, A., and Ribosomal, M.-p.-r. U. (2000). Small Ribosomal Subunit Resolution at 3.3 A. Structure, 102:615-623.

Schmeing, T. M. and Ramakrishnan, V. (2009). What recent ribosome structures have revealed about the mechanism of translation. Nature, 461(7268):1234-1242.

Schmitz, J., Watrin, E., Lenart, P., Mechtler, K., and Peters, J.-M. (2007). Sororin is required for stable binding of cohesin to chromatin and for sister chromatid cohesion in interphase. Current biology : CB, 17(7):630-636.

Schuwirth, B. S., Borovinskaya, M. A., Hau, C. W., Zhang, W., Vila-Sanjurjo, A., Holton, J. M., and Cate, J. H. D. (2005). Structures of the Bacterial Ribosome at 3.5 AResolution. Science, 310(5749):827-834.

Semenkov, Y. P., Rodnina, M. V., and Wintermeyer, W. (2000). Energetic contribution of tRNA hybrid state formation to translocation catalysis on the ribosome. Nat Struct Mol Biol, 7(11):1027-1031.

Serdyuk, I. N., Zaccai, N. R., and Zaccai, J. (2007). Methods in Moelcular Biophysics. Cambridge University Press, Cambridge.

Sharon, M. (2010). How far can we go with structural mass spectrometry of protein complexes? Journal of the American Society for Mass Spectrometry, 21(4):487-500.

Shintomi, K. and Hirano, T. (2009). Releasing cohesin from chromosome arms in early mitosis: opposing actions of Wapl-Pds5 and Sgo1. Genes $\&$ development, 23(18):22242236 .

Shoji, S., Walker, S. E., and Fredrick, K. (2006). Reverse translocation of tRNA in the ribosome. Molecular cell, 24(6):931-942.

Singer, A., Coifman, R. R., Sigworth, F. J., Chester, D. W., and Shkolnisky, Y. (2010). Detecting consistent common lines in cryo-EM by voting. Journal of Structural Biology, 169(3):312-322. 
Skjaerven, L., Hollup, S. M., and Reuter, N. (2009). Normal mode analysis for proteins. Journal of Molecular Structure: THEOCHEM, 898(1-3):42-48.

Soding, J. (2005). Protein homology detection by HMM-HMM comparison. Bioinformatics, 21(7):951-960.

Soh, Y.-M., Bürmann, F., Shin, H.-C., Oda, T., Jin, K. S., Toseland, C. P., Kim, C., Lee, H., Kim, S. J., Kong, M.-S., Durand-Diebold, M.-L., Kim, Y.-G., Kim, H. M., Lee, N. K., Sato, M., Oh, B.-H., and Gruber, S. (2015). Molecular Basis for SMC Rod Formation and Its Dissolution upon DNA Binding. Molecular Cell, 57(2):290-303.

Stanley, R. E., Blaha, G., Grodzicki, R. L., Strickler, M. D., and Steitz, T. a. (2010). The structures of the anti-tuberculosis antibiotics viomycin and capreomycin bound to the 70S ribosome. Nature structural \& molecular biology, 17(3):289-93.

Stark, H., Rodnina, M. V., Rinke-Appel, J., Brimacombe, R., Wintermeyer, W., and van Heel, M. (1997). Visualization of elongation factor $\mathrm{Tu}$ on the Escherichia coli ribosome. Nature, 389(6649):403-6.

Stark, H., Rodnina, M. V., Wieden, H. J., van Heel, M., and Wintermeyer, W. (2000). Large-scale movement of elongation factor $\mathrm{G}$ and extensive conformational change of the ribosome during translocation. Cell, 100(3):301-309.

Stark, H., Rodnina, M. V., Wieden, H.-J., Zemlin, F., Wintermeyer, W., and van Heel, M. (2002). Ribosome interactions of aminoacyl-tRNA and elongation factor Tu in the codon-recognition complex. Nature structural biology, 9(11):849-854.

Stark, H., Zemlin, F., and Boettcher, C. (1996). Electron radiation damage to protein crystals of bacteriorhodopsin at different temperatures. Ultramicroscopy, 63:75-80.

Steitz, T. a. (2008). A structural understanding of the dynamic ribosome machine. Nature reviews. Molecular cell biology, 9(3):242-253.

Stewart, A. and Grigorieff, N. (2004). Noise bias in the refinement of structures derived from single particles. Ultramicroscopy, 102(1):67-84.

Strom, L., Karlsson, C., Lindroos, H. B., Wedahl, S., Katou, Y., Shirahige, K., and Sjogren, C. (2007). Postreplicative formation of cohesion is required for repair and induced by a single DNA break. Science, 317(5835):242-245.

Sumara, I., Vorlaufer, E., Gieffers, C., Peters, B. H., and Peters, J.-M. (2000). Characterization of Vertebrate Cohesin Complexes and Their Regulation in Prophase. The Journal of Cell Biology, 151(4):749-762. 
Sumara, I., Vorlaufer, E., Stukenberg, P. T., Kelm, O., Redemann, N., Nigg, E. A., and Peters, J.-M. (2002). The dissociation of cohesin from chromosomes in prophase is regulated by Polo-like kinase. Molecular cell, 9(3):515-525.

Sykes, M. T. and Williamson, J. R. (2009). A complex assembly landscape for the 30S ribosomal subunit. Annual review of biophysics, 38:197-215.

Tachibana-Konwalski, K., Godwin, J., van der Weyden, L., Champion, L., Kudo, N. R., Adams, D. J., and Nasmyth, K. (2010). Rec8-containing cohesin maintains bivalents without turnover during the growing phase of mouse oocytes. Genes 85 development, 24(22):2505-2516.

Takahashi, T. S., Basu, A., Bermudez, V., Hurwitz, J., and Walter, J. C. (2008). Cdc7-Drf1 kinase links chromosome cohesion to the initiation of DNA replication in Xenopus egg extracts. Genes \& Development, 22(14):1894-1905.

Takahashi, T. S., Yiu, P., Chou, M. F., Gygi, S., and Walter, J. C. (2004). Recruitment of Xenopus Scc2 and cohesin to chromatin requires the pre-replication complex. Nature cell biology, 6(10):991-996.

Thomas, M. G., Chan, Y. A., and Ozanick, S. G. (2003). Deciphering Tuberactinomycin Biosynthesis: Isolation, Sequencing, and Annotation of the Viomycin Biosynthetic Gene Cluster. Antimicrobial Agents and Chemotherapy, 47(9):2823-2830.

Toth, A., Ciosk, R., Uhlmann, F., Galova, M., Schleiffer, A., and Nasmyth, K. (1999). Yeast cohesin complex requires a conserved protein, Eco1p(Ctf7), to establish cohesion between sister chromatids during DNA replication. Genes $\&$ development, 13(3):320-333.

Uhlmann, F., Lottspeich, F., and Nasmyth, K. (1999). Sister-chromatid separation at anaphase onset is promoted by cleavage of the cohesin subunit Scc1. Nature, 400(6739):37-42.

Uhlmann, F., Wernic, D., Poupart, M. A., Koonin, E. V., and Nasmyth, K. (2000). Cleavage of cohesin by the CD clan protease separin triggers anaphase in yeast. Cell, 103(3):375-386.

Unal, E., Heidinger-Pauli, J. M., and Koshland, D. (2007). DNA double-strand breaks trigger genome-wide sister-chromatid cohesion through Eco1 (Ctf7). Science, 317(5835):245248.

Van Heel, M. (1987). Angular reconstitution: a posteriori assignment of projection directions for 3D reconstruction. Ultramicroscopy, 21(2):111-123. 
van Heel, M. (2013). Finding trimeric HIV-1 envelope glycoproteins in random noise. Proceedings of the National Academy of Sciences of the United States of America, 110(45):E4175-E4177.

van Heel, M., Harauz, G., Orlova, E. V., Schmidt, R., and Schatz, M. (1996). A new generation of the IMAGIC image processing system. Journal of structural biology, 116(1):17-24.

van Heel, M., Portugal, R., and Schatz, M. (2009). Multivariate Statistical Analysis in Single Particle (Cryo) Electron Microscopy. An electronic text book: Electron microscopy in Life Science, pages 1-47.

van Heel, M. and Stöffler-Meilicke, M. (1985). Characteristic views of E. coli and B. stearothermophilus $30 \mathrm{~S}$ ribosomal subunits in the electron microscope. EMBO Journal, 4(9):2389-2395.

Voorhees, R. M. and Ramakrishnan, V. (2013). Structural basis of the translational elongation cycle. Annual review of biochemistry, 82:203-36.

Voorhees, R. M., Weixlbaumer, A., Loakes, D., Kelley, A. C., and Ramakrishnan, V. (2009). Insights into substrate stabilization from snapshots of the peptidyl transferase center of the intact 70S ribosome. Nature structural \& molecular biology, 16(5):528-533.

Voss, N. R., Yoshioka, C. K., Radermacher, M., Potter, C. S., and Carragher, B. (2009). DoG Picker and TiltPicker: software tools to facilitate particle selection in single particle electron microscopy. Journal of structural biology, 166(2):205-213.

Waizenegger, I. C., Hauf, S., Meinke, A., and Peters, J.-M. (2000). Two Distinct Pathways Remove Mammalian Cohesin from Chromosome Arms in Prophase and from Centromeres in Anaphase. Cell, 103(3):399-410.

Walker, S. E., Shoji, S., Pan, D., Cooperman, B. S., and Fredrick, K. (2008). Role of hybrid tRNA-binding states in ribosomal translocation. Proceedings of the National Academy of Sciences, 105(27):9192-9197.

Walzthoeni, T., Leitner, A., Stengel, F., and Aebersold, R. (2013). Mass spectrometry supported determination of protein complex structure. Current Opinion in Structural Biology, 23(2):252-260.

Watanabe, Y. and Nurse, P. (1999). Cohesin Rec8 is required for reductional chromosome segregation at meiosis. Nature, 400(6743):461-464.

Weitzer, S., Lehane, C., and Uhlmann, F. (2003). A Model for ATP Hydrolysis-Dependent Binding of Cohesin to DNA. Current Biology, 13(22):1930-1940. 
Williams, D. B. and Carter, C. B. (2009). Transmission electron microscopy., volume 2. Springer, New York.

Wilson, D. N. (2009). The A-Z of bacterial translation inhibitors. Critical reviews in biochemistry and molecular biology, 44(6):393-433.

Wimberly, B. T., Brodersen, D. E., Clemons, W. M., Morgan-Warren, R. J., Carter, A. P., Vonrhein, C., Hartsch, T., and Ramakrishnan, V. (2000). Structure of the 30S ribosomal subunit. Nature, 407(6802):327-339.

Wohlgemuth, I., Pohl, C., and Rodnina, M. V. (2010). Optimization of speed and accuracy of decoding in translation. EMBO Journal, 29(21):3701-3709.

Yamada, T. and Bierhaus, K. H. (1978). Viomycin favours the formation of 70 S ribosome couples. Molecular \& general genetics : $M G G, 161(3): 261-265$.

Yin, X., O'Hare, T., Gould, S. J., and Zabriskie, T. M. (2003). Identification and cloning of genes encoding viomycin biosynthesis from Streptomyces vinaceus and evidence for involvement of a rare oxygenase. Gene, 312:215-224.

Yonath, a., Leonard, K. R., and Wittmann, H. G. (1987). A tunnel in the large ribosomal subunit revealed by three-dimensional image reconstruction. Science, 236(4803):813-816.

Yusupov, M. M., Yusupova, G. Z., Baucom, A., Lieberman, K., Earnest, T. N., Cate, J. H., and Noller, H. F. (2001). Crystal structure of the ribosome at 5.5 A resolution. Science, 292(5518):883-896.

Zemlin, F., Weiss, K., and Schiske, P. (1978). Coma-free alignment of high resolution electron microscopes with the aid of optical diffractograms. Ultramicroscopy, 3(1):49-60.

Zhang, B. and Cech, T. R. (1997). Peptide bond formation by in vitro selected ribozymes. Nature, 390(6655):96-100.

Zhang, N., Panigrahi, A. K., Mao, Q., and Pati, D. (2011). Interaction of Sororin protein with polo-like kinase 1 mediates resolution of chromosomal arm cohesion. The Journal of biological chemistry, 286(48):41826-41837.

Zhao, J., Brubaker, M. A., and Rubinstein, J. L. (2013). TMaCS: a hybrid template matching and classification system for partially-automated particle selection. Journal of structural biology, 181(3):234-242.

Zhu, Y., Carragher, B., Glaeser, R. M., Fellmann, D., Bajaj, C., Bern, M., Mouche, F., de Haas, F., Hall, R. J., Kriegman, D. J., Ludtke, S. J., Mallick, S. P., Penczek, P. A., Roseman, A. M., Sigworth, F. J., Volkmann, N., and Potter, C. S. (2004). Automatic particle selection: results of a comparative study. Journal of structural biology, 145(1-2):3-14. 
Zimmerman, S. B. and Minton, A. P. (1993). Marcomolecular Crowding: Biochemical, Biophysical, and Physiological Consequences. Annu. Rev. Biophys. Biomol. Struct., 22:27-65:27-65. 


\section{Publications of the Author}

1. M. Hons*, B. Niebel*, N. Karnowski, B. Weiche, M. Famulok (2012). Pan-Selective Aptamers for the Family of Small GTPases. ChemBioChem 13(10):1433-1437

2. M. Hons*,P. J. Huis in 't Veld*, J. Kaesler, P. Rombaut, A. Schleiffer, F. Herzog, H. Stark, J.-M. Peters (2015). Topology and structure of an engineered human cohesin complex bound to Pds5B. manuscript in submission

* Authors contributed equally. 



\title{
Curriculum Vitae
}

\author{
Michael Hons \\ born $18^{\text {th }}$ December 1984 in Aachen, Germany \\ Prinzenstraße 10-12, 37073 Göttingen \\ Phone +49 (0) 551 201-1315 \\ E-Mail mhons@mpibpc.mpg.de
}

Selected Workshops and Conferences

07/03/2016 - 07/03/2016 Visualizing Biological Data (VIZBI), EMBL, Heidelberg

09/11/2014 - 14/11/2014 A Workshop on Advanced Topics in EM Structure Determination, National Resource for Automated Molecular Microscopy (NRAMM), The Scripps Research Institute, La Jolla

26/08/2012 - 02/09/2012 Cryo-Electron Microscopy and 3D Image Processing, EMBO Practical Course, EMBL, Heidelberg

\author{
Internships \\ 02/2009-04/2009 \\ 08/2007 - 09/2007 \\ University education \\ 05/2015 - present \\ $03 / 2011-05 / 2015$
}

$10 / 2005-12 / 2010$
Bayer HealthCare Pharmaceuticals, Structural Biology / Lead Discovery (Dr. Ursula Egner), Berlin

Institute of Reconstructive Neurobiology, Stem Cell Engineering Group (Dr. Frank Edenhofer), Bonn 
